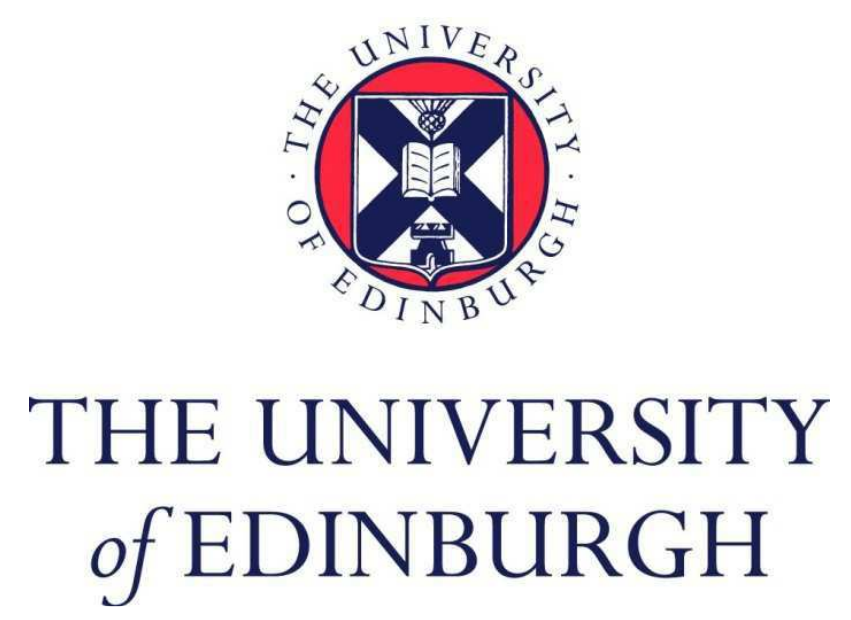

This thesis has been submitted in fulfilment of the requirements for a postgraduate degree (e.g. PhD, MPhil, DClinPsychol) at the University of Edinburgh. Please note the following terms and conditions of use:

This work is protected by copyright and other intellectual property rights, which are retained by the thesis author, unless otherwise stated.

A copy can be downloaded for personal non-commercial research or study, without prior permission or charge.

This thesis cannot be reproduced or quoted extensively from without first obtaining permission in writing from the author.

The content must not be changed in any way or sold commercially in any format or medium without the formal permission of the author.

When referring to this work, full bibliographic details including the author, title, awarding institution and date of the thesis must be given. 


\section{Polymeric Frustrated Lewis Pairs}

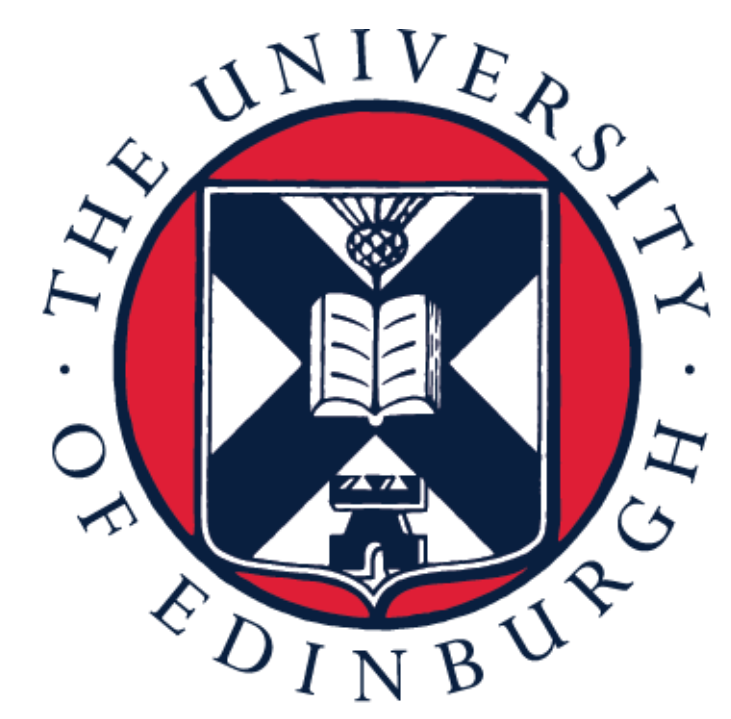

Meng Wang

A thesis submitted to the University of Edinburgh for the Degree of Doctor of Philosophy 2019 


\section{Abstract}

Frustrated Lewis Pair (FLP) chemistry is a significant and growing field since it offers a novel non-metal catalyst for hydrogenation and small molecule activation. Once it was discovered, different FLPs with varying reactivity towards small molecules have been extensively investigated. Its research has mainly focused on small moleculebased FLPs, however, especially in the aspect of hydrogenation reactions. In the field of polymer chemistry, several examples of conventional Lewis pair adduct containing polymers have been reported but there has yet been no exploration of FLPs incorporated into polymers up to the date of this project.

Dynamic crosslinked polymeric networks have attracted more attention in recent years as their shape can be post-modified after polymerisation due to their exchangeable crosslinks. This dynamic crosslinking also makes the material stimuli-responsive and provides self-healing properties.

This thesis introduces the synthesis of a polymeric network with combined features of frustrated Lewis pairs and dynamic crosslinking. New monomers containing Lewis acid or Lewis base centres were designed and synthesised successfully. For the pair 4styryl-diphenylborane and 4-styryl-diphenylphosphine, the two monomers were found to be able to bind together at high concentration in toluene so as to form a weak conventional Lewis pair (CLP) adduct. An FLP can be obtained when the phosphine monomer was replaced to its more hindered analogue, 4-styryl-dimesitylphosphine, which is reactive enough to form a complex with diethyl azodicarboxylate (DEAD), where the DEAD bridges the boron and phosphorous centres. The monomers obtained were copolymerised with styrene by RAFT polymerisations. It was also found to be possible to control both the molecular weight and the dispersity. The FLP polymers 
synthesised in this way were characterised by NMR spectroscopy and gel permission chromatography. The Lewis acidity of both the monomer and resultant polymer were tested using the Gutmann-Beckett Method, and a decrease in Lewis acidity was observed when the boron monomer was polymerised. The network was synthesised by addition of DEAD into the solution containing both Lewis acid and Lewis base polymers. A gel was quickly generated (in 10 seconds). The mechanical properties of the network formed were determined by rheology. The gel was responsive to heat, in that it would break and return to a polymer solution at high temperatures. The gel formed also shows the ability to self-heal with the assistance of a solvent after physical cracking.

The synthesis of the next generation of polymeric FLPs was also examined. A much more Lewis acidic boron monomer, (2,3,5,6-tetrafluorostyryl)bis(pentafluorophenyl)borane was synthesised. This boron monomer was paired with 4-styryl-dimesitylphosphine to form a reactive FLP that was able to activate small molecules, including dihydrogen molecules and carbon dioxide. The catalysis reactivity of the hydrogenation reactions of this FLP was also explored. The copolymers made from these reactions readily formed a supramolecular gel upon mixing, which also proved temperature responsive. These early-stage results proved that this new boron-monomer is capable of generating a novel stimuli-responsive smart polymer for carbon capture and hydrogenation catalysis. Except for the polymeric FLP, some early-stage research about polymeric CLP and novel synthetic methods for boron-monomers were also introduced and discussed. 


\section{Lay Summary}

Dynamic crosslinked polymer materials have received a lot of attention because they can be reshaped, reprocessed and self-healable. The reshaping and healing of this class of material is normally achieved by stimuli-triggered crosslinking exchanges. There are many dynamic bonds, either covalent or supramolecular, used as crosslinks in polymer networks, including carbon-carbon bonds based on reversible Diels-Alder or cycloaddition reactions, boronic ester or boroxine bonding, siloxane bonds, disulfide/thiol bonds, hydrogen bonds, ionic bonds, $\pi-\pi$ stacking, and Lewis pair complexation. However, use of frustrated Lewis pairs (FLPs) as the dynamic crosslinking of a polymer gel was unprecedented. This thesis investigates the synthesis of macromolecular FLPs as a macro-gelators for generating novel dynamic crosslinked polymer networks.

4-styryl-dimesitylphoshine and 4-styryl-diphenylborane were used to make copolymers with styrene. The resultant copolymers cannot form polymer gel upon mixing due to the steric hindrance around the boron and phosphorus. Gelation was only triggered when a small molecule diethyl azodicarboxylate was added, which linked up the Lewis acids and Lewis bases. Rheology study showed that the resultant poly(FLP) gel is supramolecularly crosslinked, temperature responsive and selfhealable.

Finally, a fully fluorinated boron containing monomer was synthesised. This monomer showed dramatically increased Lewis acidity, and was able to activate dihydrogen and carbon dioxide when paring with the phosphine monomer. This makes it ideal to be used in the next generation macromolecular FLPs for polymer network synthesis and catalysis. 


\section{Declaration}

I declare that this thesis was composed by myself, and all the work described here is of my own, unless I have acknowledged help from a named person or referenced a published work. This thesis has not been submitted, in whole or in part, for any other degree or professional qualification.

Meng Wang

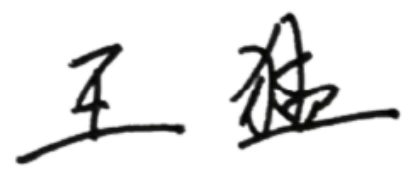

May 2019 


\section{Acknowledgments}

The research work involved in this thesis would not have been possible without the kind support of many people, which I deeply appreciate. First of all, I would like to express my sincere gratitude to my supervisor, Prof. Michael P. Shaver, not only for providing me with an opportunity to join the Green Materials Laboratory, but also for his patient supervision of the project and enthusiasm for chemistry. His expertise ensured the completion of my project, and his valuable advice has also helped me to become a more competent chemist. I would also like to thank the School of Chemistry at the University of Edinburgh for awarding me a Tercentenary Scholarship.

I would like to thank all the past and present group members, for their huge support for my research and for providing lots of valuable advice in the lab. Special thanks to: Stefan, who makes the research life of all the group members so colourful. Ben, for his knowledge that all of us benefit from. Jarret, Emily, Fern, Genny for teaching me and for their familiarity with the lab work. Panos and Rebecca, for their hard work in my mentoring. Utku, who also joined the same project, made outstanding contributions with his rheology skills. Mitch, for his patient help with this thesis. Yuechao, for his conscientiousness and sense of responsibility to the group. Thanks to all the members in the McKeown group, who are nice neighbours to work nearby. Also thanks to all the people from the Garden group for their kind help.

I would also like to show my appreciation to all the people in the College of Science and Engineering who helped me with my research. Dr Lorna Murray and Mr Juraj Bella for NMR spectroscopy. Dr Logan Mackay for training and assistance with the mass spectrometer. Mr Stuart Johnstone for accepting my unlimited amount of glassware orders. Special thanks to Dr Fabio Nudelman, for his support on TEM and 
SEM in my published work. Dr John Royer from the School of Physics, for his kind help with rheology measurements.

Finally, I wish to thank my friends and family for their support throughout my life. Yi Li and Tairan Wang, for their 15-year-friendship, which for sure will continue and lead to more stories among us. My parents, thank you both for understanding and for your support for all the life decisions I made since from I left home. You have brought so much to me, but what I have done for you is certainly far from enough. 


\section{Table of Contents}

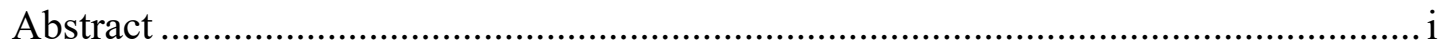

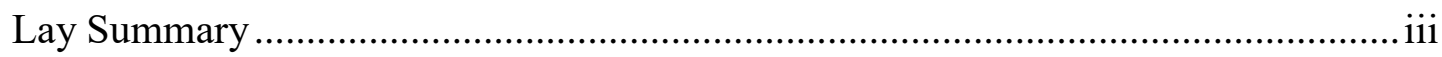

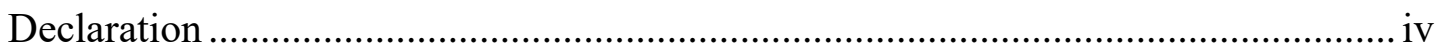

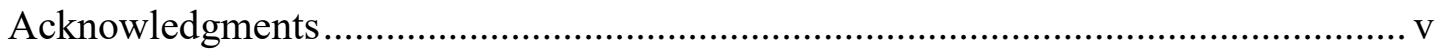

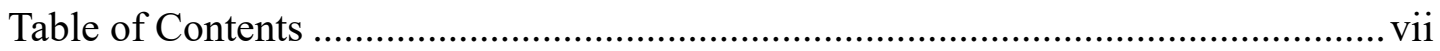

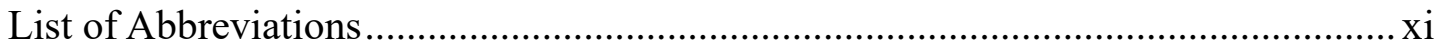

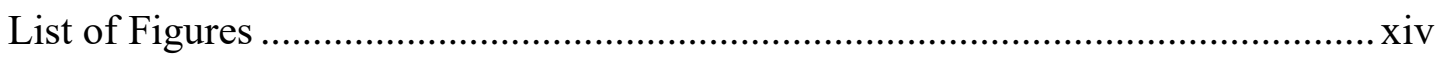

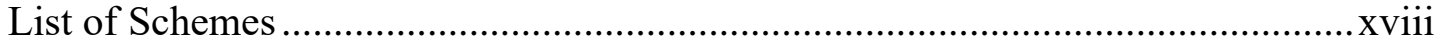

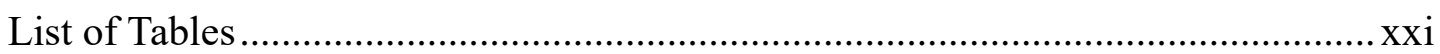

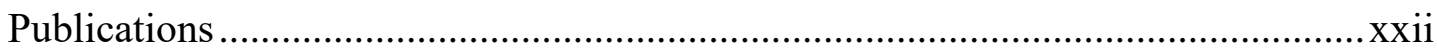

Chapter One. Frustrated Lewis Pairs and Dynamic Crosslinked Polymeric

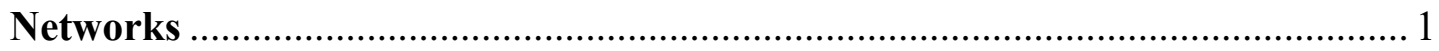

1.1 Introduction to Frustrated Lewis Pairs ....................................................... 1

1.1.1 Lewis Pair Adducts and Frustrated Lewis Pairs................................ 1

1.1.1.1 History of Acid/Bases Theory .............................................. 1

1.1.1.2 Discovery of Frustrated Lewis Pairs ...................................... 3

1.1.2 FLP reactivity and Lewis Basicity/Acidity ..................................... 5

1.1.2.1 Lewis Basicity Scales...................................................... 6

1.1.2.2 Lewis Acidity Scales ....................................................... 10

1.1.3 Applications of Frustrated Lewis Pairs ........................................... 12

1.1.3.1 Metal-free Hydrogenations ................................................... 13

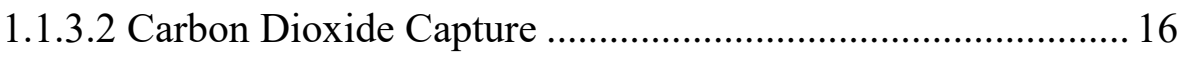

1.1.3.3 FLPs as Catalysts for Polymerisations ................................ 18 
1.2 Dynamic Crosslinked Polymeric Networks and Self-Healing Materials..... 20

1.2.1 Covalent Adaptable Networks .................................................... 20

1.2.2 Supramolecular Chemistry Based Polymeric Networks ................. 22

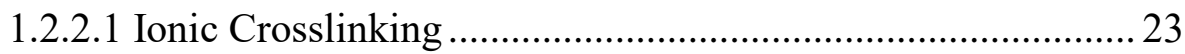

1.2.2.2 $\pi-\pi$ Stacking Interaction Crosslinking................................ 25

1.2.2.3 Hydrogen Bond Crosslinking............................................. 26

1.2.2.4 Van der Waals Forces based Crosslinking............................ 28

1.2.2.5 Conventional Lewis Pair Based Crosslinking ....................... 30

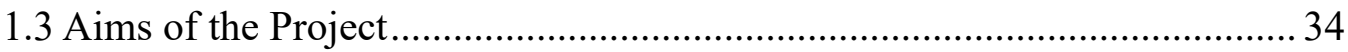

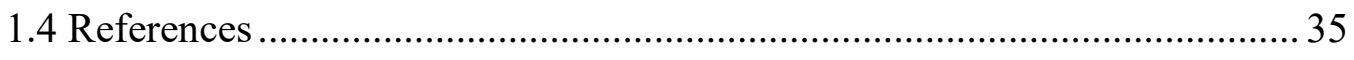

Chapter Two. Polymeric Frustrated Lewis Pairs from Boron and Phosphorous

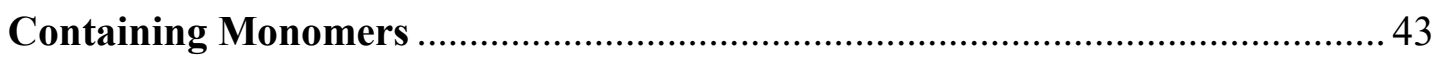

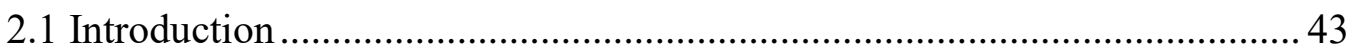

2.1.1 Organoboron Chemistry ......................................................... 43

2.1.2 Organophosphorus Chemistry .................................................. 46

2.2 Synthesis and Characterisation of the Phosphorus-/Boron-containing

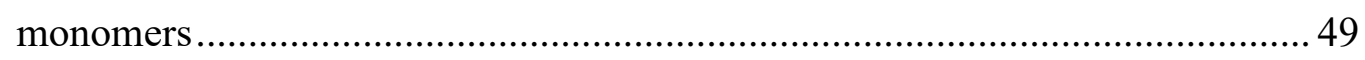

2.2.1 Lewis Basic Monomers................................................................ 49

2.2.1.1 Synthesis of 4-styryl-diphenylphosphine $\left(\mathbf{S t P P h}_{2}\right)$.............. 49

2.2.1.2 Synthesis of 4-Styryl-dimesitylphosphine (StPMes2) .......... 50

2.2.1.3 Determination of Lewis Basicity ........................................ 51

2.2.2 Lewis Acidic Monomers …............................................................... 53

2.2.2.1 Synthesis of 4-styryl-diphenylborane $\left(\mathbf{S t B P h}_{2}\right) \ldots \ldots \ldots \ldots \ldots \ldots . . . . . . . . . .53$

2.2.2.2 Determination of Lewis Acidity .......................................... 64

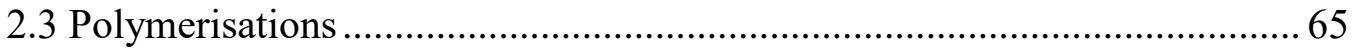

2.3.1 Polymerisation of Lewis Basic Monomers ...................................... 65

2.3.2 Polymerisation of Lewis Acidic Monomers..................................... 69

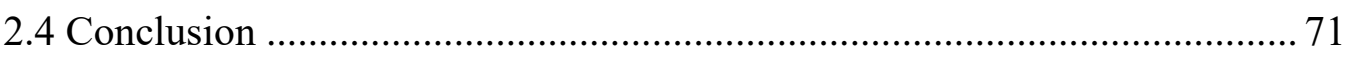


2.5 References

Chapter Three. Polymeric Frustrated Lewis Pairs as Network Macro-gelators 75

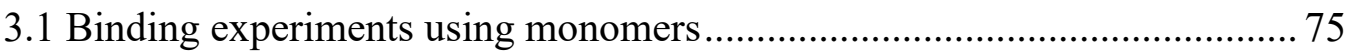

3.1.1 Formation of Lewis Pair Adduct between $\mathbf{S t P P h} 2$ and $\mathbf{S t B P h} 2$ as Control Experiment 75

3.1.2 Formation of Frustrated Lewis Pair using StPMes2 and StBPh2 and

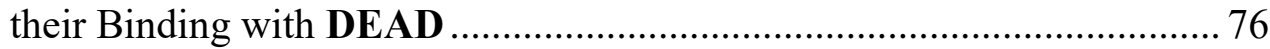

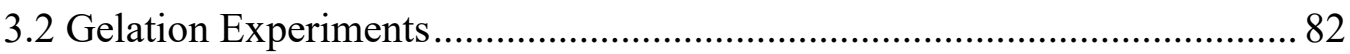

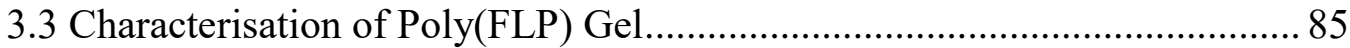

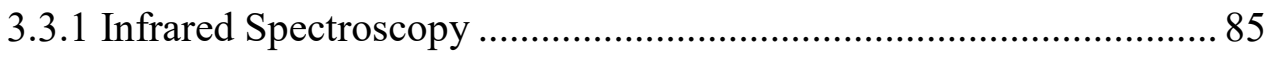

3.3.2 Swelling Ratio Measurement ............................................................ 86

3.3.3 Scanning Electron Microscopy …............................................... 87

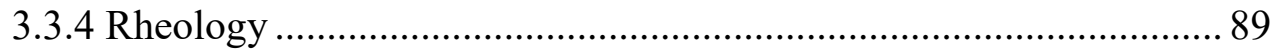

3.3.4.1 Dynamic Nature of Crosslinking ........................................ 90

3.3.4.2 Time-Temperature Superposition......................................... 91

3.3.4.3 Temperature Responsiveness ........................................... 93

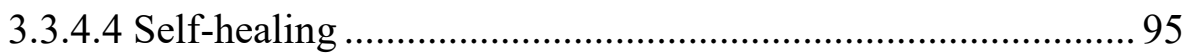

3.4 DEAD Triggered Poly(CLP) Gel............................................................. 98

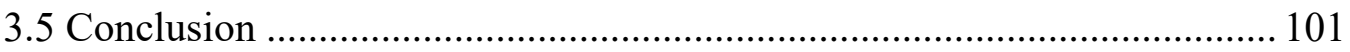

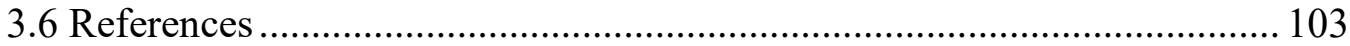

Chapter Four. Novel Boron-Containing Monomers for Next Generation Polymeric Frustrated Lewis Pairs ................................................................... 105

4.1 (2,3,5,6-tetrafluorostyryl)-bis-(pentafluorophenyl)borane $\left(\mathbf{S t F}_{\mathbf{4}}\right) \mathbf{B}\left(\mathbf{C}_{\mathbf{6}} \mathbf{F}_{5}\right)_{\mathbf{2}} 107$

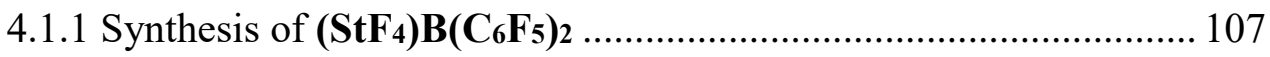

4.1.2 Binding test with StPMes2 ..................................................... 112

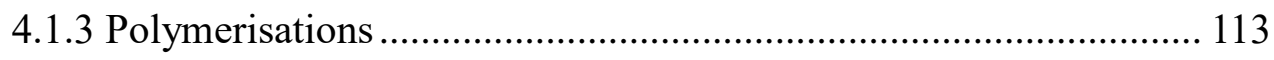

4.1.4 Gelation Experiment …........................................................... 114

4.1.5 Small Molecule Activation.......................................................... 117 
4.2 Other Boron-containing Monomers

4.2.1 Boron-functionalised Monomers by Hydroboration.

4.2.2 Expansion of Coordination-Recovery Strategy ............................ 123

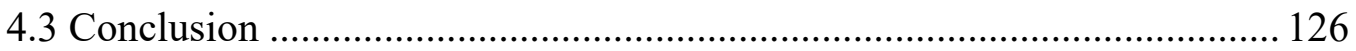

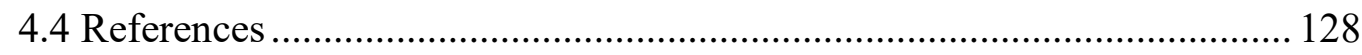

Chapter Five. Conclusions and Future Work................................................. 130

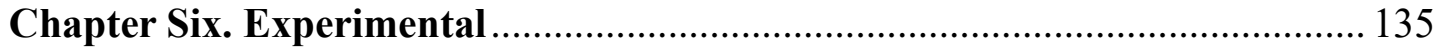

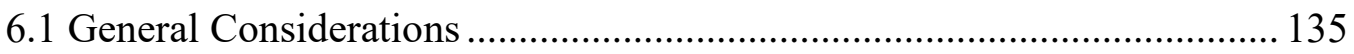

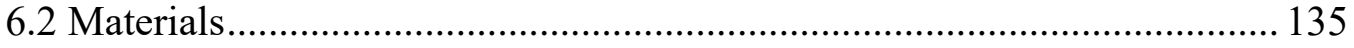

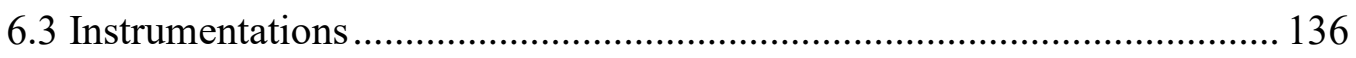

6.4 Synthesis of Lewis Base Compounds .................................................... 137

6.5 Synthesis of Lewis Acid Compounds ….................................................. 141

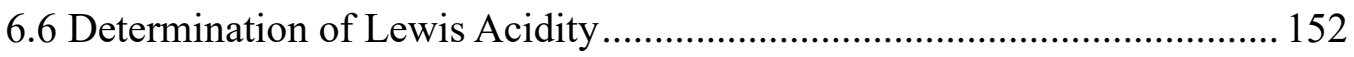

6.7 Determination of Lewis Basicity …................................................... 153

6.8 RAFT Polymerisations of Monomers …................................................ 155

6.9 Network Formation from Polymeric FLPs .......................................... 156

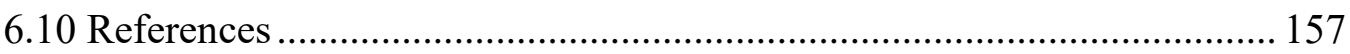




\section{List of Abbreviations}

2-APB

3-BSt

AIBN

AN

ATRP

$\mathrm{BBr}_{3}$

$\mathrm{BCF}$

$\mathrm{BCl}_{3}$

$\mathrm{BF}_{3}$

CAN

CDB

$\mathrm{CDCl}_{3}$

$\left(\mathrm{C}_{6} \mathrm{~F}_{5}\right)_{2} \mathrm{BF}$

(C6F5) MgBr

CLP

$\mathrm{CO}_{2}$

Đ

DCM

DEAD

DMSO

DN

$\mathrm{dn} / \mathrm{dc}$

$\mathrm{Et}_{2} \mathrm{O}$ 2-aminoethyldiphenyl borate

4-(3-butenyl)styrene

Azobisisobutyronitile

acceptor number

atom transfer radical polymerisation

boron tribromide

tris(pentafluorophenyl)borane

boron trichloride

boron trifluoride

covalent adaptable network

cumyl dithiobenzoate

deuterated chloroform

bis(pentafluorophenyl)borane fluoride

pentafluorophenylmagnesium bromide

conventional Lewis pair

carbon dioxide

dispersity

dichloromethane

diethyl azodicarboxylate

dimethyl sulfoxide

donor number

refractive index increment

diethyl ether 
FBPh2

FLP

G'

G”

GPC

$\mathrm{H}_{2}$

$\mathrm{HB}\left(\mathrm{C}_{6} \mathrm{~F}_{5}\right)_{2}$

$\mathrm{HCl}$

IR

LA

LB

LLP

LPA

$\mathrm{MeOBPh}_{2}$

Mes2PX

MMA

$\mathrm{M}_{\mathrm{n}}$

$\mathrm{M}_{\mathrm{n}, \text { calcd. }}$

MS-EI

nBA

$\mathrm{NH}_{4} \mathrm{Cl}$

$\mathrm{NHC}$

$\mathrm{NHO}$

NMR

$\mathrm{Ph}_{2} \mathrm{PCl}$

PhMgBr

RA fluorodiphenylborane

frustrated Lewis pair

storage modulus

loss modulus

gel permission chromatography

hydrogen gas

bis(pentafluorophenyl)borane

hydrogen chloride

infrared

Lewis acid

Lewis base

Lewis pair polymerisation

Lewis pair adduct

methoxyldiphenylborane

dimesitylphosphorous halide

methyl methacrylate

number averaged molecular weight

calculated number averaged molecular weight

mass spectroscopy-electron impact

n-butyl acrylate

ammonia chloride

N-heterocyclic carbene

N-heterocyclic olefin

nuclear magnetic resonance

diphenylphosphorous chloride

phenylmagnesium bromide

relative acidity 
RAFT

$\mathrm{SbCl}_{5}$

SEM

$\left(\mathrm{StF}_{4}\right) \mathrm{B}\left(\mathrm{C}_{6} \mathrm{~F}_{5}\right)_{2}$

$\mathrm{StB}(\mathrm{OMe})_{2}$

StBBr2

StBPh2

$\mathrm{StBPh}_{2} \cdot \mathbf{N H}_{3}$

$\mathrm{StF}_{4} \mathrm{Br}$

StMgBr

StMgCl

StP(=Se)Mes2

$\mathrm{StP}(=\mathrm{Se}) \mathrm{Ph}_{2}$

StPMes2

StPPh2

StSiMe3

THF

TIPT

Tol

TTS

UPy

$\omega$ reversible addition fragmentation chain-transfer

antimony pentachloride

scanning electron microscopy

2,3,5,6-tetrafluorosytyrl-bis-

pentafluorophenylborane

dimethoxyborylstyrene

dibromoborylstyrene

4-styryl-diphenylborane

4-styryl-diphenylborane ammoniate

2,3,5,6-tetrafluoro-4-bromostyrene

4-styrylmagnesium bromide

4-styrylmagnesium chloride

4-styryl-dimesitylphosphine selenide

4-styryl-diphenylphosphine selenide

4-styryl-dimesitylphosphine

4-styryl-diphenylphosphine

4-styryl-trimethylsilane

tetrahydrofuran

1,3,5-triisopropyl-2,4,6-trioxane

toluene

time-temperature superposition

ureidopyrimidinone

angular frequency 


\section{List of Figures}

\section{Chapter One}

Figure 1.1 Schematic representation of (A) Brønsted and Lowry acid-base theory and

(B) Lewis acid-base theory.

Figure 1.2 (A) Conventional Lewis pair (CLP) and (B) Frustrated Lewis pair (FLP) 4

Figure 1.3 Relationship between the values of $\mathrm{pK}_{\mathrm{b}}$ and ${ }^{1 / J} \mathrm{PSe}$ of triaryl-/trialkylphosphines by Beckmann et al ........................................................... 9

Figure 1.4 Selected examples of product of $\mathrm{CO}_{2}$ activation by FLPs.

Figure 1.5 Figure to represent the structure of (A) cluster and multiplet; (B) ionomer with ordered cluster and crystallinity; (C) ionomer with disordered cluster and crystallinity and (D) melted ionomer.

Figure 1.6 $\pi$-stacking interaction based crosslinked organogel by Colquhoun et al. 26

Figure 1.7 Quadruple hydrogen bonding crosslinked polymer by Meijer et al. .......27

Figure 1.8 "Lock and Key" Van der Waals force crosslinked self-healing polymers by Urban et al. 30

\section{Chapter Two}

Figure 2.1 Lewis basicity determination using ${ }^{31} \mathrm{P}$ NMR spectroscopy. 53

Figure 2.2 ${ }^{1} \mathrm{H}$ NMR spectra to show the boron-silicon exchange reactions between StSiMe 3 and boron trihalides. (A) StSiMes before reaction; (B) $1 \mathrm{~h}$ after mixing of StSiMe 3 and $\mathbf{B B r} 3$ in DCM at $-78^{\circ} \mathrm{C}$; (C) $168 \mathrm{~h}$ after mixing of StSiMe 3 and $\mathbf{B C l}_{3}$ in toluene at r.t. and (D) $1 \mathrm{~h}$ after mixing of StSiMez and $\mathbf{B B r}_{3}$ in toluene at r.t. .57 
Figure 2.3 (A) ${ }^{1} \mathrm{H}$ NMR spectra and (B) overlapped ${ }^{11}$ B NMR spectra of $\mathbf{S t B P h}$ and $\mathrm{StBPh}_{2} \cdot \mathrm{NH}_{3}$ 63

Figure 2.4 Determination of Lewis acidity of $\mathbf{S t B P h}$ 65

\section{Chapter Three}

Figure 3.1 (A) Schematic representation of the binding of PhPMes2, BPh 3 and DEAD; (B) ${ }^{31} \mathrm{P}$ NMR spectrum of the binding experiment; (C) overlapped ${ }^{11} \mathrm{~B}$ NMR spectra of unbound $\mathbf{B P h}_{3}$ (grey line) and the binding experiment

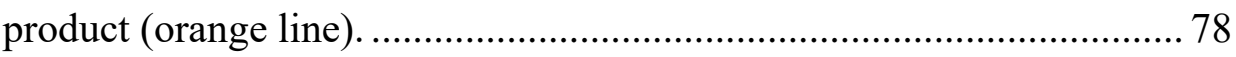

Figure 3.2 Variable-temperature ${ }^{31} \mathrm{P}$ NMR spectra of PhPMes2 $\cdot$ DEAD $\cdot \mathbf{B P h} 3$. The numbers under each spectrum show the relative integrals of the peaks in the green and yellow area................................................................ 79

Figure 3.3 Variable-temperature ${ }^{11} \mathrm{~B}$ NMR spectra of PhPMes2 $\cdot$ DEAD $\cdot \mathbf{B P h} 3 \ldots . . . . .81$

Figure 3.4 Gelation experiment using Copolymers P1 and B1, and DEAD. ........... 83

Figure 3.5 Shrinkage in the volume of the P1B1 gel after injection of DEAD....... 83

Figure 3.6 Products of the attempted synthesis of gel (A) P2B2 and (B) P3B3 ...... 85

Figure 3.7 IR spectra of DEAD (top) and poly(FLP) gel P1B1 (bottom). The carbonyl bands are denoted with red dots. 86

Figure 3.8 Swelling ratio of gel P1B1 (blue) and P1B3 (green). 87

Figure 3.9 SEM images of poly(FLP) gels (A) P1B1; (B) higher magnification of P1B1; (C) P1B2; (D) higher magnification of P1B2; (E) P1B3; (F) higher magnification of P1B3. 88

Figure 3.10 Graph to show the rheology method used in this project. 90

Figure 3.11 Frequency-sweep test of gel P1B1 at $0.1 \%$ strain, $22{ }^{\circ} \mathrm{C}$ 90

Figure 3.12 Overlapped frequency-sweep curves of poly(FLP) gel at different temperatures. .92

Figure 3.13 Time-temperature superposition graph of poly(FLP) gel. .93 
Figure 3.14 Rupture of poly(FLP) gel by heating. 94

Figure 3.15 Temperature-ramp rheology test for poly(FLP) gel............................ 94

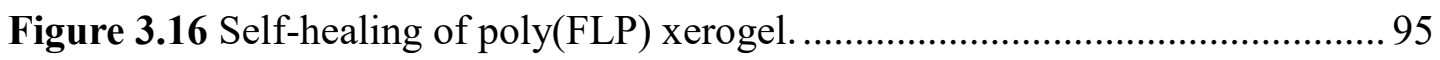

Figure 3.17 Self-healing of fully swollen poly(FLP) gel. ..................................... 96

Figure 3.18 Rheology characterisation of self-healing of poly(FLP) gel. 97

Figure 3.19 (A) Schematic representation of binding experiments using equivalent amounts of StPPh2, StBPh2 and DEAD; overlapped (B) ${ }^{31}$ P NMR spectra and $(\mathbf{C}){ }^{11} \mathrm{~B}$ NMR spectra of binding reaction mixture (blue and orange colour) and unbound monomers (grey). 99

\section{Chapter Four}

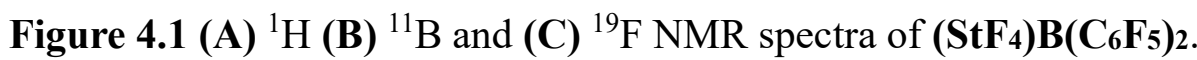
110

Figure 4.2 ${ }^{31} \mathrm{P}$ NMR spectrum to determine the Lewis acidity of $\left(\mathbf{S t F}_{4}\right) \mathbf{B}\left(\mathbf{C}_{6} \mathbf{F}_{5}\right)_{2} .111$

Figure 4.3 Solutions of boron and phosphorus-containing monomers (A) before mixing (Left: (StF4)B(C6F5)2; Right: StPMes2) and (B) after mixing. 112

Figure 4.4 Gelation experiment of 2nd generation poly(FLP). (A) Solution of poly[Styrene-co-(StF4)B(C6 $\left.\left.\mathbf{C}_{5}\right)_{2}\right]$ (left) and poly(styrene-co-StPMes2) (right). (B) The mixture of the two copolymers. 114

Figure 4.5 Thermal responsiveness of the 2nd gen. poly(FLP) gel. (A) Before heating; (B) turning to polymer solution after heating; (C) cooling down by icebath; (D) recovered gel. 116

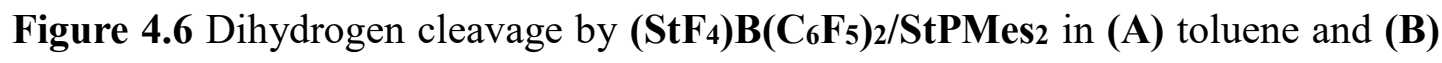
in bromobenzene, and (C) by BCF/StPMes2 in bromobenzene. 118

Figure 4.7 (A) ${ }^{31} \mathrm{P}\left\{{ }^{1} \mathrm{H}\right\}$, (B) ${ }^{1} \mathrm{H}$-coupled ${ }^{31} \mathrm{P}$, and (C) ${ }^{11} \mathrm{~B}\left\{{ }^{1} \mathrm{H}\right\}$ NMR spectra of StPMes2 (blue line) and $\left(\mathbf{S t F}_{4}\right) \mathbf{B}\left(\mathbf{C}_{6} \mathbf{F}_{5}\right)_{2}$ (orange line) and their product from dihydrogen cleavage (green line). 119 


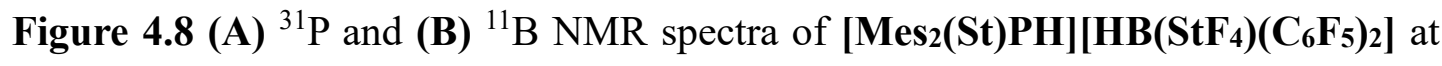

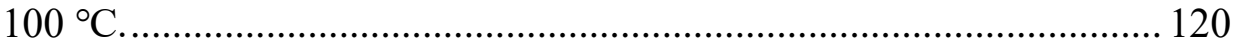

Figure 4.9 Overlapped ${ }^{11} \mathrm{~B}$ NMR spectrum to show the hydroboration reaction.... 123

Figure 4.10 Overlapped ${ }^{11} \mathrm{~B}$ NMR spectra to monitor the reaction between $\mathrm{HCl}$ and $\mathrm{StB}\left(4-\mathrm{CF}_{3}-\mathrm{Ph}_{2}\right) \cdot \mathrm{NH}_{3}$ 125 


\section{List of Schemes}

\section{Chapter One}

Scheme 1.1 Frustrated Lewis pairs developed by Stephan et al................................ 3

Scheme 1.2 Gutmann's scale to approximate solvent donicity of common laboratory solvents by complexation with $\mathrm{SbCl}_{5}$

Scheme 1.3 Gal and Maria's $\mathrm{BF}_{3}$ affinity scale to quantify the relative Lewis basicity of compounds. 8

Scheme 1.4 Schematic representation of (A) Gutmann-Beckett method and (B) Childs method (irrelevant peaks are hidden).

Scheme 1.5 Selected FLP complexes formed with small molecules. 13

Scheme 1.6 Selected examples of FLP hydrogenation substrates. Reductions of imine (top), silyl enol ether (middle), and enamine (bottom). 14

Scheme 1.7 Mechanistic representation of FLP-catalysed hydrogenation of imines. (A) Hydrogenation by LA/LB pair. (B) Self-catalysed reduction of imine as a base partner of an added LA.

Scheme 1.8 Lewis Pair Polymerisation of a polar conjugated olefin monomer. 19

Scheme 1.9 Schematic representations of selected examples of covalent dynamic crosslinks. Wavy bonds represent polymer backbones.

Scheme 1.10 Schematic representation of CLP supramolecular polymer made by (A) Wagner et al. and (B)-(C) Severin et al.

Scheme 1.11 Schematic representations of conventional Lewis pair crosslinked supramolecular polymers by Jäkle et al. .34 


\section{Chapter Two}

Scheme 2.1 Schematic representations of (A) hybridisation of boron in an organoboron compound and (B) conformational change of organoboron when coordinated by a Lewis base. 44

Scheme 2.2 Common organoboron compounds. ${ }^{1}$................................................... 44

Scheme 2.3 Common organophosphorus compounds......................................... 46

Scheme 2.4 Structures of target phosphorus and boron-containing monomers. ....... 48

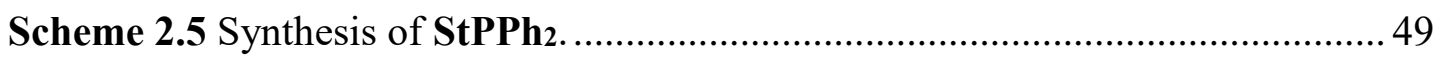

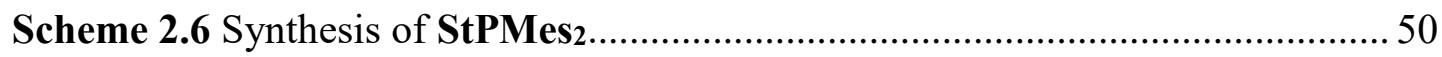

Scheme 2.7 Synthesis of $\operatorname{StP}(=\operatorname{Se}) \mathbf{P h} 2$ and $\operatorname{StP}(=$ Se)Mes2................................... 52

Scheme 2.8 Attempted synthesis of StBPh2 via exchange reactions. ...................... 55

Scheme 2.9 Structures of FBPh 2, MeOBPh$_{2}$, and 2-APB. .................................... 58

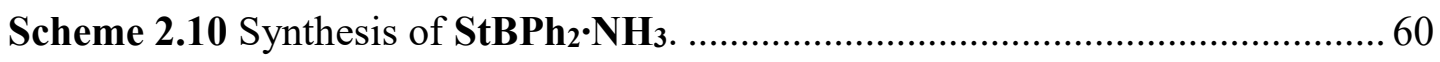

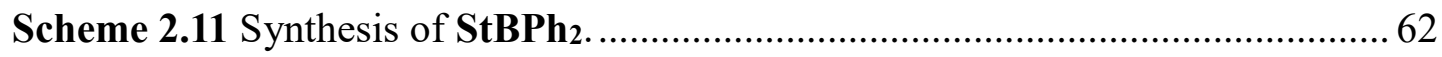

Scheme 2.12 Triarylborane synthesised using coordination-recovery strategy. ....... 64

Scheme 2.13 Side reactions between phosphine monomer and polymer chain end. 66

Scheme 2. 14 RAFT copolymerisation of phosphine monomers and styrene...........67

Scheme 2.15 RAFT copolymerisations of StBPh2 and styrene. ..............................69

\section{Chapter Three}

Scheme 3.1 Binding between StBPh2 and StPPh2........................................... 75

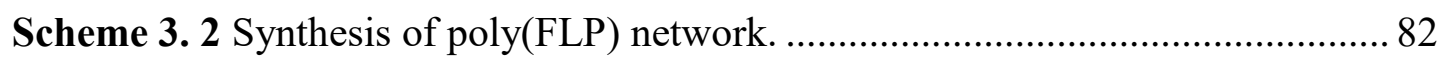

Scheme 3.3 Gelation experiment using P4, B1 and DEAD............................... 100

Scheme 3.4 Proposed mechanism for crosslinking via (A) replacement and (B) ringopening of THF by phosphorus-DEAD adduct. 101 


\section{Chapter Four}

Scheme 4.1 Fluorinated-aryl substituted borane-containing polymers reported by Yan et al. 105

Scheme 4.2 Synthetic route for fluorinated-aryl substituted borane $\left(\mathrm{StF}_{4}\right) \mathbf{B}_{(}\left(\mathrm{C}_{6} \mathbf{F}_{5}\right)_{2}$. 107

Scheme 4.3 Synthesis of $\mathrm{StF}_{4} \mathrm{Br}$. 108

Scheme 4.4 Attempted post-polymerisation modification reaction to synthesise

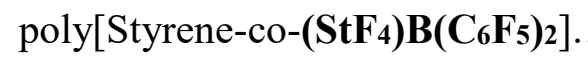
116

Scheme 4.5 Hydrogenation of N-benzylidene-tert-butylamine. 120

Scheme 4.6 Hydroboration reaction to synthesis boron-containing monomer. 122

Scheme 4.7 Structures of trifluoromethyl-substituted triarylborane monomer and their precursors. 124

\section{Chapter Five}

Scheme 5.1 Structure of the possible developed boron and phosphorus-containing monomers. 133

Scheme 5.2 Catalytic hydrogenation of $\mathrm{CO}_{2}$ using poly(FLP) system. 134 


\section{List of Tables}

Table 2.1 RAFT copolymerisation of styrene and StPMes2, $\mathrm{T}=110{ }^{\circ} \mathrm{C}$.................6 68

Table 2.2 RAFT copolymerisations of styrene and $\mathbf{S t B P h}, \mathrm{T}=110{ }^{\circ} \mathrm{C} \ldots \ldots \ldots \ldots \ldots . . . . . . . . .70$ 


\section{Publications}

Wang, M.; Nudelman, F.; Matthes, R. R.; Shaver, M. P., Frustrated Lewis Pair

Polymers as Responsive Self-Healing Gels, J. Am. Chem. Soc. 2017, 139 (40), 1423214236. DOI: $10.1021 /$ jacs. $7 b 07725$

Yolsal, U.; Wang, M.; Royar, J. R.; Shaver, M. P., Rheological Characterization of

Polymeric Frustrated Lewis Pair Networks, 2019. Accepted. DOI:

10.1021/acs.macromol.9b00271 


\section{Chapter One. Frustrated Lewis Pairs and Dynamic Crosslinked Polymeric Networks}

\subsection{Introduction to Frustrated Lewis Pairs}

\subsubsection{Lewis Pair Adducts and Frustrated Lewis Pairs}

\subsubsection{History of Acid/Bases Theory}

As one of the most important classes of chemicals, acids and bases have been widely studied and used for a very long time and have an interesting history. Since the discovery of these compounds, people have suggested many concepts about acids and bases to try to understand the nature of these compounds, as knowledge and experimental techniques have advanced, however, most of these have subsequently been disproven or have been significantly modified. The first scientific definitions of acids and bases were established in 1661 by Robert Boyle, who described an acid as a class of corrosive compounds capable of dissolving some metals and turning the colour of litmus solution to red. A base was defined as a slippery compound that can turn litmus blue. Boyle also mentioned that acids and bases will lose their respective strengths when combined, i.e. become neutralised. This instructive and seminal work provided an easy way for people to identify protic acids and bases using litmus solution, which is still being used today. In 1884, Svante Arrhenius defined acids and bases as classes of compounds that can dissociate protons and hydroxide ions, respectively, in water. ${ }^{1}$ This definition explains the fact that acids and bases can neutralise each other and allows for the quantification of the strength of an acid or a base. It can only be applied for water-mediated systems, however. Also, some well-known bases that do 
not have hydroxide anions like $\mathrm{Na}_{2} \mathrm{CO}_{3}$ are excluded from the concept. As an improvement, Johannes N. Brønsted and Thomas M. Lowry each independently introduced their definition of an acid and base in $1923 .{ }^{1-2}$ From their theory, an acid is defined as a compound that can donate protons to form its conjugate base, while a base is defined as one that can receive protons to form its conjugate acid (Figure 1.1 (A)). ${ }^{2-}$ ${ }^{3}$ This definition dramatically increased the scope of the acid/base because it can be applied to non-aqueous systems and hence has been widely used by chemists until today.

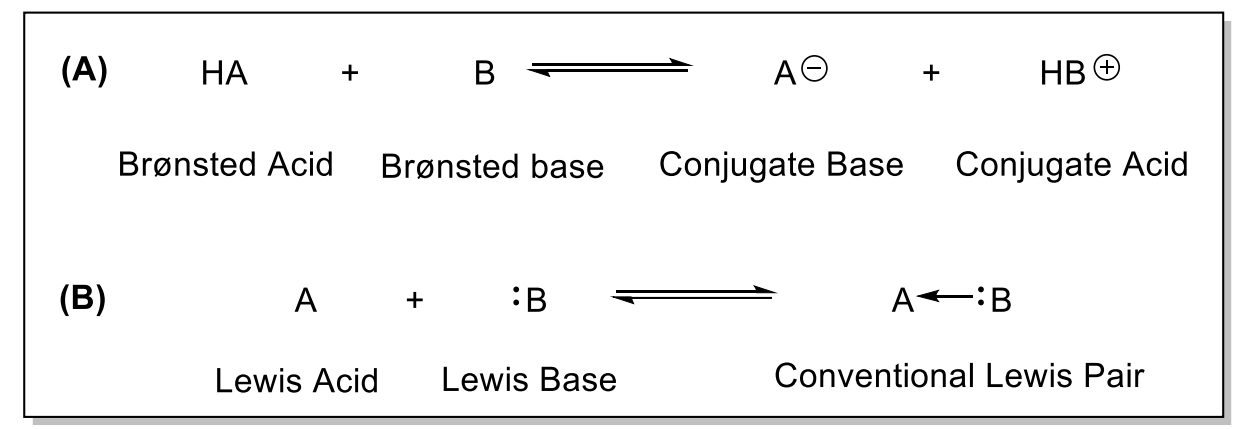

Figure 1.1 Schematic representation of (A) Brønsted and Lowry acid-base theory and (B) Lewis acid-base theory.

While Brønsted and Lowry's theory greatly expanded the concept of acids and bases, it still does not provide a comprehensive overview of all acids and bases. For example, an aprotic compound, boron trifluoride, is not classified as a Brøsted acid but can neutralise a known Brønsted base, such as $\mathrm{NH}_{3}$. Apparently, a different reaction mechanism is involved, and thus a different acid/base concept is required for these exceptions. In the same year as Brønsted's and Lowry's works were published, Gilbert N. Lewis proposed another method to describe acids and bases. He defined acids as compounds that are able to accept a pair of electrons, and bases as compounds that can donate a pair of electrons. ${ }^{4}$ When a Lewis base donates its lone pair to a Lewis acid, a dative covalent bond is formed between them, and both the acid and base will lose their reactivity as they quench each other. The resultant stable product that is formed is normally called a Lewis pair adduct (LPA), or conventional Lewis pair (CLP), as 
shown in Figure 1.1 (B). Lewis' concept reveals the most fundamental nature of acids and bases, wherein a reaction between an acid and a base depends on the tendency of electron movements. Lewis acids and bases are therefore incorporated into nearly all synthetic processes, although they are more commonly referred to as electrophile and nucleophile, respectively, in organic chemistry. Compared to the other acid-base concepts mentioned above, the Lewis definition has the largest scope. ${ }^{2}$

\subsubsection{Discovery of Frustrated Lewis Pairs}

From the extensive research in Lewis acids/bases, people have discovered many examples of sterically encumbered Lewis pair, which the Lewis acid and base centres cannot bind together. ${ }^{5-6}$ Although this type of Lewis pairs showed unique property and applications in synthetic chemistry, its systematic investigations were lacking until Stephan et al. developed their first example of sterically encumbered Lewis pair in $2006 .^{7-8}$ Their research began by exploring the borane and borate salts activated in olefin polymerisation. ${ }^{7}$ As they explored the reactions between phosphines and Lewis acid activators, although in most cases conventional Lewis pair adducts were formed without any doubt, their attention was drawn to some unusual products that were obtained when using sterically bulky phosphines (Scheme 1.1). ${ }^{7}$

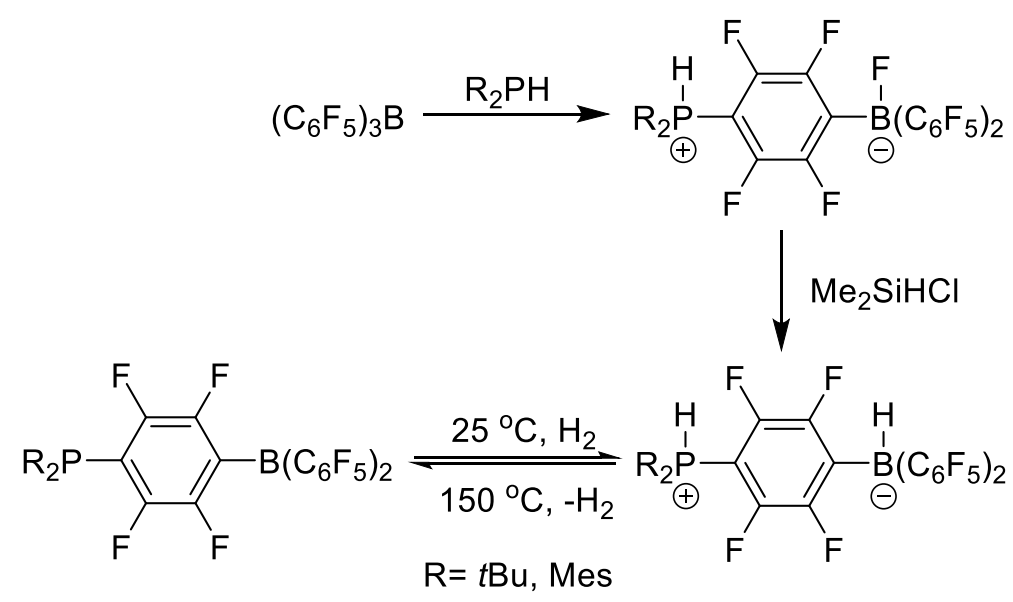

Scheme 1.1 Frustrated Lewis pairs developed by Stephan et al..$^{7-8}$ 
By a nucleophilic aromatic substitution at the para-position of tris(pentafluorophenyl)borane $(\mathrm{BCF}), \mathrm{R}_{2} \mathrm{PH}$ was connected to form the product $\left[\mathrm{R}_{2} \mathrm{PH}\left(\mathrm{C}_{6} \mathrm{~F}_{4}\right) \mathrm{BF}\left(\mathrm{C}_{6} \mathrm{~F}_{5}\right)_{2}\right]$, where $\mathrm{R}$ is a bulky moiety such as tertiary butyl or a mesitylene group. ${ }^{7}$ The fluorine atom that was transferred to the boron atom was replaced by a hydride using $\mathrm{Me}_{2} \mathrm{SiHCl}^{7}$ The unusual product obtained, $\left[\mathrm{R}_{2} \mathrm{PH}\left(\mathrm{C}_{6} \mathrm{~F}_{4}\right) \mathrm{BH}\left(\mathrm{C}_{6} \mathrm{~F}_{5}\right)_{2}\right]$, attracted more attention since it contained a proton and a hydride that were attached on both the phosphonium and borane centres at the same time. ${ }^{7}$ Stephan et al. found that hydrogen gas could be released from this product when the temperature was elevated to $150{ }^{\circ} \mathrm{C}$ and, surprisingly, the resultant product $\left[\mathrm{R}_{2} \mathrm{P}\left(\mathrm{C}_{6} \mathrm{~F}_{4}\right) \mathrm{B}\left(\mathrm{C}_{6} \mathrm{~F}_{5}\right)_{2}\right]$ could recapture dihydrogen in a heterolytic manner as soon as the temperature decreased..$^{7-8}$ It was also found that the substituents attached to phosphorous and boron atoms were sufficiently sterically bulky to prevent intermolecular binding. These phenomena suggested that the phosphorous and boron centres maintained their reactivity towards each other through the steric hindrance between them, making them able to cleave chemical bonding. Stephan et al. named this kind of complex a Frustrated Lewis Pair (FLP), as shown in Figure 1.2.

(A)

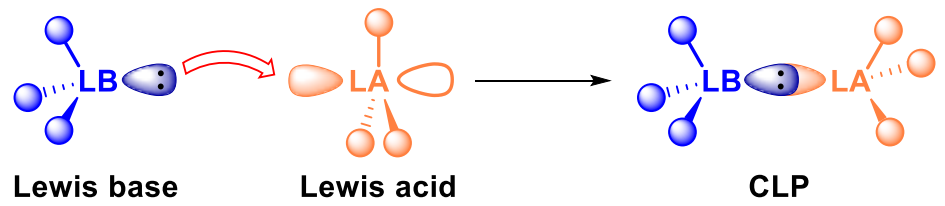

(B)
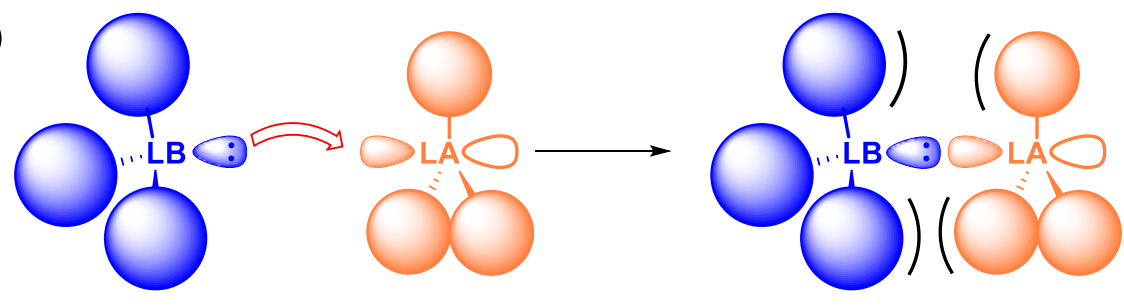

Lewis base

Lewis acid

FLP

Figure 1.2 (A) Conventional Lewis pair (CLP) and (B) Frustrated Lewis pair (FLP)

Unlike CLPs, the large bulky moieties around the binding centres in FLPs prevent the formation of a dative covalent bond between the Lewis acid and base. This makes the 
Lewis acid and base unquenched, hence giving them the ability to activate small molecules. Apart from the first example, Stephan et al. also prepared other FLPs by simple combinations of non-linked phosphines and boranes, which can also achieve the heterolytic cleavage of dihydrogen. ${ }^{9}$ They also found that the substituents on boron and phosphorous atoms could affect the reactivity of the resultant FLPs. After the first report, the field of FLPs attracted significant attention and quickly became a noteworthy area in inorganic chemistry due to its potential in respect to cleaving chemical bonds. ${ }^{7}$ Later researchers focused on developing novel FLPs and studying their activities towards dihydrogen and other small molecules, as is discussed in later sections.

\subsubsection{FLP reactivity and Lewis Basicity/Acidity}

After the pioneering work by Stephan et al., more FLPs have been prepared using various combinations of Lewis bases/acids, and thence investigating their reactivity. Many researchers have expanded the scope of FLP chemistry beyond phosphorus and boron, mainly from Group 13 and Group 15 elements, but also including transition metals. As a simple guideline, the reactivity of an FLP relates to the strength of its individual Lewis acid and base, along together with the steric hindrance around them. ${ }^{8}$ ${ }^{10}$ The understanding of the strengths of the respective Lewis base and acid is therefore integral to predicting their properties. Although the concept of FLPs is a relatively new field in chemistry, the chemistry of the major components, i.e. the Lewis acids and Lewis bases, are well established. While several different methods have been suggested to define these components, there is still, however, no universal methodology to define and quantify either the strength of Lewis basicity or Lewis acidity. ${ }^{11-12}$ This problem mainly comes from the limited scope of application of each method of definition. ${ }^{11}$ In contrast to Brønsted acids and bases, where the proton in equilibrium is an intrinsic reference to define their strengths, ${ }^{11}$ there is no such a 
universal intrinsic reference for either Lewis bases or acids. In many cases, it is necessary to use more than one reference to explain the relative Lewis strengths of a series of compounds. The following sections will introduce the major Lewis basicity/acidity scales that are in widespread use today.

\subsubsection{Lewis Basicity Scales}

One of the earliest attempts to measure Lewis basicity was established by Gutmann in 1966, mainly to examine the Lewis basicity of common laboratory solvents (Scheme 1.2). ${ }^{11,13-14}$ This method involves the use of a strong Lewis acid, antimony pentachloride $\left(\mathrm{SbCl}_{5}\right)$ as the acid reference, and defining the donicity of a certain solvent in terms of the donor number (DN). DN refers to the negative enthalpy change when this solvent binds to $\mathrm{SbCl}_{5}$ with 1:1 stoichiometry at low concentrations in a noncoordinating solvent, normally 1,2-dichloroethane. ${ }^{11,13-14}$ One requirement of a noncoordinating solvent is to minimise the impact on test results as it has a DN of zero. ${ }^{11}$, ${ }^{13}$ Since the value of $\mathbf{D N}$ is actually a reflection of the inherent base- $\mathrm{SbCl}_{5}$ bond strength, it can be used for Lewis basicity approximation. ${ }^{14}$

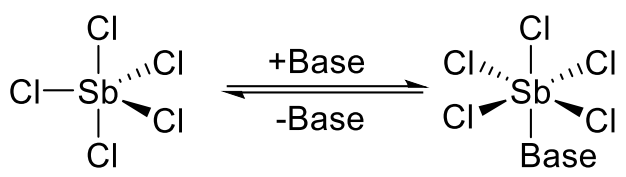

Scheme 1.2 Gutmann's scale to approximate solvent donicity of common laboratory solvents by complexation with $\mathrm{SbCl}_{5} .{ }^{11,13-15}$

While such a system is somewhat successful in giving the relative Lewis basicity of a series of compounds, there are still some limitations: (i) the scope of eligible bases is limited to mainly oxygen-derivatives. ${ }^{11,15}$ There is a lack of nitrogen-, carbon $\pi$-, halogen- and sulphur-derivative bases. ${ }^{11,15}$ For example, $\mathrm{SbCl}_{5}$ cannot test amine bases as it can catalyse the reaction between chlorinated solvents and amines. ${ }^{15}$ Also, protic 
compounds like water, alcohols and protic amides cannot be measured by this method directly. ${ }^{16}$ (ii) incomplete complexation reaction between $\mathrm{SbCl}_{5}$ and weak bases may lead to unreasonable results. ${ }^{11,15}$ (iii) calorimetry is not readily available in most chemistry laboratories since it requires specialised equipment and expertise, hence Gutmann's DN method is relatively inconvenient. ${ }^{17}$ (iv) complicated inter shell electron configurations lead to hard and costly quantum mechanical calculations. ${ }^{11,15}$ (v) the solvent system is mainly limited to 1,2-dichloroethane since $\mathrm{SbCl}_{5}$-base complexes have limited solubility and alternative solvents frequently cannot give reliable results. ${ }^{15}$ (vi) Even though, normally, a base is supposed to be more basic if it can form a more stable product with a certain acid, one fact that should be kept in mind is that the hard/soft nature of the reference acid has a great effect on the stability of the resultant complexation product. According to Pearson's hard and soft acids and bases (HSAB) theory, ${ }^{18} \mathrm{SbCl}_{5}$ is a hard Lewis acid, hence it normally forms a more stable product with hard bases. This means that the basicity of soft bases will be underestimated in the Gutmann method. This is also a reason why the quantitative estimation of both the relative Lewis basicity and the Lewis acidity is very challenging work. $^{12}$

Alternative, and more broad scope, measures of Lewis acid/base strength include the $\mathrm{BF}_{3}$ scale. The $\mathrm{BF}_{3}$ affinity is defined as the negative enthalpy change of the complexation reaction between a base and the reference acid, in this case gaseous $\mathrm{BF}_{3}$, in dichloromethane (DCM) (Scheme 1.3). ${ }^{11}, 19$ This scale was established by Gal and Maria et al. in 1971. ${ }^{11,19}$ Compared to Gutmann's donor number, this system broadens the scope of available bases because boron trifluoride can give clear complexation reactions and shows less side reactivity towards either solvent or base. ${ }^{11,19}$ Also, thanks to the simple electronic configuration of $\mathrm{BF}_{3}$, computations become much more facile. ${ }^{11,19}$ 


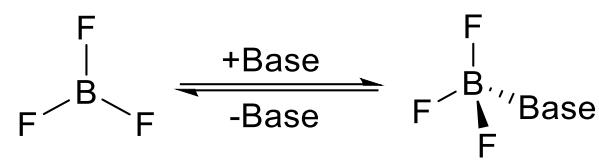

Scheme 1.3 Gal and Maria's $\mathrm{BF}_{3}$ affinity scale to quantify the relative Lewis basicity of compounds. ${ }^{11,19}$

Although, as an improved version of Gutmann's donor number, the $\mathrm{BF}_{3}$ affinity scale shows some improvements in testing a chemical's Lewis basicity, there are still several practical problems: (i) The system is restricted to the use of gaseous $\mathrm{BF}_{3}$ and $\mathrm{DCM}$ for technical reasons, however DCM itself can be regarded as a weak Lewis acid which can slightly affect the results; ${ }^{19}$ (ii) the measurement still relies on specialised equipment, which is not readily available in most labs; (iii) while the scope of the bases that can be tested through this scale is much greater, especially the complement of nitrogen-containing bases, still many classes of bases are excluded, e.g. phosphines; ${ }^{11}$ (iv) similar to $\mathrm{SbCl}_{5}, \mathrm{BF}_{3}$ also suffers from the hard/soft nature of the tested bases. As a hard Lewis acid, any soft base tested by it will be underestimated in Lewis strength.

As mentioned above, both the $\mathrm{SbCl}_{5}$ and $\mathrm{BF}_{3}$ affinity scales have a common problem, which is the ease of measurement. The most convenient, easy and quick way is to use common lab techniques to probe the relative basicity of a series of compounds rather than using a machine like a calorimeter, which is not very accessible to user-friendly for non-experienced workers. In the field of organophosphorus chemistry, researchers have found an easy way of using NMR spectroscopy to predict the $\sigma$-donating ability of phosphine compounds by using their selenide derivatives. The related work can be traced back to the 1970s when many researchers had noted that the ${ }^{1} \mathrm{~J}$ PSe value in different phosphine selenide compounds has a very broad range and is significantly affected by the substituents attached to the phosphorus atom. This feature allows this coupling constant to be used as a sensitive probe of the inductive and steric effects of the attached substituents. ${ }^{20-22}$ The method involves the transformation of the target 
phosphine into its selenide derivative via a simple single-step reaction, and thence the measurement of the coupling constant between phosphorus and selenium atoms ${ }^{1} \mathrm{~J}$ PSe in the resultant compound. ${ }^{21-24}$ As a general trend, the more electron-withdrawing substituents will cause the phosphorus lone pair to have more s-character, and will normally result in a larger ${ }^{1} \mathrm{~J}$ PSe value. ${ }^{20,22,24}$ Hence the ${ }^{1} \mathrm{~J}$ PSe value is inversely proportional to the $\sigma$-donating ability of the parent phosphine compounds. ${ }^{22,24}$ This phenomenon can be intuitively shown by a plot of the $\mathrm{pK}_{\mathrm{b}}$ values of a series of phosphine compounds vs the ${ }^{1} J$ PSe values of their selenide derivatives, as shown below in Figure 1.3. ${ }^{23}$

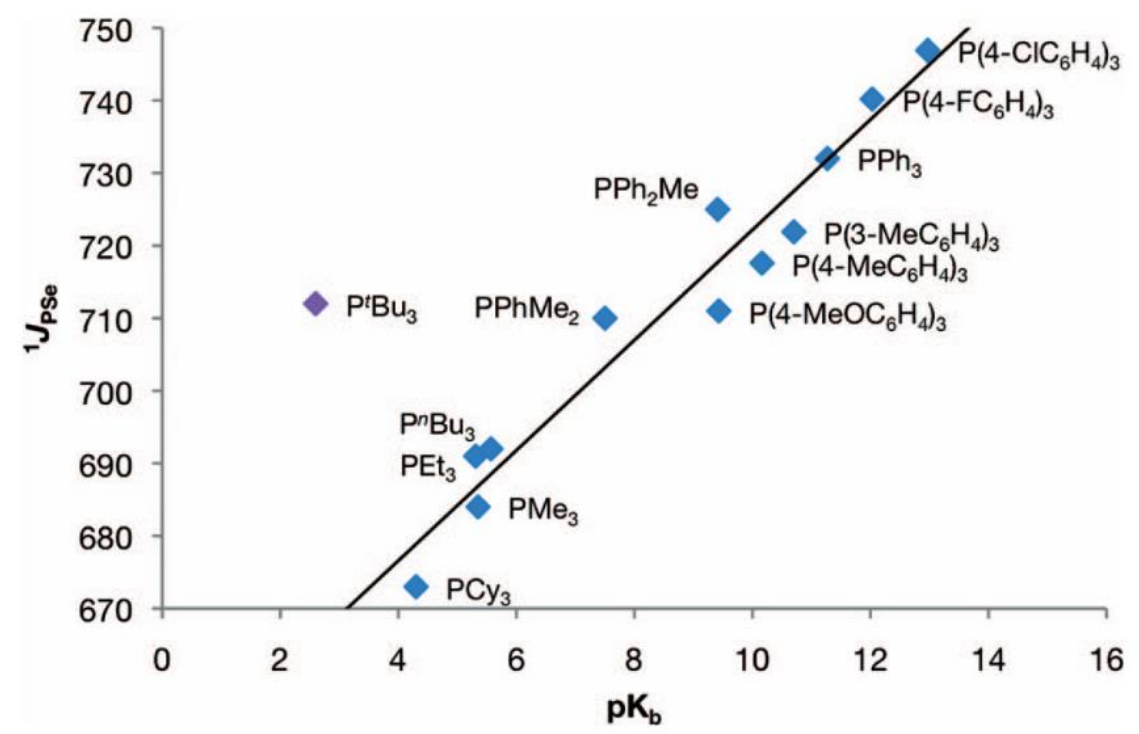

Figure 1.3 Relationship between the values of $\mathrm{pK}_{\mathrm{b}}$ and ${ }^{1 / J} \mathrm{PSe}$ of triaryl-/trialkylphosphines by Beckmann et al. ${ }^{23}$

While this method of predicting Lewis basicity is limited in organophosphorus compounds, it is easy and quick to perform and thus can be reasonably applied to the phosphorus-containing monomers mentioned in this thesis. In future experiments, other classes of Lewis base-containing monomers might be developed and a calorimetric method may be required to definitively determine the monomers' relative basicity. 


\subsubsection{Lewis Acidity Scales}

As mentioned in the previous section, the quantification of Lewis basicity suffers from the hard/soft nature of the reference acid selected. Similarly, the determination of the relative acidity of a certain Lewis acid faces similar challenges. A real example is to consider the relative acidity of $\mathrm{BH}_{3}$ and $\mathrm{BF}_{3}$, where $\mathrm{BF}_{3}$ is normally thought to be a stronger acid since its boron atom is attached with more electron-withdrawing fluorine atoms. ${ }^{12}$ If reacting with a soft base, however, such as a thioether, the softer $\mathrm{BH}_{3}$ will be determined as a stronger acid as it can form stronger complexes with thioethers. ${ }^{12}$ Despite this obvious disadvantage, the most widely used Lewis acidity scales still involve the use of reference bases for many historical reasons. The two most wellknown scales are the Gutmann-Beckett method and the Childs' method. Both methods have been applied to different classes of Lewis acids, and show various successes on boron compounds. ${ }^{12}$ Unlike the Lewis basicity $\mathbf{D N} / \mathrm{BF}_{3}$ scales, both the GutmannBeckett and Childs' scales use NMR spectroscopy rather than calorimetry to evaluate the strengths of the dative covalent bonds inside Lewis pair adducts, which means they are much easier to perform and generate more accurate testing results.

The Gutmann-Beckett method was initially established by Gutmann for the purpose of quantitatively evaluating the electron acceptability of solvent molecules. This was later extended to other Lewis acids by Beckett. ${ }^{13,25-26}$ As shown in Scheme 1.4 (A), the method involves using a Lewis base, triethylphosphine oxide $\mathrm{Et}_{3} \mathrm{P}=\mathrm{O}$, as a reference, and the Lewis acidity is then defined by means of the acceptor number (AN). This value relates to the change in the chemical shift of the reference molecule in ${ }^{31} \mathrm{P}$ NMR spectra. Two reference points are taken in the ${ }^{31} \mathrm{P}$ spectra to calculate the value of $\mathbf{A N}$, which is $\mathbf{A N}=0$ when $\mathrm{Et}_{3} \mathrm{P}=\mathrm{O}$ is dissolved in hexane $(\delta=41.0)$ and $\mathbf{A N}=100$ when $\mathrm{Et}_{3} \mathrm{P}=\mathrm{O}$ is dissolved in $\mathrm{SbCl}_{5}(\delta=86.1)$. Then the value of $\mathbf{A N}$ can be calculated by the equation shown below:

$$
\mathbf{A N}=2.21 \times\left(\delta_{L A \cdot E t_{3} P=O}-41.0\right)
$$


Where the ${ }^{31} \mathrm{P}$ chemical shift $\delta_{L A} \cdot E t 3 P=O$ is obtained by mixing a stoichiometric amount of $\mathrm{Et}_{3} \mathrm{P}=\mathrm{O}$ with the tested Lewis acid. There are no specific requirements for the solvent for testing, but the most widely reported ones are deuterated DCM, benzene and toluene. ${ }^{12}$

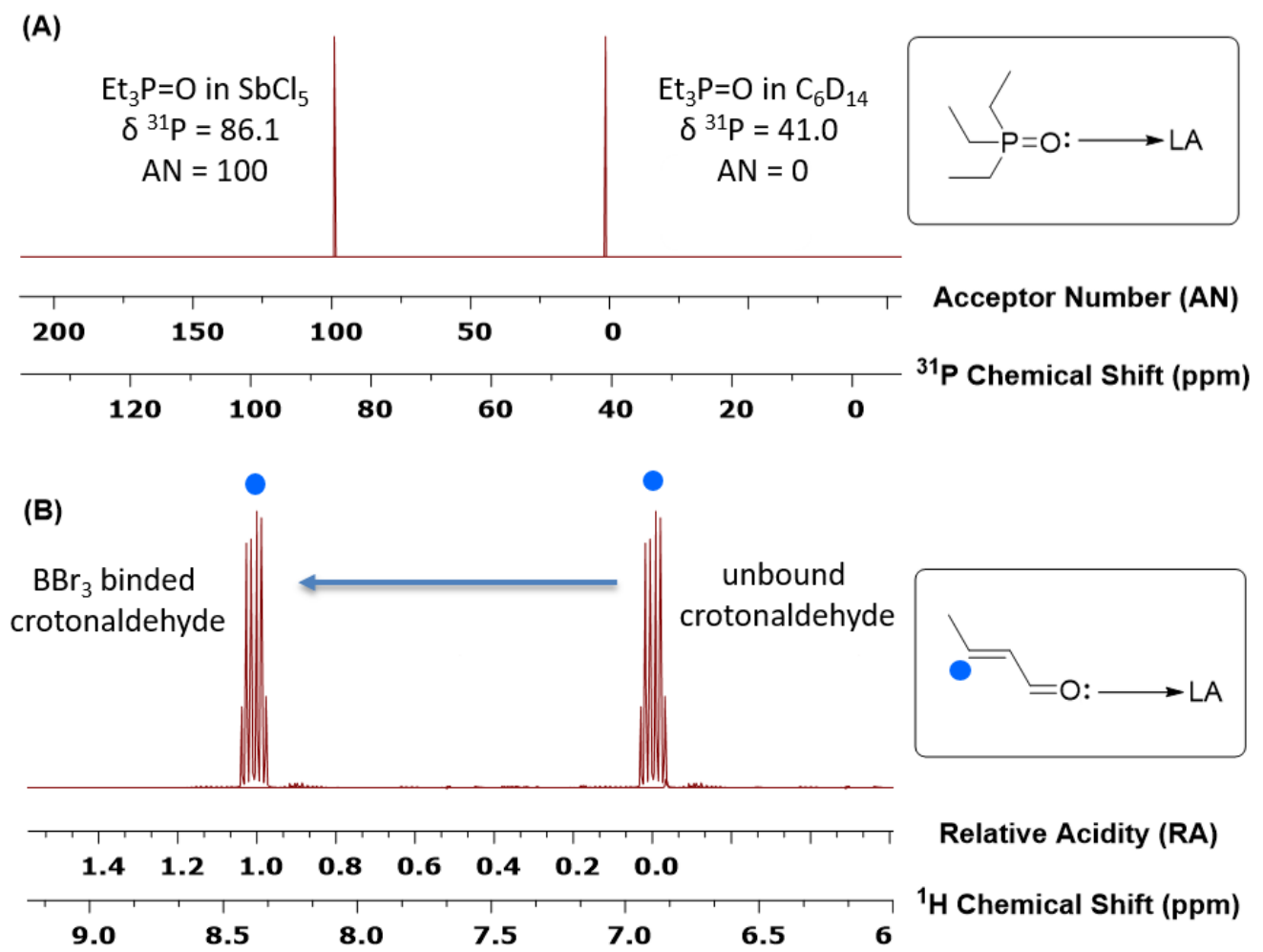

Scheme 1.4 Schematic representation of (A) Gutmann-Beckett method and (B) Childs method (irrelevant peaks are hidden).

The Childs method was established by Childs et al. in $1981 .{ }^{27}$ In an NMR study of complexations of Lewis acids and a series of unsaturated carbonyl compounds, they found that the change in chemical shifts of different protons in crotonaldehyde showed a linear relationship to each other in respect to certain boron compounds, indicating that such a molecule can be used as a reference for a Lewis acidity scale. ${ }^{27}$ The Lewis acidity of a compound is illustrated in Scheme 1.4 (B) and is defined using the equation shown below.

$$
\text { Relative acidity }(\mathbf{R A})=\frac{\Delta \delta^{1} H_{C A \cdot L A}}{\Delta \delta^{1} H_{C A \cdot B B r_{3}}}
$$


Where $\Delta \delta^{1} H_{C A \cdot L A}$ is the change in chemical shift of $\mathrm{H}_{3}$ protons of crotonaldehyde when it binds to the tested compound, and $\Delta \delta^{1} H_{C A \cdot B B r_{3}}$ is the change in chemical shift of the same protons but when crotonaldehyde binds to boron tribromide. Hence the relative Lewis acidity of boron tribromide is defined to be 1.00 . The solvent used is primarily deuterated DCM, however there are some examples in the literature of the use of deuterated chloroform.

The Gutmann-Beckett and the Childs methods show different levels of success in predicting the Lewis acidities of compounds. In the field of organoboron chemistry, including this project, the Gutmann-Beckett method has a much wider range of applications since it can cover not only the compounds with strong Lewis acidity but also several much milder Lewis acids. ${ }^{12}$ As a comparison, the Childs method normally is only sensitive to strong acids like boron halides or fluorinated triarylborane. ${ }^{12}$ Consequently, the Gutmann-Beckett method will be used in this thesis.

\subsubsection{Applications of Frustrated Lewis Pairs}

After the initial FLP discovery, research focused on their special reactivity towards small molecules, which includes carbon dioxide, ${ }^{28}$ carbon monoxide, ${ }^{29}$ nitrogen monoxide, ${ }^{30}$ sulphur dioxide ${ }^{31}$ and dihydrogen, ${ }^{32}$ olefin-containing compounds, ${ }^{33}$

molecules with special functionalities like isocyanate, ${ }^{34}$ azide, ${ }^{34}$ carbonyl, ${ }^{34-35}$ etc. Some representative FLP-small molecule complexes are shown below in Scheme 1.5. $^{7-8,36-38}$ 


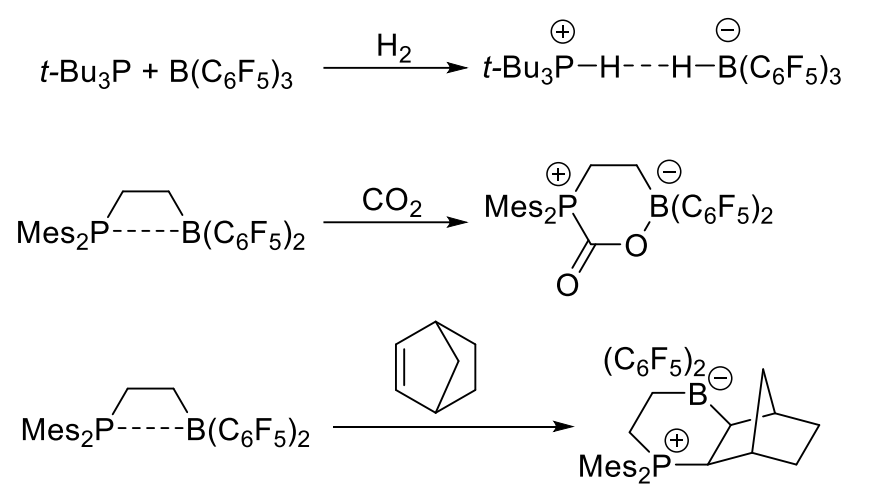

Scheme 1.5 Selected FLP complexes formed with small molecules. ${ }^{7-8,36-38}$

Small molecules can work as bridges to link up phosphorous and boron centres in polymer chains. The binding between small molecules and FLPs has been proved to be a dynamic process and temperature dependent in many cases. FLPs show several real-life applications due to their interesting reactions with small molecules; these mainly fit into three categories: metal-free hydrogenations, the capture of gas molecules, and catalysts for polymerisations.

\subsubsection{Metal-free Hydrogenations}

As a part of the initial findings arising from the discovery of FLPs, dihydrogen cleavage garners particular attention because it is a reaction that could previously only be achieved by d- or f-block transition metals, and indeed only very rare examples of non-metallic systems exist. ${ }^{7-8,10,32,39}$ Hence FLPs provided a possibility of developing non-metallic hydrogenation catalysts. ${ }^{7-8,32}$ There has been extensive discussion in the literature of the factors that may affect the efficiency of dihydrogen cleavage by FLP, which can be summarised as the Lewis acidity, Lewis basicity and product stabilisation. ${ }^{40}$ Stronger Lewis acids and bases can provide more reactive and productive reaction centres. Product stabilisation, meanwhile, which mainly depends on the ionic interactions inside the zwitterionic product, affects the thermodynamic equilibrium of the dihydrogen cleavage process. It has been found that all these factors 
can be tuned by modifying the molecular structures of Lewis acids and bases.

Due to the heterolytic nature of dihydrogen cleavage by FLPs, i.e. where the dihydrogen bonding is broken by the transfer both electrons to one of the hydrogen nuclei to give a proton and a hydride, the hydrogenation shows particularly high success with the molecule containing polar unsaturated bonds. Provided suitable Lewis acids and bases are carefully selected, taking into account the appropriate strength and steric hindrance to meet the energy requirements for various substrate molecules, FLPs have shown great versatility in the hydrogenation of different classes of compounds. ${ }^{40}$ The chemicals that could be hydrogenated by FLPs/ $\mathrm{H}_{2}$ includes imines, silyl enol ethers, enamines, etc. as shown below in Scheme 1.6.<smiles>[R]N=C([R])[R]</smiles><smiles></smiles><smiles>[R]C([R])=C([R])N([R])[R]</smiles>

Scheme 1.6 Selected examples of FLP hydrogenation substrates. Reductions of imine (top), silyl enol ether (middle), and enamine (bottom).

In the case of imines, the rate of conversion was found largely dependent on the electronic environment of the substrate compound, with the hydrogenation proceeding much faster when the imine is more electron rich. This phenomenon is consistent with the proposed reaction mechanism (Scheme 1.7 (A)) in which the imine nitrogen is protonated first by the phosphonium moiety of the FLP/ $\mathrm{H}_{2}$ adduct followed by a hydride insertion from the borate moiety. The hydride insertion in the proposed 
reaction mechanism is confirmed by the hydrogenation of an imine $\mathrm{MesN}=\mathrm{C}(\mathrm{Me})^{t} \mathrm{Bu}$, where the product obtained is not the reduced amine but a salt, $\left[\mathrm{MesNH}=\mathrm{C}(\mathrm{Me})^{t} \mathrm{Bu}\right]^{+}\left[\mathrm{HB}\left(\mathrm{C}_{6} \mathrm{~F}_{5}\right)_{3}\right]^{-}$because the iminium carbon is sterically hindered. The substituent attached on the nitrogen atom needs to be sufficiently sterically hindered to prevent the reduced product binding with the Lewis acid, which will terminate the hydrogenation process. The catalytic system could be modified to give better performance and increase the substrate scope. For example, in the case of hydrogenation of less sterically hindered imines, BCF could be added as a protection group to prevent the substrate compound binding with the boron inside the catalyst to deactivate it. A similar strategy has also been applied to the reduction of nitriles. Also, sometimes, a Lewis base is not necessary if the imine itself is sufficiently sterically hindered to form a dihydrogen active FLP with an externally added Lewis acid, ${ }^{41}$ as shown in Scheme 1.7 (B).

(A)

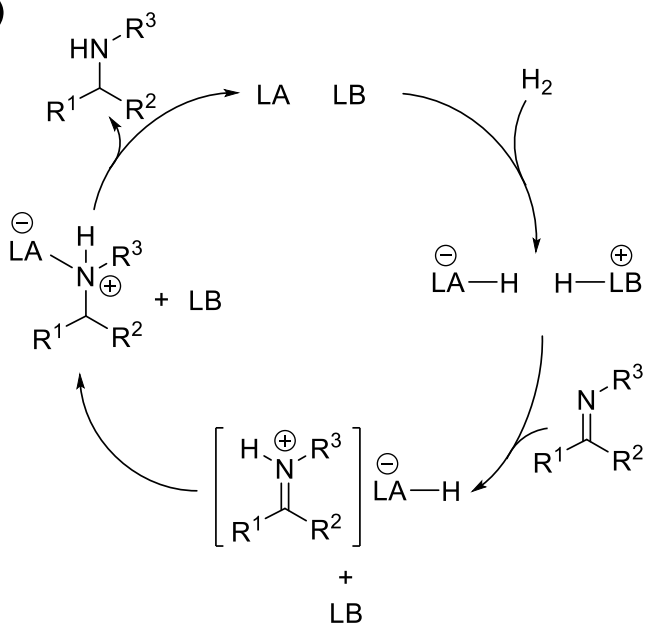

(B)

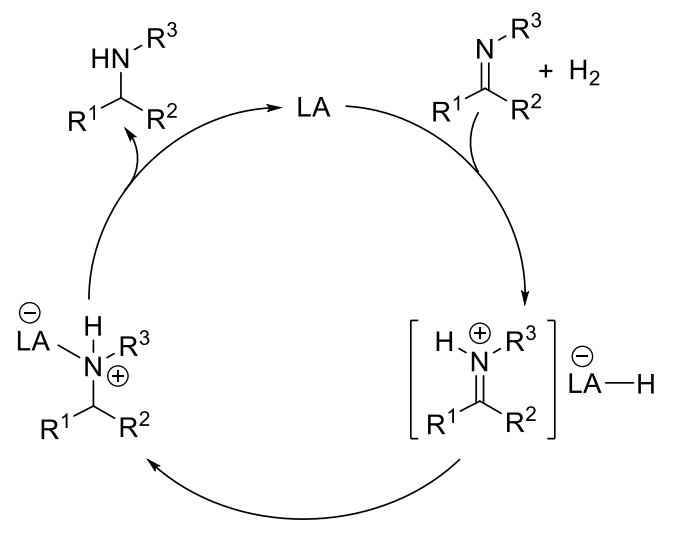

Scheme 1.7 Mechanistic representation of FLP-catalysed hydrogenation of imines. (A) Hydrogenation by LA/LB pair. (B) Self-catalysed reduction of imine as a base partner of an added LA.

Apart from imines, silyl enol ethers are another class of compounds that can be reduced by FLP hydrogenation (Scheme 1.6).$^{42}$ Like imine hydrogenation, the steric hindrance around the oxygen of a silyl enol ether substrate is also necessary to keep the Lewis 
acid active. The reduction of a silyl enol ether is also affected by the electronic status of the compound, and electron-rich silyl enol ether has also been proved to be able to self-reduce by acting as a Lewis base to pair with an external Lewis acid. Enamine is also a class of widely-studied compounds for FLP hydrogenation, which can convert efficiently into its derivative amines with mild reaction conditions. ${ }^{43}$ Recently, Ashley et al. also showed an ether solvent incorporated BCF hydrogenation reaction. ${ }^{44-}$ ${ }^{45}$ The ether molecule such as THF can readily work as the Lewis base to partner with BCF. The resultant LPA can achieve dihydrogen cleavage and hydrogenate not only imines, but also alkenes and some other heterocyclic compounds. Stephan et al. ${ }^{46}$ and Soós et al. ${ }^{47}$ also each independently investigated ether-assisted BCF hydrogenations, and successfully reduced ketones and aldehydes.

\subsubsection{Carbon Dioxide Capture}

While a variety of gaseous substrates have been shown to be activated by FLP chemistry, carbon dioxide continues to be particularly heavily investigated. The capture of this gas is inherently important as it provides a potential solution for global warming. In 2009, a FLP made of ${ }^{t} \mathrm{Bu}_{3} \mathrm{P}$ and $\mathrm{BCF}$ was found to be able to capture $\mathrm{CO}_{2}$ effectively (Figure 1.4). ${ }^{28}$ Although it has been shown that $\mathrm{CO}_{2}$ escapes from the FLPs at higher temperature, it could be recaptured immediately when the temperature was reduced. ${ }^{28}$ Following this, more examples of FLP-mediated $\mathrm{CO}_{2}$ activation have been reported. It is known that the stability of the FLP-CO $\mathrm{CO}_{2}$ adduct varies significantly with the structure of FLPs, as is revealed by very different $\mathrm{CO}_{2}$ capture and escape temperatures. One of the related reports involved using a series of boron Lewis acid $\mathrm{RB}\left(\mathrm{C}_{6} \mathrm{~F}_{5}\right)_{2}$ with ${ }^{i} \operatorname{Pr}_{3} \mathrm{P}$ (Figure 1.4) ${ }^{38}$ When the $\mathrm{R}$ group is more electron withdrawing, like chlorine, phenyl or 2,3,5,6-tetrafluorophenyl, the resultant $\mathrm{FLP}-\mathrm{CO}_{2}$ adduct tends to be more stable because of the enhanced Lewis acidity in the FLP. In contrast, when the $\mathrm{R}$ group is an alkyl group, the FLP- $\mathrm{CO}_{2}$ is always much less stable, and 
decomposition occurs at very low temperatures. Substituents on phosphine also give a similar effect in many examples, where the moieties that enhance the Lewis basicity of phosphine can dramatically decrease the $\mathrm{CO}_{2}$ capture temperature. The structure of a FLP also has a dramatic influence on the stability of the $\mathrm{FLP}-\mathrm{CO}_{2}$ adduct. One example is the $\mathrm{FLP}^{t} \mathrm{Bu}_{2} \mathrm{PCH}_{2} \mathrm{~B}\left(\mathrm{C}_{6} \mathrm{~F}_{5}\right)_{2}{ }^{48}$ Even though this molecule can undergo selfdeactivation quickly, it shows remarkable reactivity towards $\mathrm{CO}_{2}$. X-ray analysis has shown that the resultant adduct product is a near planar five-membered ring; a structure that enhances the overall stability and makes the product stable, even at temperatures in excess of $100{ }^{\circ} \mathrm{C}$ under a vacuum.

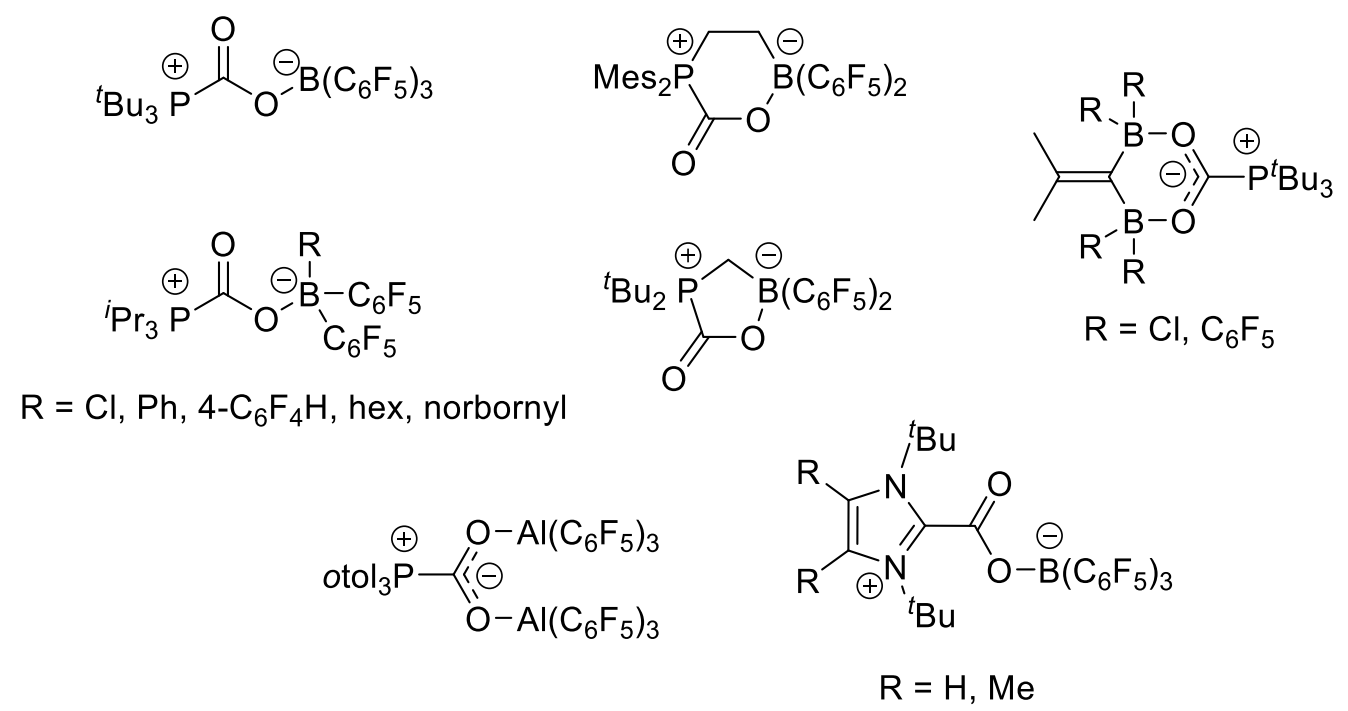

Figure 1.4 Selected examples of product of $\mathrm{CO}_{2}$ activation by FLPs. ${ }^{28,38,48-52}$

The number of Lewis centres that have been shown to activate $\mathrm{CO}_{2}$ as increased significantly from just the boron/phosphine-based FLPs originally reported, to now include aluminium, ${ }^{50}$ zirconium, ${ }^{53}$ as the Lewis acid, and carbenes, ${ }^{52}$ nitrogen, ${ }^{54}$ as the Lewis base. Some special designs of Lewis acid have also been reported in the literature, such a bi-functionalised boron or aluminium Lewis acid, in which both Lewis acid centres can bind with both of the oxygen atoms in activated $\mathrm{CO}_{2} \cdot{ }^{51}$ Analyses by NMR spectroscopy, X-ray crystallography and IR spectroscopy showed that such a 
system exhibits enhanced activation on the carbon in $\mathrm{CO}_{2}$. This provides valuable insights to inform the process of designing more active $\mathrm{FLP}-\mathrm{CO}_{2}$ capture systems. Also, a recently disclosed borane catalyst incorporated a $\mathrm{FLP}-\mathrm{CO}_{2}$ capturing system which successfully converted the captured $\mathrm{CO}_{2}$ gas into a usable $\mathrm{C}_{1}$ feedstock. ${ }^{55}$ This example illustrates the potential future work and achievable real applications of this field.

\subsubsection{FLPs as Catalysts for Polymerisations}

As an extension of small molecule activation, many researchers have noticed that FLPs could be used for polymerising olefin monomers because the binding between activated carbon and the Lewis acid compound is reversible. Indeed, even before the discovery that FLPs had the ability to catalyse polymerisations, and as far back as 1960 , there were examples of the use of conventional Lewis pairs (CLPs) as catalysts to synthesise polymers. ${ }^{56-57}$ The related work did not attract much attention at that time, however, since the polymerisation is slow and uncontrolled. ${ }^{57}$ In 2010 , Chen et al. reported their seminal work detailing the use of CLPs and FLPs as catalysts for polymerisation. ${ }^{58}$ Since then, FLPs have been found to show a good ability to polymerise many commercially available polar conjugated olefin monomers quickly (full conversion in several minutes even at very low temperatures, e.g. $-78^{\circ} \mathrm{C}$ ), with

good control of molecular weight, dispersity and polymer chain architectures. ${ }^{56-58}$ Nowadays in the literature, researchers are more likely to combine both CLP and FLP polymerisations into a bigger field called Lewis pair Polymerisation (LPP) because they are very similar in the reaction mechanism and sometimes show a grey area in between. The reaction mechanism of LPP was shown below in Scheme 1.8. ${ }^{56-57}$ 


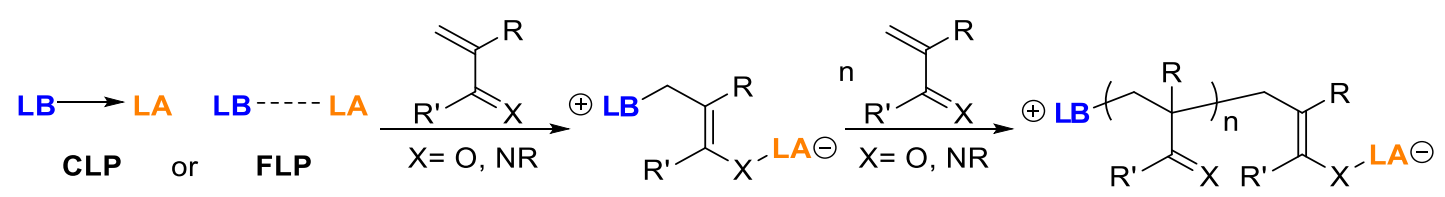

Scheme 1.8 Lewis Pair Polymerisation of a polar conjugated olefin monomer. ${ }^{56-57}$

Although there are similarities between LPP and other zwitterionic polymerisations, they should not be entirely equated. One of the biggest differences is that both the Lewis acid and Lewis base used in LPP are incorporated in chain initiation, propagation, transfer and terminations, in contrast to zwitterionic polymerisations where only one of the charged species is involved in those processes. ${ }^{56-57}$

The rate and control within LPP greatly depends on the stability of the active zwitterionic complex formed between the Lewis pair and monomer. ${ }^{56}$ Researchers have tended to focus more on $\mathrm{E}\left(\mathrm{C}_{6} \mathrm{~F}_{5}\right)_{3}(\mathrm{E}=\mathrm{B}, \mathrm{Al}), \mathrm{R}_{3} \mathrm{Si}^{+}, \mathrm{AlR}_{3}$, etc. for Lewis acids for use in LPP, since these show excellent catalytic effects on the rate of polymerisation. ${ }^{56-}$ 57 The Lewis bases used, meanwhile, include phosphines, amines, N-heterocyclic carbene (NHC), organophosphine superbase, N-heterocyclic olefin (NHO), etc. ${ }^{56-57}$ By pairing different Lewis acids and bases it is easy to tune the reactivity of the resultant Lewis pairs, thereby affecting the performance of polymerisations. For example, $\mathrm{Al}\left(\mathrm{C}_{6} \mathrm{~F}_{5}\right)_{3} / \mathrm{NHC}$ can give an extra fast rate of polymerisation with relatively low control of dispersity, ${ }^{57-58}$ whereas $\mathrm{MeAl}(\mathrm{BHT})_{2} / \mathrm{NHO}$ gives slower polymerisations but the reaction has almost perfect control of dispersity. ${ }^{57,59}$ Besides vinyl monomers, Lewis pairs can also polymerise lactides and other cyclic ester monomers via ring-opening polymerisation. ${ }^{57,60}$ 


\subsection{Dynamic Crosslinked Polymeric Networks and Self-Healing Materials}

Conventional thermoset polymeric materials have good mechanical properties due to their permanent crosslinks between polymer chains. They have found a wide range of applications such as making high impact-strength materials. The crosslinking reaction that occurs during the production processes is irreversible, however. Therefore, it is impossible to reshape the materials after production, and repairing the damaged at the molecular level is also very difficult. Both situations make these materials difficult to recycle. Many different attempts have been made to "heal" damaged thermoset material. For instance, self-healing by introducing microcapsules or microvasculature into the materials, which contain monomers and catalysts that can be released during

cracking. ${ }^{61-68}$ Although such systems have achieved some successful self-healing, the number of times of the healing are seriously limited due to the reduction and degradation of healing agents after each cracking event. Also, the self-healing relies on the embedded healing agents rather than the inherent chemical structure of these materials, so they possess no reshaping ability. In order to improve the performance of thermoset materials at a molecular level, recent research has incorporated reversible bonds into the crosslinks of these materials. ${ }^{69}$ Such reversible bonds include not only covalent bonds, but also many different types of non-covalent supramolecular interactions. A material with these dynamic crosslinks can be reshaped after processing, and can self-heal, while being able to respond to certain stimuli. The following sections of this chapter will introduce these smart materials.

\subsubsection{Covalent Adaptable Networks}

Dynamic covalently crosslinked polymers, also known as covalent adaptable networks $(\mathrm{CAN})$, are thermoset polymeric networks with reversible covalent crosslinks that can 
respond to an applied stimulus. ${ }^{69}$ Since the crosslinks can be broken and reformed under certain conditions, rearrangement of polymer chain connections can be achieved, allowing the material to reshape or "self-heal". Various types of reversible covalent bonded groups have been applied as crosslinking for CANs, including carbon-carbon groups based on Diels-alder ${ }^{70}$ or $[2+2]$ cycloaddition, ${ }^{71}$ disulphide/thiol, ${ }^{72-74}$ siloxane, ${ }^{75}$ boronic esters, ${ }^{76-79}$ boroxine,${ }^{80}$ etc. Depending on the way in which they respond to external stimuli, the CANs are also classified into two categories: thermally or photo-triggered systems. Selected examples of these crosslinking are shown below in Scheme 1.9.

The self-healing normally involves several steps: firstly, after cracking, the fractured interfaces are brought into close contact with each other. Then, either triggered by heat or radiation, the crosslinks become dynamic and exchange with each other between the cracked interfaces. This ultimately leads to healing at molecular level, with significant restoration of the material's original mechanical properties. While, in some cases, however, the self-healing efficiency is not ideal due to limited crosslinking exchanges as a result of poor chain mobility, in other cases, such as vitrimers, there is full recovery of mechanical properties after healing, mainly due to their high fluidity in their triggered state. ${ }^{78}$ 

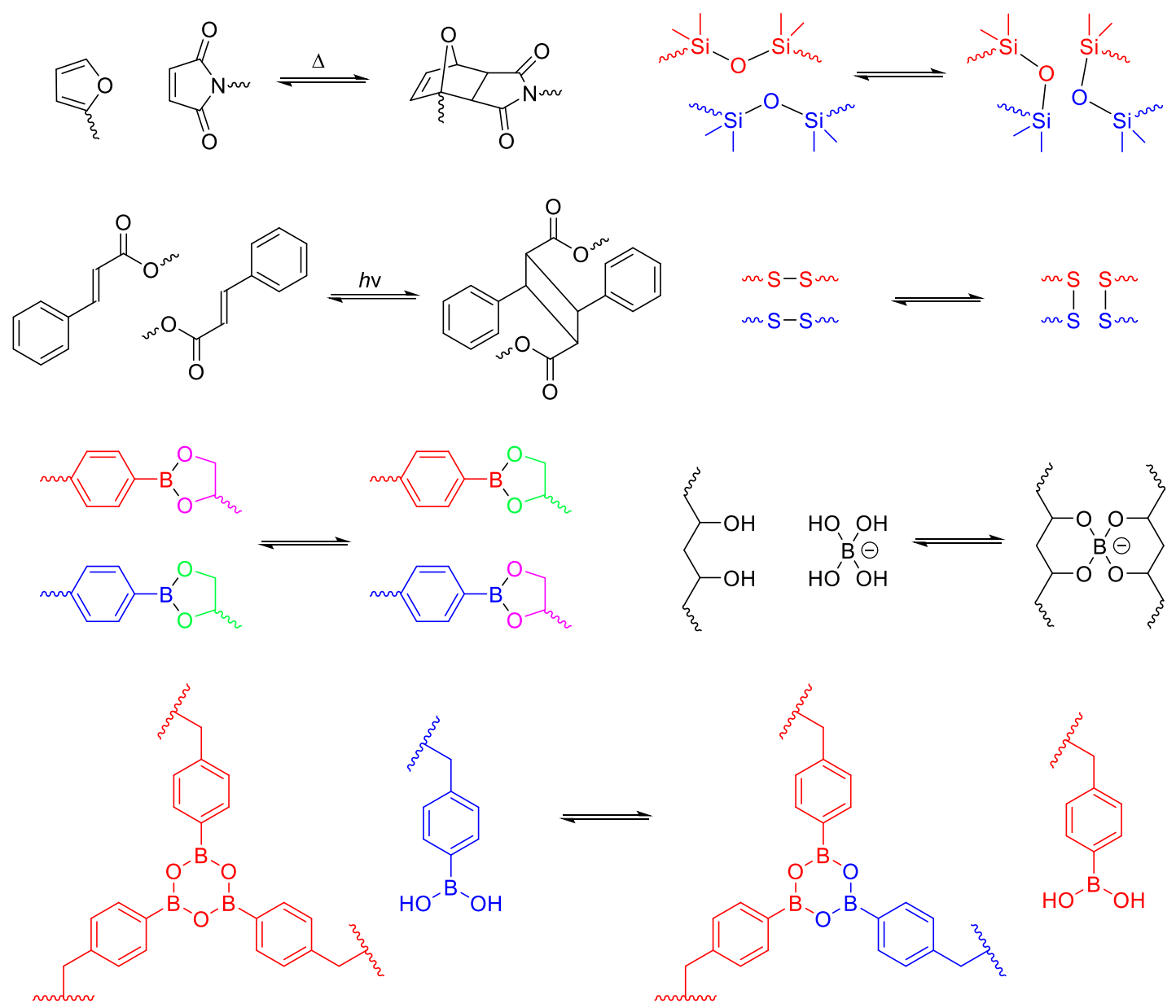

Scheme 1.9 Schematic representations of selected examples of covalent dynamic crosslinks. ${ }^{70-72,75,77-78,80}$ Wavy bonds represent polymer backbones.

\subsubsection{Supramolecular Chemistry Based Polymeric Networks}

Ever since the introduction of supramolecular chemistry, significant research interest has focused on implementing it into smart polymers. A supramolecular polymer is defined as a polymer in which repeating units are connected by supramolecular noncovalent interactions, ${ }^{81}$ such as ionic crosslinks, ${ }^{82-86}$ hydrogen bonding, ${ }^{81,87} \pi-\pi$ interactions, ${ }^{88-89}$ hydrophobic forces, metal coordination, etc. ${ }^{90}$ Similar to CANs, supramolecular polymers are considered attractive on account of their self-healing activity, which arises from the ability of their chains to be opened and re-joined dynamically without the need for any embedded material, such as potentially toxic 
catalysts. ${ }^{91}$ In the following sections, different types of supramolecular crosslinks will be introduced, together with some selected examples.

\subsubsection{1 lonic Crosslinking}

Ionomers are a class of polymer which contain a maximum of $20 \%$ ionic species. ${ }^{84}$ Ionomers have been widely studied for their unique chemical structure and mechanical properties including many successful applications in industry, e.g. DuPont Nucrel ${ }^{\circledR}$ series. ${ }^{84}$ The crosslinking part of an ionomer is normally referred to as a cluster (Figure 1.5 (A)) ${ }^{84,92-93}$ A cluster is a complex of several multiplets (Figure 1.5 (A)), which is the most basic aggregation units of polymer chains. ${ }^{84,93}$ The aggregation in multiplets is caused by the electrostatic forces between ionic species and the physical entanglements between polymer chains. Consequently, the crosslinking of an ionomer material is usually a result of a combination of physical entanglements and electrostatic forces between ions. ${ }^{84,93}$ When the concentration of ionic contents is above a threshold value, the clusters within the ionomer will be abundant enough to generate phases of separation. ${ }^{84,94}$ At this stage, the ionomer could be regarded as similar to thermoset polymers, and its clusters will work in a similar way to covalent crosslinks, thus enabling the ionomer to exhibit physical performance as good as other conventional polymer materials. ${ }^{84}$ 

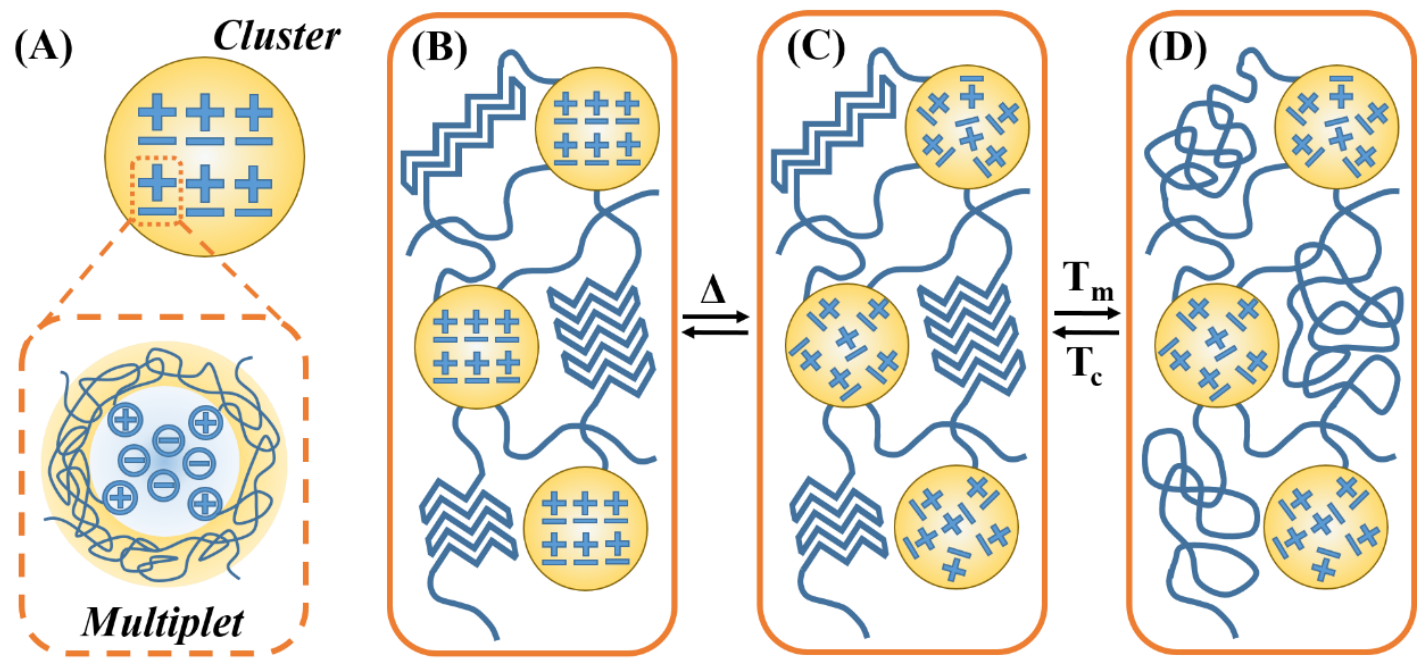

Figure 1.5 Figure to represent the structure of (A) cluster and multiplet; (B) ionomer with ordered cluster and crystallinity; (C) ionomer with disordered cluster and crystallinity and (D) melted ionomer. ${ }^{84,92}$

Due to the special internal structure of ionomers, their self-healing usually requires external stimuli, such as heating, in order to enhance the chain mobility by reducing the physical entanglements between the polymer chains inside clusters. Taking poly(ethylene-co-methylacrylic acid) ionomer as an example, from the research by Tadano et al., it could be shown that when the temperature goes above the order-todisorder transition temperature, the ordered internal structure inside clusters will be lost and the physical entanglement will decrease dramatically (Figure 1.5 (B)-(C)). ${ }^{92}$ If the temperature continues to increase, the crystallinity of macroscopic polymers will break down (Figure 1.5 (D)). ${ }^{92}$ The self-healing of such a material, therefore, normally consists of the following steps: Firstly when the ionomer is damaged seriously (e.g. penetration by a bullet), the impact energy on the fractured surface will be dissipated as heat, hence melting the local structures. ${ }^{84,86,93,95-96}$ The newly disordered clusters allow chain exchange to happen between them; ${ }^{93}$ Then the crystallinity of material will be recovered quickly upon cooling; ${ }^{84,86,93}$ Lastly the ordered structure inside the clusters will be reformed but on a much slower timescale. ${ }^{84,86,93}$ This slow recovery of the cluster structure often results in the incomplete healing of ionomers. Nonetheless, 
the self-healing of ionomers is still thought to one of the most robust systems as there are very few other systems that can self-heal a serious puncture which leaves a hole with a diameter of several millimetres. In recent years there are also many examples of new ionomers which can self-heal autonomously without heating. ${ }^{85}$ New systems continue to be explored.

\subsubsection{2 $\pi-\pi$ Stacking Interaction Crosslinking}

The ionomers introduced above are a relatively unique example of supramolecular crosslinked systems, since their ionic interactions are relatively strong compared to other supramolecular forces. The exploration of dynamic crosslinks using much weaker interactions is also popular. $\pi-\pi$ stacking interactions constitute weak intermolecular forces which are generated between overlapped aromatic macrocycles. ${ }^{97}$ Even though such interactions are intrinsically very small, within a complete polymeric material they will exist in very large quantities. Hence, the overall force will be strong enough to hold the polymer material together intact. Most of the preliminary work regarding $\pi$-stacking incorporated into polymers has been to incorporate macrocycles into the backbone of a polymer chain. ${ }^{98-100}$ The resultant polymer is then able to self-assembly by the stacking interaction between aromatic repeating units.

In 2010, Colquhoun et al. developed a polymeric organogel by using two different polymeric macro-gelators (Figure 1.6). ${ }^{88}$ One of these gelators is a copolymer containing naphthalenediimide as the host sites, the other one is a telechelic polymer, both ends of which are capped with pyrene moieties as the guests. From their previous work, the authors had proved the good binding abilities between the naphthalenediimide and pyrene groups, ${ }^{101}$ and the association constant between the host and guest molecules was determined to be up to $11000 \mathrm{M}^{-1} \cdot{ }^{101}$ To make the gel, 
the two copolymer solutions were mixed together with 1:1 equivalents of diimide and pyrene functionalities. Upon mixing, an obvious colour change from colourless to deep-red was observed, which is an indicator of host-guest binding. This supramolecular binding was also found to be able to retain in solid state by casting a film containing both copolymer gelators. The rheological data shows that the material created comprised a crosslinked network rather than an extended single chain polymer. The self-healing ability was tested by tensile stress. The cast polymer film was stressed to break, then healed at $110{ }^{\circ} \mathrm{C}$. The results showed that the material was able to attain a 95\% self-healing efficiency after $240 \mathrm{~min}$ at this temperature. Repeated healing also showed no obvious decrease in physical properties, indicating that the system is indeed an intrinsic self-healing material. Following this report, Rowan et al. were able to reinforce the mechanical properties of this polymer by incorporating cellulose nanocrystals in order to create a self-healable nanocomposite. ${ }^{89}$

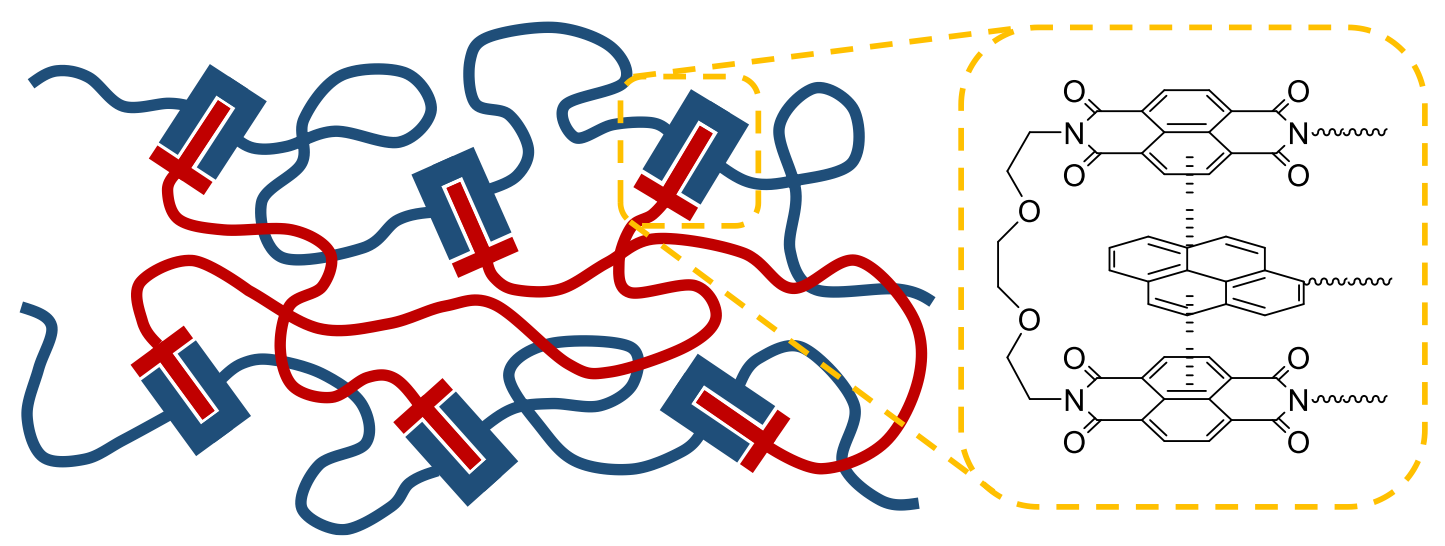

Figure 1.6 $\pi$-stacking interaction based crosslinked organogel by Colquhoun et al. ${ }^{88}$

\subsubsection{Hydrogen Bond Crosslinking}

Hydrogen bonding is widely used in supramolecular polymers because they are convenient to prepare, directional, reversible and colourless. ${ }^{91}$ The first example of a hydrogen-bonded supramolecular polymer was introduced by Lehn et al. in 1990, based on two bi-functionalised oligomer units. The linkages between these oligomers 
were achieved by three hydrogen bonds. After that, Meijer et al. reported their quadruple hydrogen bonded polymers in 1997, which became one of the most wellknown hydrogen-bonded supramolecular polymer, shown below in Figure 1.7. ${ }^{81,87}$

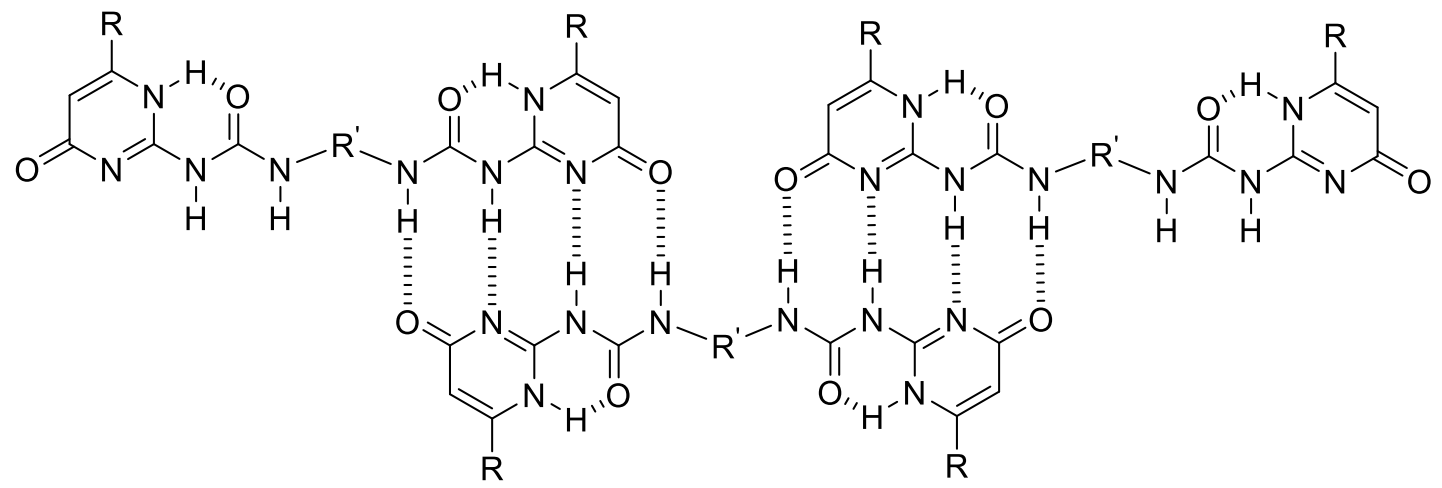

Figure 1.7 Quadruple hydrogen bonding crosslinked polymer by Meijer et al. ${ }^{81,87}$

As shown above, Meijer et al.'s work started from preparing linear single chain polymers by using a telechelic oligomer with both ends functionalised with ureidopyrimidinone (UPy) groups. These UPy groups were found to dimerise readily intermolecularly via quadruple hydrogen bonds, and the dimerisation constant was found to be greater than $10^{6} \mathrm{M}^{-1}$ in chloroform at room temperature. ${ }^{87}$ One important reason for using the UPy group as a crosslinking moiety is to avoid any uncontrolled multi-directional gelation, which had frequently been found in previous works. Although the associations between UPy groups are very strong, the dimers formed are still reversible, which makes self-healing and reprocessing possible. This is confirmed by adding a capping agent which has only one UPy group in it. A dramatic decrease in the viscosity of the polymer solution confirms that an exchange reaction has occurred between the monomer and capping agent, which is a result of the reduction in the molecular weight. ${ }^{87}$ Furthermore, the molecular weight of such a polymer was found to be dependent on the solution concentration of the monomer, with the polymer being able to achieve an estimated molecular weight of $5 \times 10^{5} \mathrm{~g} / \mathrm{mol}$ when the concentration of monomer is $40 \mathrm{mM} \cdot{ }^{87}$ Meijer et al. also extended their work to prepare a polymer gel using a tri-functionalised UPy oligomer. ${ }^{87}$ By comparing this with a similar 
polymer gel with irreversible crosslinking, mechanical testing showed the dynamic crosslinked polymer gel to have a higher mechanical performance overall. ${ }^{87}$ This was a result of the denser polymer network, which is achieved by crosslinking exchange. This means the hydrogen bonding interactions convert the material from a kineticcontrolled system to a thermodynamic controlled system. ${ }^{87}$ The self-healing activity of this polymer was discovered later by Tessa ten Cate, who found that cut pieces of this kind of material could be re-joined by pressing the pieces together for several minutes. ${ }^{91}$ This is achieved by the reformation and exchange of dimer junctions between fractured interfaces. This kind of material could also be healed by an even more robust method. The whole material could be melted down first and then cooled into moulds. Since hydrogen bonds are sensitive to temperature and break upon heating, ${ }^{81}$ the solid polymer gel is able to convert back to a liquid monomer at high temperature and the solid polymer gel could be simply re-obtained once the liquid monomers was allowed to cool back to room temperature. ${ }^{81}$ Another advantage of this material is its aforementioned concentration-dependent viscosity/molecular weight, which allows the material to be stored in solvent at low concentration, and sprayed out when a surface is required. ${ }^{81}$ After this work more hydrogen-bonded polymer systems have been developed and several reports continue to be disclosed to this day.

\subsubsection{Van der Waals Forces based Crosslinking}

As mentioned above, weak supramolecular interactions such as hydrogen bonding have been widely exploited in materials chemistry. Even though these interactions are relatively weak, the large number of them present within a polymeric structure can result in a polymer network with increased or tuneable strength. Relative to hydrogen bonding, Van der Waals forces are normally thought to be even weaker, possessing an energy approximately 10-20 times smaller than hydrogen bonding. ${ }^{102}$ Due to this, it is a significant challenge to use this type of interaction as a crosslinking mechanism for 
polymer networks. Very recently Urban et al. successfully synthesised and reported the first example of a self-healing polymeric material that uses Van der Waals forces to crosslink polymer chains (Figure 1.8). ${ }^{103}$ These materials were made by copolymerisation of methyl methacrylate (MMA) and n-butyl acrylate (nBA) via atom transfer radical polymerisation (ATRP) with a broad range of compositional ratios. Interestingly, the authors noticed that some of the copolymers obtained where the MMA/nBA molar ratios ranged between $45 / 55$ to $50 / 50$ displayed self-healing properties. This finding encouraged them to explore the mechanism behind the selfhealing of this interesting material further. Through molecular dynamic (MD) simulation, it was found that the sequence of randomly copolymerised monomers in the polymer chain plays an important role in the self-healing behaviour, effectively turning the copolymer chains into "lock and key" components. When the "key" part from one polymer chain matches the "lock" part from another chain in energy, a crosslinking between these two chains will be generated. Such a crosslinking event is mainly possible by Van der Waals forces, and the strength of these interactions depends to a large extent on the monomer sequence in the "lock and key" region. By considering a five-repeating-unit as a group, the cohesive energy between polymer chains was simulated to be between $313.6 \mathrm{~kJ} / \mathrm{mol}$ to $258.2 \mathrm{~kJ} / \mathrm{mol}$, which are surprisingly high values for Van der Waals forces. Such a high interaction strength enables the material to display mechanical properties as good as those of covalently crosslinked materials, whose C-C bond energy is usually about $350 \mathrm{~kJ} / \mathrm{mol}$. Upon breaking and re-joining the "lock and key", the material was shown to heal intrinsically. When the overall molar ratio of MMA increases, the interfacial fluidity will decrease, which disfavours the formation of inter-chain crosslinking. Also, if more nBA is incorporated, the quantities of Van der Waals force will be limited. This is why there is a the very narrow window of $\mathrm{MMA} / \mathrm{nBA}$ molar ratio displaying this self-healing behaviour. Although this is a clear limitation, this system is still very robust and provides a very novel approach to designing self-healing materials. One key benefit of 
this system is that no special functional groups need to be synthesised and incorporated, which is very valuable for synthetic and economic considerations.

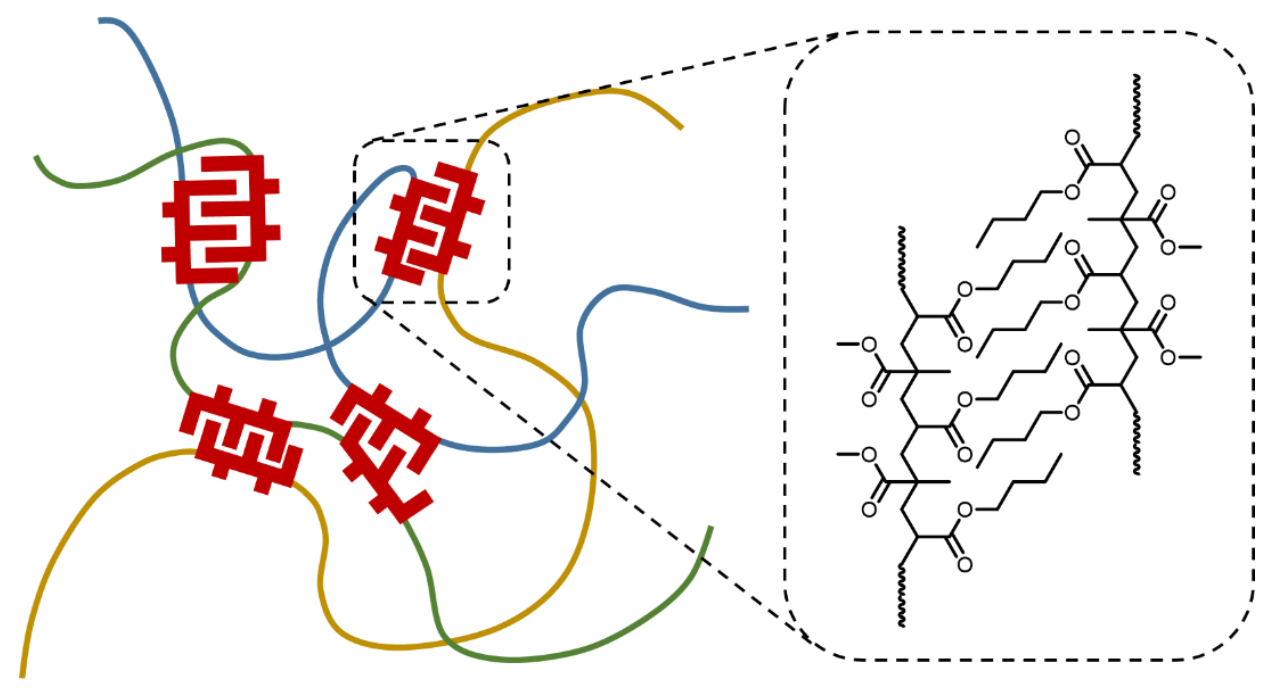

Figure 1.8 "Lock and Key" Van der Waals force crosslinked self-healing polymers by Urban et al. ${ }^{103}$

\subsubsection{Conventional Lewis Pair Based Crosslinking}

Incorporation of conventional Lewis pair adducts into polymers has been studied for approximately two decades. The Lewis bases and acids in the CLP-crosslinked polymers are more limited to main group compounds and they can be distinguished in this way from other coordination interactions like metal-metal and metal-ligands interactions. The Lewis acids and bases used are normally boron and nitrogen derivatives, which will form relative stable dative covalent bonds with each other. To the best of our knowledge, the first example of a supramolecular polymer that was generated via a Lewis pair interaction was reported by Wagner et al. in $1998 .{ }^{104-105} \mathrm{In}$ that case, two small molecules were generated each bearing boron and nitrogen moieties, and a self-assembled linear macromolecular structure was produced upon mixing (Scheme 1.10 (A)). The dynamic nature of the crosslinking was found by mixing the two monomers with $p$-methylpyridine. A competitive binding between the 
two pyridine derivatives was observed, with the one used as a monomer, 4,4'bipyridine, showing a stronger tendency to bind with the boron. From their research, Wagner et al. noted that, in the solid state, the polymer product shows a good thermal stability, however when it is dissolved at $85{ }^{\circ} \mathrm{C}$, the polymer can be fully depolymerised back to monomers due to the thermal cleavage of the $\mathrm{B}-\mathrm{N}$ dative covalent bonds. Even though this polymer system shows exciting thermally triggered reprocessing and self-healing ability, the authors chose to pursue instead the interesting electrochemical properties of this material, and research into its unique polymeric behaviour has not yet been reported.

(A)
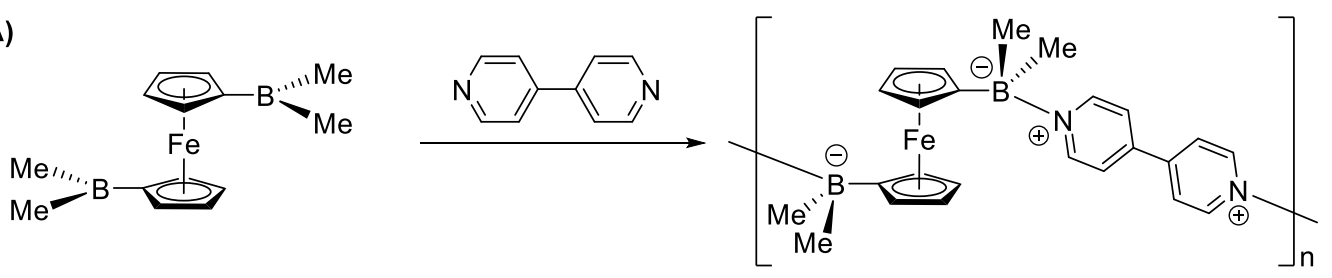

(B)
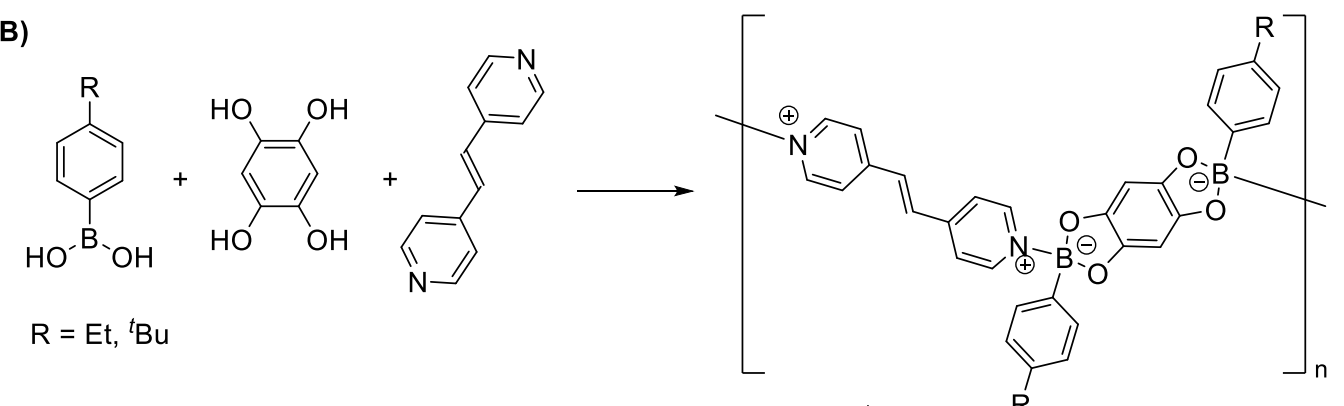

(C)

$\mathrm{R}=\mathrm{Et},{ }^{\mathrm{t}} \mathrm{Bu}$

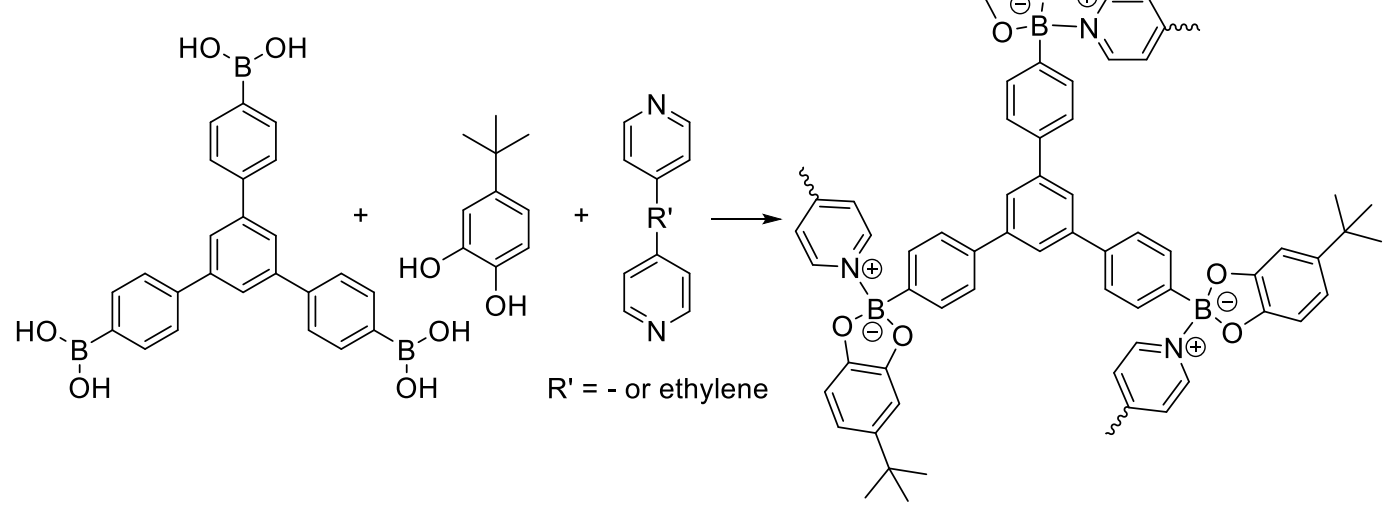

Scheme 1.10 Schematic representation of CLP supramolecular polymer made by (A) Wagner et al. ${ }^{104-105}$ and (B)-(C) Severin et al. ${ }^{106-107}$ 
In 2007, Severin et al. published their work about a Lewis pair-containing polymer using a catechol borinate ester as the Lewis acid and 1,2-bis(4-pyridyl)ethylene as the Lewis base (Scheme 1.10 (B)). ${ }^{106}$ The polymer was synthesised by a one-pot threecomponent reaction, where the Lewis acid was synthesised in situ by 4-alkylphenyl boronic acid and 1,2,4,5-benzenetetrol. Both the Lewis acid and the Lewis base monomers are therefore bi-functionalised, and the resultant product obtained is a single chain linear polymer. From X-ray analysis, this polymer chain adopts a zig-zag conformation due to the strain around the catechol unit. By ${ }^{11} \mathrm{~B}$ NMR spectroscopy, this polymer was found to be unstable when dissolved in hot chloroform since a peak that corresponds to a free borinate ester was observed instead of a tetra-coordinated one. This observation proved that the interaction between boron and nitrogen is fully reversible and temperature responsive. After this work, Severin et al. developed their system and extended the project into the formation of an organogel (Scheme 1.10 (C)). ${ }^{107}$ A similar synthetic route was used, by which the polymer network was made by a one-pot, three-component reaction. This time, a tri-functionalised Lewis acid monomer was made using a tri-functionalised boronic acid and a mono-functionalised catechol. In this instance, instead of making single chain polymers, a polymer network was produced. The gel was characterised by X-ray crystallography and was shown to possess a two-dimensional layered structure with voids throughout the network. Similar to the earlier work, this polymer gel was found to be thermally responsive since the B-N dative bond was shown to break efficiently when a solution of the polymer was heated. At $90^{\circ} \mathrm{C}$, all B-N interactions were found to be broken and a fully swollen gel was depolymerised back to a monomer solution. Later, in 2014, Brook et al. also reported a polymer gel based on a borinate ester-amine dative bond, which possesses similarities to Severin's system. ${ }^{108}$ This time, Brook et al. first incorporated both the Lewis acid and the Lewis base into single chain polysiloxane before gelation. Upon mixing of two macro-gelators, a three-dimensional polymer gel was obtained. The dynamic nature of the crosslinking was tested by addition of a competitive base, 
n-butylamine. Rheological analysis also confirmed the thermal responsiveness of the Lewis pair crosslinking, which broke with elevated temperature.

Triarylborane based Lewis pair crosslinking have also been reported in the literature. In 2007, Jäkle et al. reported a polystyrene-based Lewis pair crosslinked organogel (Scheme 1.11 (A)). ${ }^{109}$ The phenyldithiophenylborane-capped ditelechlic copolymer was made through a post-polymerisation modification route, and when this was mixed with a small molecule, 4,4'-bipyridine, an extended single chain polymer was synthesised. The triarylborane used in this study had a much higher Lewis acidity compared to the borinate ester stated above, and hence it gave a very stable connection with the bipyridine group, even in solvent. Although the dynamic nature of such a polymer was not illustrated in detail in this work, recently, the same group published a thermal-responsive self-healing polymer based on a similar crosslinking system (Scheme 1.11 (B)). ${ }^{110}$ Rather than an end-functionalised copolymer chain, the same borane repeat unit was this time incorporated randomly into polystyrene chains. Also, both-end pyridine capped polysiloxane chains were used instead of small molecules as the Lewis base gelator. Not surprisingly, upon mixing of the two macro-gelators, a thermal responsive gel was obtained. The temperature dependence of the organogel formed was tested through a rheology temperature-ramp experiment and the modulus/viscosity vs. temperature curve clearly showed both the storage/loss moduli and the complex viscosity of the material decreasing with elevated temperature. The cross-over point where the loss modulus became higher than the storage modulus was determined to be $39.7^{\circ} \mathrm{C}$. Such a system not only successfully applied Lewis pair crosslinking into polymer networks, but also offered an insight into how mixed polymer backbones can be made into a consistent polymer gel. 
(A)

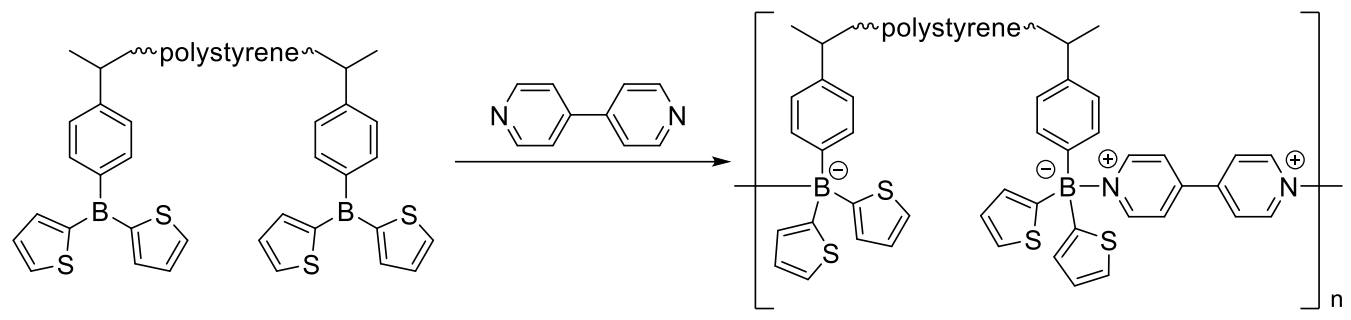

(B)

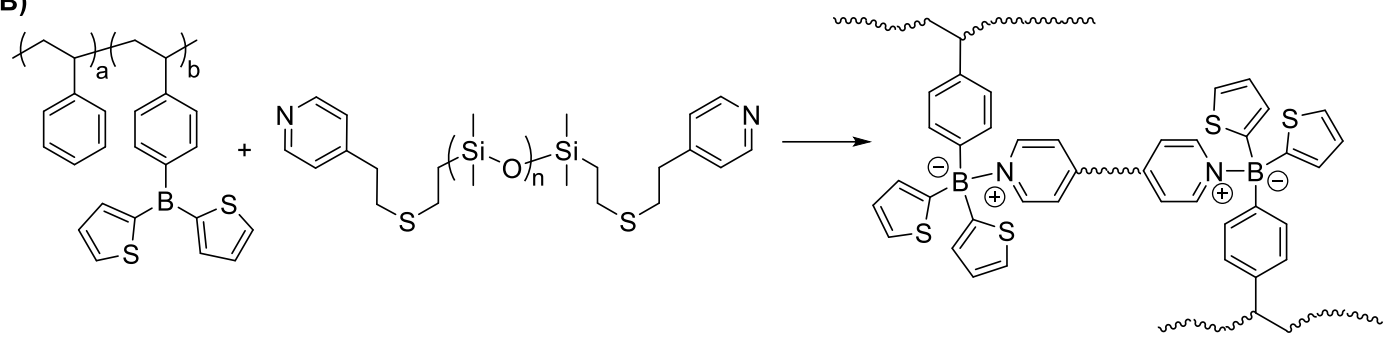

Scheme 1.11 Schematic representations of conventional Lewis pair crosslinked supramolecular polymers by Jäkle et al. ${ }^{109-110}$

\subsection{Aims of the Project}

As stated in previous sections, different dynamic crosslinking have been widely used in the design of responsive self-healing polymers. Based on different types of interactions, crosslinks lead to unique mechanical behaviours in the resultant polymer networks. To the date at which this project started, however, materials with crosslinking based on frustrated Lewis pairs were still an unexplored area. Although a semi-immobilised FLP polymer network was published by Thomas et al. during the course of this project, ${ }^{111}$ the polymer network in their work was based on a phosphine containing covalently crosslinked organogel, and the Lewis acid simply adheres onto the polymer network and does not participate in formation of crosslinks.

We were intrigued about the physical and chemical properties of a FLP crosslinked material, along with its potential for use in reaction catalysis. The overall aim of the project, therefore, is to synthesise a polymeric network using frustrated Lewis pairs as crosslinking moieties. Chapter Two focuses on the synthesis of our first generation 
polymeric-based frustrated Lewis pairs. The synthesis of individual Lewis acid (borane) and Lewis base (phosphine) moieties containing styryl monomers is introduced. Their copolymerisation with commercially available monomers, e.g. styrene or methyl methacrylate, is investigated. The characterisation of the resultant copolymers are also discussed. Thereafter, in Chapter Three, the gelation experiments of first generation polymeric FLPs macro-gelators is introduced, and the related characterisation is also presented. Lastly, our continuing work on developing nextgeneration polymeric FLPs is introduced in Chapter Four. Although this work is still ongoing, the chapter presents the process by which the monomers are synthesised and how these are used in the activation of small molecules.

\subsection{References}

[1] A Dictionary of Chemistry. 7th ed.; Oxford University Press: United Kingdom, 2016.

[2] Kauffman, G. B., J. Chem. Edu. 1988, 65 (1), 28.

[3] Housecroft, C. E.; Sharpe, A. G., Inorganic Chemistry. 3rd ed.; Pearson Education Limited: United Kingdom, 2008; p 183.

[4] Housecroft, C. E.; Sharpe, A. G., Inorganic Chemistry. 3rd ed.; Pearson Education Limited: United Kingdom, 2008; p 191.

[5] Brown, H. C.; Schlesinger, H. I.; Cardon, S. Z., J. Am. Chem. Soc. 1942, 64 (2), 325-329.

[6] Brown, H. C.; Kanner, B., J. Am. Chem. Soc. 1966, 88 (5), 986-992.

[7] Stephan, D. W., Dalton Trans. 2009, (17), 3129-3136.

[8] Stephan, D. W.; Erker, G., Angew. Chem. Int. Ed. 2010, 49 (1), 46-76.

[9] Welch, G. C.; Stephan, D. W., J. Am. Chem. Soc. 2007, 129 (7), 1880-1881. 
[10] Stephan, D. W.; Erker, G., Angew. Chem., Int. Ed. 2015, 54 (22), 6400-6441.

[11] Laurence, C.; Graton, J.; Gal, J.-F., J. Chem. Edu. 2011, 88 (12), 1651-1657.

[12] Sivaev, I. B.; Bregadze, V. I., Coordination Chemistry Reviews 2014, 270, 7588.

[13] Gutmann, V., Coordination Chemistry Reviews 1976, 18 (2), 225-255.

[14] Jensen, W. B., Chem. Rev. 1978, 78 (1), 1-22.

[15] Laurence, C.; Gal, J.-F., The Donor Number or SbCl5 Affinity Scale. In Lewis Basicity and Affinity Scales, John Wiley \& Sons, Ltd: 2009.

[16] Fawcett, W. R., J. Phys. Chem. 1993, 97 (37), 9540-9546.

[17] Hahn, S.; Miller, W. M.; Lichtenthaler, R. N.; Prausnitz, J. M., J. Solut. Chem. 1985, 14 (2), 129-137.

[18] Pearson, R. G., J. Am. Chem. Soc. 1963, 85 (22), 3533-3539.

[19] Laurence, C.; Gal, J.-F., The BF3 Affinity Scale. In Lewis Basicity and Affinity Scales, John Wiley \& Sons, Ltd: 2009.

[20] Allen, D. W.; Taylor, B. F., Journal of the Chemical Society, Dalton Transactions 1982, (1), 51-54.

[21] Kroshefsky, R. D.; Weiss, R.; Verkade, J. G., Inorg. Chem. 1979, 18 (2), 469472.

[22] Tassone, J. P.; Mawhinney, R. C.; Spivak, G. J., J. Organomet. Chem. 2015, $776,153-156$.

[23] Beckmann, U.; Süslüyan, D.; Kunz, P. C., Phosphorus, Sulfur, and Silicon and the Related Elements 2011, 186 (10), 2061-2070.

[24] Chevykalova, M. N.; Manzhukova, L. F.; Artemova, N. V.; Luzikov, Y. N.; Nifant"ev, I. E.; Nifant"ev, E. E., Russ. Chem. B. 2003, 52 (1), 78-84.

[25] Beckett, M. A.; Strickland, G. C.; Holland, J. R.; Sukumar Varma, K., Polymer 1996, 37 (20), 4629-4631.

[26] Beckett, M. A.; Brassington, D. S.; Coles, S. J.; Hursthouse, M. B., Inorg. Chem. Commun. 2000, 3 (10), 530-533. 
[27] Childs, R. F.; Mulholland, D. L.; Nixon, A., Canadian Journal of Chemistry 1982, $60(6), 801-808$.

[28] Mömming, C. M.; Otten, E.; Kehr, G.; Fröhlich, R.; Grimme, S.; Stephan, D. W.; Erker, G., Angew. Chem., Int. Ed. 2009, 48 (36), 6643-6646.

[29] Sajid, M.; Lawzer, A.; Dong, W.; Rosorius, C.; Sander, W.; Schirmer, B.; Grimme, S.; Daniliuc, C. G.; Kehr, G.; Erker, G., J. Am. Chem. Soc. 2013, 135 (49), 18567-18574.

[30] Cardenas, A. J. P.; Culotta, B. J.; Warren, T. H.; Grimme, S.; Stute, A.; Fröhlich, R.; Kehr, G.; Erker, G., Angew. Chem., Int. Ed. 2011, 50 (33), 7567-7571.

[31] Sajid, M.; Klose, A.; Birkmann, B.; Liang, L.; Schirmer, B.; Wiegand, T.; Eckert, H.; Lough, A. J.; Fröhlich, R.; Daniliuc, C. G.; Grimme, S.; Stephan, D. W.; Kehr, G.; Erker, G., Chem. Sci. 2013, 4 (1), 213-219.

[32] Stephan, D. W.; Erker, G., Frustrated Lewis Pair Mediated Hydrogenations. In Frustrated Lewis Pairs I: Uncovering and Understanding, Erker, G.; Stephan, D. W., Eds. Springer Berlin Heidelberg: Berlin, Heidelberg, 2013; pp 85-110.

[33] McCahill, J. S. J.; Welch, G. C.; Stephan, D. W., Angew. Chem., Int. Ed. 2007, $46(26), 4968-4971$.

[34] Mömming, C. M.; Kehr, G.; Wibbeling, B.; Fröhlich, R.; Erker, G., Dalton Trans. 2010, 39 (32), 7556-7564.

[35] Mömming, C. M.; Frömel, S.; Kehr, G.; Fröhlich, R.; Grimme, S.; Erker, G., J. Am. Chem. Soc. 2009, 131 (34), 12280-12289.

[36] Stephan, D. W., Org. Biomol. Chem. 2008, 6 (9), 1535-1539.

[37] Sajid, M.; Klose, A.; Birkmann, B.; Liang, L.; Schirmer, B.; Wiegand, T.; Eckert, H.; Lough, A. J.; Frohlich, R.; Daniliuc, C. G.; Grimme, S.; Stephan, D. W.; Kehr, G.; Erker, G., Chem. Sci. 2013, 4 (1), 213-219.

[38] Peuser, I.; Neu, R. C.; Zhao, X.; Ulrich, M.; Schirmer, B.; Tannert, J. A.; Kehr, G.; Fröhlich, R.; Grimme, S.; Erker, G.; Stephan, D. W., Chem. Eur. J. 2011, 17 (35), 9640-9650. 
[39] Welch, G. C.; Juan, R. R. S.; Masuda, J. D.; Stephan, D. W., Science 2006, 314 (5802), 1124-1126.

[40] Rokob, T. A.; Hamza, A.; Pápai, I., J. Am. Chem. Soc. 2009, 131 (30), 10701 10710.

[41] Chase, P. A.; Jurca, T.; Stephan, D. W., Chem. Commun. 2008, (14), 17011703.

[42] Wang, H.; Fröhlich, R.; Kehr, G.; Erker, G., Chem. Commun. 2008, (45), 5966-5968.

[43] Spies, P.; Schwendemann, S.; Lange, S.; Kehr, G.; Fröhlich, R.; Erker, G., Angew. Chem., Int. Ed. 2008, 47 (39), 7543-7546.

[44] Scott, D. J.; Fuchter, M. J.; Ashley, A. E., Angew. Chem., Int. Ed. 2014, 53 (38), 10218-10222.

[45] Scott, D. J.; Fuchter, M. J.; Ashley, A. E., J. Am. Chem. Soc. 2014, 136 (45), 15813-15816.

[46] Mahdi, T.; Stephan, D. W., J. Am. Chem. Soc. 2014, 136 (45), 15809-15812.

[47] Gyömöre, Á.; Bakos, M.; Földes, T.; Pápai, I.; Domján, A.; Soós, T., ACS Catalysis 2015, 5 (9), 5366-5372.

[48] Bertini, F.; Lyaskovskyy, V.; Timmer, B. J. J.; de Kanter, F. J. J.; Lutz, M.; Ehlers, A. W.; Slootweg, J. C.; Lammertsma, K., J. Am. Chem. Soc. 2012, 134 (1), 201-204.

[49] Stephan, D. W.; Erker, G., Chem. Sci. 2014, 5 (7), 2625-2641.

[50] Ménard, G.; Gilbert, T. M.; Hatnean, J. A.; Kraft, A.; Krossing, I.; Stephan, D. W., Organometallics 2013, 32 (15), 4416-4422.

[51] Zhao, X.; Stephan, D. W., Chem. Commun. 2011, 47 (6), 1833-1835.

[52] Theuergarten, E.; Bannenberg, T.; Walter, M. D.; Holschumacher, D.; Freytag, M.; Daniliuc, C. G.; Jones, P. G.; Tamm, M., Dalton Trans. 2014, 43 (4), 16511662 .

[53] Chapman, A. M.; Haddow, M. F.; Wass, D. F., J. Am. Chem. Soc. 2011, 133 
(45), 18463-18478.

[54] Lu, Z.; Wang, Y.; Liu, J.; Lin, Y.-j.; Li, Z. H.; Wang, H., Organometallics 2013, $32(22), 6753-6758$.

[55] Courtemanche, M.-A.; Légaré, M.-A.; Maron, L.; Fontaine, F.-G., J. Am. Chem. Soc. 2014, $136(30), 10708-10717$.

[56] Chen, E. Y.-X., Polymerization by Classical and Frustrated Lewis Pairs. In Frustrated Lewis Pairs II: Expanding the Scope, Erker, G.; Stephan, D. W., Eds. Springer Berlin Heidelberg: Berlin, Heidelberg, 2013; pp 239-260.

[57] Hong, M.; Chen, J.; Chen, E. Y. X., Chem. Rev. 2018, 118 (20), 10551-10616.

[58] Zhang, Y.; Miyake, G. M.; Chen, E. Y.-X., Angew. Chem., Int. Ed. 2010, 49 (52), 10158-10162.

[59] Wang, Q.; Zhao, W.; Zhang, S.; He, J.; Zhang, Y.; Chen, E. Y. X., ACS Catalysis 2018, 8 (4), 3571-3578.

[60] Li, X.; Chen, C.; Wu, J., Molecules 2018, 23 (1), 189.

[61] Carolyn, D., Smart Materials and Structures 1994, 3 (2), 118.

[62] Dry, C., Compos. Struct. 1996, 35 (3), 263-269.

[63] White, S. R.; Sottos, N. R.; Geubelle, P. H.; Moore, J. S.; Kessler, M. R.; Sriram, S. R.; Brown, E. N.; Viswanathan, S., Nature 2001, 409, 794.

[64] Toohey, K. S.; Sottos, N. R.; Lewis, J. A.; Moore, J. S.; White, S. R., Nature Mater. 2007, 6, 581.

[65] Hansen, C. J.; Wu, W.; Toohey, K. S.; Sottos, N. R.; White, S. R.; Lewis, J. A., Adv. Mater. 2009, 21 (41), 4143-4147.

[66] Brown, E. N.; White, S. R.; Sottos, N. R., Compos. Sci. Technol. 2005, 65 (15), 2474-2480.

[67] Vahedi, V.; Pasbakhsh, P.; Piao, C. S.; Seng, C. E., Journal of Materials Chemistry A 2015, 3 (31), 16005-16012.

[68] Blaiszik, B. J.; Kramer, S. L. B.; Olugebefola, S. C.; Moore, J. S.; Sottos, N. R.; White, S. R., Ann. Rev. Mater. Res. 2010, 40 (1), 179-211. 
[69] Kloxin, C. J.; Scott, T. F.; Adzima, B. J.; Bowman, C. N., Macromolecules 2010, $43(6), 2643-2653$.

[70] Chen, X.; Dam, M. A.; Ono, K.; Mal, A.; Shen, H.; Nutt, S. R.; Sheran, K.; Wudl, F., Science 2002, 295 (5560), 1698-1702.

[71] Chung, C.-M.; Roh, Y.-S.; Cho, S.-Y.; Kim, J.-G., Chem. Mater. 2004, 16 (21), 3982-3984.

[72] Canadell, J.; Goossens, H.; Klumperman, B., Macromolecules 2011, 44 (8), 2536-2541.

[73] Lei, Z. Q.; Xiang, H. P.; Yuan, Y. J.; Rong, M. Z.; Zhang, M. Q., Chem. Mater. 2014, 26 (6), 2038-2046.

[74] Rekondo, A.; Martin, R.; Ruiz de Luzuriaga, A.; Cabanero, G.; Grande, H. J.; Odriozola, I., Materials Horizons 2014, 1 (2), 237-240.

[75] Zheng, P.; McCarthy, T. J., J. Am. Chem. Soc. 2012, 134 (4), 2024-2027.

[76] Cash, J. J.; Kubo, T.; Bapat, A. P.; Sumerlin, B. S., Macromolecules 2015, 48 (7), 2098-2106.

[77] Chen, W.-P.; Hao, D.-Z.; Hao, W.-J.; Guo, X.-L.; Jiang, L., Acs Appl. Mater. Interfaces 2017.

[78] Röttger, M.; Domenech, T.; van der Weegen, R.; Breuillac, A.; Nicolaÿ, R.; Leibler, L., Science 2017, 356 (6333), 62-65.

[79] Roberts, M. C.; Hanson, M. C.; Massey, A. P.; Karren, E. A.; Kiser, P. F., Adv. Mater. 2007, 19 (18), 2503-2507.

[80] Ogden, W. A.; Guan, Z., J. Am. Chem. Soc. 2018.

[81] Bosman, A. W.; Sijbesma, R. P.; Meijer, E. W., Materials Today 2004, 7 (4), 34-39.

[82] Miwa, Y.; Kurachi, J.; Kohbara, Y.; Kutsumizu, S., Communications Chemistry 2018, $1(1), 5$.

[83] Sun, J.-Y.; Zhao, X.; Illeperuma, W. R. K.; Chaudhuri, O.; Oh, K. H.; Mooney, D. J.; Vlassak, J. J.; Suo, Z., Nature 2012, 489, 133. 
[84] Varley, R., Ionomers as Self Healing Polymers. In Self Healing Materials: An Alternative Approach to 20 Centuries of Materials Science, van der Zwaag, S., Ed. Springer Netherlands: Dordrecht, 2007; pp 95-114.

[85] Wei, Z.; He, J.; Liang, T.; Oh, H.; Athas, J.; Tong, Z.; Wang, C.; Nie, Z., Polym. Chem. 2013, 4 (17), 4601-4605.

[86] Kalista, S. J.; Ward, T. C., Journal of The Royal Society Interface 2007, 4 (13), 405-411.

[87] Sijbesma, R. P.; Beijer, F. H.; Brunsveld, L.; Folmer, B. J. B.; Hirschberg, J. H. K. K.; Lange, R. F. M.; Lowe, J. K. L.; Meijer, E. W., Science 1997, 278 (5343), 1601-1604.

[88] Burattini, S.; Greenland, B. W.; Merino, D. H.; Weng, W.; Seppala, J.; Colquhoun, H. M.; Hayes, W.; Mackay, M. E.; Hamley, I. W.; Rowan, S. J., J. Am. Chem. Soc. 2010, 132 (34), 12051-12058.

[89] Fox, J.; Wie, J. J.; Greenland, B. W.; Burattini, S.; Hayes, W.; Colquhoun, H. M.; Mackay, M. E.; Rowan, S. J., J. Am. Chem. Soc. 2012, 134 (11), 5362-5368.

[90] Yang, L.; Tan, X.; Wang, Z.; Zhang, X., Chem. Rev. 2015, 115 (15), 7196-7239.

[91] van Gemert, G. M. L.; Peeters, J. W.; Söntjens, S. H. M.; Janssen, H. M.; Bosman, A. W., Macromol. Chem. Phys. 2012, 213 (2), 234-242.

[92] Tadano, K.; Hirasawa, E.; Yamamoto, H.; Yano, S., Macromolecules 1989, 22 (1), 226-233.

[93] Kalista, S. J.; Ward, T. C.; Oyetunji, Z., Mechanics of Advanced Materials and Structures 2007, 14 (5), 391-397.

[94] Tachino, H.; Hara, H.; Hirasawa, E.; Kutsumizu, S.; Tadano, K.; Yano, S., Macromolecules 1993, 26 (4), 752-757.

[95] Varley, R. J.; Shen, S.; van der Zwaag, S., Polymer 2010, 51 (3), 679-686.

[96] Varley, R. J.; van der Zwaag, S., Polym. Int. 2010, 59 (8), 1031-1038.

[97] Atkins, P.; Paula, J. D., Atkins' Physical Chemistry. 9th ed.; W.H. Freeman and Company: United States, 2010; p 640. 
[98] Colquhoun, H. M.; Zhu, Z.; Cardin, C. J.; Gan, Y., Chem. Commun. 2004, (23), 2650-2652.

[99] Colquhoun, H. M.; Zhu, Z., Angew. Chem., Int. Ed. 2004, 43 (38), 5040-5045.

[100] Colquhoun, H. M.; Zhu, Z.; Cardin, C. J.; Gan, Y.; Drew, M. G. B., J. Am. Chem. Soc. 2007, 129 (51), 16163-16174.

[101] Greenland, B. W.; Burattini, S.; Hayes, W.; Colquhoun, H. M., Tetrahedron 2008, 64 (36), 8346-8354.

[102] Atkins, P.; Paula, J. D., Atkins' Physical Chemistry. 9th ed.; W.H. Freeman and Company: United States, 2010; p 631-636.

[103] Urban, M. W.; Davydovich, D.; Yang, Y.; Demir, T.; Zhang, Y.; Casabianca, L., Science 2018, 362 (6411), 220-225.

[104] Fontani, M.; Peters, F.; Scherer, W.; Wachter, W.; Wagner, M.; Zanello, P., Eur. J. Inorg. Chem. 1998, 1998 (10), 1453-1465.

[105] Grosche, M.; Herdtweck, E.; Peters, F.; Wagner, M., Organometallics 1999, 18 (22), 4669-4672.

[106] Christinat, N.; Croisier, E.; Scopelliti, R.; Cascella, M.; Röthlisberger, U.; Severin, K., Eur. J. Inorg. Chem. 2007, 2007 (33), 5177-5181.

[107] Sheepwash, E.; Krampl, V.; Scopelliti, R.; Sereda, O.; Neels, A.; Severin, K., Angew. Chem., Int. Ed. 2011, 50 (13), 3034-3037.

[108] Dodge, L.; Chen, Y.; Brook, M. A., Chem. Eur. J. 2014, 20 (30), 9349-9356.

[109] Qin, Y.; Cui, C.; Jäkle, F., Macromolecules 2007, 40 (5), 1413-1420.

[110] Vidal, F.; Lin, H.; Morales, C.; Jäkle, F., Molecules 2018, 23 (2), 405.

[111] Trunk, M.; Teichert, J. F.; Thomas, A., J. Am. Chem. Soc. 2017, 139 (10), $3615-$ 3618. 


\section{Chapter Two. Polymeric Frustrated Lewis Pairs from Boron and Phosphorous Containing Monomers}

\subsection{Introduction}

\subsubsection{Organoboron Chemistry}

Boron is the first element from group 13. It has an electron configuration of $1 s^{2}, 2 s^{2}$, $2 \mathrm{p}^{1}$, with three electrons in its valence orbitals. ${ }^{1}$ In most cases, when forming covalent bonds with other atoms, boron adopts an $\mathrm{sp} 2$ hybridisation to give three $\mathrm{sp}^{2}$ orbitals, leaving one $2 p$ orbital untouched. ${ }^{1}$ The three valence electrons will each occupy one $\mathrm{sp}^{2}$ orbitals and pair with the one from the incoming bonding atom. The empty $2 \mathrm{p}$ orbital is therefore not involved in the bond formation and will be able to accept one extra pair of electrons from a Lewis base (Scheme 2.1 (A)). ${ }^{1}$ For this reason, trivalent boron always behaves like a Lewis acid. Compared to other elements in Group XIII, boron has the highest electronegativity, meaning that its valence electrons tend to form localised covalent bonds rather than delocalised metallic bond. ${ }^{2}$ Boron is therefore the only non-metallic element in Group XIII, and is sometimes referred as a metalloid. ${ }^{2}$ Due to electron repulsion, the free unbound organoboron compounds adopt a near trigonal planar conformation. When a Lewis base coordinates, however, the boron compound will change its conformation to tetrahedral (Scheme 2.1 (B)). 
(A)

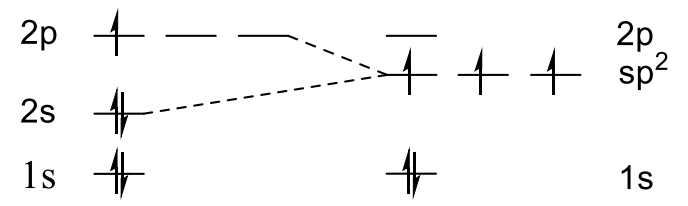

(B)<smiles>[R][R]([R])([R])[B][13B][B][R]([R])([R])[B]</smiles>

Scheme 2.1 Schematic representations of (A) hybridisation of boron in an organoboron compound and (B) conformational change of organoboron when coordinated by a Lewis base.

Boron exists in many different forms, both in nature or synthetic, e.g. as a single element, hydride, halide, ester, alkyl, aryl, etc. Scheme 2.2 below lists some common organoboron compounds, with their nomenclatures. The Lewis acidity of organoboron compounds depends to a large extent on the functional group attached, and generally is in order of boron trihalide $>$ triarylborane $>$ trialkylborane $>$ oxygen-derived boron.

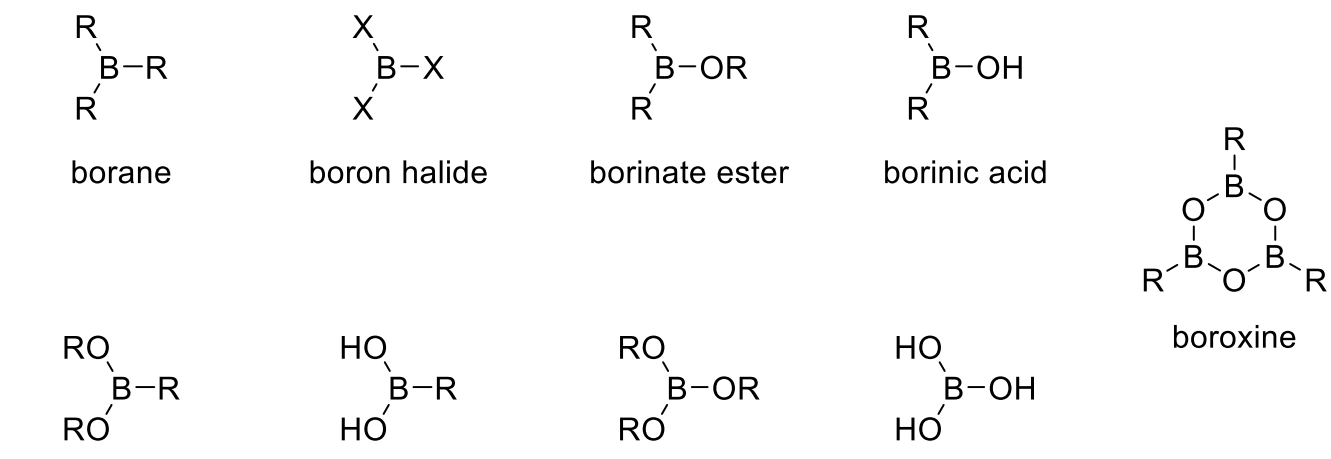

boronate ester boronic acid borate boric acid

Scheme 2.2 Common organoboron compounds. ${ }^{1}$

Because of their electron deficient nature, organoboron compounds in most cases are air-sensitive. For this reason, air-sensitive techniques are necessary for handling and synthesising them. The synthesis of organoboron compounds has been realised through many different strategies, e.g. Grignard reaction, organolithium reaction, hydroboration, metal-exchange reaction, etc. 
Organoboron has been incorporated into polymer chains, which has been achieved by either polymerisation of boron-containing monomers or post-polymerisation modifications. ${ }^{3}$ The former approach is usually thought to be preferred as it avoids the defects arising from incomplete modifications. The boron-containing monomers reported in the literature are often boronic acid or tetra-coordinated borane, which are less acidic. ${ }^{4}$ No example of high Lewis acidic triarylborane-containing monomers has been reported up to the date of this project, probably because of the difficult monomer synthesis and ensuing polymerisations that would be involved in the creation of such a monomer. In contrast, the post-polymerisation modification route has achieved more success, with related works primarily contributed by Jäkle et $a .^{3}$ The synthesis involves copolymerisation of styrene with 4-styryl-trimethylsilane, followed by boronsilicon exchange reactions to incorporate boron into the resultant polymer chains. Polymers containing various boron-moieties have been synthesised through this method, many of which are very reactive polymeric Lewis acids.

Since the discovery of organoboron compounds, their uses in CLPs and FLPs have been reported quantitatively in the literature. Apart from this, they have also been applied to many other fields. For example, in the area of organic synthetic chemistry, boronic acids have been found to be useful reagents in Suzuki-Miyaura coupling reactions for synthesising $\mathrm{C}-\mathrm{C}$ bonds. ${ }^{5-6}$ There are also examples of the use of Lewis acidic boron compound as initiators to polymerise methyl methacrylate. ${ }^{7}$ Polymeric organoboron, meanwhile, has been used as a macro-gelator for synthesising selfhealing materials, as stated in the previous chapter. Organoboron polymers have also showed good electronic properties, suggesting potential applications within electronic devices such as light-emitting diodes (LEDs), organic field-effect transistors (OFETs), or solar cells. ${ }^{8-11}$ 


\subsubsection{Organophosphorus Chemistry}

Phosphorus is a group 15 element, whose elemental form is usually as a white or red solid, depending on its precise structure. Phosphorus is highly reactive and can selfignite easily, so it does not exist in its elemental form in nature. ${ }^{12}$ Although phosphorus is in the same group with nitrogen, the former is always referred to as an inorganic element, while the latter is thought to be organic. One of the most substantial differences between nitrogen and phosphorus is their coordination number. Due to its larger size, phosphorus can form more than three bonds with other atoms, whereas nitrogen can only have a maximum coordination number of three when neutral. The coordination number for organophosphorus compounds has been discovered to vary from one to six, and the most stable oxidation states are +3 and +5 . In general, phosphorus prefers a higher valency. Hence a low valency phosphorous compound always tends to be oxidised. The stability depends largely on the functionalities attached to the phosphorus. For example, trialkylphosphines are very unstable in air and readily oxidised to trialkylphosphine oxide. The much less basic triarylphosphines, meanwhile, are much more tolerant to air at r.t., and can only be oxidised when heated.

Scheme 2.3 shown below lists some organophosphorus compounds, together with their nomenclatures.

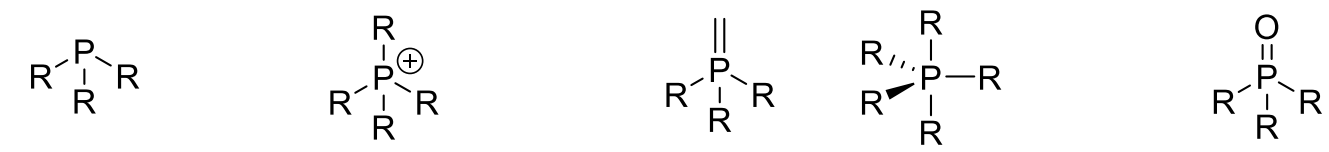

phosphine phosphonium ions phosphorane

phosphine oxide<smiles>[R]P([R])([R])=O</smiles>

phosphinate

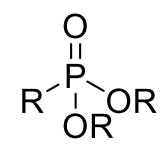

phosphonate<smiles>[R]P(=O)(O)O</smiles>

phosphonic acid<smiles>[R]OP([R])([R])=O</smiles>

phosphate<smiles>O=P(O)(O)O</smiles>

phosphoric acid

Scheme 2.3 Common organophosphorus compounds.

The most frequently studied organophosphorus compounds are phosphates, phosphines and their derivatives. Since the $\sigma$-donating ability of phosphorus in the 
phosphoryl group is quenched by oxygen, phosphines with low oxidation states are the compounds used most widely in Lewis pair chemistry. The Lewis basicity of phosphines is generally enhanced by the attachment of alkyl groups, except that in some cases too much steric hindrance is introduced to the phosphorus atoms.

Small molecule-based organophosphorus compounds have been widely used in both research and industry. Phosphines are incorporated in many synthetic processes. For example, they can form ylides and then react with ketones to form $\mathrm{C}=\mathrm{C}$ double bonds, which is commonly known as the Wittig reaction. ${ }^{13}$ They are also used as catalysts in Appel reactions to convert alcohols into alkyl chlorides. ${ }^{14}$ In Staudinger reduction reactions, meanwhile, they are used as the reagents to convert azides into amines. ${ }^{15}$ Thanks to their excellent $\sigma$-donating ability, phosphines are also frequently used as ligands in transition metal complexes. ${ }^{16}$ Phosphate derivatives are essential for the life process: they have been found in the backbone of DNA, as a critical part of a nucleotide. ${ }^{17}$ They have also been used as fertilisers in agriculture. In recent years, our group has also reported the first example of using phosphoric acids as catalysts for ring-opening polymerisations of cyclic ester monomers. ${ }^{18}$

The incorporation of phosphorus into polymer chains has also been studied quantitatively in the literature. Some very early work can be traced back to the 1970 s, in which phosphorus-containing polymers were prepared as macro-ligands to produce polymer-based metal complexes for catalysis. ${ }^{19-20}$ Polyphosphazenes have also been studied for their applications in high-performance elastomers and biomedicine, ${ }^{21-22}$ and there are reported examples of phosphate polymers for dental applications, and polyphosphoesters for drug delivery. ${ }^{23}$ Furthermore, the research in our group in recent years has discovered a series of organophosphorus polymers for optical applications. ${ }^{24-}$ 25 
This chapter discusses the synthesis of boron- and phosphorus-containing monomers and their polymerisations. The structure of the target monomers is shown below in Scheme 2.4. Two organophosphorus monomers were synthesised: 4-styryldimesitylphosphine (StPMes2) and 4-styryl-diphenylphosphine (StPPh2). The former is more sterically hindered due to the methyl groups attached at the ortho-positions of the mesitylene groups. The mesitylene groups can also improve the Lewis basicity of the whole phosphine molecule. StPMes2 is therefore expected to form a FLP with the target boron-containing monomer. The other phosphine monomer, $\mathbf{S t P P h}$, was synthesised for the purpose of a control experiment. Due to its reduced steric hindrance, StPPh2 should only form a CLP with the target boron-monomer. The boron-containing monomer $\mathbf{S t B P h} 2$ was synthesised in this work. Its non-fluorinated phenyl substituents provide an intermediate Lewis acidity to the boron centre, which potentially enables its dynamic binding with its Lewis base partner and activated bridging molecule. The detailed synthesis and characterisations are discussed in the following sections.<smiles>C=Cc1ccc(P(c2ccccc2)c2ccccc2)cc1</smiles><smiles>C=Cc1ccc(P(c2c(C)cc(C)cc2C)c2c(C)cc(C)cc2C)cc1</smiles><smiles>C=Cc1ccc(B(c2ccccc2)c2ccccc2)cc1</smiles>

Scheme 2.4 Structures of target phosphorus and boron-containing monomers. 


\subsection{Synthesis and Characterisation of the Phosphorus-/Boron-containing monomers}

\subsubsection{Lewis Basic Monomers}

\subsubsection{Synthesis of 4-styryl-diphenylphosphine (StPPh2)}

As stated above, the less sterically hindered phosphorus-containing monomer, 4styryl-diphenylphosphine (StPPh2), was synthesised since it can potentially bind to the target boron monomer for the control experiment. Its synthesis has been reported previously in the literature, which was performed using a Grignard reaction between diphenylphosphorous chloride ( $\mathbf{P h} 2 \mathbf{P C l})$ and 4-styrylmagnesium bromide (StMgBr). The former is a commercially available compound, while the latter was prepared from p-bromostyrene. The reaction is shown below in Scheme 2.5.<smiles>C=Cc1ccc(Br)cc1</smiles>

1. $0{ }^{\circ} \mathrm{C}, 30 \mathrm{~min}$ 2. r.t. $4 \mathrm{~h}$<smiles>C=Cc1ccc(C(C)(Br)Br)cc1</smiles>

StMgBr
$\mathrm{Ph}_{2} \mathrm{PC}$

1. $-78^{\circ} \mathrm{C}, 1 \mathrm{~h}$

2. $0^{\circ} \mathrm{C}, 1 \mathrm{~h}$

3. r.t. $3 \mathrm{~h}$

Yield: $70.3 \%$

Scheme 2.5 Synthesis of StPPh2.

The product was purified by column chromatography and characterised by NMR spectroscopy. The ${ }^{1} \mathrm{H}$ NMR spectrum clearly shows the existence of vinyl and aromatic protons with the expected relative integrals. The ${ }^{13} \mathrm{C}$ NMR spectrum shows three sets of phosphorus-coupled peaks, corresponding to ortho-, meta-, and ipso-aromatic carbons. Each set contains two doublets having the same coupling constants but differing in intensity. These correspond to the carbon atoms of the styryl and phenyl rings, respectively. The coupling constants of the three sets of resonances are 19.5, 
10.3 and $7.1 \mathrm{~Hz}$, which are assigned as ${ }^{1} J_{\mathrm{CP}},{ }^{2} J_{\mathrm{CP}}$, and ${ }^{3} J_{\mathrm{CP}}$, respectively. These phosphorus-carbon coupling values confirmed the attachment between the styryl group and the phosphorus atom. The ${ }^{31} \mathrm{P}$ NMR spectrum shows only one phosphorus environment, which gives a clear singlet peak at $-5.8 \mathrm{ppm}$. Both the relatively small ${ }^{1} J_{\mathrm{CP}}$ value $(<100 \mathrm{~Hz})$ in the ${ }^{13} \mathrm{C}$ NMR spectrum and a low chemical shift in the ${ }^{31} \mathrm{P}$ NMR spectrum confirms that the product obtained was tri-coordinated phosphorus with an oxidation state of + III.

\subsubsection{Synthesis of 4-Styryl-dimesitylphosphine (StPMes2)}

As stated before, 4-styryl-dimesitylphosphine (StPMes2) was synthesised due to its enhanced steric hindrance and higher estimated Lewis basicity compared to $\mathbf{S t P P h}$, which means it can potentially form an FLP with the target boron monomer. A similar synthetic strategy was applied for this monomer, shown in Scheme 2.6.

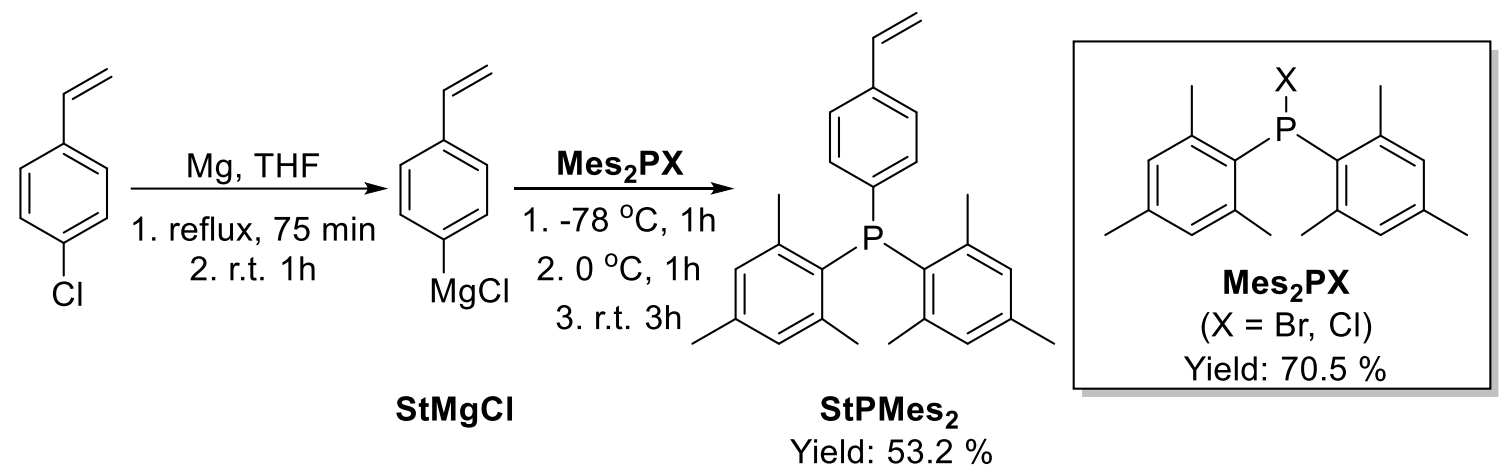

Scheme 2.6 Synthesis of StPMes2.

In this case, one of the starting materials, dimesitylphosphorous halide (Mes2 $\mathbf{P X})$, was not available commercially and need to be synthesised. This was successfully achieved by the reaction between the Grignard reagent, prepared from 2-bromomesitylene, and phosphorous trichloride. Since there are two different halogens involved in the reaction, the resultant product was a mixture of bromide and chloride due to competing halogenexchange reactions. Neither the chloride nor bromide derivatives of $\mathbf{M e s}_{2} \mathbf{P X}$ were 
found to affect the reactivity of the subsequent reaction, and were not purified further. The monomer StPMes2 was synthesised by the reaction between 4-styrylmagnesium chloride $(\mathbf{S t M g C l})$ and $\mathbf{M e s} 2 \mathbf{P X}$, with the former being prepared from 4-chlorostyrene. The solubility profile of the mesitylene-derived monomer, StPMes2, allowed for facile recrystallization from alcohols. However, StPMes2 was found easily oxidised at the elevated temperatures required for recrystallization. Therefore, it was purified by dissolving in THF and then precipitating from methanol. Removal of the oxidised byproduct was achieved by passing the crude product through a neutral aluminium oxide plug. Unlike its phenyl substituted analogue, StPMes2 was found degraded inside the silica column, an observation attributed to its higher basicity. The product was characterised by NMR spectroscopy. The ortho- and para- methyl groups in the aromatic rings of Mes2 $\mathbf{P X}$ exchanged their relative positions after attachment of the styryl group. ${ }^{13} \mathrm{C}$ NMR spectroscopy also confirmed the coupling between phosphorus and styryl carbon atoms. In this case, the carbon atoms in the phenyl and mesitylene groups have very distinct $J_{\mathrm{CP}}$ coupling constants. This is due to the very different chemical environments of the two aromatic rings. StPMes2 has a peak with a much lower chemical shift in the ${ }^{31} \mathrm{P}$ NMR spectrum compared to StPPh2 (-5.82 ppm), which is $-22.8 \mathrm{ppm}$.

\subsubsection{Determination of Lewis Basicity}

As stated in the previous chapter, the Lewis basicity of phosphines is inversely proportional to the ${ }^{1} J_{\mathrm{PSe}}$ value of their selenide derivatives. The relative basicity of the two phosphine monomers StPMes2 and StPPh2 were investigated by this method. Two phosphine selenides were synthesised by heating the two monomers with selenium pellets in chloroform under nitrogen overnight (Scheme 2.7). Any oxygen dissolved in solvent was removed by freeze-pump-thaw before the reaction. 


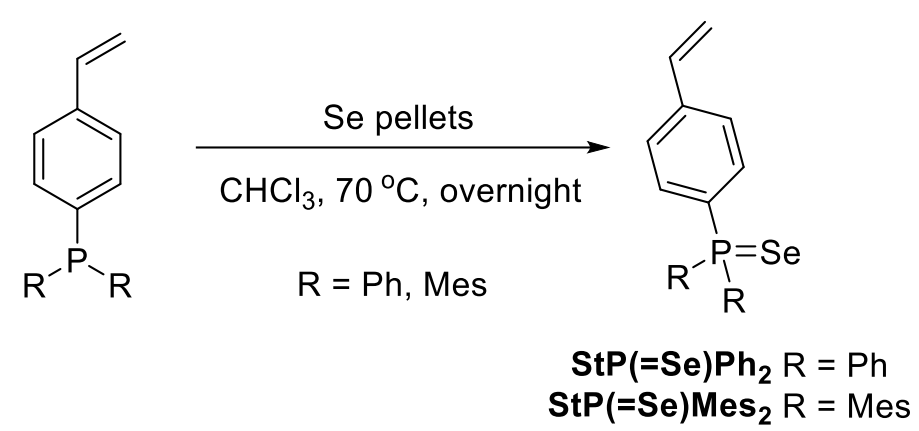

Scheme 2.7 Synthesis of $\operatorname{StP}(=\operatorname{Se}) \mathbf{P h} 2$ and $\operatorname{StP}(=$ Se)Mes2.

The obtained products were characterised by NMR spectroscopy. The ${ }^{31} \mathrm{P}$ NMR spectra show obvious deshielding of phosphorus peaks for both products. Changes in the chemical shifts were observed from $\delta-5.8$ to $\delta 34.7$ for $\mathbf{S t P}(=\mathbf{S e}) \mathbf{P h} 2$, and from $\delta-22.5$ to $\delta 19.4$ for $\mathbf{S t P}(=\mathbf{S e}) \mathbf{M e s}$ (Figure 2.1), confirming the bonding between selenium and phosphorus. The ${ }^{1} J_{\mathrm{PSe}}$ was measured to be $729.3 \mathrm{~Hz}$ for $\mathbf{S t P}(=\mathbf{S e}) \mathbf{P h} 2$ and 695.4 $\mathrm{Hz}$ for $\mathbf{S t P}(=\mathbf{S e}) \mathbf{M e s} 2$. According to the coupling constants, it was clear that the mesitylene substituted phosphine monomer is more Lewis basic than the phenyl substituted analogue, which is as expected. By comparing with the ${ }^{1} J_{\mathrm{PSe}}$ values of some phosphorous selenide compounds reported in the literature, ${ }^{26-27}$ the Lewis basicity of the two synthesised monomers can be summarised as in the order of $\mathrm{PCy}_{3}(672.9 \mathrm{~Hz})$ $>\mathrm{P}^{t} \mathrm{Bu}_{3}(692 \mathrm{~Hz})>\operatorname{StPMes} 2(695.4 \mathrm{~Hz})>\operatorname{PPhCy}_{2}(701.2 \mathrm{~Hz})>\operatorname{PPh}^{t} \mathrm{Bu}_{2}(708$ $\mathrm{Hz})>\mathrm{PPh}_{3}(728.9 \mathrm{~Hz}) \approx \mathbf{S t P P h}_{2}(729.3 \mathrm{~Hz})$. The phenyl substituted monomer StPPh2 shows a similar $\sigma$-donating ability to its analogue triphenylphosphine. For the mesitylene substituted monomer StPMes2, meanwhile, its Lewis basicity is similar to tris-tert-butylphoshpine $\left(\mathrm{P}^{t} \mathrm{Bu}_{3}\right)$, which is frequently used in various FLP systems. This indicated that the synthesised monomer StPMes2 should be able to act as an effective Lewis base partner in an FLP. It is also worth noting that the introduction of alkyl groups will dramatically increase the Lewis basicity of phosphine, and this might offer a way of designing new phosphorus-containing monomers in the future. 


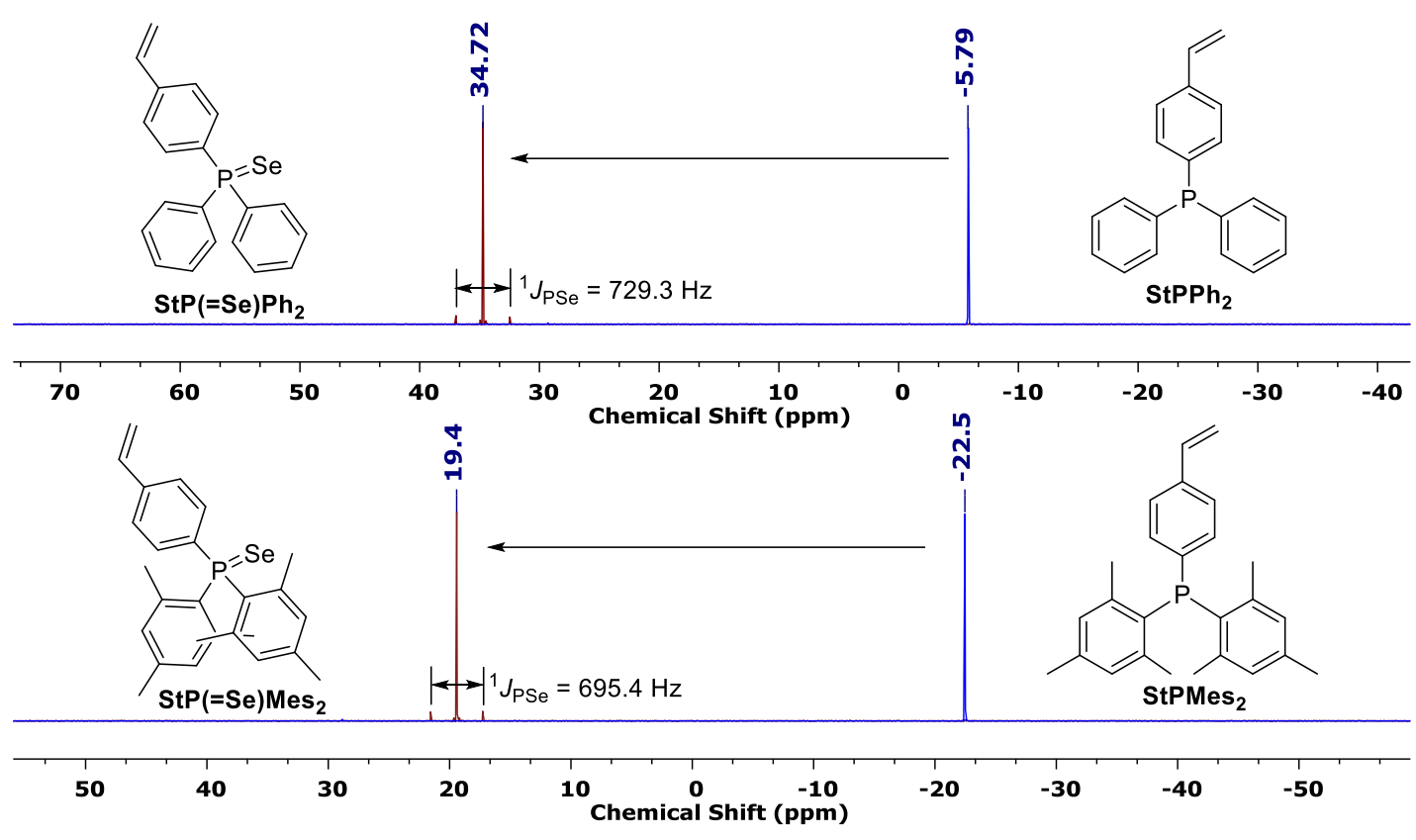

Figure 2.1 Lewis basicity determination using ${ }^{31} \mathrm{P}$ NMR spectroscopy.

\subsubsection{Lewis Acidic Monomers}

\subsubsection{Synthesis of 4-styryl-diphenylborane $\left(\mathrm{StBPh}_{2}\right)$}

Synthesis of an air-sensitive boron monomer is more challenging due to the sensitive vinyl group introduced and the difficult purification caused by the electron deficient boron moiety. Several factors were considered for this synthesis, including the competition between boron and any solvents/reagent and side reactions occurring at the reactive vinyl groups. The synthetic routes selected also should not be accompanied with too many side reactions, which increase the difficulty of product purification. Generally, organoboron compounds are synthesised by either nucleophilic substitution reactions or exchange reactions. In the case of the nucleophilic substitution route, the boron-containing precursors used are normally trialkyl borates or boron trihalides. Organolithium or Grignard reagents are frequently selected as the nucleophiles. Due to the existence of vinylphenyl groups in the target boron-containing monomer which are polymerisable, organolithium reagents were 
avoided since they have been reported to initiate polymerisations. The much milder Grignard reagents therefore were selected as nucleophiles. These, however, need the coordination of ether solvents to stabilise them, but they can bind to the electrondeficient boron monomer product as well. Triphenylborane, which is an analogue of the desired boron monomer, is known to be compatible with non-polar diethyl ether, which is less donating. Nonetheless, triphenylborane can still bind to the more polar tetrahydrofuran (THF). Using THF in the last step of boron monomer synthesis should therefore be avoided.

For the above reasons, from the beginning of attempts to synthesise 4-styryldiphenylborane $\mathbf{S t B P h}$, efforts were focused on exchange reactions since donating solvents or reagents can be avoided in these transformations. In 2002, Jäkle et al. synthesised a series of highly Lewis acidic boron-containing polymers via postpolymerisation modifications. ${ }^{3}$ The synthesis was based on a series of exchange reactions on copolymers containing unique functionalities. Inspired by this, a similar synthetic route was applied to the synthesis of the desired boron-containing monomer before the polymerisation of this monomer. This proposed synthetic route is shown below in Scheme 2.8 .

The reaction proceeded in multiple steps, which are listed in the following section. Firstly, in order to attach the boron moiety to styrene, a silicated styrene, 4-styryltrimethylsilane StSiMe3, needed to be synthesised. This was achieved via a Grignard reaction between $\mathbf{S t M g C l}$ and chlorotrimethylsilane. Secondly, the silicate substituent on StSiMe3 was to be modified to form the boron dibromide group to give dibromoborylstyrene StBBr2 via a boron-silicon exchange reaction. Because the boron halide can react with ether, the halogen moieties must be further modified to methoxy groups before the Grignard reaction could be attempted. StBBr2 therefore needs to react with chlorotrimethoxylsilane to give dimethoxyborylstyrene $\mathbf{S t B}(\mathrm{OMe}) 2$. Finally, 
a Grignard reagent was prepared from bromobenzene in diethyl ether and subsequently reacted with $\mathbf{S t B}(\mathbf{O M e})_{2}$ to give the desired boron monomer $\mathbf{S t B P h}$.

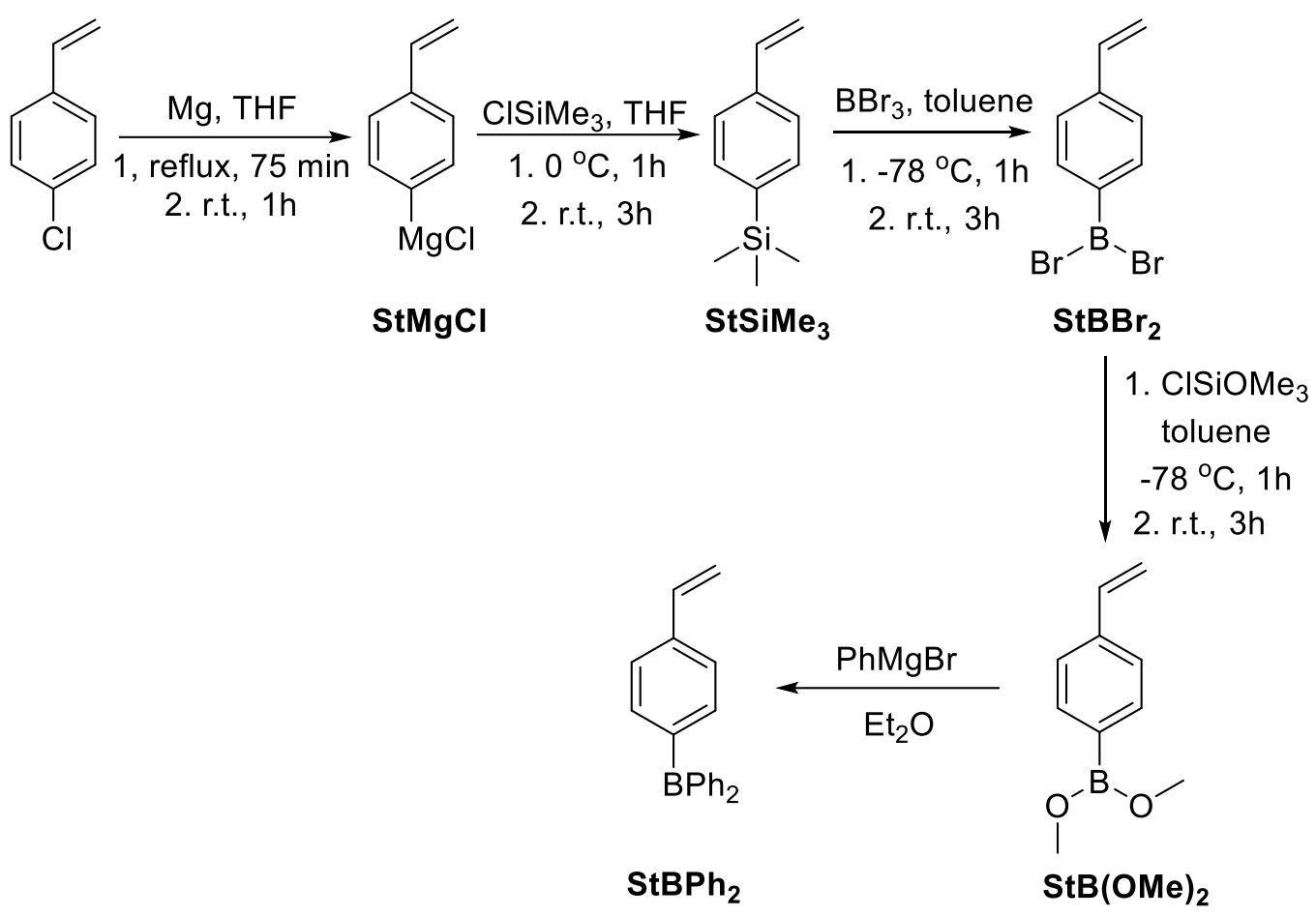

Scheme 2.8 Attempted synthesis of StBPh 2 via exchange reactions.

The synthesis of StSiMez has been widely reported in the literature and proceeded smoothly. The product can be purified by column chromatography, but for quantitative production as a monomer, it can also be purified by vacuum distillation. This product was used in the next step for the boron-silicon exchange reaction. The process was found both solvent and temperature dependent. Initially, similar reaction conditions to the literature were used, in which boron tribromide in DCM was added dropwise and mixed with StSiMe 3 at ambient temperature. This mixture was left to stir for $24 \mathrm{~h}$. The reaction did not work, however, and as a result, the vinyl group was found destroyed and lots of impurities generated. After vacuuming down the reaction vessel, the collected crude brown oil product did not show any peak in the ${ }^{11} \mathrm{~B}$ NMR spectrum, which also indicates the failure of boron attachment. The reaction temperature was reduced to $-78{ }^{\circ} \mathrm{C}$ and the reaction time was limited to $1 \mathrm{~h}$ in order to control the 
thermodynamics. Despite these changes, a similar result was obtained and no desired product was visible by spectroscopy (Figure 2.2 (B)). Afterwards, a milder Lewis acid, boron trichloride, was used instead. In this case, although the vinyl moieties were not harmed, the reaction became extremely slow, and multi-substitutions occurred before completion of reaction (Figure 2.2 (C)). Indeed, monitoring the ${ }^{1} \mathrm{H}$ NMR spectra, it was apparent that the conversion had only reached $40.5 \%$ after stirring at r.t. for $168 \mathrm{~h}$. Furthermore, after $24 \mathrm{~h}$ of reaction, di-substituted by-product started to appear, and even tri-substituted by-product could be seen after $144 \mathrm{~h}$ of reaction. From the relative integrals of the mono-/di-/tri- substituted products it was evident that the formation of di-/tri-substituted product is a more favourable process than the mono-substituted analogue. Following this attempt, the reaction was further modified, this time employing boron tribromide again but using toluene as the solvent. This combination of conditions led to the most selective reaction and generated desired intermediate product StBBr2 (Figure 2.2 (D)).

The reaction mixture was reduced in vacuo to remove any volatile components, and then the resultant product $\mathbf{S t B B r}$ was characterised using NMR spectroscopy. Using ${ }^{1} \mathrm{H}$ NMR spectroscopy, the characteristic peaks for the two aromatic protons at 7.42 ppm in the starting material, StSiMe3, were observed to shift significantly downfield to $8.20 \mathrm{ppm}$ after synthesis of StBBr2. This resulted from the attachment of a strong electron withdrawing boryl dibromide group, which was further confirmed by a new resonance at $56.3 \mathrm{ppm}$ in the ${ }^{11} \mathrm{~B}$ NMR spectrum. The ${ }^{29} \mathrm{Si}$ NMR spectrum showed the complete absence of any silicon-containing moieties since no peaks were observed. Following literature precedent, the product was used directly for the next step without further purification. 

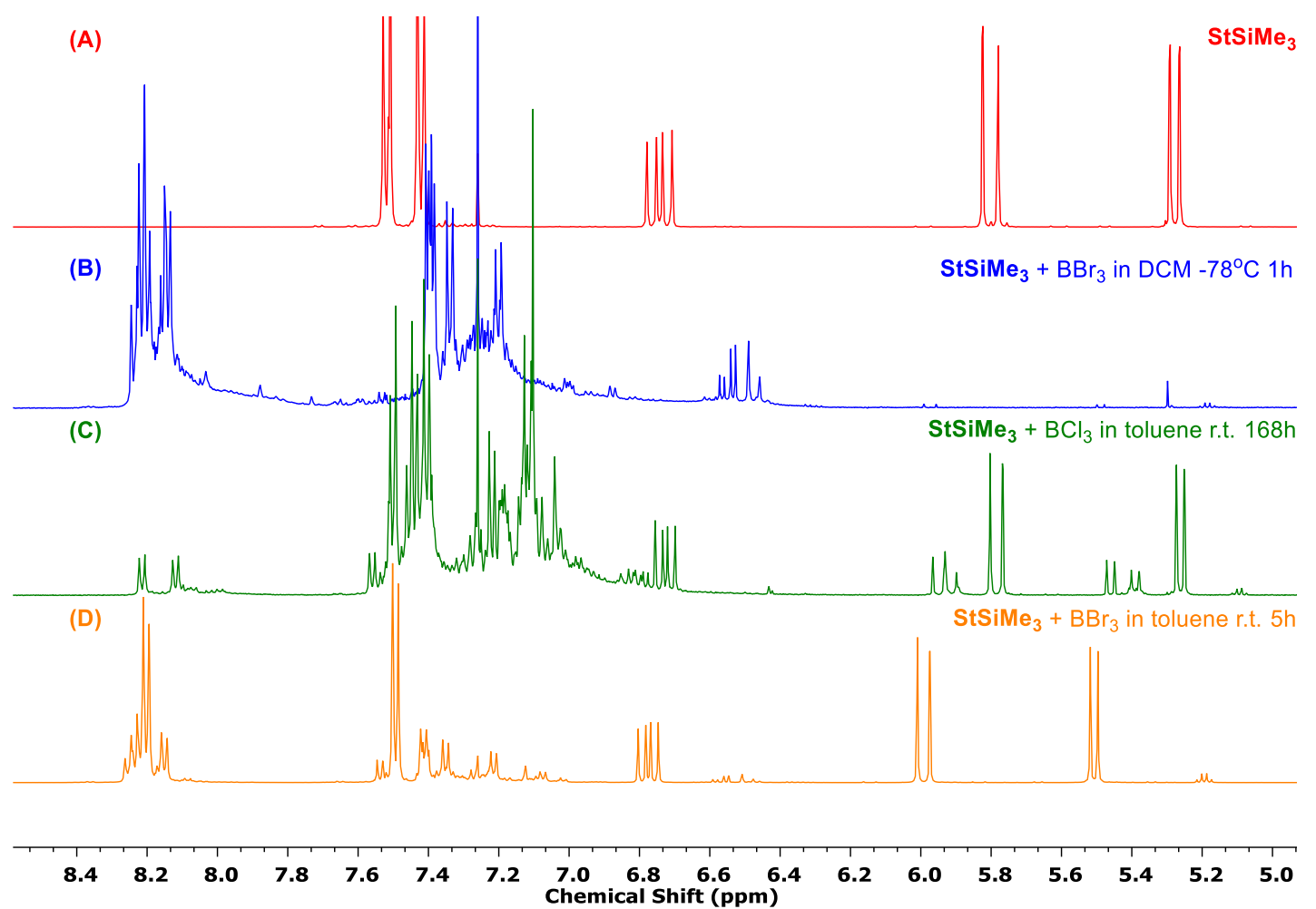

Figure 2.2 ${ }^{1} \mathrm{H}$ NMR spectra to show the boron-silicon exchange reactions between StSiMe 3 and boron trihalides. (A) StSiMe 3 before reaction; (B) $1 \mathrm{~h}$ after mixing of StSiMe 3 and $\mathbf{B B r}_{3}$ in DCM at $-78^{\circ} \mathrm{C}$; (C) $168 \mathrm{~h}$ after mixing of StSiMe 3 and $\mathbf{B C l}_{3}$ in toluene at r.t. and (D) $1 \mathrm{~h}$ after mixing of StSiMe3 and $\mathrm{BBr}_{3}$ in toluene at r.t.

The next step involved a boron-silicon exchange reaction between StBBr2 and chlorotrimethoxylsilane. This reaction was conducted in order to convert the boron halide group to boron methoxide in preparation for the following Grignard reaction. Unfortunately, no isolable product was obtained at this stage from this reaction. Although the ${ }^{11}$ B NMR spectrum showed consumption of the starting material, StBBr2, and generation of the target product $\mathbf{S t B}(\mathbf{O M e})_{2}$, the reaction was unselective and large amount of unidentified impurities were generated during the reaction. After removal of any volatiles under vacuum, the remaining crude product mixture was a viscous oil. Although a dramatic decrease in yield might be resulted, the distillation of this viscous oil was still performed in order to try to purify the product. However, only a light fog 
adsorbed onto the inner side of the distillation kits, and the majority of the oil turned to rigid solid afterwards. The difficulties in distilling the mixture was attributed to the high viscosity of the crude mixture. The reaction between this crude $\mathbf{S t B}(\mathbf{O M e})_{2}$ and the Grignard reagent $\mathbf{P h M g B r}$ was also attempted; again, however, the impurities generated in the previous two steps interrupted the reaction and no product could eventually be obtained. Due to the very distinct solubility parameters of polymeric material compared to small molecules, the purification of intermediates and final product were never problem when conducting post-polymerisation modifications. However, this was not the case in the reactions described above. This synthetic methodology was ceased, and another synthetic method was attempted.

There were two major problems that arose during the synthetic route stated above: the accumulation of impurities in each step that could not be easily removed, and the uncontrolled multi-substitutions on boron. To cope with these two problems, a boroncontaining intermediate product is required which already bears two phenyl rings and which can be purified before use. Such potential intermediate products include fluorodiphenylborane (FBPh2) and methoxyldiphenylborane (MeOBPh2), whose structures are shown below in Scheme 2.9.
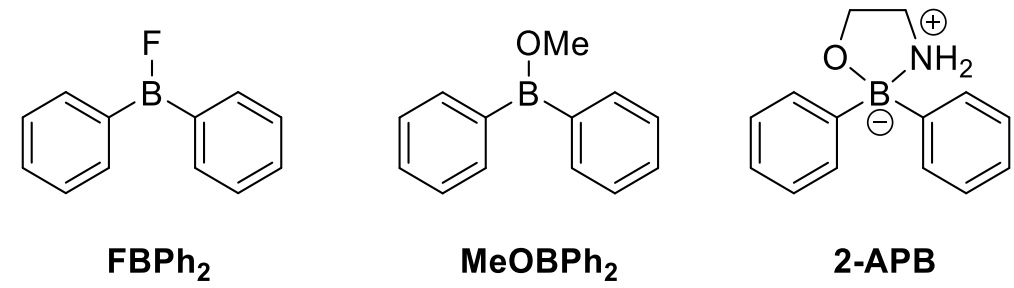

Scheme 2.9 Structures of FBPh2, MeOBPh2, and 2-APB.

Although these structures would be desirable candidates to use, 2-aminoethyldiphenyl borate (2-APB) was considered most appealing for the following reasons: Firstly, this compound requires less synthetic efforts compared to $\mathbf{F B P h} 2$ and MeOBPh2. FBPh2 
cannot be synthesised directly from boron trifluoride and organolithium because the reaction favours formation of triphenylborane hence cannot stop after substitution of the second phenyl group. Instead, a series of boron-silicon and halogen exchanges are required to synthesise it. Meanwhile, although $\mathbf{M e O B P h}$ is relatively easy to make, its major impurity, mono-substituted phenyldimethoxylborane, cannot be easily distilled off. Hence it is also not as suitable for use as 2-APB. Secondly, 2-APB is stable to air due to the self-protective binding between boron and its primary amine. The coordination makes it easy to handle and stored for long term use, which are both desirable for monomer synthesis. The self-stabilisation of 2-APB also dramatically changes the solubility of the compound, making it insoluble in most organic solvents except dimethyl sulfoxide (DMSO) and THF; hence it could be purified easily by simple precipitation methods. Typically, compounds bearing amine groups seem to be a problem in synthesising trivalent boron compounds, this moiety has been reported to be removable in the presence of a protic Brønsted acid.

The use of 2-APB as a boron precursor to the synthesis of donor-coordinated boron compounds has been reported by Tsang, Spadafora, Pomido and Patel in several patents. ${ }^{28-30}$ In their work, the excess amount of Grignard reagents were used to react with 2-APB to compete with the amine protons. An interesting procedure in their work-up was the addition of donating agents such as imidazole before the quenching of the Grignard reagents. The resultant product was protected by the donating agents hence will not be hydrolysed by the bulk water added. Also, the more donating imidazole prevented the coordination of solvent onto the boron. THF can therefore be used as a solvent for generating Grignard reagent. Their work inspired us to think about whether the boron monomer can be isolated as a coordinated derivative first and then recovered by removing the coordinating molecule. The synthesis of 4-styryldiphenylborane ammoniate $\left(\mathbf{S t B P h} \mathbf{P}_{2} \cdot \mathbf{N H}_{3}\right)$ was then attempted, shown below in Scheme 2.10. 


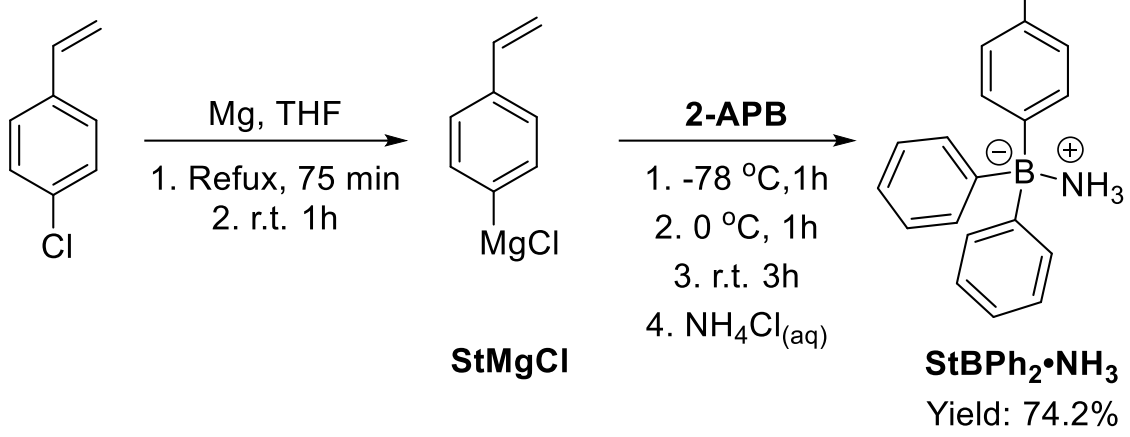

Scheme 2.10 Synthesis of $\mathrm{StBPh}_{2} \cdot \mathbf{N H}_{3}$.

Three equivalents of the Grignard reagent StMgCl was prepared and added into THF solution of 2-APB with careful control of temperature. It was found that the excess amount of Grignard reagent resulted in the displacement of the phenyl group to styryl. The electron impact mass (MS-EI) spectroscopy confirmed the existence of the byproduct di(4-vinylphenyl)phenylborane ammoniate. By low-temperature addition of Grignard reagent and gradual elevation of temperature, the reaction rate was found easily controlled to diminish any multi-substitution side-reactions. After completion of the reaction, the excess Grignard reagent was quenched using aqueous ammonia chloride $\left(\mathrm{NH}_{4} \mathrm{Cl}\right)$ solution directly, and no donating agent was added beforehand. Surprisingly, the triarylborane product was not hydrolysed during work-up but readily bound by the ammonia molecule generated from ionisation of $\mathrm{NH}_{4} \mathrm{Cl}$. The boron in the product $\mathbf{S t B P h} \cdot \mathbf{N H}_{3}$ is very bulky and tetra-coordinated, so the product is oxygen and moisture stable, allowing traditional work-up methods like extraction to be applied, making the purification easier. The coordination of ammonia also severely limits the solubility of $\mathbf{S t B P h} 2 \cdot \mathbf{N H}_{3}$ to only THF, DMSO and acetone, which enables the product to be readily purified by precipitation. The product was obtained as a white powder.

The product was characterised by NMR spectroscopy and MS-EI spectroscopy. The ${ }^{1} \mathrm{H}$ NMR spectrum clearly showed the disappearance of primary amine and ethylene protons from 2-APB and the presence of ammonia protons. In addition, only one set 
of vinyl peaks could be seen, indicated the successful prevention of multi-substitution. The ${ }^{11} \mathrm{~B}$ NMR spectrum showed only one boron environment, which gave a sharp peak at $-4.9 \mathrm{ppm}$. Both the narrow pattern of the peak and the low chemical shift indicated this boron is tetra-coordinated. The MS-EI spectrum did not show the peak corresponding to the ammonia coordinated product. Instead, a peak corresponding to ammonia free triarylborane was observed. The loss of ammonia could be a result of electron impact by the spectrometer.

When optimising the reaction, it was found that the amount of Grignard reagent did not have to be the standard three equivalents to $\mathbf{2 - A P B}$, rather it was found that the amount of Grignard reagent can be reduced by as much as to two equivalents compared to 2-APB without much loss in resultant yield $(74.2 \%)$. When less than two equivalents of StMgCl was used, however, the reaction did not give a pure product. In some cases only a very sticky oil or suspension that cannot be further purified was obtained. The proton NMR spectra showed a mixture of $\mathbf{S t B P h} 2$ and 2-APB, which indicated that the excess of one equivalent of Grignard reagent is essential in changing the chemical property of the 2 -aminoethyl groups and makes them suitable for the desired transformation.

The next step was the recovery of the desired monomer StBPh2 from its ammoniate coordinated derivative. A simple reaction was performed by mixing an excess amount of hydrogen chloride etherate solution with $\mathbf{S t B P h} \mathbf{P}_{2} \cdot \mathbf{N H}_{3}$, shown below in Scheme 2.11. 

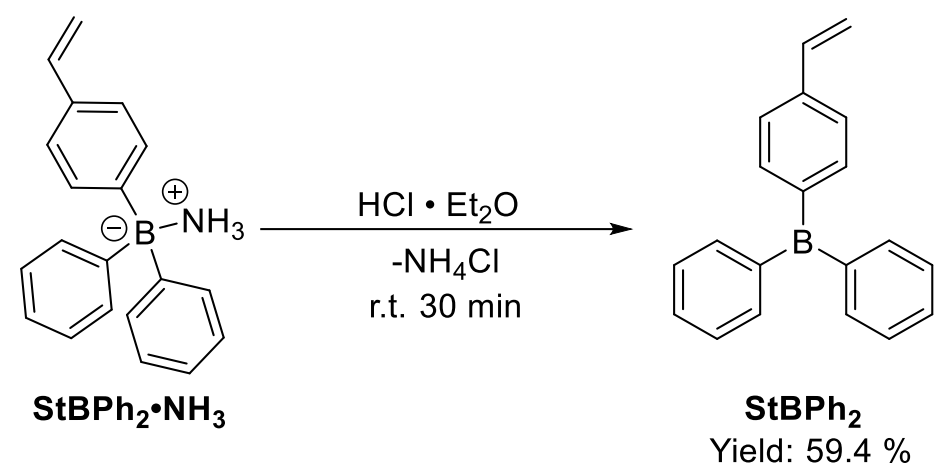

Scheme 2.11 Synthesis of StBPh2.

Upon mixing with hydrogen chloride, the relatively large clusters of the starting material $\mathbf{S t B P h} \mathbf{h}_{2} \cdot \mathbf{N H}_{3}$ quickly changed to a very fine powder, and heat was generated during the reaction. Apart from other acid-base equilibrium, this reaction was irreversible due to the poor solubility of $\mathrm{NH}_{4} \mathrm{Cl}$ in $\mathrm{Et}_{2} \mathrm{O}$. After a while, the solution changed colour to pale yellow due to some unavoidable hydrolysis by the small amount of water present in the hydrogen chloride solution. After the reaction, the solid was filtered off, and the product could then be readily purified by repeated recrystallisations from diethyl ether. The incorporation of the vinyl group dramatically increases the solubility of the boron compound. By subsequent investigations, it was found the more vinyl groups present, the more difficult recrystallisation was, often resulting in lower overal yields. This means the recrystallisation can also help to remove di-substituted by-product if small amount are present. The product $\mathbf{S t B P h} 2$ was characterised by NMR spectroscopy, the result of which is shown below in comparison with $\mathrm{StBPh}_{2} \cdot \mathbf{N H}_{3}$ in Figure 2.3. 


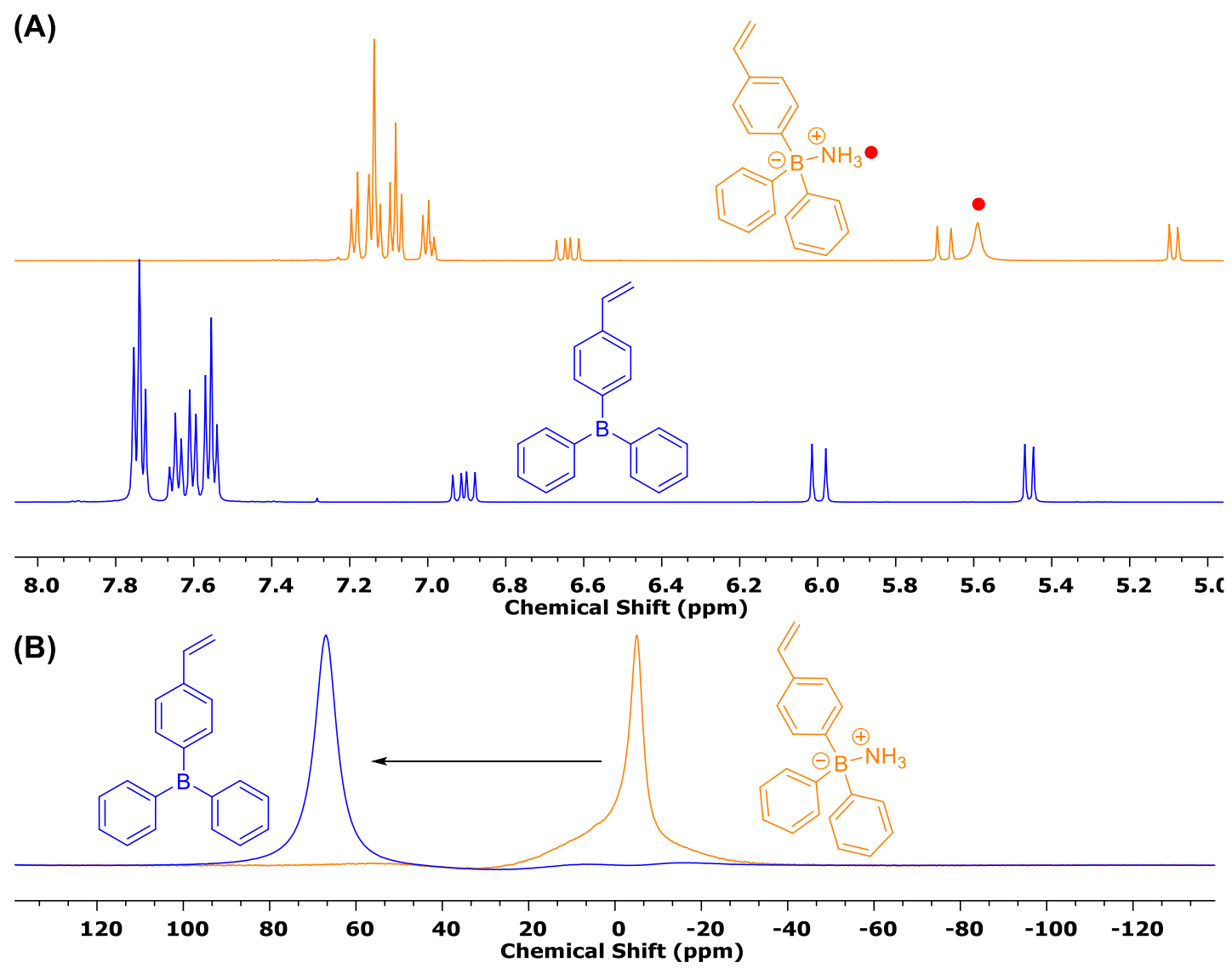

Figure 2.3 (A) ${ }^{1}$ H NMR spectra and (B) overlapped ${ }^{11}$ B NMR spectra of $\mathbf{S t B P h}$ and StBPh2 $\cdot \mathbf{N H}_{3}$.

From ${ }^{1} \mathrm{H}$ NMR spectra, it can be seen that the broad peak of the ammonia proton disappeared after the reaction, indicating successful removal of the coordinated base from the boron moiety. The chemical shifts associated with all moieties in the product displayed a downfield shift as the product protons were deshielded, due to the electron withdrawing effect by the unbound boron. The ${ }^{11} \mathrm{~B}$ NMR spectra also showed similar characteristics. After treatment with $\mathrm{HCl}$ etherate solution, the chemical shift of the boron peak was deshielded from -4.9 to $67.2 \mathrm{ppm}$, with the latter corresponding to a triarylborane. The peak also became much broader, indicating a free unbound boron centre. 
In summary, the target boron-containing monomer StBPh2 was successfully synthesised using a coordination-recovery strategy. Such a synthetic method not only dramatically reduces the difficulty of purification but also avoids the formation of multi-substituted by-products. Compared to multi-stepped exchange reactions, such a synthetic route is synthetically easy to perform, has minimal need of an air-sensitive technique, and also returns higher yields. Another advantage of this synthetic method is that the incorporation of a strong donating agent can protect the boron centre temporarily while being removed when required. This allows a strong donating solvent, such as THF, to be used during the reaction, which is normally avoided in organoboron synthesis. The two key intermediate products, $\mathbf{2 - A P B}$ and $\mathbf{S t B P h} \cdot \mathbf{N H} \mathbf{N H}_{3}$ are both airstable and easy to handle, which allow long term storage under air for at least several months. Their ease of purification ensures the successful synthesis of the monomer. The same synthetic strategy was also successfully applied for synthesising non-/di-/tristyryl substituted triarylborane compounds, shown below in Scheme 2.12.<smiles>c1ccc(B(c2ccccc2)c2ccccc2)cc1</smiles><smiles>C=Cc1ccc(B(c2ccccc2)c2ccccc2)cc1</smiles><smiles>C=Cc1ccc(B(c2ccccc2)c2ccc(C=C)cc2)cc1</smiles><smiles>C=Cc1ccc(B(c2ccc(C=C)cc2)c2ccc(C=C)cc2)cc1</smiles>

Scheme 2.12 Triarylborane synthesised using coordination-recovery strategy.

\subsubsection{Determination of Lewis Acidity}

The Gutmann-Beckett method was used to determine the Lewis acidity of the boroncontaining monomers synthesised in this project. Firstly, 1:1 equivalent amount of triethylphosphine oxide and StBPh2 were dissolved in deuterated DCM and characterised by NMR spectroscopy. The change in the chemical shift was observed in the ${ }^{31} \mathrm{P}$ NMR spectrum, shown below in Figure 2.4. 


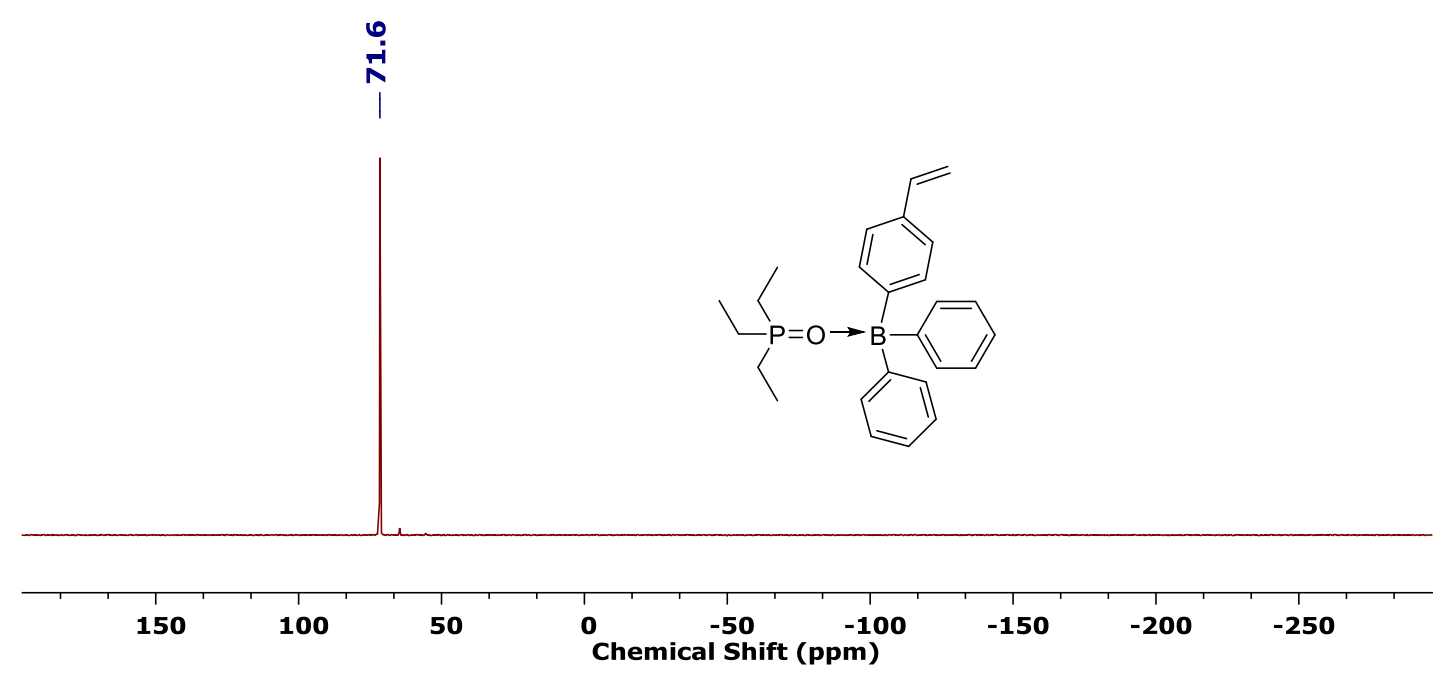

Figure 2.4 Determination of Lewis acidity of $\mathbf{S t B P h}$.

The acceptor number (AN) was calculated as 67.6, which was very similar to the literature value of its analogue, triphenylborane. This indicates that the synthesised boron monomer, unsurprisingly, has similar reactivity compared to triphenylborane. Although this synthesised boron monomer should be defined as a mild Lewis acid, our subsequent researches showed it successfully formed a binding-complex with the phosphine monomers by activating a small molecule diethyl azodicarboxylate (DEAD), discussed later in Chapter Three.

\subsection{Polymerisations}

\subsubsection{Polymerisation of Lewis Basic Monomers}

Both StPPh2 and StPMes2 have been copolymerised with styrene in this project, with the latter monomer being studied more extensively. The polymerisation method was initially determined to be atom transfer radical polymerisation (ATRP) since this mechanism introduces a halide atom at the chain end only after polymerisation, which does not affect Lewis acids. The catalyst used was copper (I) bromide with $N, N, N$ 'N", $N$ '-pentamethyldiethylenetriamine (PMDETA) as the ligand, and (1- 
bromoethyl)benzene was selected as the initiator. After several attempts with the monomer StPPh2, however, an insoluble gel was always formed at the end of polymerisation. Since all reagents were successful with the homo-polymerisation of styrene, it was concluded the phosphine monomer was not compatible with this polymerisation method. Exploring the literature, it was discovered that Poli et al. had also reported this issue. ${ }^{31}$ They proposed that in the presence of phosphine monomers, the alkyl halide groups at the polymer chain-ends can be either nucleophilically attacked to form quaternary phosphonium salts, or dehydrobrominated to generate alkenes. The former event can act as a linkage, while the latter was polymerisable, hence both resulted in hyperbranched polymers (Scheme 2.13). ${ }^{31}$
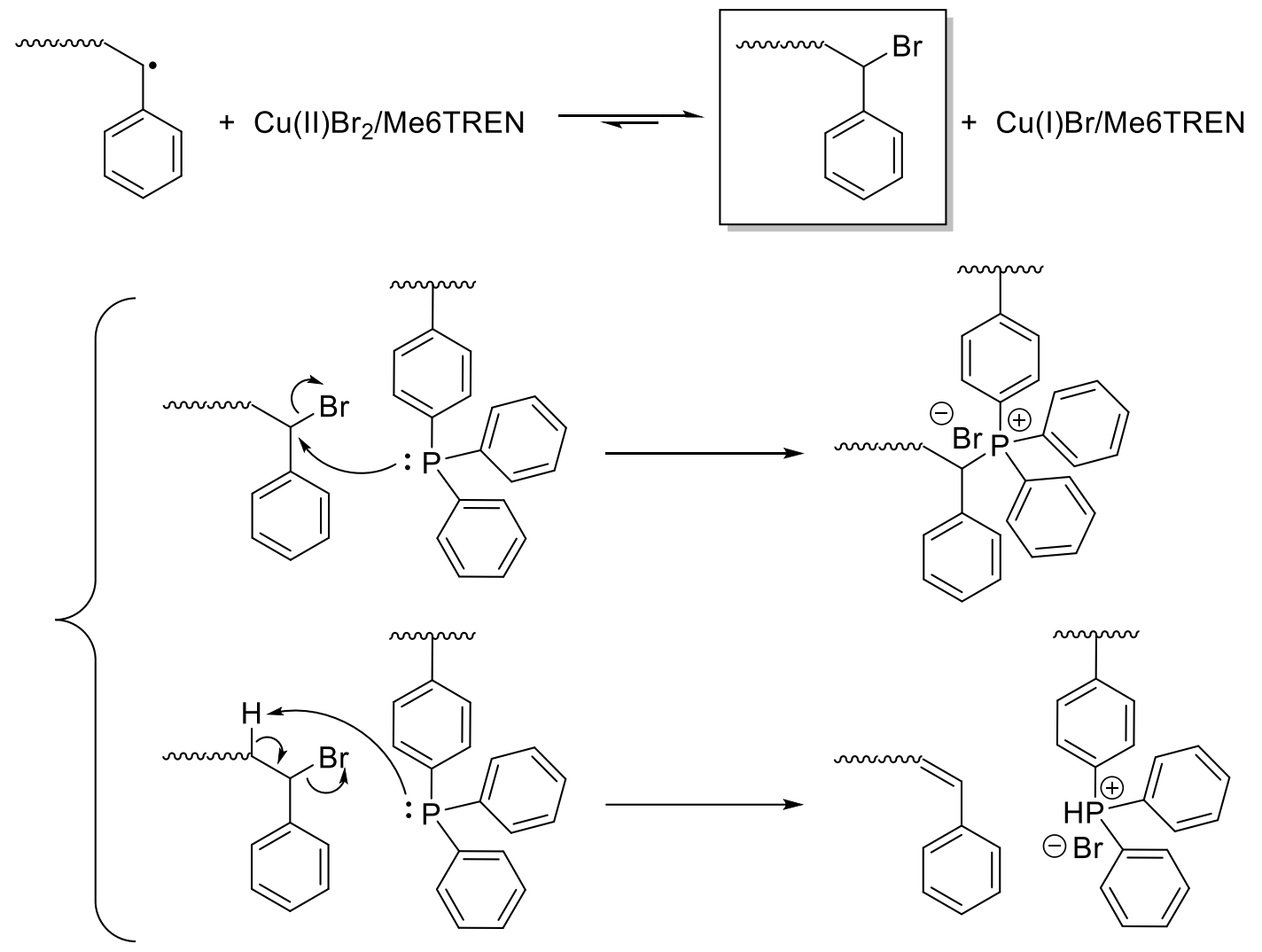

Scheme 2.13 Side reactions between phosphine monomer and polymer chain end. ${ }^{31}$

Due to the side reactions stated above, we decided to move to another controlled polymerisation method, reversible addition fragmentation chain-transfer polymerisation (RAFT), which is widely studied in polymer chemistry. The method 
involves using a chain-transfer agent as the catalyst to reach an equilibrium among propagating polymer chains. The chain transfer agent used in this project was cumyl dithiobenzoate (CDB), which is a widely used RAFT catalyst ideal for styryl-class monomers. For RAFT polymerisation to proceed, a radical initiator is required. Most of the commercially available initiators, such as azo-based compounds, contain functional groups that may bind with the boron moieties, however. Hence they were avoided. Although the compatibility of azobisisobutyronitrile (AIBN) to the synthesised boron monomer $\mathbf{S t B P h} 2$ was later confirmed by NMR spectroscopy, a system which involved minimum introduction of extra functionalities was always preferable. For this reason, the polymerisation was performed at a temperature of $110^{\circ} \mathrm{C}$, at which styrene can self-initiate. ${ }^{32}$ The RAFT copolymerisation of phosphine monomers with styrene is shown below in Scheme 2. 14 .

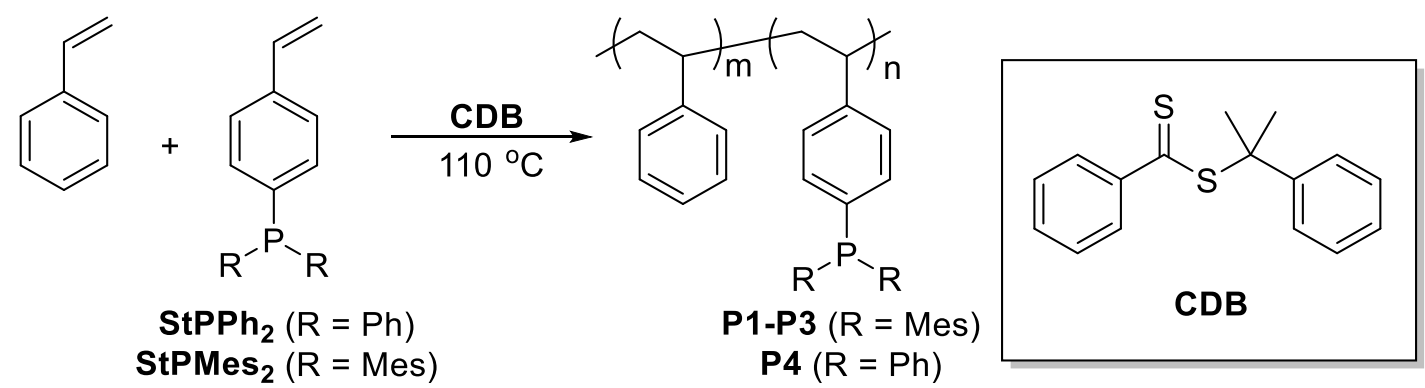

Scheme 2. 14 RAFT copolymerisation of phosphine monomers and styrene.

The polymerisation was performed by mixing phosphine monomer, styrene and CDB with calculated ratios, and the resultant polymerisation data is shown in Table 2.1. Since the phosphine monomer has very limited solubility, toluene was introduced in cases where more than $5 \%$ of phosphine monomers were used. The decrease in monomer concentration can result in a more extended self-inhibition period and slower polymerisation. A longer reaction time was therefore applied for toluene-containing polymerisations to make sure that all the resultant polymers had similar molecular weights. 
Table 2.1 RAFT copolymerisation of styrene and StPMes2, $\mathrm{T}=110^{\circ} \mathrm{C}$.

\begin{tabular}{|c|c|c|c|c|c|c|c|c|c|c|c|c|}
\hline \multirow{2}{*}{ Entry } & \multicolumn{3}{|c|}{ Feeding Ratio } & \multirow{2}{*}{$\begin{array}{l}\text { Tol } \\
v / v^{a}\end{array}$} & \multirow{2}{*}{$\begin{array}{c}\text { Time } \\
\text { h }\end{array}$} & \multicolumn{2}{|c|}{ Conversion } & \multicolumn{2}{|c|}{ In-Chain Ratio } & \multirow{2}{*}{$\begin{array}{l}M_{n, \text { theo }}{ }^{b} \\
\mathrm{~g} / \mathrm{mol}\end{array}$} & \multirow{2}{*}{$\begin{array}{l}M_{n, G P C}{ }^{c} \\
g / m o l\end{array}$} & \multirow{2}{*}{$\bigoplus$} \\
\hline & Styrene & StPMes $_{2}$ & CDB & & & Styrene & StPMes $_{2}$ & Styrene & StPMes $_{2}$ & & & \\
\hline P1 & 95 & 5 & 0.32 & - & 16 & 49.9 & 68.5 & 93.3 & 6.7 & 19661 & 20200 & 1.17 \\
\hline P2 & 90 & 10 & 0.28 & 0.4 & 20 & 41.3 & 57.2 & 86.7 & 13.3 & 21775 & 22900 & 1.08 \\
\hline P3 & 80 & 20 & 0.26 & 0.7 & 24 & 35.8 & 42.5 & 77.1 & 22.9 & 24296 & 27200 & 1.06 \\
\hline
\end{tabular}

${ }^{\mathrm{a}} \mathrm{v} / \mathrm{v}$ of toluene $=$ volume of toluene $/$ total volume of reaction mixture. ${ }^{\mathbf{b}} \mathrm{M}_{\mathrm{n}}$, theo $=([$ Styrene $] \times$ Conv.styrene + $\left[\right.$ StPMes$\left._{2}\right] \times$ Conv.StPMes2 $2 /[\mathrm{CDB}]+272.43 .{ }^{\mathbf{c}} \mathrm{M}_{\mathrm{n}, \mathrm{GPC}}$ was measured using $\mathrm{dn} / \mathrm{dc}=0.185$.

As shown above, copolymers P1-P3 were prepared using 5\%, 10\% and 20\% of sterically bulky monomer StPMes2. The conversion of phosphine monomers was obtained by comparing the relative integrals of the monomer and polymer peaks using the ${ }^{31} \mathrm{P}$ NMR spectra. The conversion of styrene was calculated by the relative integrals of the vinyl and polymer backbone proton peaks, together with the phosphine monomer conversions. The in-chain ratio of phosphine monomer was also derived from conversions for gelation experiments. From the polymerisation data, it is evident that the phosphine monomer was a bit more reactive relative to styrene, as expected. Although the resultant copolymers had slight gradients in monomer compositions, phosphine-containing repeating units were still distributed throughout the polymer chains. In all cases, the molecular weight determined by the gel permission chromatography (GPC) were found to be comparable to those theoretically calculated. The dispersity of copolymers obtained all had very low values $(<1.2)$. Both the good agreement in molecular weight and low dispersity values indicated a high level of control over polymerisations. The dispersity of the three polymers P1-P3 also had a trend displaying decreased values. This was in agreement with some other reported RAFT polymerisations, where the introduction of solvent can lead to slower but bettercontrolled polymerisations. Another copolymer, P4, was also synthesised using $\mathbf{S t P P h}$, with a slight change in reaction conditions. This was used for another gelation experiment, which is discussed later in Chapter Three Section 3.4. 


\subsubsection{Polymerisation of Lewis Acidic Monomers}

For the sake of consistency, RAFT copolymerisation was also applied to the boron monomer. Before polymerisation, a binding experiment was performed between the boron-containing monomer $\mathbf{S t B P h} 2$ and the chain transfer agent CDB. By monitoring the ${ }^{11} \mathrm{~B}$ NMR spectra, it was obvious that there is no binding between them at either room or polymerisation temperature. Unlike the copolymerisations discussed previously, conversions of StBPh2 and styrene cannot be determined by only one NMR experiment after the reaction. The conversion of the boron monomer cannot be obtained from the NMR spectrum directly from the ${ }^{11} \mathrm{~B}$ NMR spectroscopy due to the limitation of instrument. Also, StBPh2 and styrene gave overlapped aliphatic peaks in the ${ }^{1} \mathrm{H}$ NMR spectrum after polymerisation. Hence an internal standard was required for accurate determination of the conversion values. One of the common internal standards used for RAFT polymerisation is 1,3,5-trioxane. By NMR spectroscopy, however, it was found able to bind to the boron monomer. Hence a sterically bulky molecule 1,3,5-triisopropyl-2,4,6-trioxane (TIPT) was synthesised instead. The polymerisation was performed in a similar way to that of phosphine monomers, shown below in Scheme 2.15. The polymerisation data was shown below in Table 2.2.
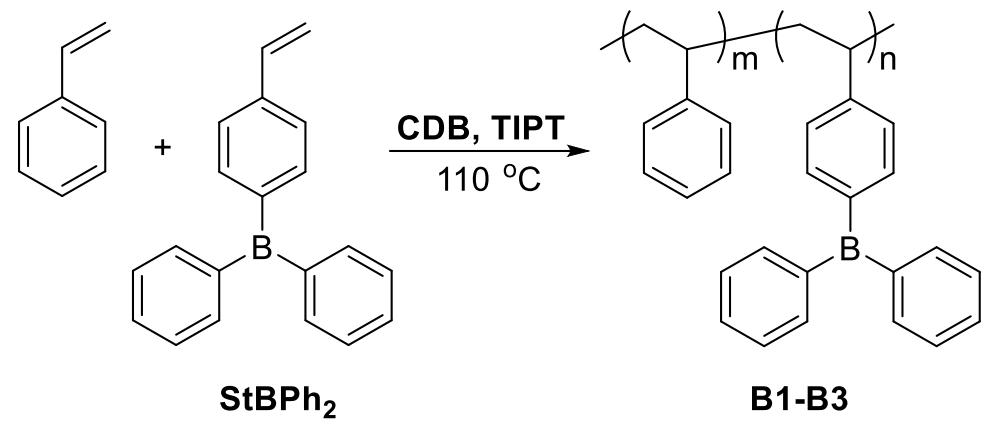

B1-B3

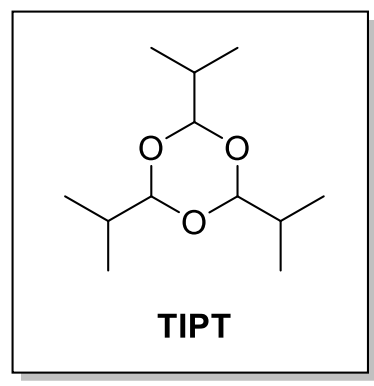

Scheme 2.15 RAFT copolymerisations of StBPh2 and styrene. 
Table 2.2 RAFT copolymerisations of styrene and $\mathbf{S t B P h}, \mathrm{T}=110^{\circ} \mathrm{C}$.

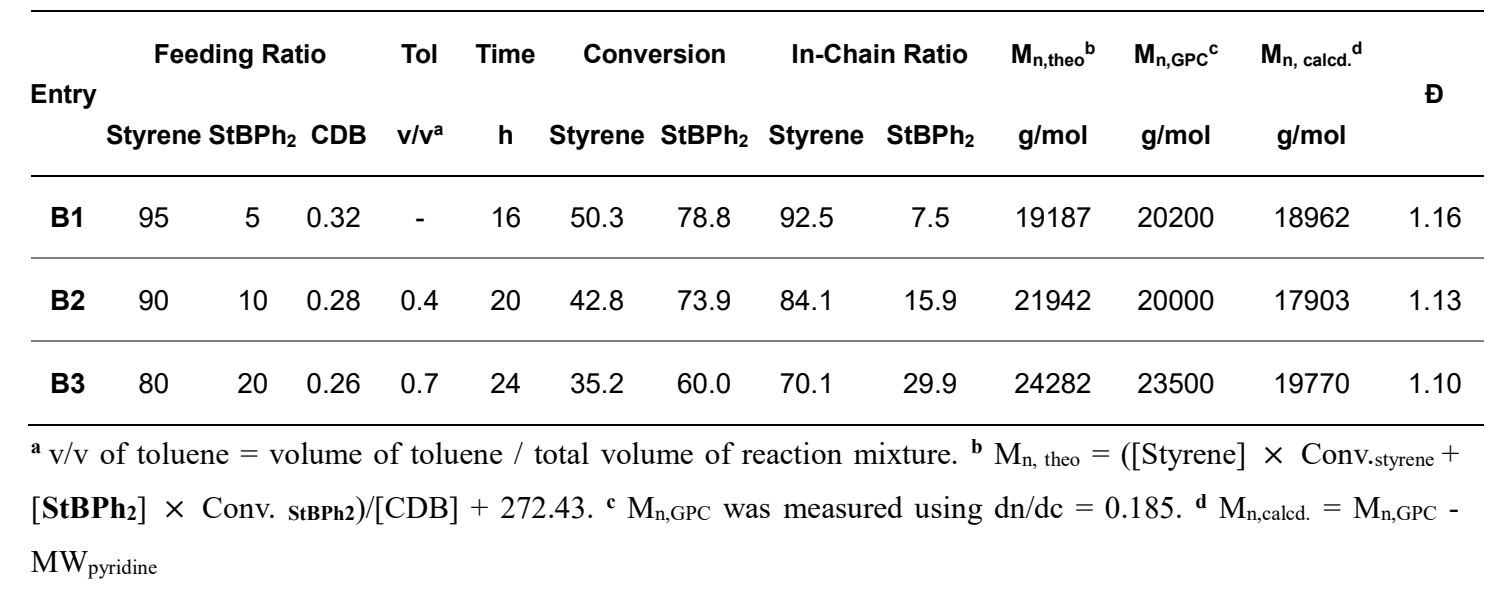

Similar to the copolymerisation of phosphine monomer, 5\%, 10\% and 20\% of $\mathbf{S t B P h} 2$ were used to produce copolymer B1-B3. By comparing the copolymerisations using the same amount of boron and phosphorus-containing monomers, it was found that the boron monomer was incorporated into the polymer chain at a slightly faster rate relative to phosphine monomers. The resultant in-chain ratios of boron and phosphorus are still comparable, meaning that this slight discrepancy should not dramatically affect the subsequent gelation experiments. Like phosphine-containing copolymers, B1-B3 should also be gradient copolymers but with a slightly larger variation in monomer compositions. The molecular weight values of resultant boron copolymers cannot be tested by GPC directly because of the potential binding between them and the guard column on the instrument. Hence the copolymers were first treated with pyridine to quench the Lewis acidity, then eluted by GPC. The actual molecular weight

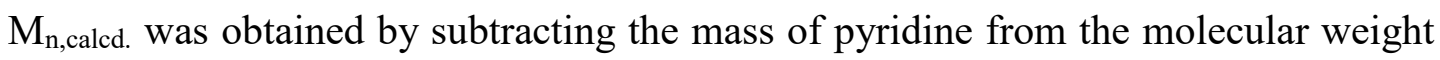
values obtained by GPC. Unlike phosphine copolymers, there was a discrepancy between theoretical and calculated molecular weights. Since this discrepancy increased with the amount of boron in the chain, it was postulated to result from the difference in $\mathrm{dn} / \mathrm{dc}$ values of boron-containing and styryl blocks. Nonetheless, the dispersity values were still very low, which indicated a good overall control of the polymerisations. The Lewis acidity of the obtained boron copolymer was also tested 
by the Gutmann-Beckett method, which found a decrease in $\mathrm{AN}\left(\mathrm{AN}=51.9\right.$ in $\mathrm{CDCl}_{2}$ and 58.5 in Toluene- $d_{8}$ ). Since the test was based on Lewis pair complexation, it was proposed that the lower AN was caused by the random coiled structure of the dissolved chain blocking coordination.

\subsection{Conclusion}

This chapter has reported the synthesis and characterisation of three monomers, two of which are novel. StPMes2 was sterically bulky, hence predicted to be able to form an FLP with a Lewis acid. This phosphine-containing monomer has a comparable $\sigma$ donating ability to tris-tert-butylphoshpine, which is a reagent frequently used in FLP chemistry. The less sterically bulky StPPh2 was also synthesised for the control experiment. A boron monomer $\mathbf{S t B P h} 2$ was synthesised via a novel coordinationrecovery strategy. The key intermediates were tetra-coordinated boron in order to reduce the difficulty of purification and handling. The desired tri-coordinated product was recovered by removal of the coordinated base using a Brønsted acid. Such a synthetic route proved successful and easy to perform, and is potentially applicable to other functionalised triarylboranes. The obtained boron monomer StBPh2 was analysed to determine their Lewis acidity, and found to be comparably acidic to its analogue, triphenylborane. Both the boron and phosphorus-containing monomers were copolymerised with the commercially available monomer, styrene, by RAFT copolymerisations. The polymerisations were well controlled to give copolymers with predicted molecular weight and narrow dispersity. The use of these copolymers for gelation experiments and the mechanical property characterisations of the resultant gels are discussed in the following chapter. 


\subsection{References}

[1] DeFrancesco, H.; Dudley, J.; Coca, A., Boron Chemistry: An Overview. In Boron Reagents in Synthesis, American Chemical Society: 2016; Vol. 1236, pp $1-25$.

[2] Norman, N. C., Periodicity and the s-and p-Block Elements. Oxford University Press: United States, 1997 Reprined 2011; p 34.

[3] Qin, Y.; Cheng, G.; Sundararaman, A.; Jäkle, F., J. Am. Chem. Soc. 2002, 124 (43), 12672-12673.

[4] Cheng, F.; Jäkle, F., Polym. Chem. 2011, 2 (10), 2122-2132.

[5] Miyaura, N.; Suzuki, A., Journal of the Chemical Society, Chemical Communications 1979, (19), 866-867.

[6] Miyaura, N.; Suzuki, A., Chem. Rev. 1995, 95 (7), 2457-2483.

[7] Erdyakov, S. Y.; Mel'nik, O. A.; Gurskii, M. E.; Ignatenko, A. V.; Vygodskii, Y. S., Russ. Chem. B. 2004, 53 (10), 2215-2220.

[8] Meng, B.; Ren, Y.; Liu, J.; Jäkle, F.; Wang, L., Angew. Chem., Int. Ed., n/a-n/a.

[9] Yin, X.; Guo, F.; Lalancette, R. A.; Jäkle, F., Macromolecules 2016, 49 (2), 537-546.

[10] Yin, X.; Chen, J.; Lalancette, R. A.; Marder, T. B.; Jäkle, F., Angew. Chem., Int. Ed. 2014, 53 (37), 9761-9765.

[11] Alahmadi, A. F.; Lalancette, R. A.; Jäkle, F., Macromol. Rapid. Comm. 2018, $39(22), 1800456$.

[12] Housecroft, C. E.; Sharpe, A. G., Inorganic Chemistry. 3rd ed.; Pearson Education Limited: United Kingdom, 2008; p 441-442.

[13] Clayden, J.; Greeves, N.; Warren, S.; Wothers, P., Organic Chemistry. Oxford University Press Inc.: United States, 2001; p 814-817.

[14] Appel, R., Angew. Chem. Int. Ed. Engl. 1975, 14 (12), 801-811.

[15] Staudinger, H.; Meyer, J., Helv. Chim. Acta 1919, 2 (1), 635-646. 
[16] Winter, M. J., d-Blcok Chemistry. Oxford University Press Inc.: United States, 1994 Reprined 2011; p 66-68.

[17] Albers, B.; Bray, D.; Hopkin, K.; Johnson, A.; Lewis, J.; Raff, M.; Roberts, K.; Walter, P., Essential Cell Biology. 2nd ed; Garland Science, Taylor \& Francis Group: United States and United Kingdom, 2004; p 169-176.

[18] Macdonald, E. K.; Shaver, M. P., Eur. Polym. J. 2017, 95, 702-710.

[19] Collman, J. P.; Hegedus, L. S.; Cooke, M. P.; Norton, J. R.; Dolcetti, G.; Marquardt, D. N., J. Am. Chem. Soc. 1972, 94 (5), 1789-1790.

[20] Grubbs, R. H.; Su, S.-C. H., J. Organomet. Chem. 1976, 122 (2), 151-157.

[21] Manners, I., Annual Reports Section "A" (Inorganic Chemistry) 1991, 88 (0), $77-92$

[22] Andrianov, A. K., Polyphosphazenes for Biomedical Applications. John Wiley \& Sons, Inc.: 2008.

[23] Monge, S.; Canniccioni, B.; Graillot, A.; Robin, J.-J., Biomacromolecules 2011, $12(6), 1973-1982$.

[24] Macdonald, E. K.; Shaver, M. P., Polym. Int. 2015, 64 (1), 6-14.

[25] Macdonald, E. K.; Lacey, J. C.; Ogura, I.; Shaver, M. P., Eur. Polym. J. 2017, $87,14-23$.

[26] Muller, A.; Otto, S.; Roodt, A., Dalton Trans. 2008, (5), 650-657.

[27] Beckmann, U.; Süslüyan, D.; Kunz, P. C., Phosphorus, Sulfur, and Silicon and the Related Elements 2011, 186 (10), 2061-2070.

[28] Tsang, T. H.; Spadafora, V. J.; Pomidor, P. Fungicidal Imidazole Diphenylaliphaticboranes And Derivatives Thereof. US Patent 4983589, Jan. 8, 1991.

[29] Patel, B. P. Diaryl(pyridinio and isoquinolinio) Boron Fungicidal Agents. US Patent 5354740, Oct. 11, 1994.

[30] Patel, B. P. Diaryl(pyridinio and isoquinolinio) Boron Insecticidal And Acaricidal Agents. US Patent 5354741, Oct. 11, 1994. 
[31] Cardozo, A. F.; Manoury, E.; Julcour, C.; Blanco, J.-F.; Delmas, H.; Gayet, F.; Poli, R., ChemCatChem 2013, 5 (5), 1161-1169.

[32] Mayo, F. R., J. Am. Chem. Soc. 1968, 90 (5), 1289-1295. 


\section{Chapter Three. Polymeric Frustrated Lewis Pairs as Network Macro-gelators}

\subsection{Binding experiments using monomers}

\subsubsection{Formation of Lewis Pair Adduct between $\mathrm{StPPh}_{2}$ and $\mathrm{StBPh}_{2}$ as Control Experiment}

Although bulky groups surround the activity centres of both monomers StPPh2 and StBPh2, some published work has indicated that they should be able to form a conventional Lewis pair (CLP) between each other. Our investigations confirmed this through a simple binding experiment, shown below in Scheme 3.1.

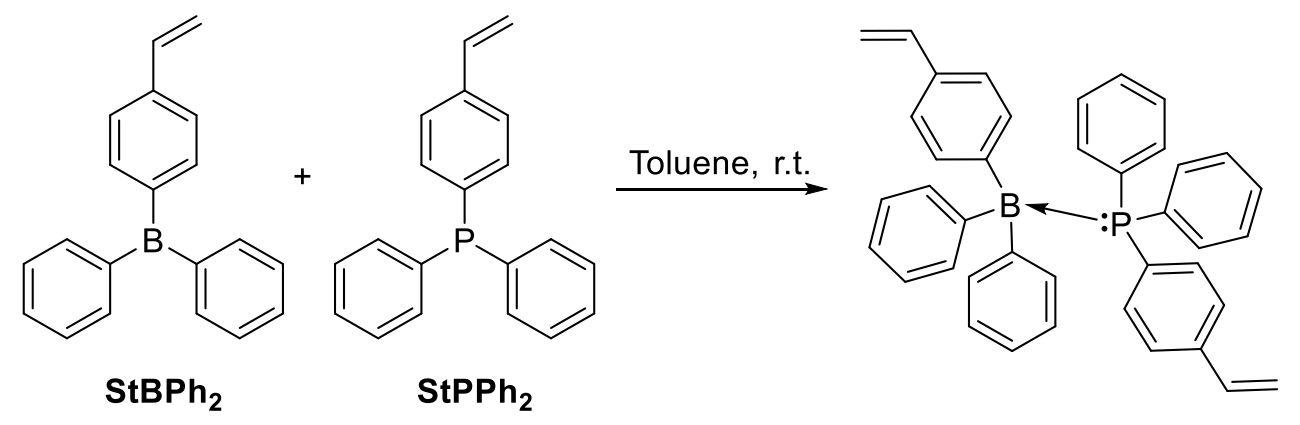

Scheme 3.1 Binding between StBPh2 and StPPh2.

Although the binding between these two monomers is predictable, the concentrationdependent nature of the observed binding was a striking observation. No binding was observed at low concentrations, but when the concentration was increased, an insoluble complex product was formed rapidly and precipitated out of the solvent. The obtained product was a white solid and air-stable, which indicated the protection of the boron group by phosphorus. This binding was not strong, however. The complex 
remained insoluble in common organic solvents, but disappeared when THF or DMSO was added. To monitor this process, the complex product was dissolved in deuterated DMSO and tested using ${ }^{11} \mathrm{~B}$ and ${ }^{31} \mathrm{P}$ NMR spectroscopy. Although the ${ }^{11} \mathrm{~B}$ NMR spectrum showed a tetra-coordinated boron peak, the chemical shift indicated that the boron monomer was bound by a DMSO molecule instead of phosphine. The ${ }^{31} \mathrm{P}$ NMR spectrum, meanwhile, showed a free unbound phosphine monomer. Both spectra suggested that phosphine monomer was replaced by DMSO in binding with the boron. The weak nature of the binding between these two monomers was an interesting result and suggests that this Lewis pair could potentially be used as a reversible crosslinker in producing polymeric networks. Since the related work is not a part of this project, however, only some early work is discussed briefly later in Section 3.4.

\subsubsection{Formation of Frustrated Lewis Pair using StPMes 2 and $\mathrm{StBPh}_{2}$ and their Binding with DEAD}

As stated above, the diphenyl-substituted phosphine monomer was able to bind to $\mathbf{S t B P h}_{2}$ due to the lower steric hindrance. In theory, the introduction of methyl groups on the ortho-position of aromatic rings in triarylphosphine should dramatically increase the steric hindrance around the phosphorus atom, thus prohibiting the formation of CLP. This was confirmed by mixing a 1:1 equivalent of StPMes2 and $\mathbf{S t B P h}_{2}$ in deuterated toluene. There was no change in the chemical shifts in either the

${ }^{11} \mathrm{~B}$ and ${ }^{31} \mathrm{P}$ NMR spectra, which confirmed the formation of a frustrated Lewis pair.

The obtained FLP was tested using different small molecules for activation, for example, butadiene, phenylisocyanate, benzaldehyde etc. This FLP did not show reactivity towards to these commonly studied molecules in FLP Chemistry due to the intermediate Lewis acidity of the boron-containing monomer, however. Finally, diethyl azodicarboxylate (DEAD) was selected as the trigger molecule for the gelation 
experiment. The binding of this molecule with our FLP system was tested using monomers directly. At high temperatures, however, side reaction was found to have occurred on the vinyl groups of the monomers, which was confirmed by monitoring the change in the relative integrals of the vinyl peaks in the ${ }^{1} \mathrm{H}$ NMR spectra. The side reaction could be between vinyl groups and the binding product or the selfpolymerisation of monomers. The former will change the binding pattern completely, and the latter will make detection of boron peaks difficult. Since the vinyl group would be consumed after polymerisation, it should not affect the reactivity of the resultant polymeric FLP. To investigate the binding of DEAD to our FLP system, two model molecules were synthesised, triphenylborane $\mathbf{B P h}_{3}$ and phenyldimesitylphosphine PhPMes2, each of which are analogues to the boron and phosphorus-monomers discussed previously. Figure 3.1 below, is a schematic representation of the binding between PhPMes2, BPh3 and DEAD, along with the NMR spectra of the reaction mixture.

An equivalent amount of PhPMes2, BPh3 and DEAD were mixed in deuterated toluene. The mixture was then tested by NMR spectroscopy at r.t. The ${ }^{1} \mathrm{H}$ NMR spectrum showed a noticeable broadening of peaks, which was probably due to the complicate conformational changes of the products caused by the steric hindrance around B-N-N-P centres. The activation of DEAD resulted in B-N-N-P linkage, which was previously reported by Maron and Bourissou et al. ${ }^{131} \mathrm{P}$ NMR showed several peaks after binding (Figure 3.1 (B)), but, among these, the unbound phosphine was still present, giving a peak at $-22.1 \mathrm{ppm}$ with an integral of only $1.2 \%$ in total. The mono-binding product of PhPMes2 and DEAD gave a sharp peak which was deshielded to $+25.8 \mathrm{ppm}$. The relative abundance of this mono-binding product was much higher, being $52.2 \%$ in total by integral. There were also another two peaks with much higher chemical shifts at +44.5 and +33.3 ppm respectively, which corresponded to the desired complex product PhPMes2 $\mathbf{D E A D} \cdot \mathbf{B P h}$. These two peaks were from 
the two possible isomers of PhPMes2-DEAD-BPh 3 , suggested in Figure 3.1 (A). The broad pattern of these two peaks indicated the complicated conformational changes of the two isomers, which is consistent with the ${ }^{1} \mathrm{H}$ NMR spectrum. The existence of multiple species in the reaction mixture indicated that the binding between PhPMes2-DEAD and BPh 3 was dynamic. This was also further confirmed by the ${ }^{11} \mathrm{~B}$ NMR spectrum, which gave only one, albeit very broad peak, at 6.0 ppm (Figure 3.1 (C)). No unbound boron was observed in the spectrum, probably because of the much slower timescale of the ${ }^{11} \mathrm{~B}$ NMR spectrometer compared to the binding exchange rate between $\mathbf{B P h}_{3}$ and the PhPMes2-DEAD mono-adduct. To investigate the dynamic nature of the binding further, a variable-temperature NMR experiment was performed for the product PhPMes2-DEAD-BPh 3 in the range between $300 \mathrm{~K}$ and $370 \mathrm{~K}$ with a $10 \mathrm{~K}$ interval. The results are shown below in Figure 3.2 and Figure 3.3.

(A)

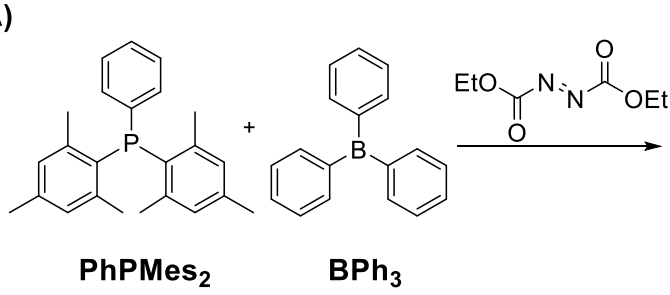

(B)
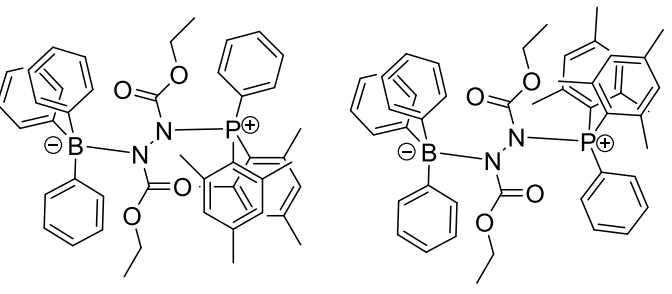

$\operatorname{PhPMes}_{2} \cdot \mathrm{DEAD} \cdot \mathrm{BPh}_{3}$

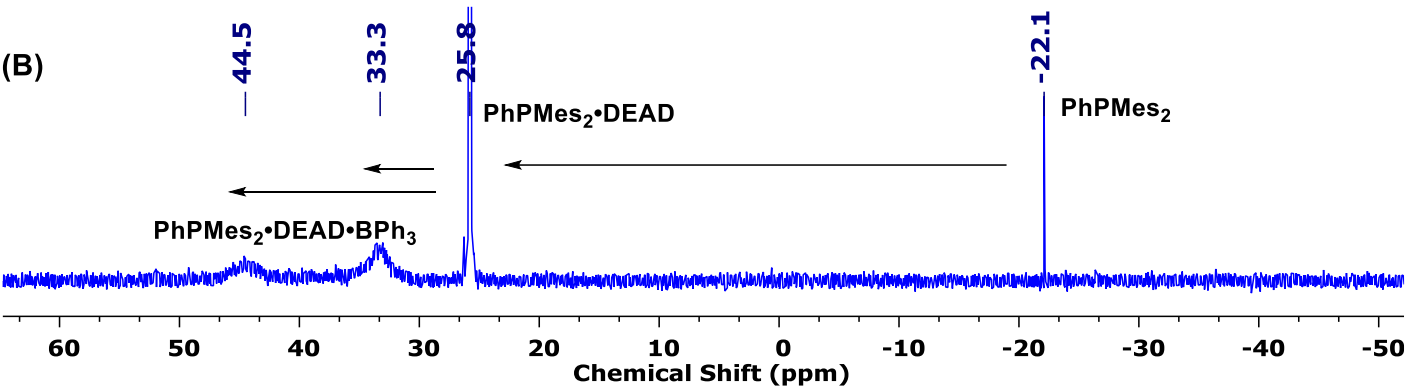

(C)

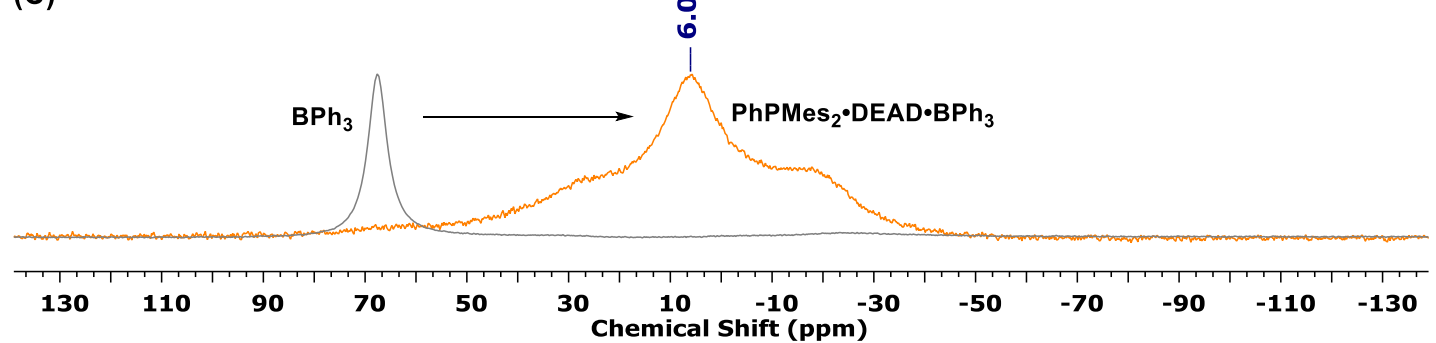

Figure 3.1 (A) Schematic representation of the binding of PhPMes2, BPh 3 and DEAD;

(B) ${ }^{31}$ P NMR spectrum of the binding experiment; (C) overlapped ${ }^{11} \mathrm{~B}$ NMR spectra of unbound $\mathbf{B P h} 3$ (grey line) and the binding experiment product (orange line). 


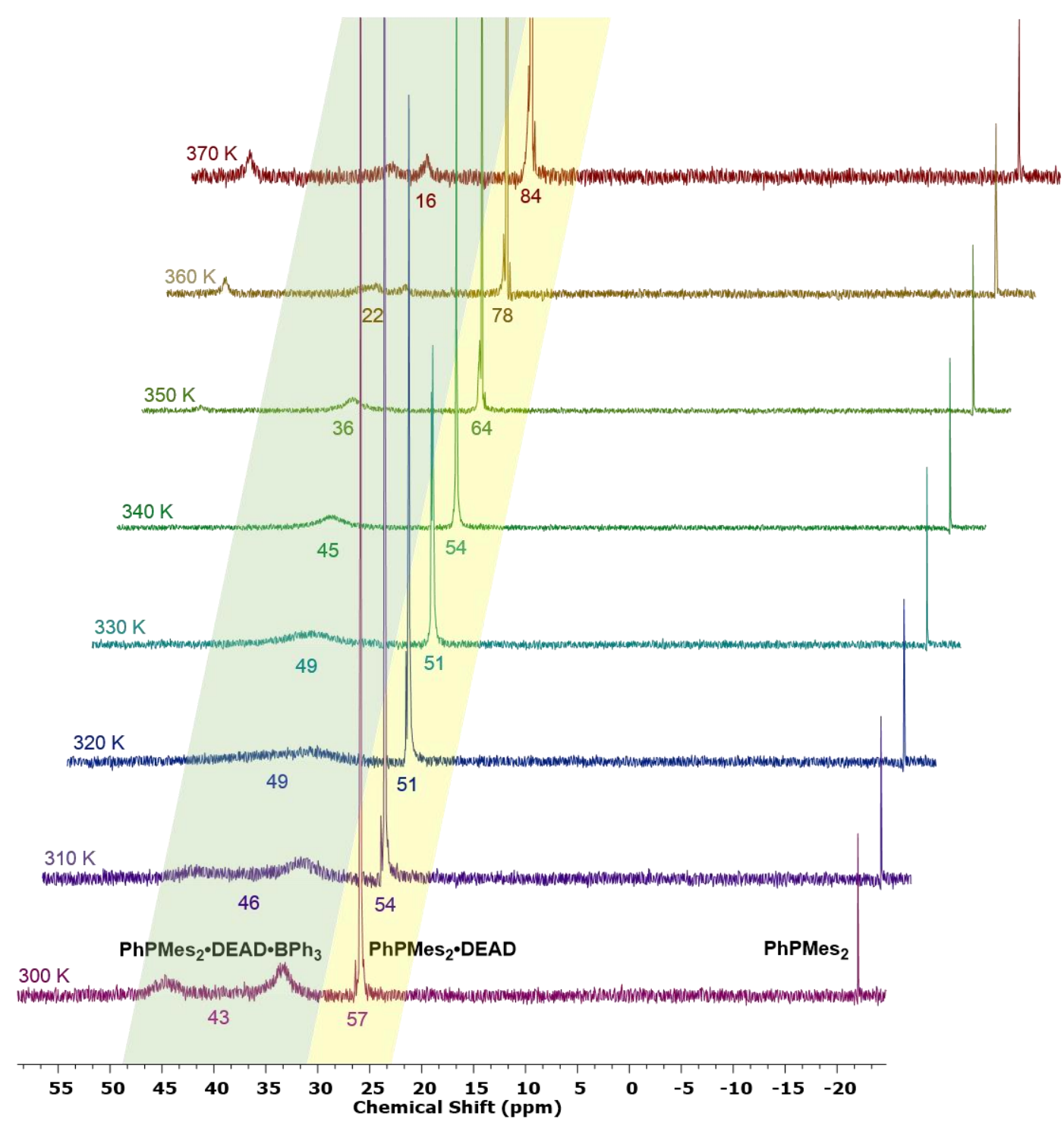

Figure 3.2 Variable-temperature ${ }^{31} \mathrm{P}$ NMR spectra of PhPMes2-DEAD $\cdot \mathbf{B P h} 3$. The numbers under each spectrum show the relative integrals of the peaks in the green and yellow area.

As shown in the ${ }^{31} \mathrm{P}$ NMR spectra (Figure 3.2) above, the first notable aspect of the change in the product with temperature is the faster exchange rate of its two isomeric forms. When the temperature increased to $330-340 \mathrm{~K}$, the two broad peaks corresponded to the isomers of $\mathbf{P h P M e s} 2 \cdot \mathbf{D E A D} \cdot \mathbf{B P h} \mathbf{h}_{3}$ recombined to give one broad peak at $38.3 \mathrm{ppm}$. The relative integration of $\mathbf{P h P M e s} 2 \cdot \mathbf{D E A D} \cdot \mathbf{B P h} \mathbf{H}_{3}$ remained 
constant at around $48 \%$ in total below $350 \mathrm{~K}$, indicating that the isomeric exchange was faster compared to the escape of $\mathbf{B P h}$. The amount of this complex product started to decrease, however, when the temperature went above $350 \mathrm{~K}$, and at the same time, some unidentified by-product formed to give some small peaks around $53.2 \mathrm{ppm}$. The relative amount of PhPMes2-DEAD-BPh 3 and PhPMes2-DEAD species changed from $43: 57$ at $300 \mathrm{~K}$ to $49: 51$ at $320 \mathrm{~K}$, and finally to $16: 84$ at $370 \mathrm{~K}$, which confirmed that the binding between PhPMes2-DEAD mono-adduct and $\mathbf{B P h} 3$ is a dynamic process and responsive to temperature.

The ${ }^{11} \mathrm{~B}$ NMR spectra at variable temperatures also led to similar conclusions. As shown in Figure 3.3 below, at lower temperatures, from $300 \mathrm{~K}$ to $330 \mathrm{~K}$, there was only one very broad peak, which was the binding product PhPMes2 $\cdot$ DEAD $\cdot$ BPh3. When the temperature increased to $340-350 \mathrm{~K}$, however, this peak became much sharper than was observed previously and appeared at a slightly lower chemical shift. It is proposed that this was a result of stronger binding between boron and the PhPMes2-DEAD mono-adduct due to the faster isomeric exchange. When the temperature was increased to $360 \mathrm{~K}$, however, a broad peak started to appear with a much higher chemical shift; and at $370 \mathrm{~K}$, a peak at $64.7 \mathrm{ppm}$ was evident, which corresponded to the released boron compound $\mathbf{B P h}$.

Both the ${ }^{11} \mathrm{~B}$ and ${ }^{31} \mathrm{P}$ NMR spectra, therefore, showed the dynamic nature of binding between $\mathbf{B P h}_{3}$ and the PhPMes2 - DEAD mono-adduct. This promising result indicated that our FLP system could be used to generate a polymer gel with dynamic crosslinking. The phase transition temperature was determined as $350-360 \mathrm{~K}$. This was expected to change, however, when the system was incorporated into macromolecules. One thing that needs to be considered is that the dynamic nature of the binding depends greatly on the solvent system. When a poor solvent was used, like chloroform, the complex product PhPMes2-DEAD-BPh3 could barely dissolve, which served to shift the 
reaction equilibrium to the direction of its formation. As a result, almost no PhPMes2-DEAD mono-adduct could be seen in the ${ }^{31} \mathrm{P}$ NMR spectrum, indicating no, or very slow exchange between it and the boron. For the later gelation experiments, toluene was selected as the solvent to dissolve polymers, and, more importantly, to enable the dynamic exchanges between FLP-DEAD crosslinks.

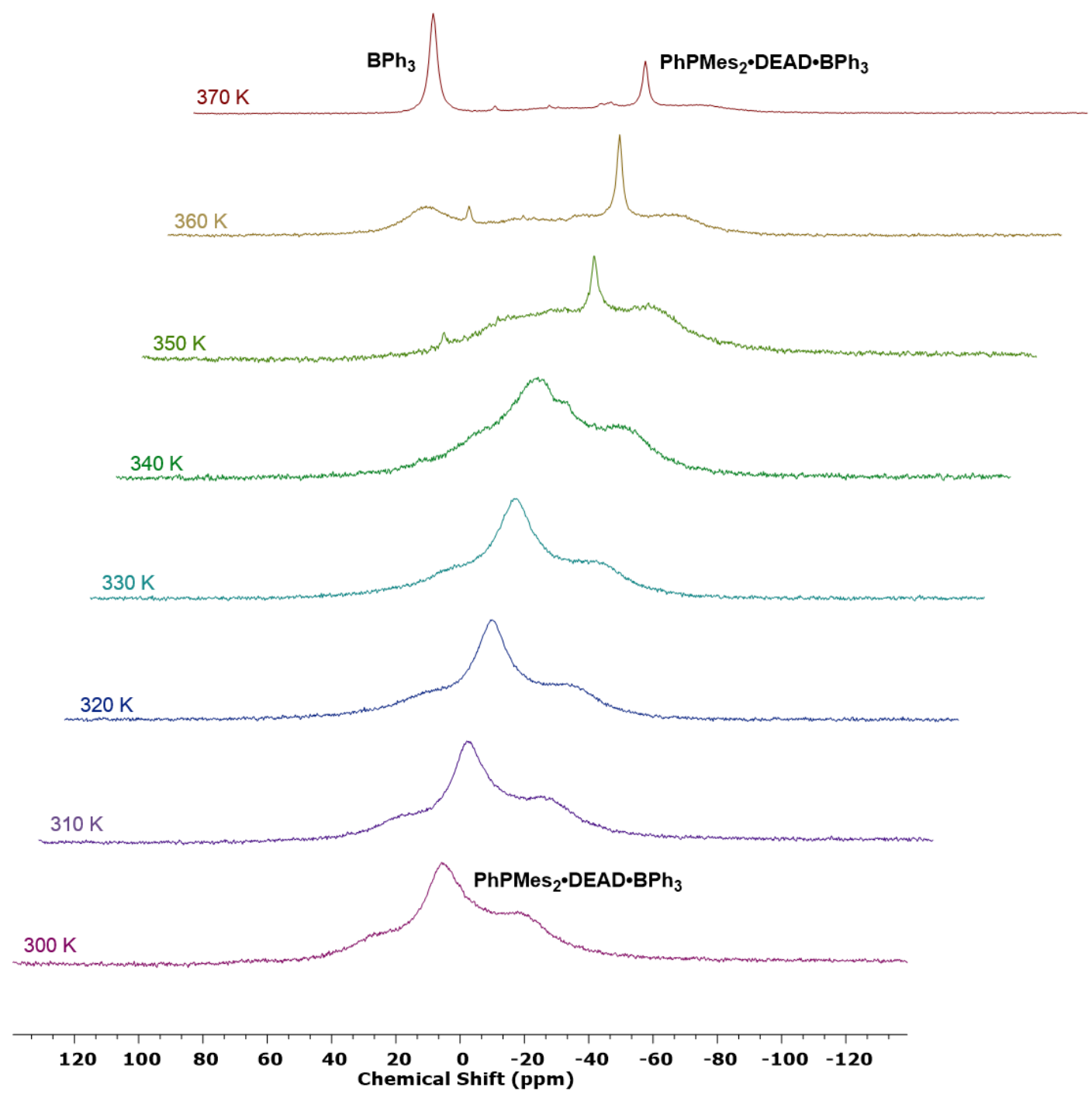

Figure 3.3 Variable-temperature ${ }^{11}$ B NMR spectra of PhPMes2 $\cdot$ DEAD·BPh3. 


\subsection{Gelation Experiments}

With both phosphorus and boron containing polymers in hand, the gelation experiment was performed. Different combinations of copolymers with various boron/phosphorus contents were used for gel formation, including P1B1, P1B2, P1B3, P2B2 and P3B3. For all samples, the total concentration of polymer chains was kept the same, and the amount of phosphine and boron moieties was maintained at a 1:1 equivalent. The reaction is shown below in Scheme 3. 2 .

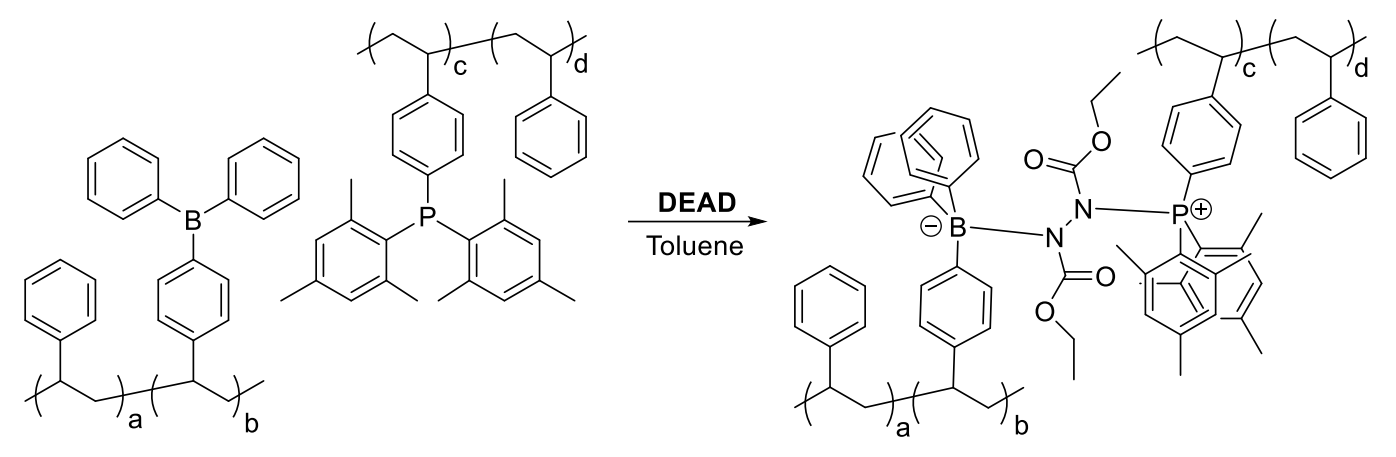

Scheme 3. 2 Synthesis of poly(FLP) network.

Gelation using copolymers P1 (6.7 \% phosphorus) and B1 (7.5 \% boron) is shown below in Figure 3.4. The two copolymers were dissolved in toluene and mixed. Since the boron and phosphorus units form an FLP, they cannot bind together and hence their copolymers remained as single chain polymers and soluble in the solvent. Six equivalents of DEAD were quickly injected into the solution. The excess amount of DEAD ensured the shift of reaction equilibrium towards to complete binding. Once the DEAD spread into the polymer solution, it immediately reacted with the surrounding boron and phosphorus moieties. This resulted in the formation of crosslinks between polymer chains. The dramatic increase in molecular weight and the generation of a complicated three-dimensional structure meant that the polymers were no longer be able to dissolve, hence precipitate out of the solvent. The gelation process was speedy, and within about 2 seconds of the injection of the DEAD, the solution started to become non-transparent, which is the indication of crosslinking. After about 
10 seconds, the polymer network became strong enough to stick to the bottom of an inverted sample vial without falling.

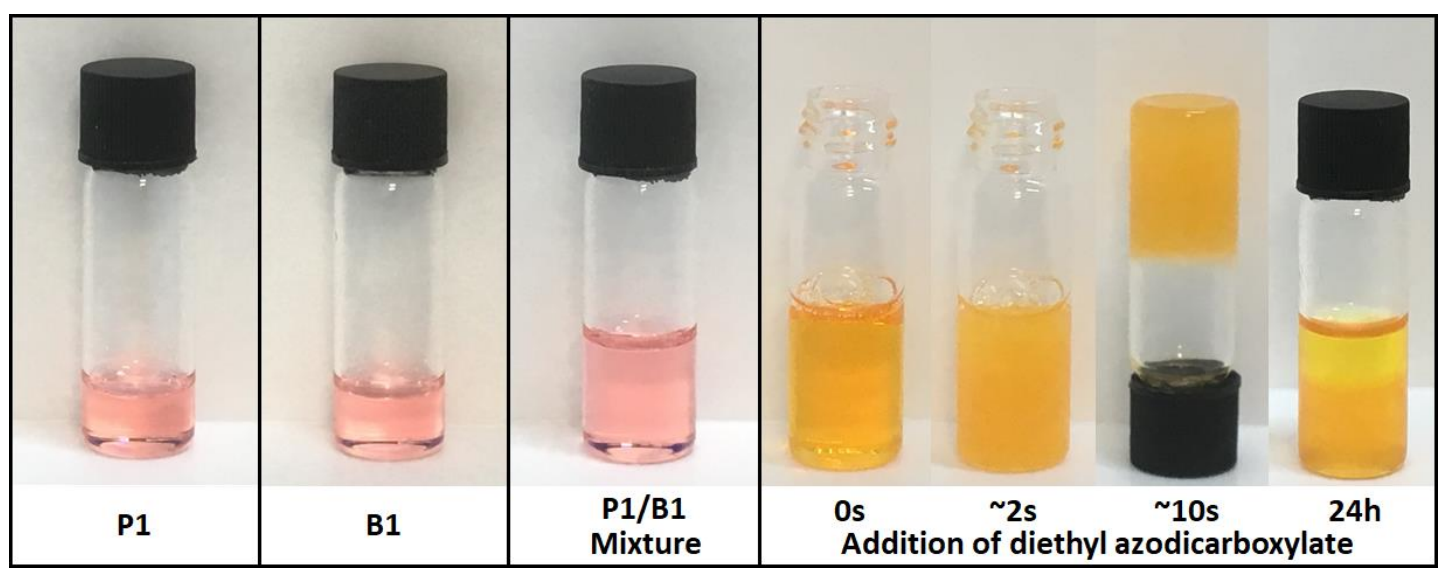

Figure 3.4 Gelation experiment using Copolymers P1 and B1, and DEAD.

Some shrinkage was observed in the volume of the produced polymer gel P1B1 after crosslinking, and some solvent was squeezed out on top of the gel, as shown in Figure 3.4. To investigate this gel shrinkage, the volume of the polymer gel was continually measured after the DEAD injection. The change in gel volume vs. time was plotted in the graph below in Figure 3.5.

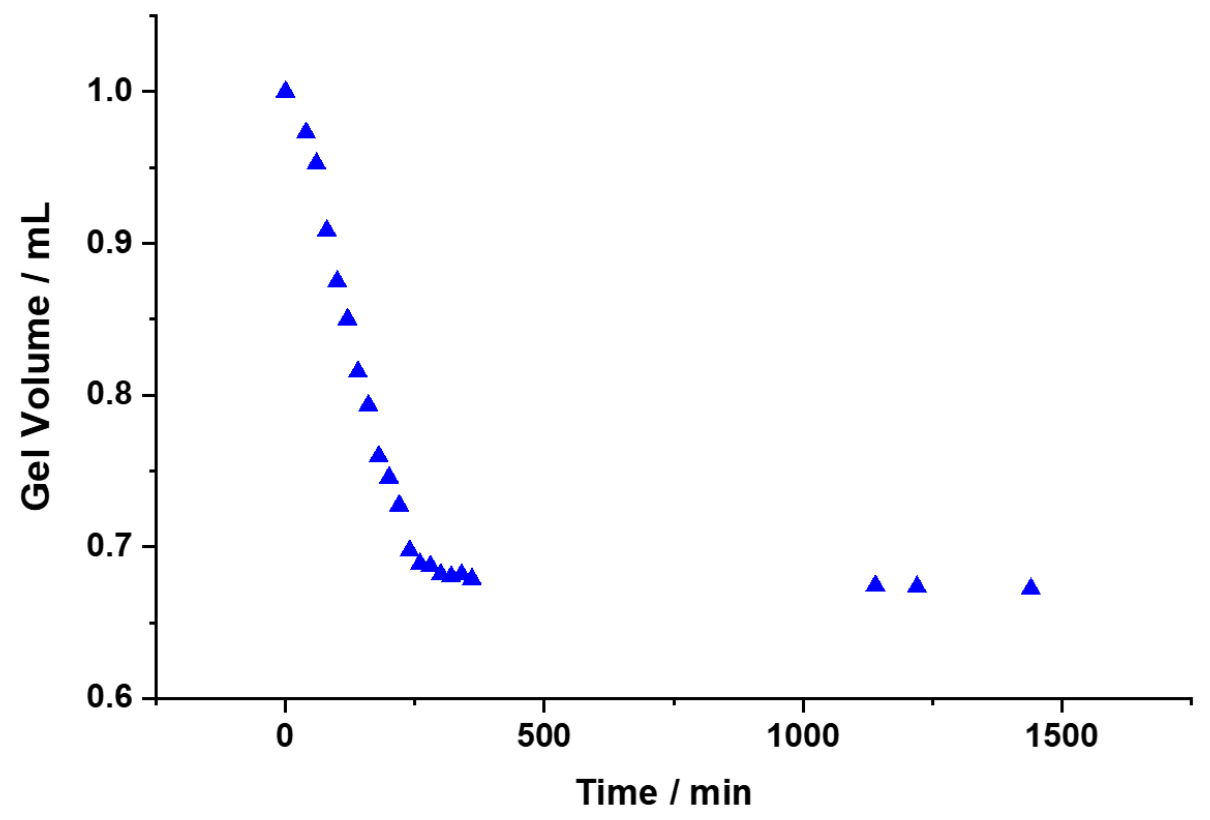

Figure 3.5 Shrinkage in the volume of the P1B1 gel after injection of DEAD. 
It can be seen that a rapid decrease in the gel volume occurred during the first $240 \mathrm{~min}$ after the injection of DEAD, but that the volume stabilised thereafter at about $67 \%$ of its initial volume. This reduction in the gel volume could be attributed to the crosslinking exchange. Once the DEAD was added, a large amount of crosslinks were formed immediately, which dramatically increased the local constraints, making it difficult for the polymer chains to arrange themselves in the space available, hence forming a temporary set gel. After this initial rapid gelation, however, the FLPcrosslink continue to equilibrate due to its dynamic nature. This allowed the polymer chains to get some freedom to diffuse slowly, which led to the formation of additional crosslinks. As a result, a more stable gel was formed with a smaller volume.

Other polymer gels were also synthesised in a similar way. Gel P1B2 $(6.7 \%$ phosphorus and $15.9 \%$ boron) also exhibited a reduction in volume after DEAD injection. In this case, a much longer time (over $500 \mathrm{~min}$ ) was required for complete shrinking (59\%). It seemed that when the boron concentration is higher, a more constraint gel is formed during the initial rapid gelation period, probably because the formation of the crosslinks between individual macromolecules is more favourable compared to that of extended networks. This presumption was confirmed by gel P1B3, which was also highly constrained, making reorganisation impossible. Thus no shrinkage was observed, even after $48 \mathrm{~h}$. Due to the lack of reorganisation, the strength of P1B3 was severely limited, and the gel broke very easily.

Attempts were also made to form gel P2B2 (13.3\% phosphorus and $15.9 \%$ boron) and P3B3 (22.9 \% phosphorus and $29.9 \%$ boron), but in these cases no homogeneous gel could be obtained, as shown below in Figure 3.6. The addition of DEAD into the mixtures of highly abundant phosphorus- and boron-containing copolymers only resulted in rapid precipitation of crosslinked clusters. This can also be explained by 
the previous presumption, that a high concentration of FLP seriously limited the reorganisation of polymer chains. The compromised crosslinking exchange cannot afford the connection of discrete regions. Hence the synthesis only gave small clusters.

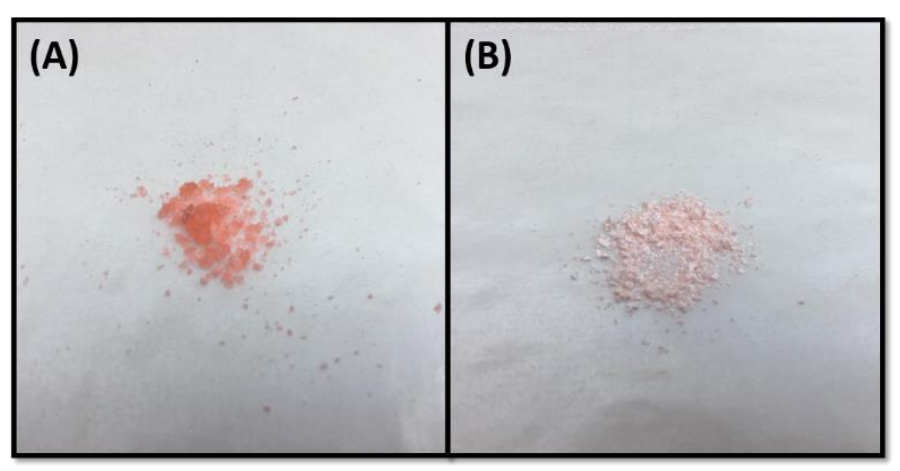

Figure 3.6 Products of the attempted synthesis of gel (A) P2B2 and (B) P3B3.

\subsection{Characterisation of Poly(FLP) Gel}

\subsubsection{Infrared Spectroscopy}

Although the formation of the gel can be observed visually, an analytical method was also performed to characterise it. The first method attempted was the use of NMR spectroscopy. It was found to be very difficult to prepare the gel inside a narrow NMR tube, however. When DEAD was injected, the gel could only form on the top surface inside the NMR tube, and this prevented the diffusion of DEAD to the bottom, leading to incomplete gelation. Precipitated gels are also unlikely to give reasonable signals for liquid phase NMR spectroscopy. Infrared (IR) spectroscopy was therefore used instead, as shown below in Figure 3.7. 


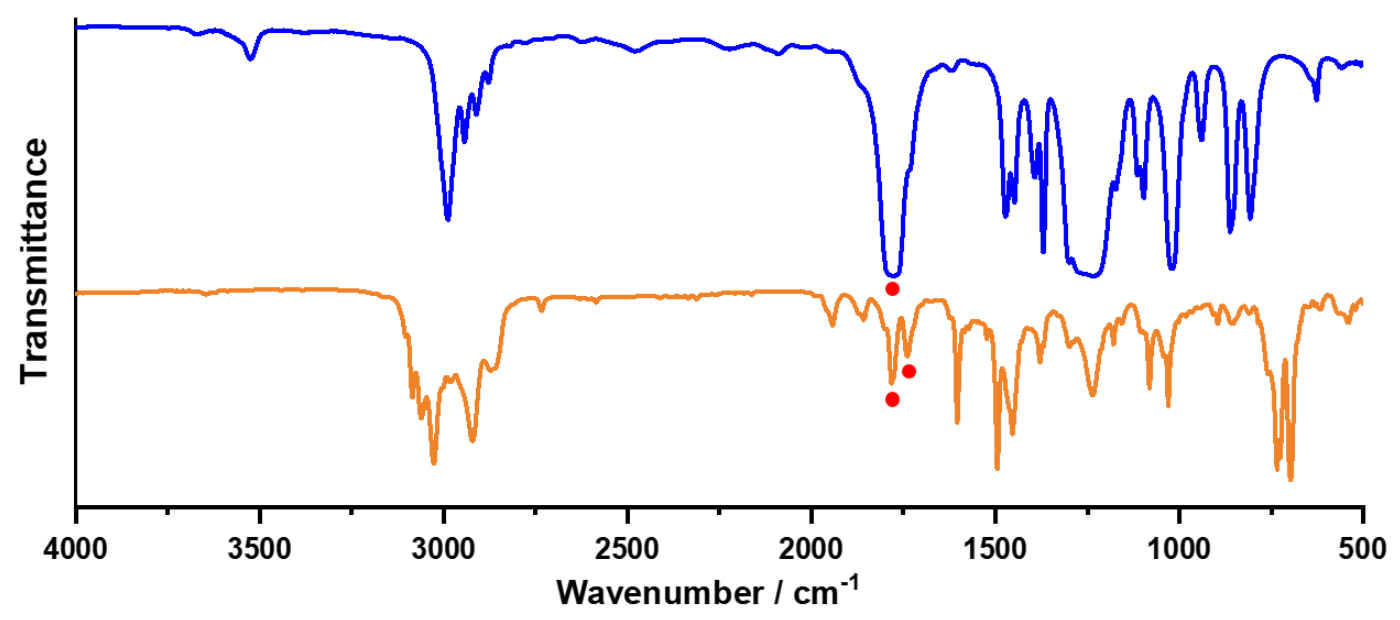

Figure 3.7 IR spectra of DEAD (top) and poly(FLP) gel P1B1 (bottom). The carbonyl bands are denoted with red dots.

The lack of carbonyl groups in the non-crosslinked polymers enabled the characterisation of the DEAD in the polymer gel by IR spectroscopy. As shown above, the pure DEAD only gave one stretching $\mathrm{C}=\mathrm{O}$ band at $1777 \mathrm{~cm}^{-1}$ due to its symmetrical structure. When this was bound with boron and phosphorous, however, the molecule lost its symmetrical geometry. The resultant symmetrical and asymmetrical $\mathrm{C}=\mathrm{O}$ stretching of DEAD in the crosslinks were IR active and showed two bands at 1783 $\mathrm{cm}^{-1}$ and $1741 \mathrm{~cm}^{-1}$, respectively. The change in the stretching modes of DEAD before and after crosslinking proved its binding with the boron and phosphorus moieties.

\subsubsection{Swelling Ratio Measurement}

The ability to adsorb solvents without dissolving is a typical behaviour of polymer networks. ${ }^{2}$ In this regard, the swelling ratio of a polymer gel is defined as follows:

$$
\text { Swelling } \%=\frac{m_{t}-m_{0}}{m_{0}} \times 100 \%
$$

Where $m_{\mathrm{t}}$ is the mass of swollen gel at time $\mathrm{t}$, and $m_{0}$ is the mass of a gel before swelling. To investigate the swelling process of poly(FLP) gel, P1B1 was immersed in toluene and its mass was measured at specific time intervals. The results are shown in Figure 
3.8.

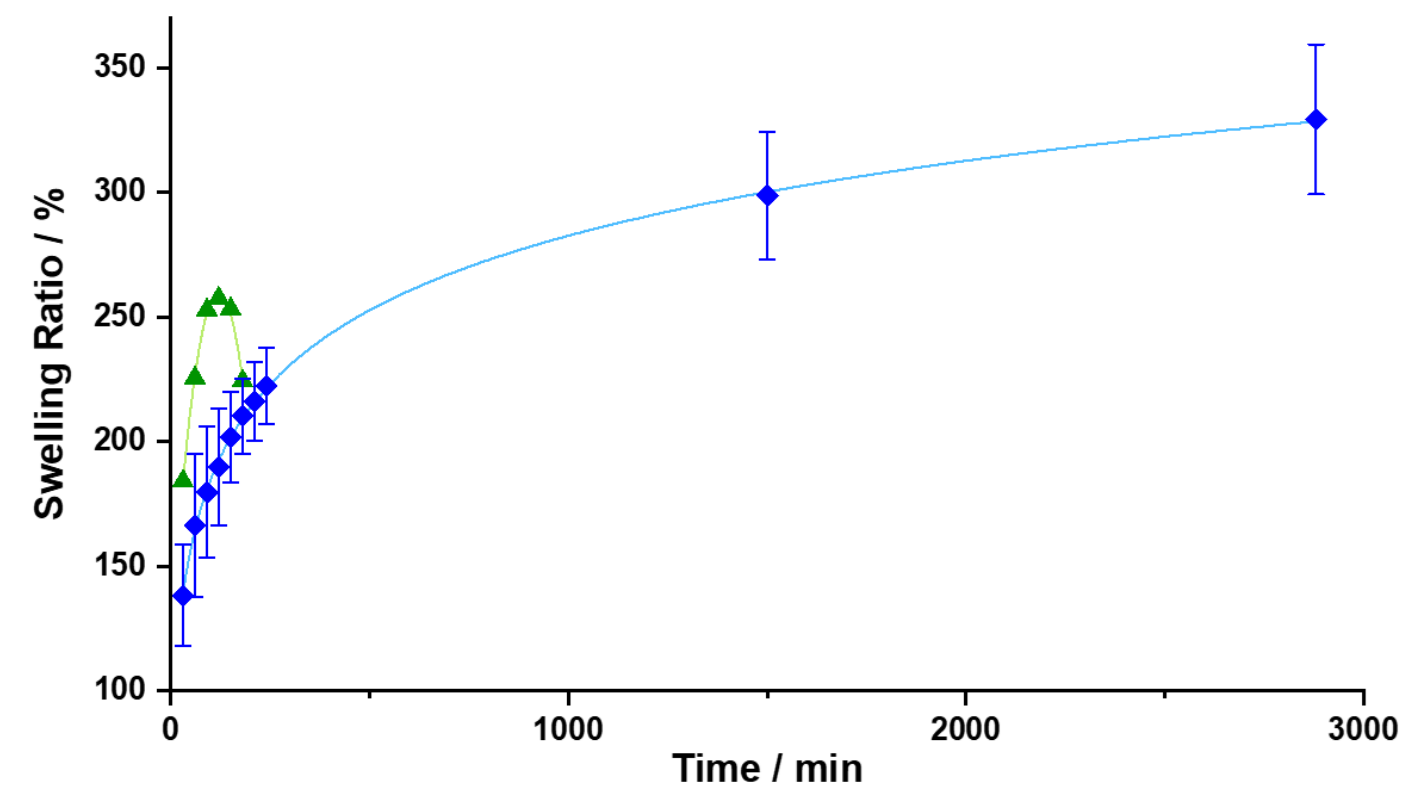

Figure 3.8 Swelling ratio of gel P1B1 (blue) and P1B3 (green).

All measurements were made three times, and an average was calculated for the graph. As shown above, the poly(FLP) gel showed the typical swelling behaviour of a polymeric network. The swelling was very fast initially, but then became much slower after the gel began to get saturated. The swelling ratio of P1B1 after $48 \mathrm{~h}$ reached $329 \%$ on average. P1B2 also exhibited similar behaviour, but not P1B3. Initially, the swelling of P1B3 was much faster than P1B1, but a dramatic decrease in gel mass occurred after $120 \mathrm{~min}$, which was caused by the cracking of the gel. This indicated that P1B3 was a much weaker gel compared to others, which was consistent with the hypothesis made in previous sections.

\subsubsection{Scanning Electron Microscopy}

A scanning electron microscopy (SEM) was used to probe the internal structures of the gels formed. Preparation of the gels P1B1, P1B2 and P1B3 for SEM study was 
attempted by using toluene as the solvent first. However, the very low vapour-pressure of toluene cannot afford the quick freeze-drying of this solvent under vacuum without harming the internal structure of the gels. Therefore, instead, benzene was used to prepare the gels. Freeze-drying was successfully applied to the resultant gels. The samples obtained were coated in gold and sent for SEM testing. The images obtained are shown below in Figure 3.9. To best of our knowledge, this is the first example of using benzene as the solvent to prepare SEM samples.
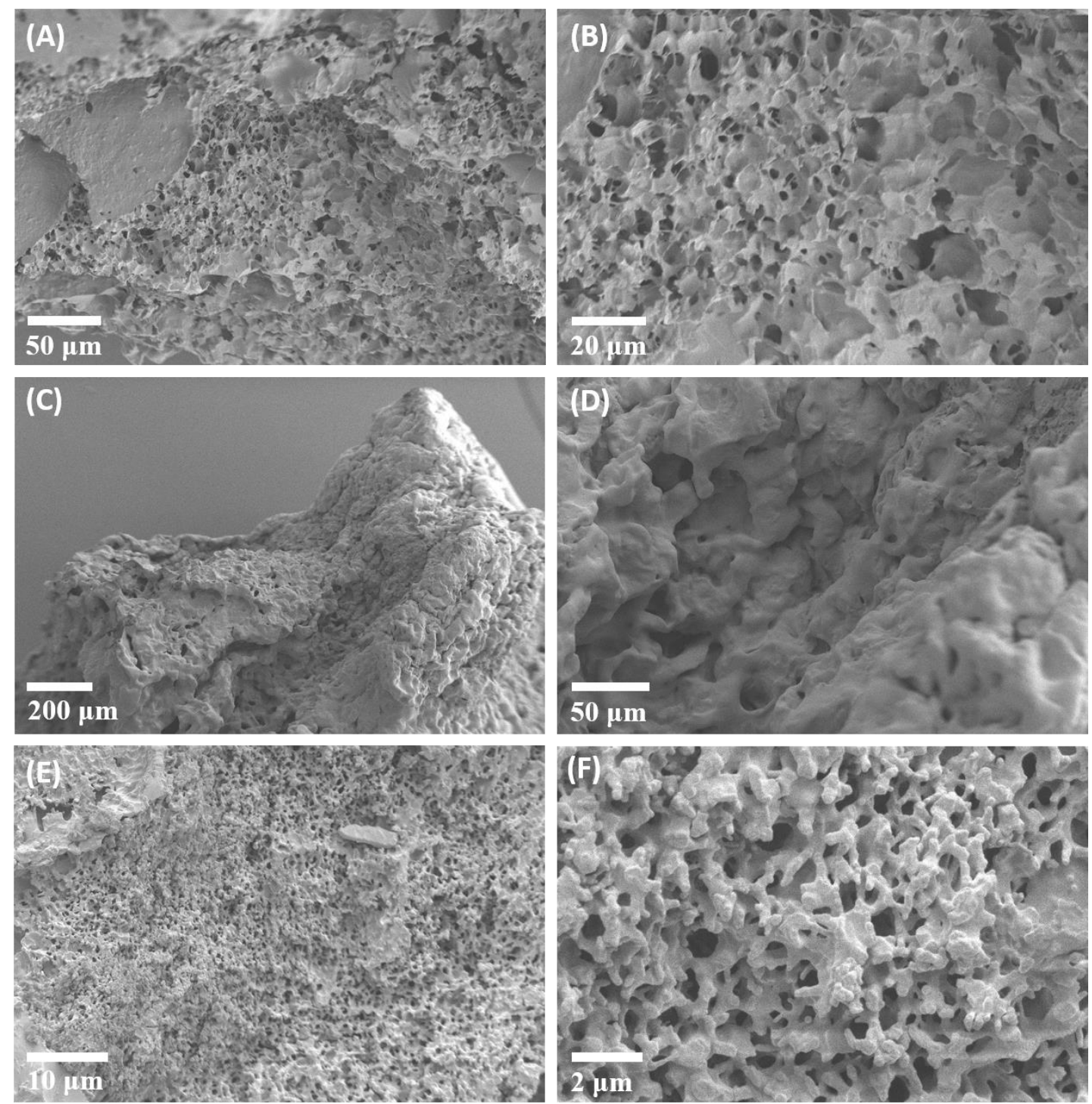

Figure 3.9 SEM images of poly(FLP) gels (A) P1B1; (B) higher magnification of P1B1; (C) P1B2; (D) higher magnification of P1B2; (E) P1B3; (F) higher magnification of P1B3. 
The SEM images for all the samples clearly showed the existence of pores and filaments, which is the typical structure of a polymer network. Gel P1B1 showed a condensed structure with relatively strong polymer filaments. The sizes of both the filaments and pores were irregular, although the pores were relatively small in size compared to the polymer filaments. The pores were distributed over the polymer network. Gel P1B2 exhibited an entirely different, mainly porous and compact continuous structure. Gel P1B3, meanwhile, had a very regular structure of pores and filaments, with diameters of about 500-1000 nm and 300-600 $\mathrm{nm}$ respectively. The lack of a continuous phase of polymer composite, and the relatively small size of the polymer filaments, however, suggested that this gel would be weak, and this indeed was consistent with the experimental results obtained from gel preparation and swelling ratio measurements.

\subsubsection{Rheology}

Rheology measures the deformation and flow of matter under stress. It can be used to measure, not only liquids, but also some viscoelastic solid materials like polymers. ${ }^{3}$ Rheology was ascertained by loading samples between two plates, where the bottom one was fixed in place while the top one exerted forces onto the samples, as shown below in Figure 3.10. The plates have different shapes and surfaces to stop the encased material sliding, since this would lead to inaccurate results. In this study, a crosshatched plate was used at the bottom as the stationary plate, and a rough-surfaced plate was used on top as the oscillating plate. All the measurements in respect to the poly(FLP) gels were performed under air since the gel gains some air-stability due to the coordination of boron by DEAD. The gel was confirmed to be able to keep in shape within at least seven days of exposure to air. Although shearing may lead to faster degradation, this did not seem to happen during the testing, as the highly reproducible rheology results can be obtained. The gel was measured in its fully swollen state with 
toluene as the solvent. To prevent any evaporation of solvent, the samples were covered with an inert fluorinated oil. The key mechanical properties of poly(FLP) gels were measured by different rheology testing methods, which are discussed as follows.

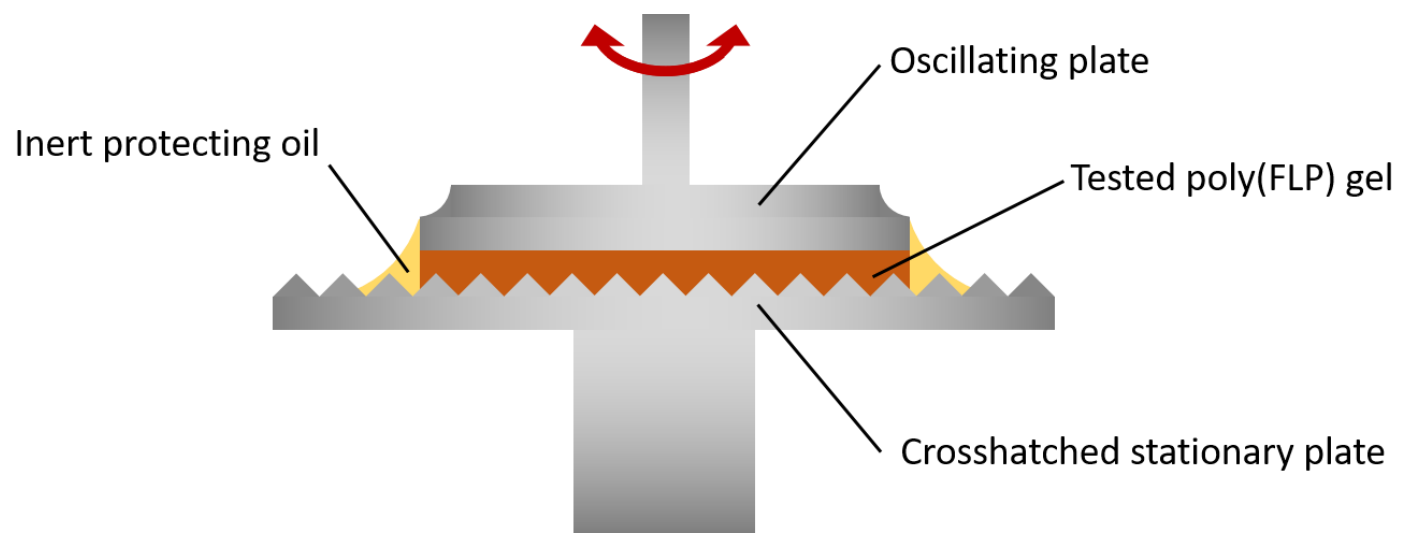

Figure 3.10 Graph to show the rheology method used in this project.

\subsubsection{Dynamic Nature of Crosslinking}

To investigate the dynamic nature of crosslinks, a frequency-sweep test was performed on the gel P1B1. In this test, the sample was deformed by oscillating shear stress with deformation to a $0.1 \%$ strain. The result is shown below in Figure 3.11.

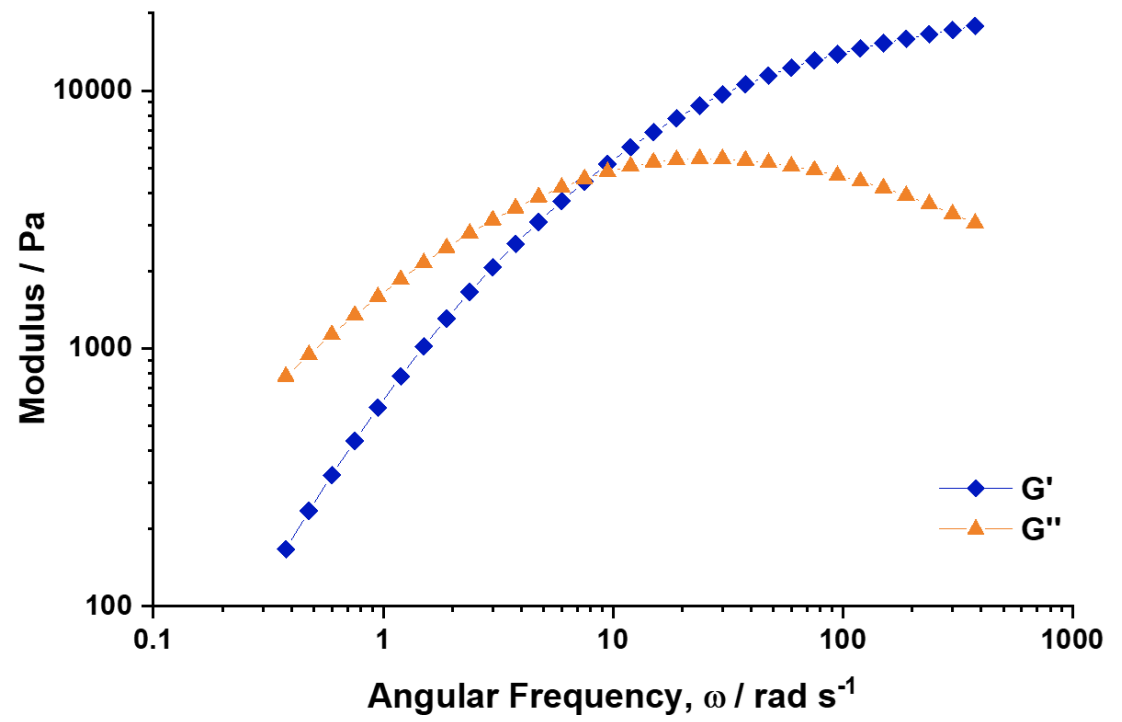

Figure 3.11 Frequency-sweep test of gel P1B1 at $0.1 \%$ strain, $22{ }^{\circ} \mathrm{C}$. 
$\mathrm{G}^{\prime}$ is the storage modulus, which is a measure of the energy stored in the material when a force is applied to it, and G" is the loss modulus, which is a measure of energy dissipated as heat when deformation is applied. ${ }^{3-4}$ Hence G' and G" represent, respectively, the elastic and viscous responses of material. ${ }^{3-4}$ Both moduli are important components of the overall modulus of a material. For a solid material, since the elastic behaviour is predominant, the storage modulus is always higher than the loss modulus. In contrast, the loss modulus for a liquid is always higher than the storage modulus. As shown in the figure above, the poly(FLP) showed completely different behaviour to conventional solids or liquids. At low angular frequencies, the loss modulus was predominant. Hence the material behaved more like a liquid and relaxed over a long timescale. When the angular frequency increased up, the storage modulus started to increase and finally became higher than the loss modulus. The crossover point was at $9.5 \mathrm{rad} / \mathrm{s}$ in this case. This behaviour has previously been observed in some dynamic covalent and non-covalent crosslinked polymer networks at the temperature of crosslinking exchange. ${ }^{5-8}$ This result, therefore, suggested that the crosslinks of the poly(FLP) gel system were exchangeable at room temperature, which is consistent with the NMR binding experiments using boron-, phosphorusmodel compounds and DEAD, as stated before.

\subsubsection{Time-Temperature Superposition}

Dynamic crosslinked systems often have crossover points of G' and G' that shift with temperature since the crosslinking exchange rate is temperature dependent. As the one who was trained with the rheometer in our group, Utku Yolsal performed this and the subsequent rheology tests. As shown below in Figure 3.12, the frequency-sweep tests were performed at $0,10,20,30,40$ and $50{ }^{\circ} \mathrm{C}$ with $1 \%$ strain. The temperature was limited to $50{ }^{\circ} \mathrm{C}$ to avoid evaporation of solvent. When the temperature was lowered down to $0{ }^{\circ} \mathrm{C}$, however, a crossover point with smaller angular frequency was observed, 
indicating that while the crosslinking exchange can still occur, it slows at this temperature. Due to the limitation of the testing machine, the temperature for our rheology tests could not be reduced below $0{ }^{\circ} \mathrm{C}$, however, it was believed a crossover point with even lower angular frequency could be observed if a much lower temperature was applied. Experimentally, a dramatic increase in the gel strength was observed visually if the gel was stored at $-30{ }^{\circ} \mathrm{C}$. When the temperature increased, there was a noticeable trend of the crossover points increasing in angular frequency. Moreover, in the case of $40^{\circ} \mathrm{C}$ and $50^{\circ} \mathrm{C}$, the crossover point was no longer observable within the range of testing frequency. This indicated faster crosslinking exchange and enhanced viscous behaviour of the gel at a higher temperature.
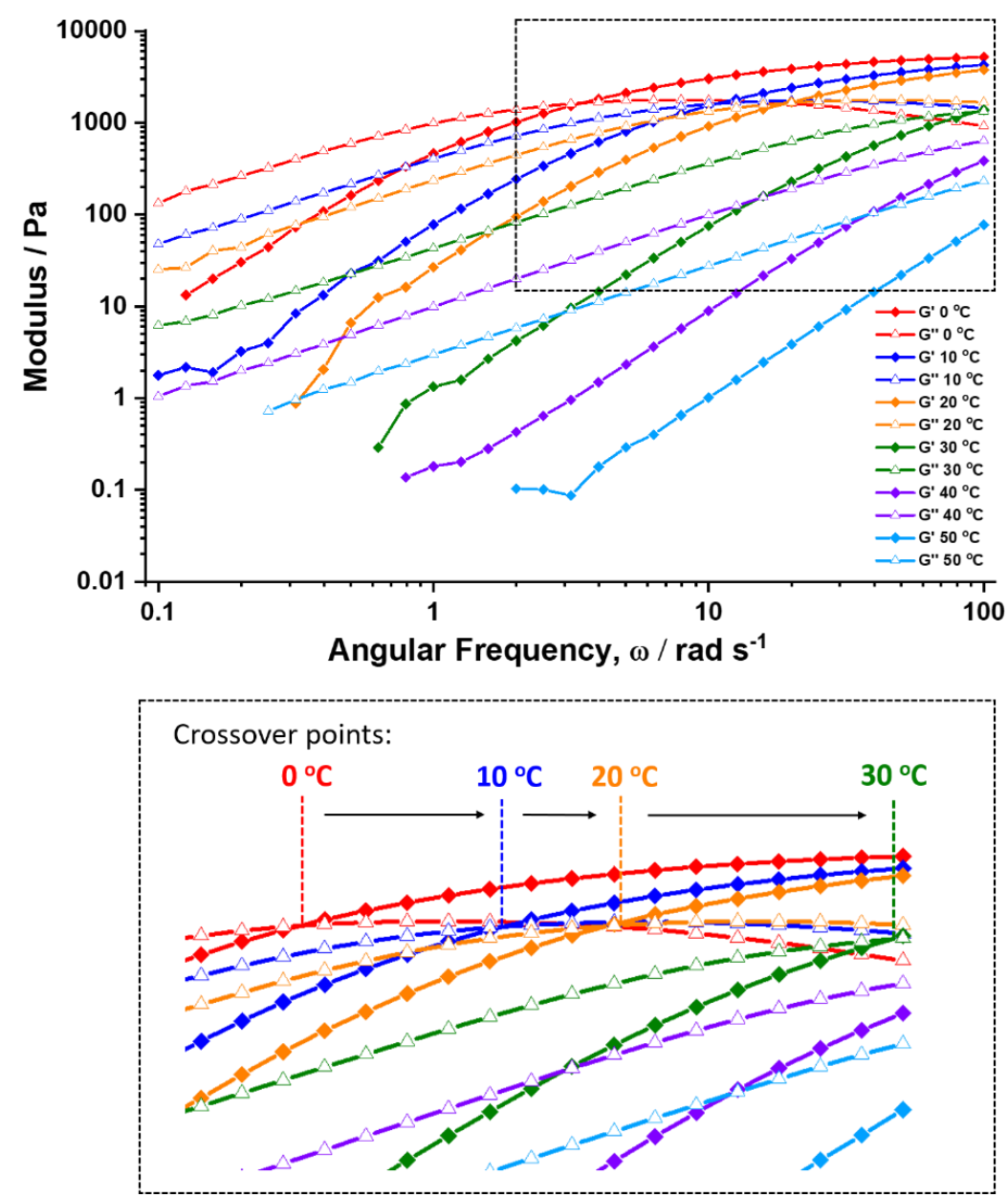

Figure 3.12 Overlapped frequency-sweep curves of poly(FLP) gel at different temperatures. 
Another finding from these frequency-sweep curves is the time-temperature dependence of our system. Using $20{ }^{\circ} \mathrm{C}$ as a reference, by overlapping the frequencysweep curves at different temperatures with a shifting factor $\mathrm{a}_{\mathrm{T}},{ }^{9}$ a master curve was obtained, which is known as the time-temperature superposition (TTS) curve, ${ }^{9-10}$ shown below in Figure 3.13. Successful plotting of the TTS curve suggested that the relaxation time of polymer chains in the poly(FLP) gel was the only physical parameter that was affected by temperature. This indicated that the poly(FLP) gel synthesised in this project was a class of supramolecular polymer network, which also has a typical behaviour of time-temperature dependence. ${ }^{11-12}$

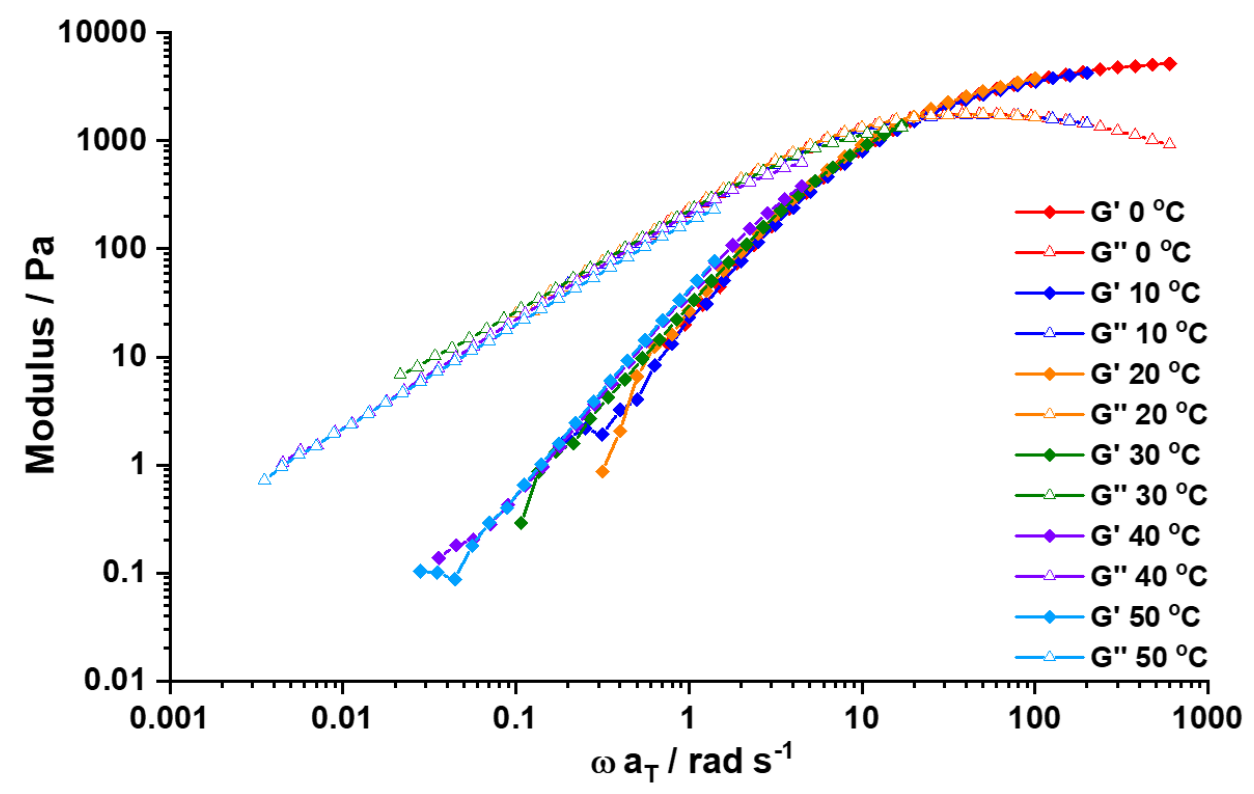

Figure 3.13 Time-temperature superposition graph of poly(FLP) gel.

\subsubsection{Temperature Responsiveness}

By the results obtained from the NMR binding test and frequency-sweep rheology, it was evident that the poly(FLP) gel can respond to temperature. A simple test was performed to verify this, as shown below in Figure 3.14. 


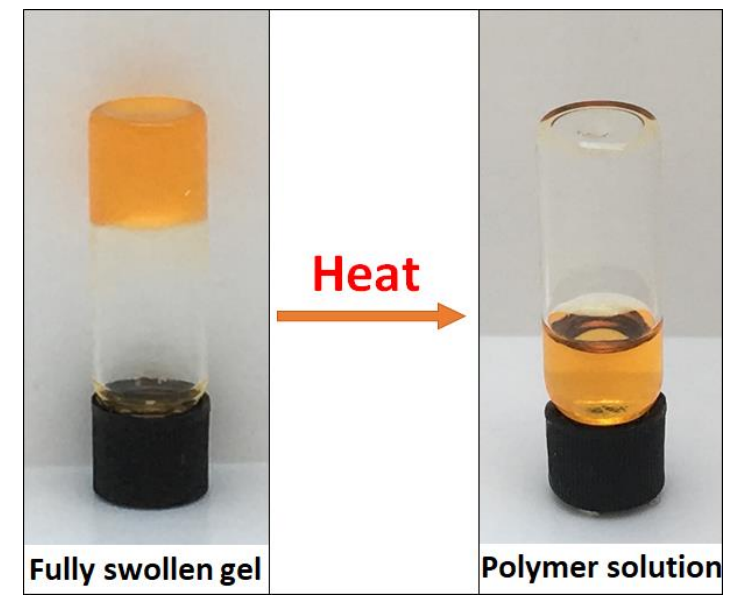

Figure 3.14 Rupture of poly(FLP) gel by heating.

A fully swollen gel P1B1 was prepared in a small sample vial, and heat was applied. At high temperature, the cleavage of FLP crosslinking was initiated. This caused the polymer chains to redissolve into the solvent, and as a result, the swollen gel reverted to the polymer solution.

The temperature responsiveness of poly(FLP) gel was also investigated by the temperature-ramp rheology test. The strain and heating rate was set to be $1 \%$ and $0.05^{\circ} \mathrm{C} / \mathrm{s}$, respectively. The results are shown below in Figure 3.15.

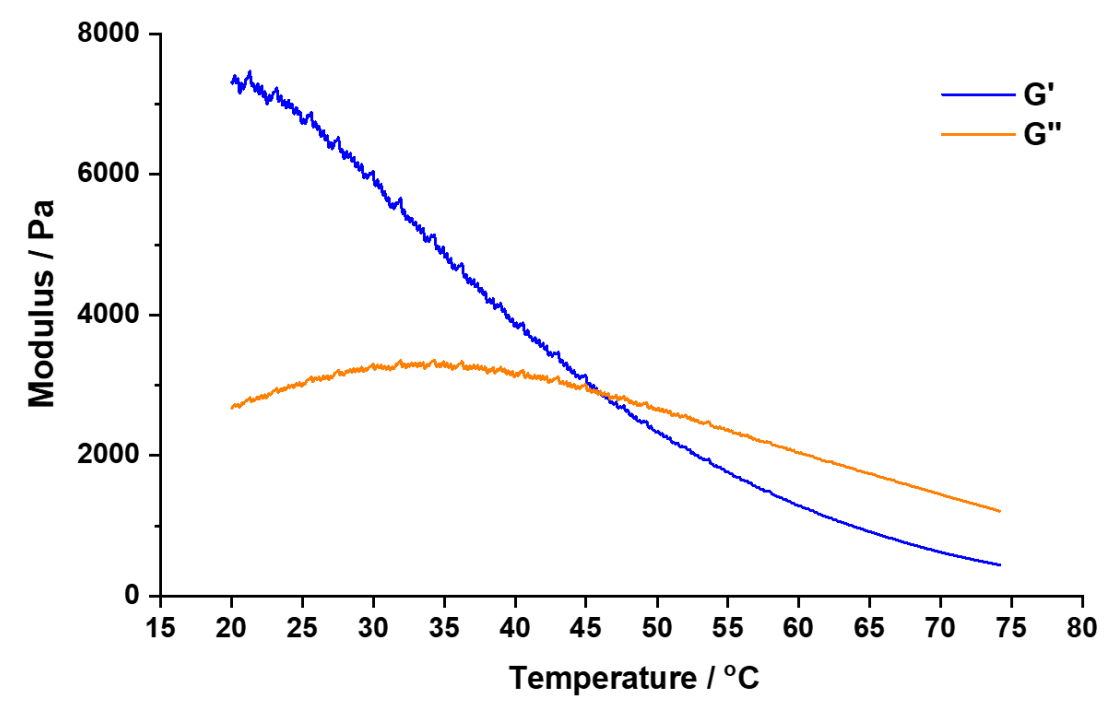

Figure 3.15 Temperature-ramp rheology test for poly(FLP) gel. 
It can be seen that both $G$ ' and $G$ " showed a trend to decrease with elevated temperature, although there was a hump for $\mathrm{G}$ " near $35^{\circ} \mathrm{C}$. A crossover point appeared at $45{ }^{\circ} \mathrm{C}$, which was the phase transition temperature for the material from a viscoelastic gel to viscous liquid. Although the crossover temperature may deviate from the real phase transition temperature, since it may be affected by many parameters such as strain, stress, etc., this temperature-ramp curve still intuitively shows the temperature responsiveness of the poly(FLP) gel.

\subsubsection{Self-healing}

From the testing results stated in previous sections, it was evident that the FLP crosslinks in the gel should be dynamic and exchangeable at room temperature, meaning that the material should be able to self-heal. To confirm this, self-healing experiments were performed, initially using completely dried poly(FLP) gel, as shown below in Figure 3.16.

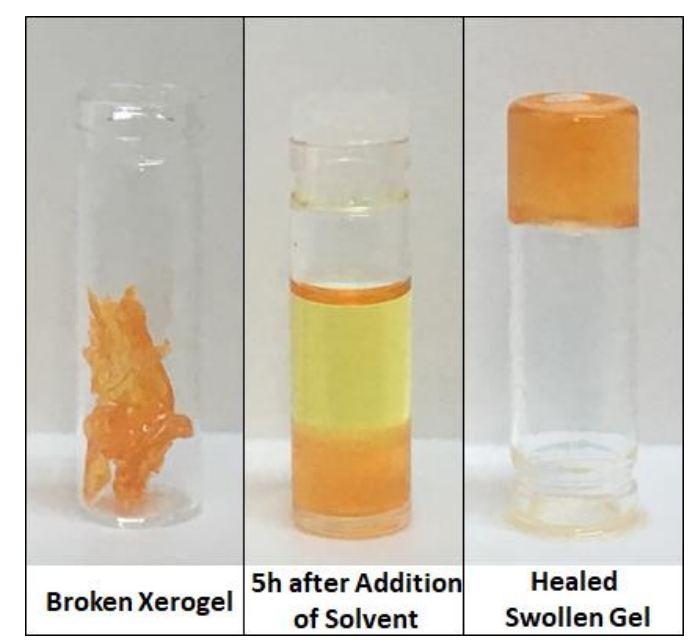

Figure 3.16 Self-healing of poly(FLP) xerogel. 
A poly(FLP) xerogel was broken by a scalpel, and then the broken pieces were put into a small vial. After that, toluene was added into the sample vial in order to promote polymer chain diffusion. The gel pieces started to adsorb solvent and gained some fluidity allowing them to settle down to the bottle of the sample vial. After only about $5 \mathrm{~h}$ of solvent addition, the initial healing of the gel was finished, as shown in the middle figure above. To ensure complete healing, the polymer gel was stood overnight. By this time the gel had full swollen and healed and had recovered most of its strength.

Since the solvent-promoted chain diffusion should be a key factor affecting the healing speed, the self-healing test was also performed with a fully swollen gel, as shown below in Figure 3.17.

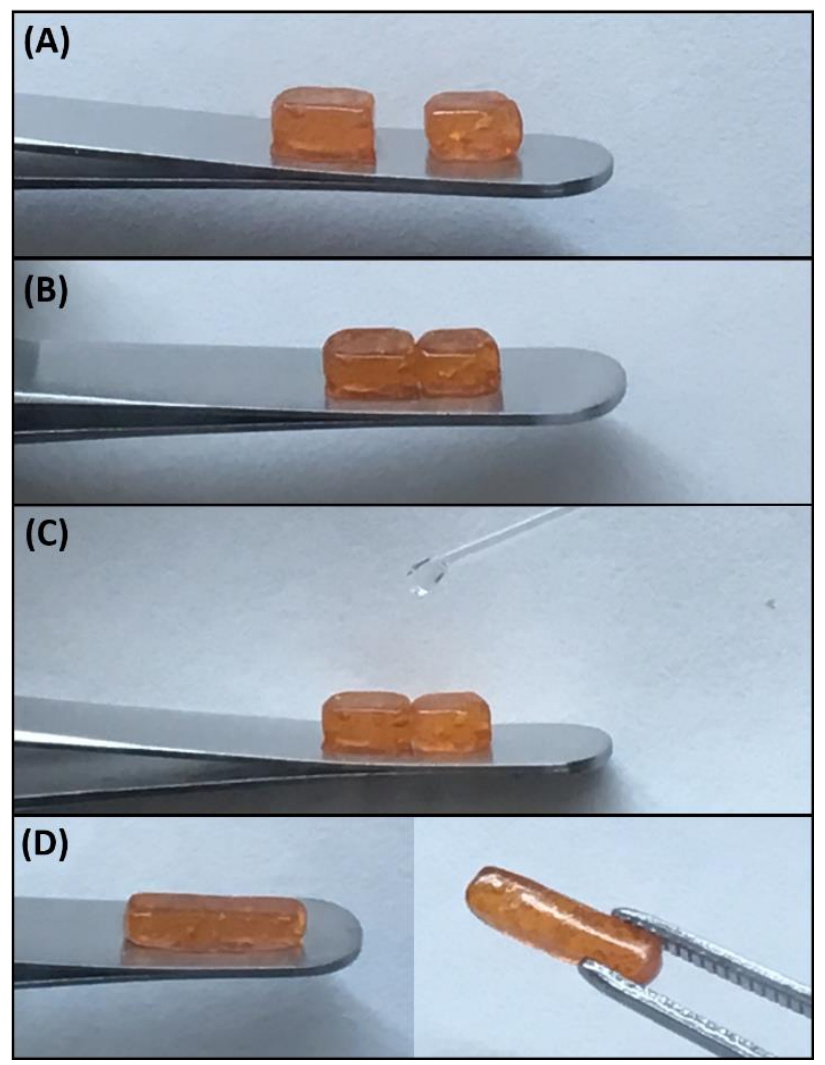

Figure 3.17 Self-healing of fully swollen poly(FLP) gel. 
The fully swollen gel was made into a brick shape and then cut into two pieces (Figure 3.17 (A)). The cut bricks were brought together so that the interfaces closely attached, which allowed inter-chain diffusion to occur between the interfaces (Figure 3.17 (B)). The self-healing can start at this stage. Feeding the interfaces with several drops of solvent will promote even faster healing (Figure 3.17 (C)). In this case, the selfhealing was very fast with only a few minutes being required for complete healing. As shown in Figure 3.17 (D), after healing the cut interfaces were no longer visible, which indicated that healing was achieved at the molecular level. The healing time could be further suppressed to within a minute if the cut bricks were immersed in toluene completely.

The self-healing activity was also investigated by rheology. The sample's self-healing was tested by repeated cycles of cracking and healing. The results are shown below in

Figure 3.18.

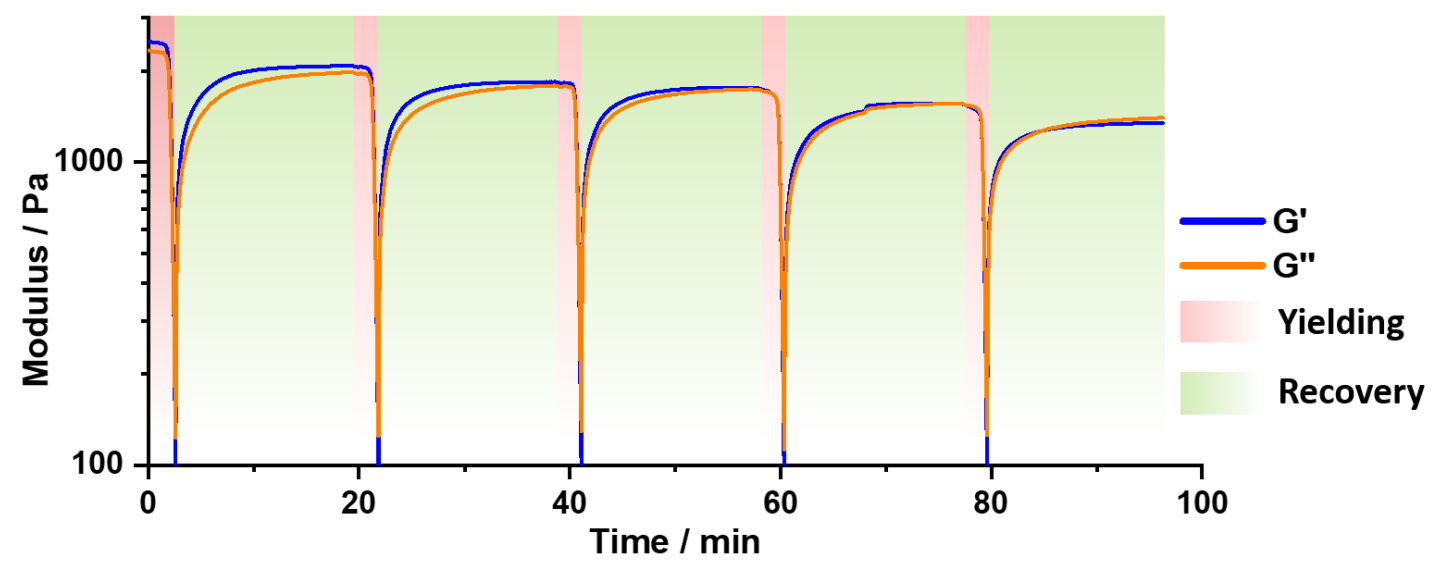

Figure 3.18 Rheology characterisation of self-healing of poly(FLP) gel.

Cracking was achieved by a stress-sweep test with gradually increased stress/strain. When the strain went up to $80 \%$, a dramatic drop in both storage and loss modulus was observed, indicating the rupture of the polymer gel. After that, the strain was set to be $1 \%$ to allow the self-healing to occur. As shown in the figure, both G' and G" recovered quickly during this time. After that, a stress-sweep was again applied to crack the gel. 
This yielding-recovery cycle was repeated five times, and the polymer gel showed a continuous ability to self-heal. The healing efficiency in G' and G" was determined to be $89 \%$ and $91 \%$ respectively. In theory, an intrinsic system should be able to heal an infinite number of times without any decrease in the mechanical properties. The small drop in $G^{\prime}$ and $G^{\prime}$ ' observed in the poly(FLP) system was probably caused by hydrolysis after each cracking, which destroyed the internal structures of the gel.

\subsection{DEAD Triggered Poly(CLP) Gel}

As stated in previous sections, the less sterically hindered monomers, $\mathbf{S t B P h}$ and StPPh2, were able to form a weak conventional Lewis pair adduct, which is not particularly desired for the primary purpose for this project. This binding was very weak and concentration/solvent-dependent, however; and it would therefore be interesting to know if a Lewis pair crosslinked polymeric gel could be obtained from these two monomers. To verify that, the binding between the two monomers with DEAD was first tested by $\mathrm{NMR}$ using a non-coordinating solvent, $\mathrm{CDCl}_{3}$, as shown below in Figure 3.19. The concentration of the mixed monomer solutions was kept low so that there was no or very slow binding between them.

Compared to Figure 3.1, the replacement of PhPMes2 by StPPh2 resulted in much clearer NMR spectra, probably due to the much reduced steric hindrance around the binding centres. Similarly to when $\mathbf{P h P M e s} 2$ was used, StPPh 2 reacted with DEAD to give a mono-adduct ( $\delta 29.3$ in $\left.{ }^{31} \mathrm{P} \mathrm{NMR}\right)$, then bound to boron $\left(\delta 51.9 \mathrm{in}{ }^{31} \mathrm{P} \mathrm{NMR}\right.$ and

$\delta 1.5$ in ${ }^{11} \mathrm{~B}$ NMR). By ${ }^{31} \mathrm{P}$ NMR, the relative integrals of the mono-adduct intermediate StPPh2-DEAD and desired product StPPh2-DEAD-StBPh2 were found to be $86 \%$ and 14\% respectively. The much higher abundance of $\mathbf{S t P P h} \cdot \mathbf{D E A D} \cdot \mathbf{S t B P h} \mathbf{h}_{2}$ indicated a stronger association between the Lewis pair and DEAD and possibly a reduced dynamic nature. There was only one broad peak observed for the desired product in 
${ }^{31} \mathrm{P}$ NMR, which was due to the almost identical three aryl-groups on the phosphorus that reduced the effect of isomerisation. Coordination of StPPh2-DEAD mono-adduct to boron was confirmed by ${ }^{11} \mathrm{~B}$ NMR spectra, with a peak with very low chemical shift being observed for the product, indicating a strong binding by negatively charged nitrogen. Although StPPh2 and StBPh2 can bind to each other, their successful activation of DEAD indicated that they could potentially be used as a masked FLP, which is defined by Lammertsma and Uhl et al. in 2012. ${ }^{13-14}$

(A)
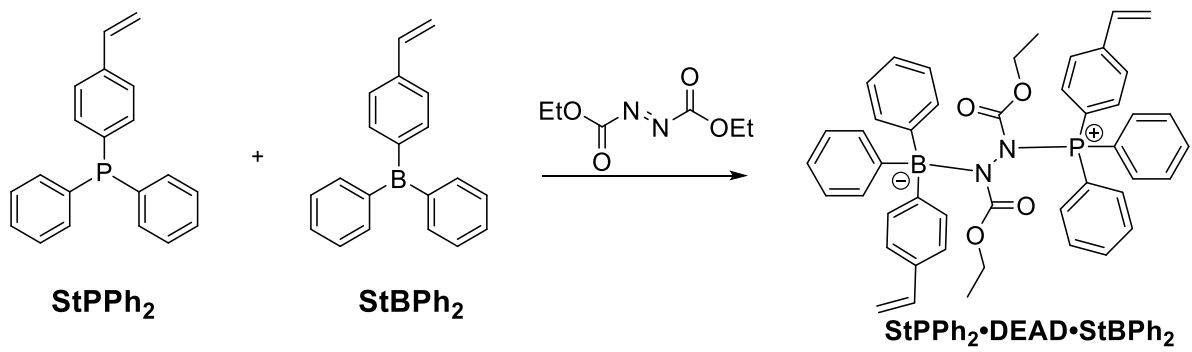

(B)

$$
\text { ตุ }
$$

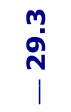

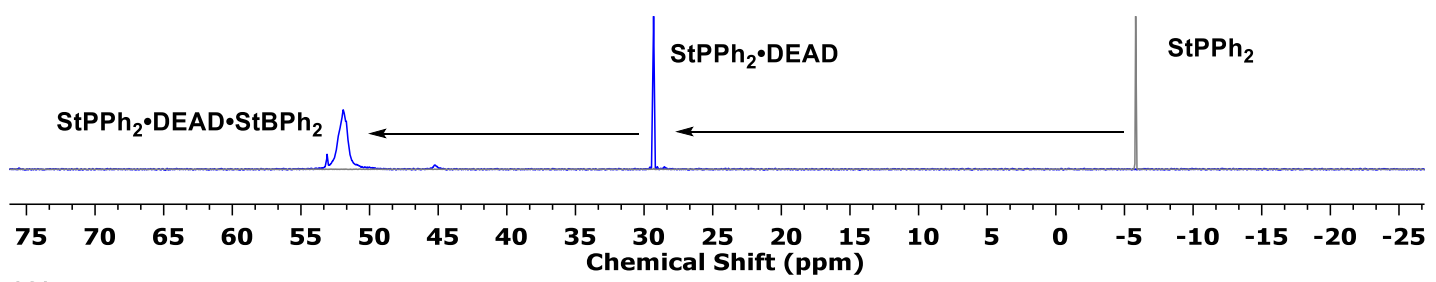

(C)

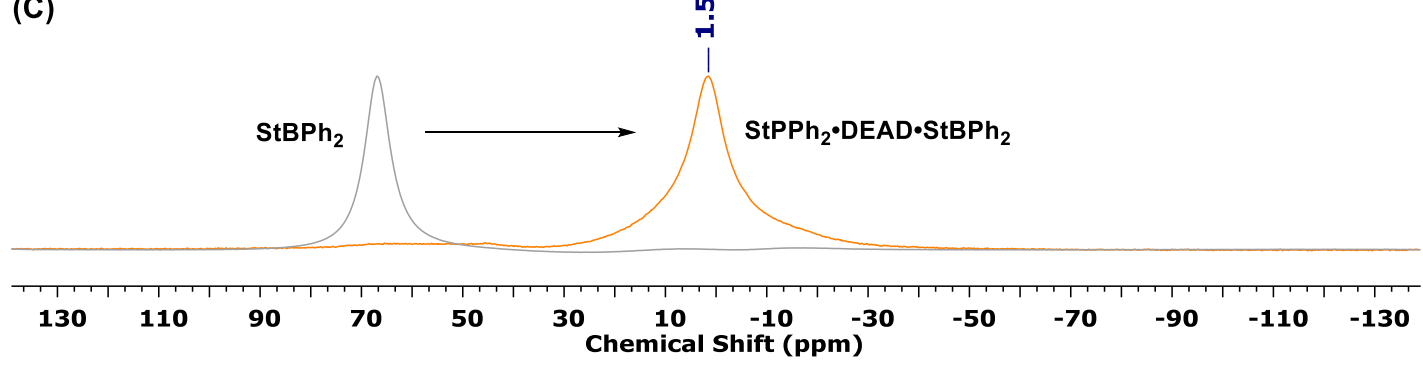

Figure 3.19 (A) Schematic representation of binding experiments using equivalent amounts of StPPh2, StBPh2 and DEAD; overlapped (B) ${ }^{31} \mathrm{P}$ NMR spectra and (C) ${ }^{11} \mathrm{~B}$ NMR spectra of binding reaction mixture (blue and orange colour) and unbound monomers (grey).

After the binding test, the monomers were copolymerised with styrene by RAFT polymerisation using similar reaction conditions to those stated in the previous chapter. 
The resultant copolymers, B1 (7.5 \% boron) and P4 (7.0\% phosphorus), had similar Lewis center content and molecular weight. From the previous NMR binding study, it could be easily predicted that the mixing of $\mathbf{B} 1$ and $\mathbf{P 4}$ should result in a conventional Lewis pair crosslinked polymeric network, which potentially is stimuli-responsive and even self-healable. For this project, however we wished the gelation to be a controlled process that can only be triggered by a small molecule, and any gelation prior to the addition of this small molecule was therefore not preferred. To achieve that, THF was used to dissolve the polymers instead of toluene. According to the binding experiment described in Section 3.1.1, THF should be a stronger donor and thus prevent binding between StPPh2 and StBPh2, and indeed, there was no gelation when the THF solutions of the two polymers were mixed. DEAD was then added into the solution to trigger the gelation, which is shown below in Scheme 3.3.

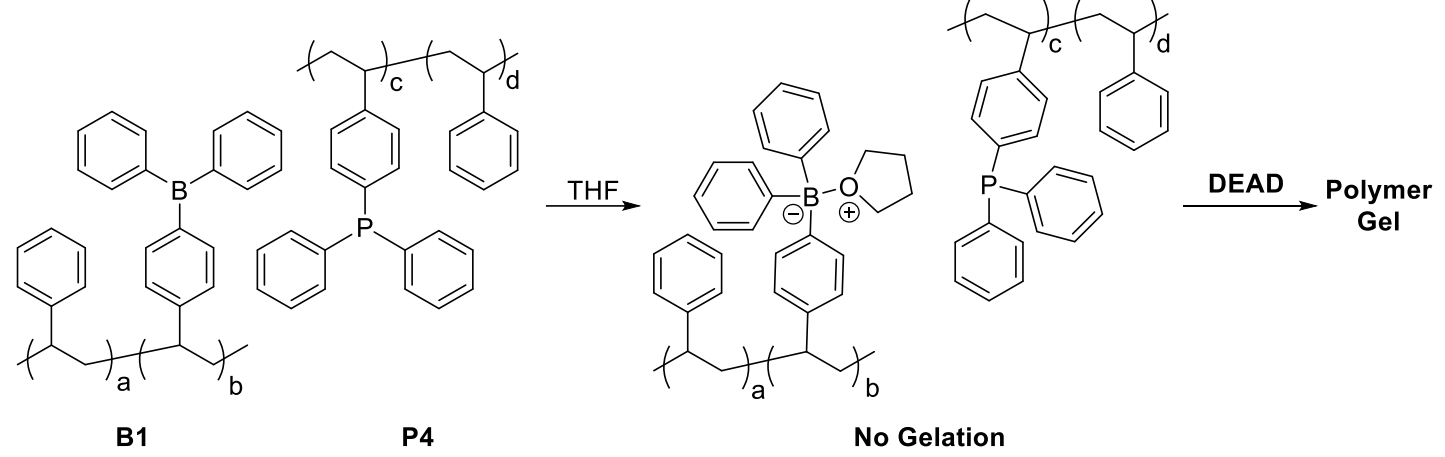

Scheme 3.3 Gelation experiment using P4, B1 and DEAD.

Once DEAD was added, the gelation happened in seconds in a similar manner to that using FLP as crosslinking: first the solution became non-transparent, then it formed a homogeneous gel and crash out of solvent quickly. A reduction in volume was also observed, indicating the dynamic nature of the crosslinking. The gel had a similar physical performance to that the poly(FLP) gel, which is a soft gel with some fluidity when fully swollen. The gel also has some self-healing ability, which was simply verified by cutting and re-joining the solvent-immersed gel. The chemistry behind crosslinking could be either replacement or ring-opening of THF by phosphorus- 
DEAD adduct (Scheme 3.4), although the latter is not very likely to occur due to the relatively mild Lewis acidity of boron. This was not investigated in more detail, however, since, as stated previously, such a system is not strictly within the remit of this project, so no in-depth related research has been attempted yet. Nonetheless, this very early investigation showed that macromolecular conventional Lewis pairs could also successfully generate a small molecule-triggered polymer gel system, as an alternative route to a poly(FLP) system.

(A)

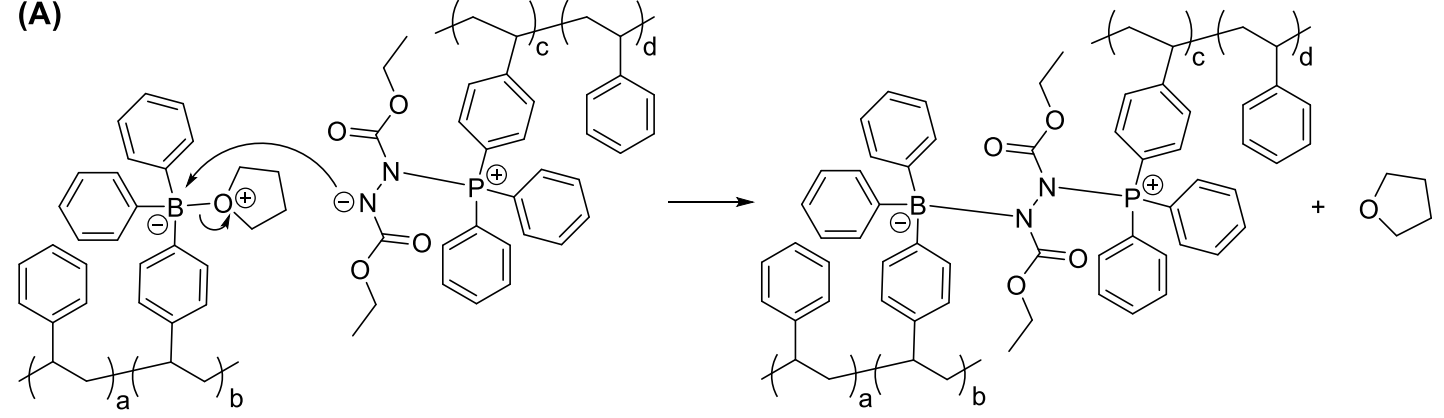

(B)

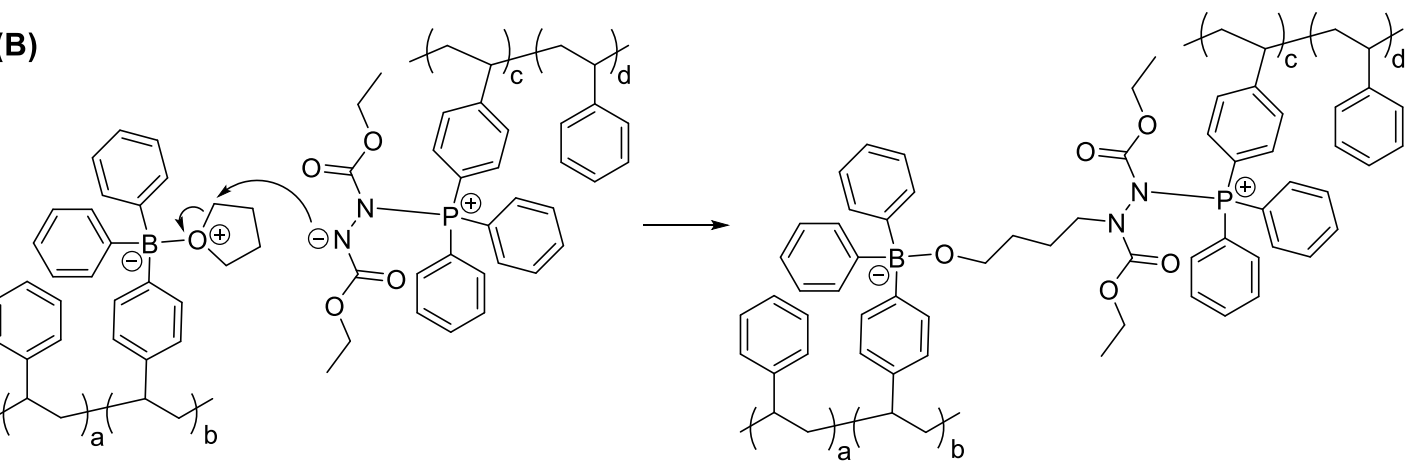

Scheme 3.4 Proposed mechanism for crosslinking via (A) replacement and (B) ringopening of THF by phosphorus-DEAD adduct.

\subsection{Conclusion}

The first example of a polymeric-based frustrated Lewis pair network was successfully synthesised and characterised. The styrene-based copolymers obtained from StPMes2 and StBPh2 were mixed without binding, and the gelation was triggered only when a small molecule, DEAD, was added. The dynamic nature of crosslinking was 
confirmed by NMR study and frequency-sweep rheology. The crosslinking was proved to be exchangeable at room temperature. The poly(FLP) gel was also confirmed to have time-temperature dependence, which is a typical behaviour of a supramolecular polymer network. The temperature responsiveness and self-healing properties of the gel were also confirmed both experimentally and by rheology. As a result, the polymer gel was found to be able to return to a single chain polymer by heating, and ultra-fast self-healing could also be achieved in the presence of a solvent.

Apart from the work presented here, the boron and phosphorous monomers were also copolymerised with methyl methacrylate to study the effect of polymer backbone stiffness on the mechanical properties of a gel, investigated by my colleague, Utku Yolsal. Extensive rheology characterisations were performed, which as a result, poly(FLP-co-MMA) gel also showed similar temperature responsiveness and selfhealing properties, although with some differences in mechanical properties compared to the styrene-based poly(FLP) gel.

The majority of the work described in this chapter, together with that in Chapter Three, has been peer reviewed and accepted for publication. ${ }^{15}$ A paper about the rheology test results described in this chapter has also been submitted for peer review and accepted.

Lastly, this chapter has also briefly described network formation using copolymers obtained from $\mathbf{S t P P h}$ and $\mathbf{S t B P h}$. Although the phosphorus and boron moieties in this case were proved to form a conventional Lewis pair adduct, the binding was very weak and could easily be replaced by a donating solvent, THF. Inspired by this, these two copolymers were mixed after being dissolving in THF. As expected, the THF worked as a protecting group in respect to boron; hence no gelation occurred between the copolymers. The addition of DEAD could still trigger the gelation between polymer chains, however, resulting in the formation of a polymer network, although 
the mechanism by which the crosslinks were formed is as yet unknown. Since this was not a part of this project, however, this system was not further investigated here. Nonetheless, it could still be a good example confirming that is possible to prepare a polymeric gel using a masked frustrated Lewis pair or protected conventional Lewis pairs as crosslinking.

\subsection{References}

[1] Moebs-Sanchez, S.; Bouhadir, G.; Saffon, N.; Maron, L.; Bourissou, D., Chem. Commun. 2008, (29), 3435-3437.

[2] Young, R. J.; Lovell, P. A., Introduction to Polymers. 3rd Edition ed.; CRC Press, Taylor \& Francis Group: the United States, 2011; p 526.

[3] Kavanagh, G. M.; Ross-Murphy, S. B., Prog. Polym. Sci. 1998, 23 (3), 533562.

[4] McCrum, N. G.; P., B. C.; Bucknall, C. B., Principles of Polymer Engineering. Oxford University Press: the United States, 1997; p 128-131.

[5] Adzima, B. J.; Aguirre, H. A.; Kloxin, C. J.; Scott, T. F.; Bowman, C. N., Macromolecules 2008, 41 (23), 9112-9117.

[6] Röttger, M.; Domenech, T.; van der Weegen, R.; Breuillac, A.; Nicolaÿ, R.; Leibler, L., Science 2017, 356 (6333), 62-65.

[7] Roberts, M. C.; Hanson, M. C.; Massey, A. P.; Karren, E. A.; Kiser, P. F., Adv. Mater. 2007, 19 (18), 2503-2507.

[8] Kloxin, C. J.; Scott, T. F.; Adzima, B. J.; Bowman, C. N., Macromolecules 2010, $43(6), 2643-2653$

[9] McCrum, N. G.; P., B. C.; Bucknall, C. B., Principles of Polymer Engineering. Oxford University Press: the United States, 1997; p 150-153.

[10] Young, R. J.; Lovell, P. A., Introduction to Polymers. 3rd Edition ed.; CRC 
Press, Taylor \& Francis Group: the United States, 2011; p 503-505.

[11] Noro, A.; Hayashi, M.; Matsushita, Y., Soft Matter 2012, 8 (24), 6416-6429.

[12] Noro, A.; Matsushita, Y.; Lodge, T. P., Macromolecules 2008, 41 (15), 58395844.

[13] Appelt, C.; Westenberg, H.; Bertini, F.; Ehlers, A. W.; Slootweg, J. C.; Lammertsma, K.; Uhl, W., Angew. Chem., Int. Ed. 2011, 50 (17), 3925-3928.

[14] Roters, S.; Appelt, C.; Westenberg, H.; Hepp, A.; Slootweg, J. C.; Lammertsma, K.; Uhl, W., Dalton Trans. 2012, 41 (30), 9033-9045.

[15] Wang, M.; Nudelman, F.; Matthes, R. R.; Shaver, M. P., J. Am. Chem. Soc. 2017, 139 (40), 14232-14236. 


\section{Chapter Four. Novel Boron-Containing Monomers for Next Generation Polymeric Frustrated Lewis Pairs}

As stated in previous chapters, a polymer network was successfully generated from the monomers StBPh2 and StPMes2. The network is temperature-responsive and selfhealable due to the dynamic nature of the crosslinking. These properties were also confirmed by rheology studies. Although some success has been achieved, a limitation of this system is that the scope of the small molecule that can be activated is severely limited to azo-class compounds due to the intermediate Lewis acidity of boron functionalities. A more Lewis acidic boron monomer therefore needs to be designed and synthesised to improve the overall reactivity of the FLP. The most direct method is to introduce fluorinated or trifluoromethyl-substituted phenyl rings onto the boron, which will improve the electron withdrawing ability of the whole molecule. While we were exploring the methods to synthesise these new boron compounds, Yan et al. reported their work about $2^{\text {nd }}$ generation poly(FLP). ${ }^{1}$ The synthetic method used for boron monomer was based on our published coordination-recovery strategy, which is shown below in Scheme 4.1.

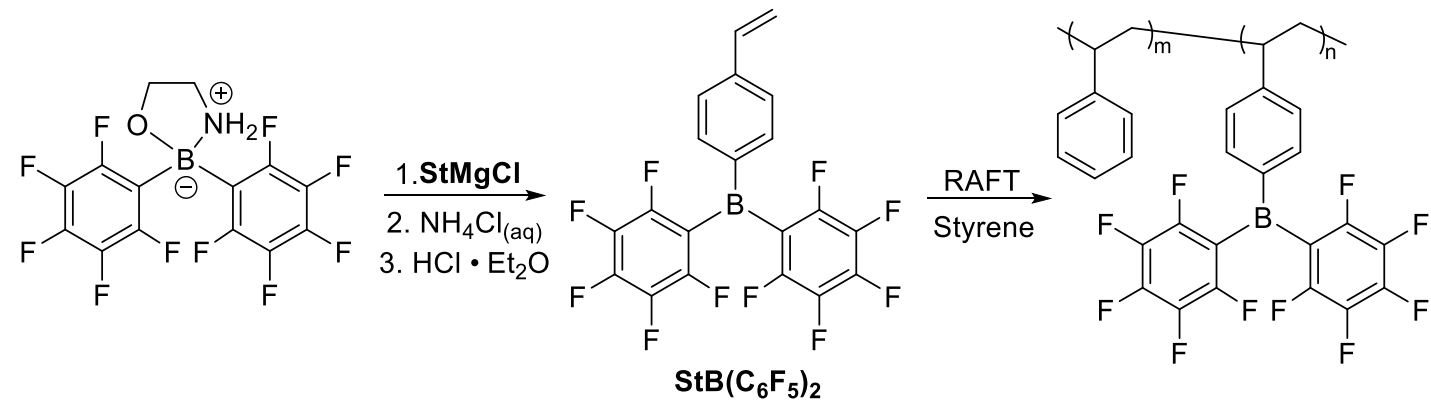

Scheme 4.1 Fluorinated-aryl substituted borane-containing polymers reported by Yan et al. ${ }^{1}$ 
The poly(FLP) was generated using the same phosphorus-containing copolymer. Because of the enhanced Lewis acidity of boron, the system was able to capture $\mathrm{CO}_{2}$ as crosslinker reversibly. Furthermore, due to the di-block structure of the copolymers, the resultant polymer gel was micellar, with the micelles having an average diameter of $40 \mathrm{~nm}$. The micelles were also proven to be able to catalyse an amine formylation reaction, and the polymers can be easily recycled after use. After this work, they also reported other polymer gels generated from small molecule-based FLPs, where gelation was triggered in a similar way by capturing $\mathrm{CO}_{2} \cdot{ }^{2-3}$

Although Yan et al. have reported several examples of fluorinated-aryl-substituted borane-containing poly(FLP), their work focused purely on the goal of carbon capture, and no dihydrogen cleavage was reported using these systems, probably due to the Lewis acidity being reduced by the presence of one non-fluorinated phenyl substituent on the boron. Also the introduction of donating functional groups such as nitrile and sulphur due to the incorporation of AIBN and RAFT agent in polymerisations may interrupt the boron moieties in the system. We therefore decided to explore a novel poly(FLP) system with an even more Lewis acidic boron but free of undesired donating functionalities. The goal of this macromolecular system includes activation of multiple small molecules $\left(\mathrm{CO}_{2}\right.$ and $\left.\mathrm{H}_{2}\right)$ at the same time, hence achieving one-pot carbon reduction. Although this remains incomplete work, the synthesis of some new boron and phosphorus monomers and some initial studies exploring their use as catalysts are discussed in the following sections. 


\section{1 (2,3,5,6-tetrafluorostyryl) -bis- (pentafluorophenyl)borane $\left(\mathrm{StF}_{4}\right) \mathrm{B}\left(\mathrm{C}_{6} \mathrm{~F}_{5}\right)_{2}$}

\subsubsection{Synthesis of $\left(\mathrm{StF}_{4}\right) \mathrm{B}\left(\mathrm{C}_{6} \mathrm{~F}_{5}\right)_{2}$}

As stated in previous sections, we decided to make a more Lewis acidic boroncontaining monomer, in which all three aryl substituents are fluorinated. The synthesis of this borane monomer was performed using a similar method to that used for tris(pentafluorophenyl)borane (BCF). Since the desired monomer product is not fully symmetrical compared to BCF, two different Grignard reagents needed to be added to boron trifluoride in sequence. This one-pot two-step reaction is set out in Scheme 4.2 below.<smiles>CCOC(=O)c1c(F)c(F)c(F)c(F)c1Br</smiles><smiles>[15NH]c1c(F)c(F)c(F)c(F)c1F</smiles>
$\left(\mathrm{C}_{6} \mathrm{~F}_{5}\right) \mathrm{MgBr}$

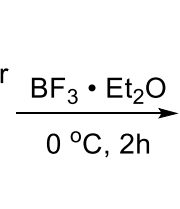
${ }^{\circ} \mathrm{C}, 2 \mathrm{~h}$<smiles>FB(c1c(F)c(F)c(F)c(F)c1F)c1c(F)c(F)c(F)c(F)c1F</smiles>
$\left(\mathrm{C}_{6} \mathrm{~F}_{5}\right)_{2} \mathrm{BF}$

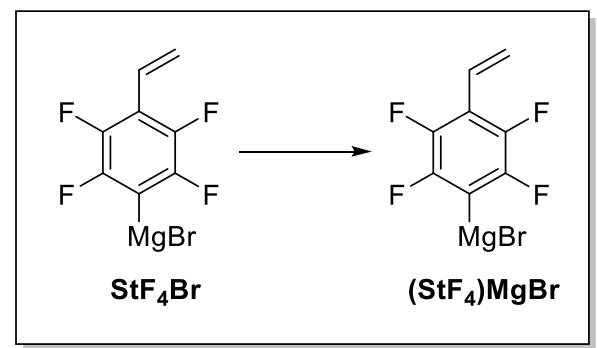<smiles>C=Cc1c(F)c(F)c(B(c2c(F)c(F)c(F)c(F)c2F)c2c(F)c(F)c(F)c(F)c2F)c(F)c1F</smiles>

Scheme 4.2 Synthetic route for fluorinated-aryl substituted borane $\left(\mathrm{StF}_{4}\right) \mathbf{B}_{(}\left(\mathbf{C}_{6} \mathbf{F}_{5}\right)_{2}$.

Bis(pentafluorophenyl)borane fluoride $\left(\mathbf{C}_{6} \mathbf{F}_{5}\right)_{2} \mathbf{B F}$ was first made in situ, and then reacted with the Grignard reagent $\left(\mathbf{S t F}_{4}\right) \mathbf{M g B r}$. This synthetic method should be straightforward since both the Grignard $\left(\mathbf{C}_{6} \mathbf{F}_{5}\right) \mathbf{M g B r}$ and the intermediate $\left(\mathbf{C}_{6} \mathbf{F}_{5}\right)_{2} \mathbf{B F}$ 
can be produced efficiently with the generation of very few side-products. As stated previously in Chapter Two, the use of THF should be avoided for this synthesis since it can bind to the boron product, which makes purification very difficult. Hence diethyl ether was used as the solvent throughout the whole reaction. The Grignard reagent, (StF4)MgBr, was generated from a novel compound, 4-bromo-2,3,5,6tetrafluorosytrene, $\mathrm{StF}_{4} \mathbf{B r}$. Although it has been proven that the $\mathrm{Et}_{2} \mathrm{O}$ cannot promote the formation of Grignard for either 4-chlorostyrene or 4-bromostyrene, it was predicted to work for $\mathrm{StF}_{4} \mathrm{Br}$ due to the stabilisation by the lone pairs from the orthofluorine atoms. The first step in the reaction was the preparation of $\mathbf{S t F}_{4} \mathbf{B r}$, as shown below in Scheme 4.3.

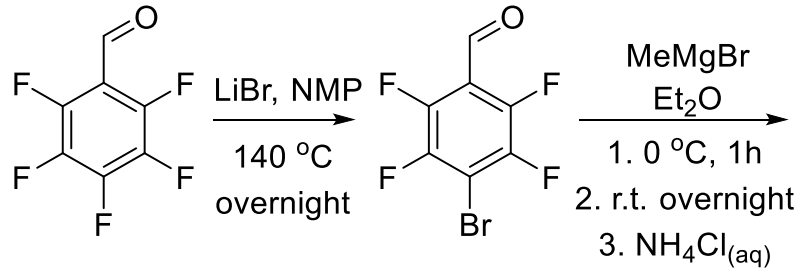

$83.5 \%$<smiles>CC(O)c1c(F)c(F)c(Br)c(F)c1F</smiles>

$68.7 \%$

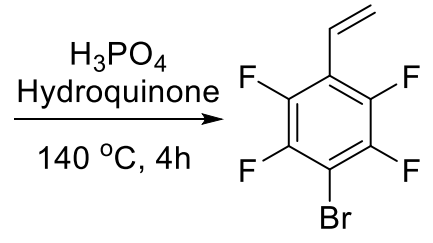

$\mathrm{StF}_{4} \mathrm{Br}$ $58.9 \%$

Scheme 4.3 Synthesis of $\mathrm{StF}_{4} \mathrm{Br}$.

The reaction started from a halogen exchange reaction of a commercially available compound, pentafluorobenzaldehyde. Treatment of this compound with lithium bromide at $140{ }^{\circ} \mathrm{C}$ resulted in the replacement of the para-fluorine atom into the bromine group. Then, the newly synthesised benzaldehyde was reacted with methylmagnesium bromide Grignard reagent to generate secondary alcohol. The last step was the dehydration reaction performed using phosphoric acid at $140{ }^{\circ} \mathrm{C}$ for $4 \mathrm{~h}$. Hydroquinone was put into the reaction mixture before dehydration in order to minimise any self-polymerisations. Dehydration can also be achieved at low temperature by using a special catalyst, copper sulphate embedded silica gel. The $\mathrm{StF}_{4} \mathrm{Br}$ product can be readily purified by flushing through a silica plug using nonpolar hexane, on account of its very different polarity compared to the starting 
materials. The whole synthetic procedure was robust, with high conversion in each step, which means purification after each step was not very necessary.

After $\mathbf{S t F}_{\mathbf{4}} \mathbf{B r}$ had been synthesised, attention turned to investigating its use in Grignard preparation. As expected, unlike its un-fluorinated analogue, $\mathrm{StF}_{4} \mathbf{B r}$ can successfully generate Grignard reagents with bulk magnesium in $\mathrm{Et}_{2} \mathrm{O}$. Upon addition of $\mathbf{S t F} \mathbf{B} \mathbf{B r}$, the ether solution quickly changed into a deep brown colour. A dark coloured floccule was also generated in the solution, however, which was thought to be the self-coupled by-product of the Grignard. To minimise this side reaction, the reaction was controlled at $0{ }^{\circ} \mathrm{C}$, and $\mathrm{StF}_{4} \mathbf{B r}$ was diluted in ether $(0.3 \mathrm{M})$ before being added into the mixture of magnesium and diethyl ether. The addition was controlled to be very slow with a speed of $0.6 \mathrm{~mL} / \mathrm{min}$. The resultant Grignard reagent was titrated to monitor the conversion of reaction, which was only $56.4 \%$ on average. Other methods to prepare Grignard were also attempted such as Grignard exchange or a $\mathrm{LiCl}$ mediated reaction. No noticeable improvement was observed, however, and a large amount of visible byproduct was seen in all cases. Hence it was decided to use an excess amount of $\mathrm{StF}_{4} \mathbf{B r}$ for synthesising the boron monomer, since both the self-coupled by-product and unreacted $\mathbf{S t F}_{4} \mathbf{B r}$ during Grignard preparation should be able to be removed during purification.

With all reagents in hand, the synthesis of boron monomer $\left(\mathbf{S t F}_{4}\right) \mathbf{B}_{\left(\mathbf{C}_{6} \mathbf{F}_{5}\right)_{2} \text { was }}$ performed. First, a slightly excess amount of Grignard reagent $\left(\mathbf{C}_{6} \mathbf{F}_{5}\right) \mathbf{M g B r}$ was prepared from bromopentafluorobenzene in $\mathrm{Et}_{2} \mathrm{O}$, then added dropwise into the boron trifluoride etherate solution to produce the intermediate $\left(\mathbf{C}_{6} \mathbf{F}_{5}\right) \mathbf{B F}$. Then, the prepared Grignard solution $\left(\mathbf{S t F}_{4}\right) \mathbf{M g B r}$ was added dropwise into the reaction mixture at $0{ }^{\circ} \mathrm{C}$. The mixture was stirred overnight at room temperature. After the reaction, different work-up methods were attempted. Initially, a similar work-up procedure to that used for the synthesis of BCF was attempted, wherein the solvent of the reaction mixture 
was first replaced by toluene in order to remove any $\mathrm{Et}_{2} \mathrm{O}$ that was bound to the product. This was achieved by introducing a bulk amount of toluene and heating to around $100{ }^{\circ} \mathrm{C}$ under a vacuum. This method led to a dramatic decrease in yield, however, probably due to the self-polymerisation of product, which led to very difficult recrystallisation. Hence, instead, the $\mathrm{Et}_{2} \mathrm{O}$ in the reaction was simply vacuumed off at room temperature for a long period of time, and then hexane was introduced to extract the product. A quantitative yield was achieved by this method, which was $46.6 \%$ after repeated recrystallisation from hexane. The obtained product $\left(\mathbf{S t F}_{4}\right) \mathbf{B}_{(}\left(\mathbf{C}_{6} \mathbf{F}_{5}\right)_{2}$ was characterised by NMR spectroscopy, as shown below in Figure 4.1.

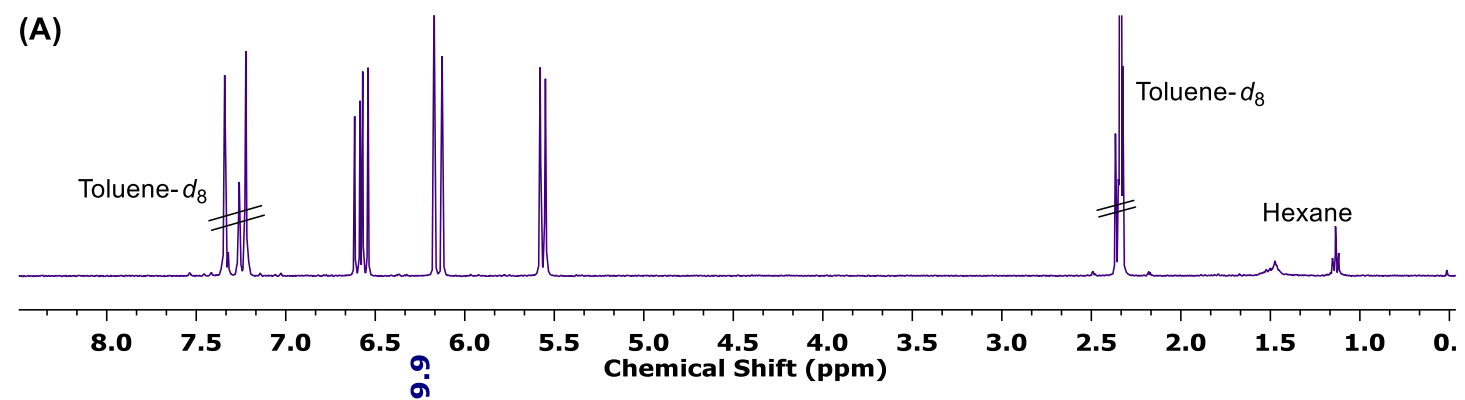

(B)

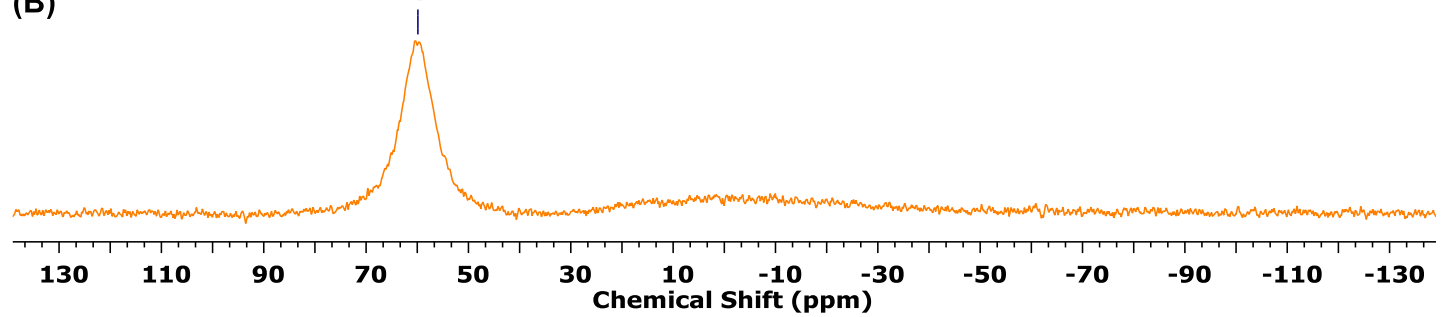

(C)

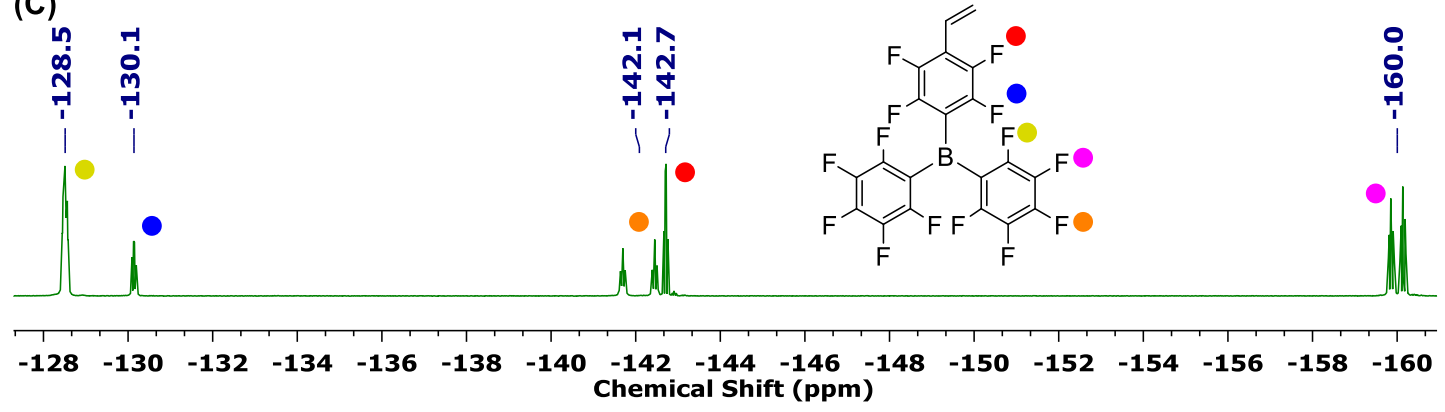

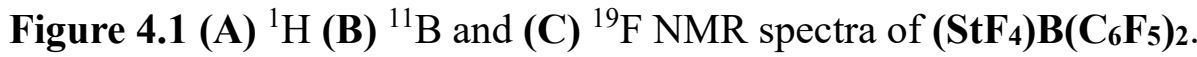

The ${ }^{1} \mathrm{H}$ NMR spectrum shows only three peaks, corresponding to the vinyl protons of the product. The product gave a broad peak at $59.9 \mathrm{ppm}$ in the ${ }^{11} \mathrm{~B}$ NMR spectrum, 
which is very similar to its analogue BCF $(59.8 \mathrm{ppm}) .{ }^{4}$ It is worth noting that the monomer $\mathbf{S t B}\left(\mathbf{C}_{6} \mathbf{F}_{5}\right)_{2}$ reported by Yan et al. showed a very different chemical shift of boron at $74.4 \mathrm{ppm},{ }^{1}$ indicating that it may have had an effect on the reactivity of the boron compound by replacing only one of the fluorinated phenyl rings with a nonfluorinated one. The ${ }^{19} \mathrm{~F}$ NMR spectrum, meanwhile, gave five peaks. The fluorine atoms on the pentafluorophenyl groups showed peaks with a very similar chemical shift compared to BCF. The meta-fluorine atoms on the tetrafluorostyryl group were much more deshielded, however, which are very similar to the reported boron compound $\mathrm{B}\left(\mathrm{C}_{6} \mathrm{~F}_{4} \mathrm{H}\right)_{3}$.

The Lewis acidity of the synthesised monomer $\left(\mathbf{S t F}_{4}\right) \mathbf{B}_{(}\left(\mathbf{C}_{6} \mathbf{F}_{5}\right)_{2}$ was tested using the Gutmann-Beckett method, as shown below in Figure 4.2. One equivalent of triethylphosphine oxide was mixed with $\left(\mathbf{S t F}_{4}\right) \mathbf{B}\left(\mathbf{C}_{6} \mathbf{F}_{5}\right)_{2}$ in toluene- $d_{8}$, and the mixture was characterised by ${ }^{31} \mathrm{P}$ NMR spectroscopy. As a result, the $\mathbf{A N}$ of $\left(\mathbf{S t F}_{4}\right) \mathbf{B}\left(\mathbf{C}_{6} \mathbf{F}_{5}\right)_{2}$ was determined to be 75.1 , which is very similar to its analogue, $\mathrm{BCF}$ ( $\mathbf{A N}=76.7$ in toluene- $d 8){ }^{5-6}$

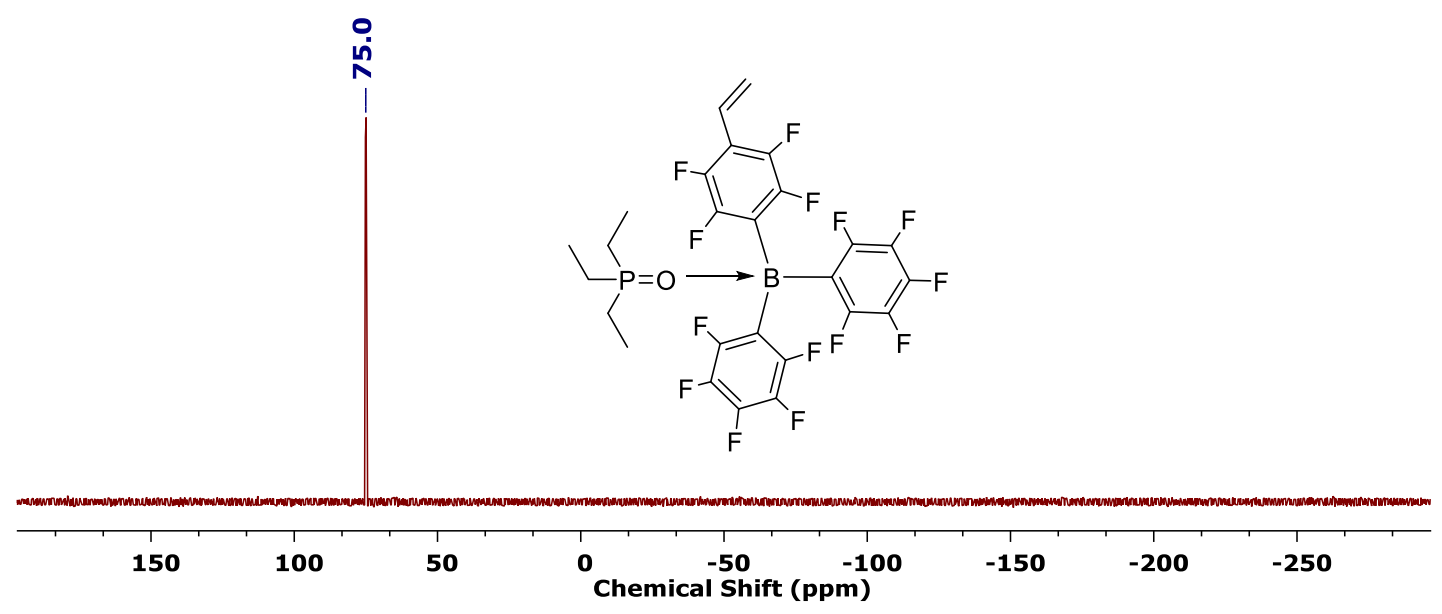

Figure 4.2 ${ }^{31} \mathrm{P}$ NMR spectrum to determine the Lewis acidity of $\left(\mathbf{S t F}_{4}\right) \mathbf{B}\left(\mathbf{C}_{6} \mathbf{F}_{5}\right)$ 2. 


\subsubsection{Binding test with StPMes2}

The synthesised monomer $\left(\mathbf{S t F}_{4}\right) \mathbf{B}\left(\mathbf{C}_{6} \mathbf{F}_{5}\right)_{2}$ was mixed with the phosphine monomer StPMes2 with 1:1 equivalent in toluene- $d_{8}$. Upon mixing the solutions of the boron and phosphorus-containing monomers, the colour of the solution turned from colourless to deep orange, indicating the interactions between the Lewis basic and acidic centres, as shown in Figure 4.3. The resultant mixture was also found to become fluorescent, emitting a weak violet light when irradiated by long-range wavelength ultra-violet lights.

(A)

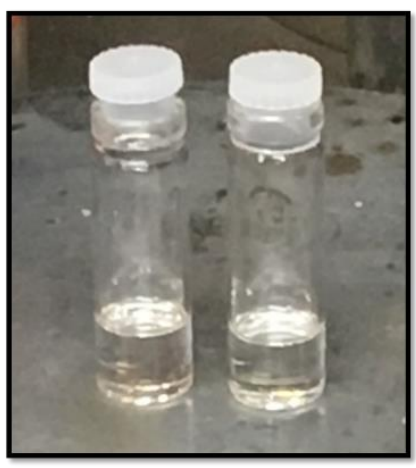

(B)

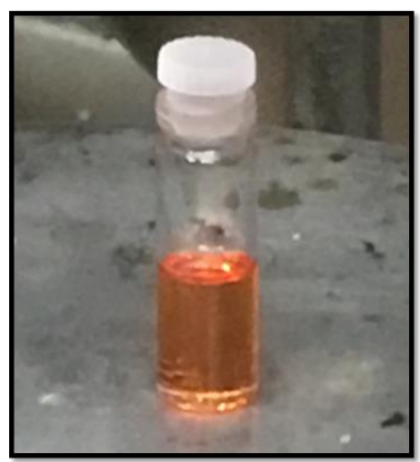

Figure 4.3 Solutions of boron and phosphorus-containing monomers (A) before

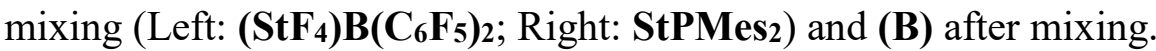

The ${ }^{1} \mathrm{H},{ }^{11} \mathrm{~B}$ and ${ }^{31} \mathrm{P}$ NMR spectroscopy were performed to characterise the mixed solution. The ${ }^{1} \mathrm{H}$ NMR spectroscopy showed two sets of vinyl peaks, indicating that both the styryl groups in the boron and phosphorus-containing monomers were tolerant to the Lewis pairs. No changes were observed from the ${ }^{11} \mathrm{~B}$ and ${ }^{31} \mathrm{P}$ NMR spectra in the chemical shifts of the boron and phosphorus peaks, which confirmed FLP formation between the two monomers $\left(\mathbf{S t F}_{4}\right) \mathbf{B}\left(\mathbf{C}_{6} \mathbf{F}_{5}\right)_{2}$ and $\mathbf{S t P M e s} 2$. The colour change mentioned earlier was therefore not due to the formation of a CLP adduct. Colour changes have been observed in other FLP systems, and have been explained as possibly resulting from the $\pi$-stacking between the two aromatic compounds caused by the pre-orientation between them (here due to the interactions between the boron and phosphorus). 


\subsubsection{Polymerisations}

We used various methods in an attempt to copolymerise the obtained highly Lewis

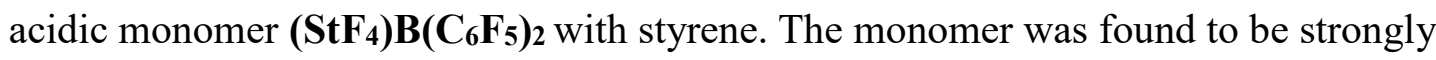
exothermic when mixed with styrene in bulk, and polymerisation was initiated instantly. In all cases, therefore, the two monomers were diluted and mixed dropwise. RAFT polymerisation was attempted initially and NMR spectroscopy revealed that the boron monomer was able to bind with all polymerisation reagents that contain donating functionalities. The ${ }^{11} \mathrm{~B}$ NMR spectrum of the mixture of $\left(\mathbf{S t F}_{4}\right) \mathbf{B}_{(}\left(\mathbf{C}_{6} \mathbf{F}_{5}\right)_{2}$ and the RAFT agent CDB showed two broad peaks at $27.6 \mathrm{ppm}$ and $18.3 \mathrm{ppm}$ respectively, corresponding to the relatively weak bindings of the boron to the two sulphur atoms in the thioester groups. $\left(\mathbf{S t F}_{4}\right) \mathbf{B}\left(\mathbf{C}_{6} \mathbf{F}_{5}\right)_{2}$ was also found to bind weakly with the sterically hindered internal stand TIPT, which was used before for StBPh2. An extremely broad peak at $43.0 \mathrm{ppm}$ with a half-height-width of $26.0 \mathrm{ppm}$ was observed in the ${ }^{11} \mathrm{~B}$ NMR spectrum, indicating the very weak nature of the binding. Another internal standard, 1,2,4,5-tetramethylbenzene, was therefore used for the polymerisations instead. The polymerisation was initially performed at $100{ }^{\circ} \mathrm{C}$ via self-initiation. Some sidereactions occurred, however, and a gel was obtained after the reaction. It was proposed that the side reactions might be minimised by reducing the reaction temperature, hence AIBN was introduced to initiate the RAFT polymerisation at $60{ }^{\circ} \mathrm{C}$. In this case, no gel was generated, although there was no conversion for the polymerisation either. It was found that the binding between $\mathrm{CDB}$ and boron monomers discouraged the cleavage of the C-S bonding and prohibited the chain-transfer. For this reason, RAFT polymerisation was given up, and conventional free radical polymerisations were attempted using AIBN as the initiator. AIBN contains nitrile groups and is able to bind with the boron monomer. Due to its relatively small amount compared to the boron monomer in the polymerisation, however, it was proposed that most of the boron 
moieties should still remain intact after polymerisations. Finally, a soluble branched copolymer of $\left(\mathbf{S t F}_{4}\right) \mathbf{B}\left(\mathbf{C}_{6} \mathbf{F}_{5}\right)_{2}$ and styrene was synthesised, although with a significant loss in yield.

\subsubsection{Gelation Experiment}

A copolymer of styrene and StPMes2 was synthesised by free radical polymerisation using AIBN as the initiator. The resultant phosphorus-containing copolymer had a similar molecular weight to the copolymer synthesised from $\left(\mathbf{S t F}_{4}\right) \mathbf{B}\left(\mathbf{C}_{6} \mathbf{F}_{5}\right)_{2}$. After that, both of the copolymers were dissolved in toluene and mixed together, as shown below in Figure 4.4.
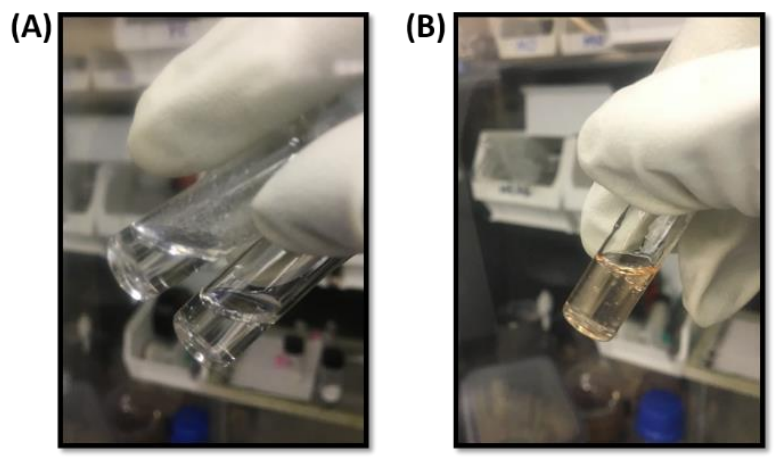

Figure 4.4 Gelation experiment of 2nd generation poly(FLP). (A) Solution of

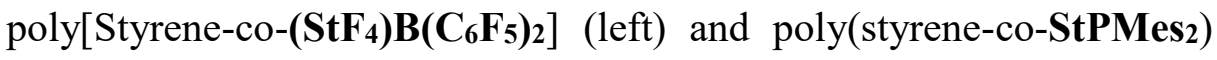
(right). (B) The mixture of the two copolymers.

Similar to the monomer binding test, the colour of the solution turned to pale orange when the two copolymers were mixed, indicating the weak interactions between the boron and phosphorus. The resultant solution had some fluidity from the beginning and became stickier over time. Surprisingly, by leaving the solution at room temperature for about $30 \mathrm{~min}$, a gel-like material was obtained finally. To verify whether a crosslinked material was made, a significant excess amount of solvent was added into this product with vigorous stirring; however, it did not dissolve even after 
the gel was shredded into smaller pieces, and the boundaries between the gel and the solvent were always clear to see.

To make sure the material was not produced by the possible crosslinking between the chain-end nitrile groups (from AIBN) and boron moieties, a control experiment was performed. A homopolymer of styrene was synthesised via free radical polymerisation using AIBN as the initiator. The resultant polystyrene was then mixed with the boroncontaining copolymer. In this case, there was no change in colour observed upon mixing due to the lack of phosphorus moieties. The resultant mixture was very sticky, but it could hold itself intact as a gel upon inversion of its container. When more solvent was added, its viscosity decreased dramatically until it was similar to any other chemical solution, and no solid-liquid interface could be observed. Through this control experiment, it was concluded that the gelation between boron- and phosphoruscontaining copolymers was governed by the weak interactions between the boron and phosphorus moieties. The chain-end nitrile groups might contribute some viscosity to the resultant gel, but were not in charge of gelation. This phenomenon of gelation before triggering by small molecules was not reported by Yan et al., indicating the higher FLP reactivity of our system.

If the crosslinking did indeed come from the weak interactions between the boron and phosphorus centres, the organogel should be temperature responsive. This was tested by a heating-cooling experiment, shown below in Figure 4.5. Upon heating, due to the cleavage of supramolecular crosslinking, the gel visibly gained more fluidity until it had completely turned to a solution. Then, the resultant polymer solution was cooled down in an ice-bath. Within one minute, the gel was reformed by reorganisation of the weak interactions between boron and phosphorus. This healing-cooling cycle was repeated ten times, and no noticeable loss in gel strength was observed. This interesting experimental result indicated that the gel was a smart material that was both heat- 
responsive and self-healing.

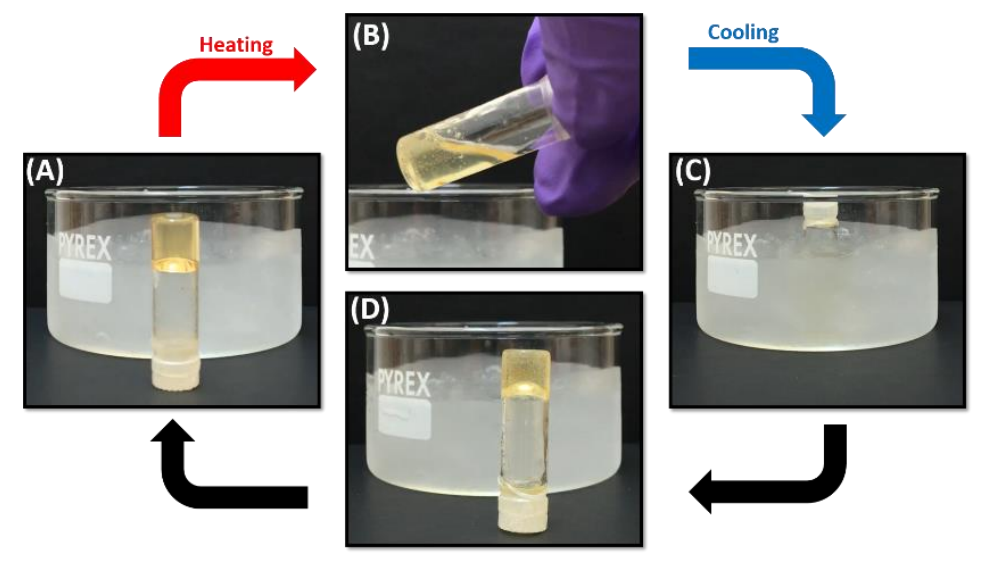

Figure 4.5 Thermal responsiveness of the 2nd gen. poly(FLP) gel. (A) Before heating;

(B) turning to polymer solution after heating; (C) cooling down by ice-bath;

(D) recovered gel.

The promising results discussed above motivated us to improve the system by complete removal of any unwanted donating functionalities from the polymers. Since it seemed difficult to polymerise the highly Lewis acidic boron monomer, a postpolymerisation modification was attempted, as shown below in Scheme 4.4.<smiles>C=Cc1c(F)c(F)c(Br)c(F)c1F</smiles>

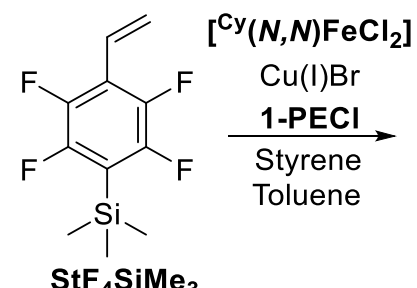

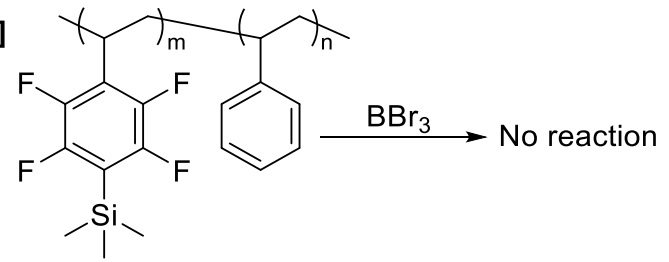
$\mathrm{StF}_{4} \mathrm{SiMe}_{3}$<smiles>CC(Cl)c1ccc([N+](Cl)(Cl)Cl)cc1</smiles>

Scheme 4.4 Attempted post-polymerisation modification reaction to synthesise poly[Styrene-co-(StF 4$\left.) B\left(\mathbf{C}_{6} \mathbf{F}_{5}\right)_{2}\right]$.

Firstly, a silicon-containing fluorinated-styrene monomer, $\mathbf{S t F}_{4} \mathbf{S i M e}$, was synthesised 
from $\mathrm{StF}_{4} \mathrm{Br}$. After that, the silicated monomer was copolymerised with styrene using iron-mediated ATRP polymerisation. ${ }^{7-8}$ A well-controlled polymer was obtained with a chain-end functionality of alkyl chloride, which did not affect the Lewis acidic boron. A boron-silicon exchange reaction was then applied to the copolymer using boron tribromide. After the reaction, however, no exchanged product was observed by NMR spectroscopy. The ${ }^{29} \mathrm{Si}$ NMR spectrum showed that the silicon groups had not been successfully removed, and no boron product could be observed in the ${ }^{11} \mathrm{~B}$ NMR spectrum. This result was in line with that reported in another study, where the author explained that it resulted from the electron withdrawing aryl substituents having too low nucleophilicity, thus prohibiting the boron-silicon exchange. ${ }^{9}$ Other possible exchange reactions include using organotin or organomercury compounds, but this work is still ongoing in our lab.

\subsubsection{Small Molecule Activation}

The FLP reactivity of the new system was probed by using the monomers

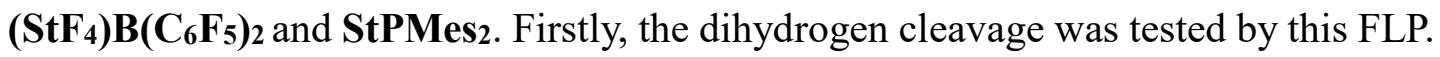
The boron and phosphorus-containing monomers were dissolved in toluene, and the solution was degassed by freeze-pump-thawing three times. After that, one atmosphere of hydrogen gas was introduced into the reaction vessel. Although there was no observation from the beginning of the reaction, the solution changed from a deep orange to a pale yellow colour after stirring overnight. It also became heterogeneous after the reaction, transforming to a sticky yellow oil with a clear solvent on top (Figure 4.6 (A)). It was proposed that the product might have low solubility in toluene, therefore bromobenzene was used instead. This time, after introducing hydrogen gas, the colour of the solution changed to pale yellow after only 30 min (Figure 4.6 (B)). For comparison, dihydrogen activation of $\mathrm{BCF} / \mathrm{StPMes} 2$ was also performed in bromobenzene (Figure 4.6 (C)). In this case, the change in colour from deep orange 
to almost colourless was observed immediately after introducing hydrogen gas.

(A)
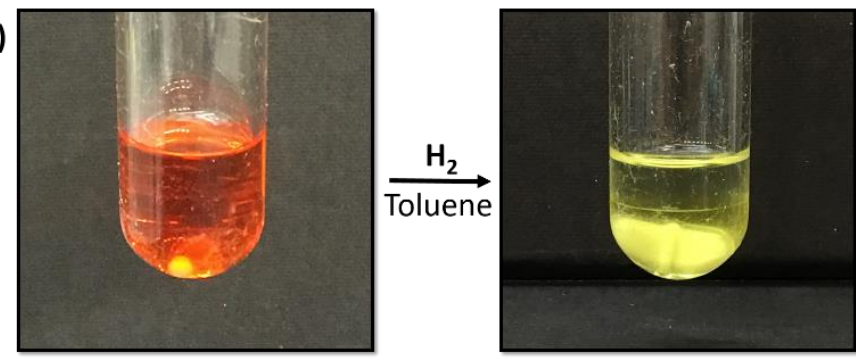

(B)
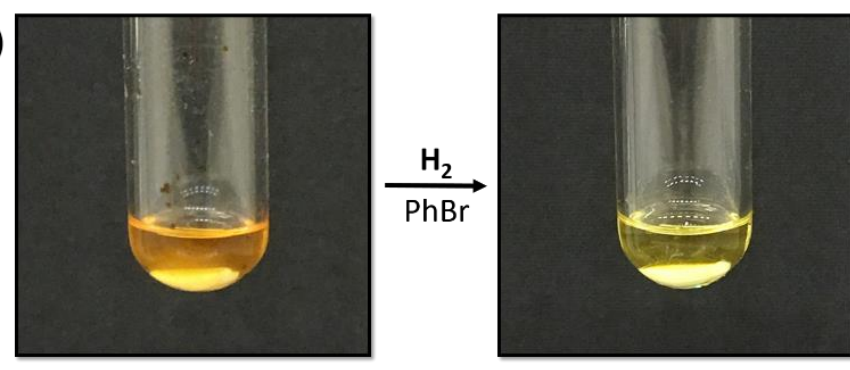

(C)

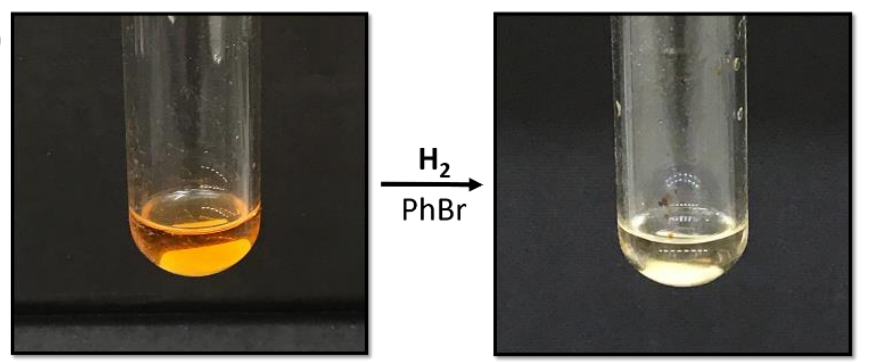

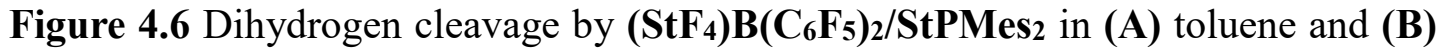
in bromobenzene, and (C) by BCF/StPMes2 in bromobenzene.

The product obtained from $\left(\mathrm{StF}_{4}\right) \mathbf{B}\left(\mathbf{C}_{6} \mathbf{F}_{5}\right)_{2} / \mathbf{S t P M e s}_{2}$ was purified by precipitation into hexane. A sticky yellow oil was obtained and solidified upon removal of solvent under vacuum. The resultant pale yellow solid was characterised by NMR spectroscopy, as shown below in Figure 4.7.

From the ${ }^{31} \mathrm{P}$ NMR spectrum, after introducing hydrogen gas to the FLP, the phosphorus was deshielded and gave two peaks at $-17.6 \mathrm{ppm}$ and $-17.9 \mathrm{ppm}$ respectively. Similar to the DEAD binding test described earlier, these two peaks should arise from the two isomers of the resultant product due to the asymmetric phosphine monomer. Attachment of protons was confirmed by proton-coupled ${ }^{31} \mathrm{P}$ 
NMR spectroscopy, which the ${ }^{1} J_{\mathrm{PH}}$ was determined as $485 \mathrm{~Hz}$. The ${ }^{11} \mathrm{~B}$ NMR spectrum revealed similar information. The boron was shielded and give two peaks at $-10.4 \mathrm{ppm}$ and $-13.1 \mathrm{ppm}$, corresponding to the two isomers of the product. The NMR spectra further confirmed the presumption of product isomerisation at elevated temperatures. The peaks in both the ${ }^{31} \mathrm{P}$ and ${ }^{11} \mathrm{~B}$ NMR spectra recombined, indicating the heatpromoted isomerisation exchange. It can be concluded from these spectra that the $\left(\mathrm{StF}_{4}\right) \mathbf{B}\left(\mathrm{C}_{6} \mathbf{F}_{5}\right)_{2} / \mathrm{StPMes}_{2}$ FLP system can afford dihydrogen cleavage. It should in

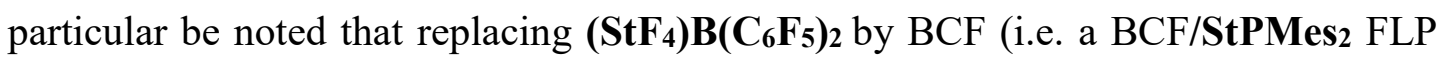
system) can lead to an even lower chemical shift at $-25.2 \mathrm{ppm}$ in the ${ }^{11} \mathrm{~B}$ NMR spectrum, indicating the slightly reduced Lewis acidity of $\left(\mathbf{S t F}_{4}\right) \mathbf{B}\left(\mathbf{C}_{6} \mathbf{F}_{5}\right)_{2}$ due to the incorporation of the vinyl group.

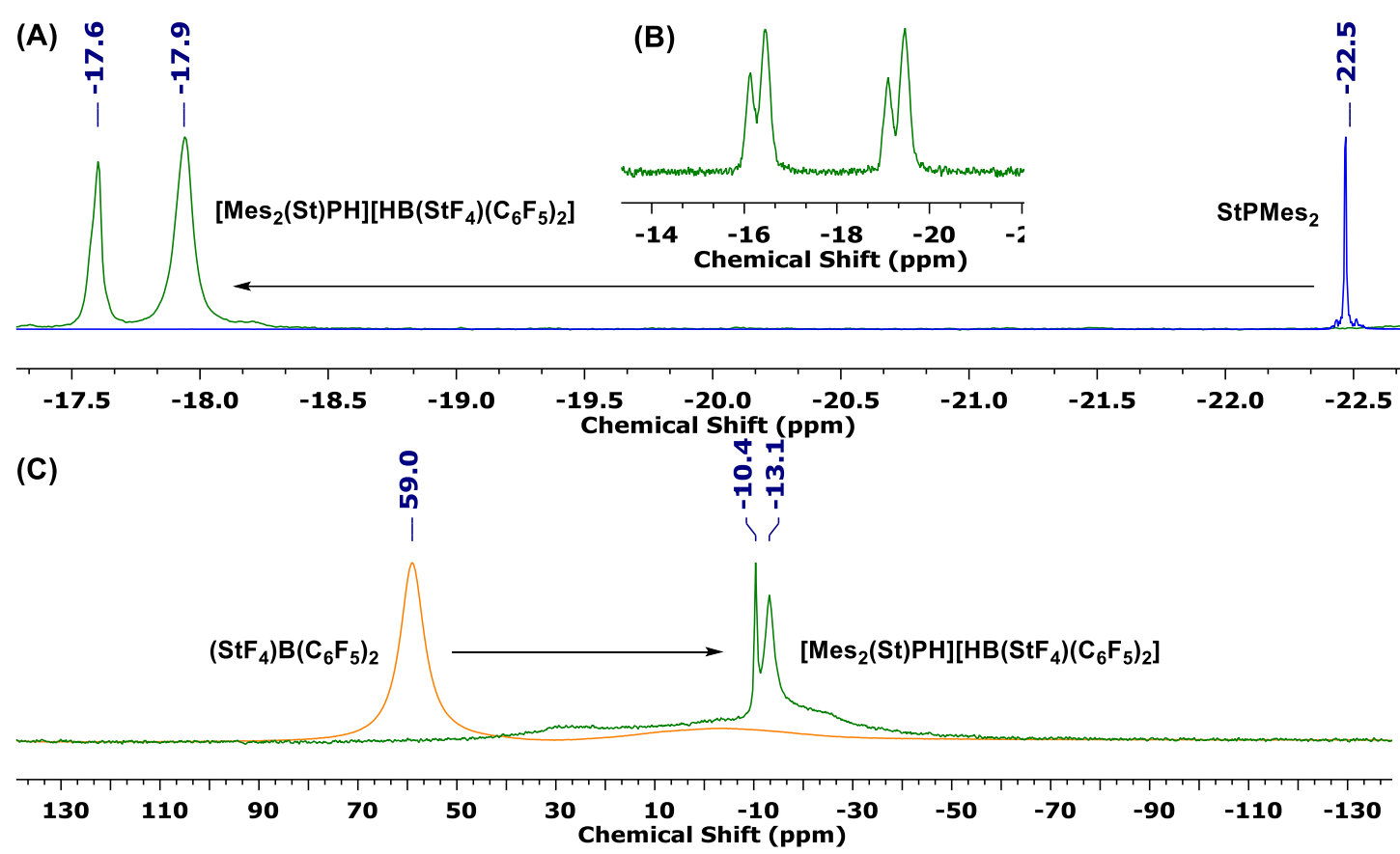

Figure 4.7 (A) ${ }^{31} \mathrm{P}\left\{{ }^{1} \mathrm{H}\right\}$, (B) ${ }^{1} \mathrm{H}$-coupled ${ }^{31} \mathrm{P}$, and (C) ${ }^{11} \mathrm{~B}\left\{{ }^{1} \mathrm{H}\right\}$ NMR spectra of StPMes2 (blue line) and $\left(\mathbf{S t F}_{4}\right) \mathbf{B}\left(\mathbf{C}_{6} \mathbf{F}_{5}\right)_{2}$ (orange line) and their product from dihydrogen cleavage (green line).

Another interesting feature of the dihydrogen cleavage by the $\left(\mathbf{S t F}_{4}\right) \mathbf{B}\left(\mathbf{C}_{6} \mathbf{F}_{5}\right)_{2} / \mathbf{S t P M e s}_{2}$ FLP system is its reversible nature. The product 
[Mes2(St)PH] $\left[\mathbf{H B}\left(\mathrm{StF}_{4}\right)\left(\mathrm{C}_{6} \mathbf{F}_{5}\right)_{2}\right]$ was characterised by NMR spectroscopy under one atmosphere of nitrogen at $100{ }^{\circ} \mathrm{C}$. Apart from the recombination of isomer peaks, as mentioned earlier, the free unbound phosphorus and boron-monomers were also observed from the spectra, shown below in Figure 4.8. By integral it was known that $16.4 \%$ of $\left[\mathbf{M e s}_{2}(\mathbf{S t}) \mathbf{P H}\right]\left[\mathbf{H B}\left(\mathbf{S t F}_{4}\right)\left(\mathbf{C}_{6} \mathbf{F}_{5}\right)_{2}\right]$ was converted back to the original FLP. This result indicated that our system is one of the very rare examples of reversible dihydrogen cleavage FLP systems, even though the conversion was not very efficient. A similar test was performed to BCF/StPMes2 FLP system, but no release of $\mathrm{H}_{2}$ was observed.

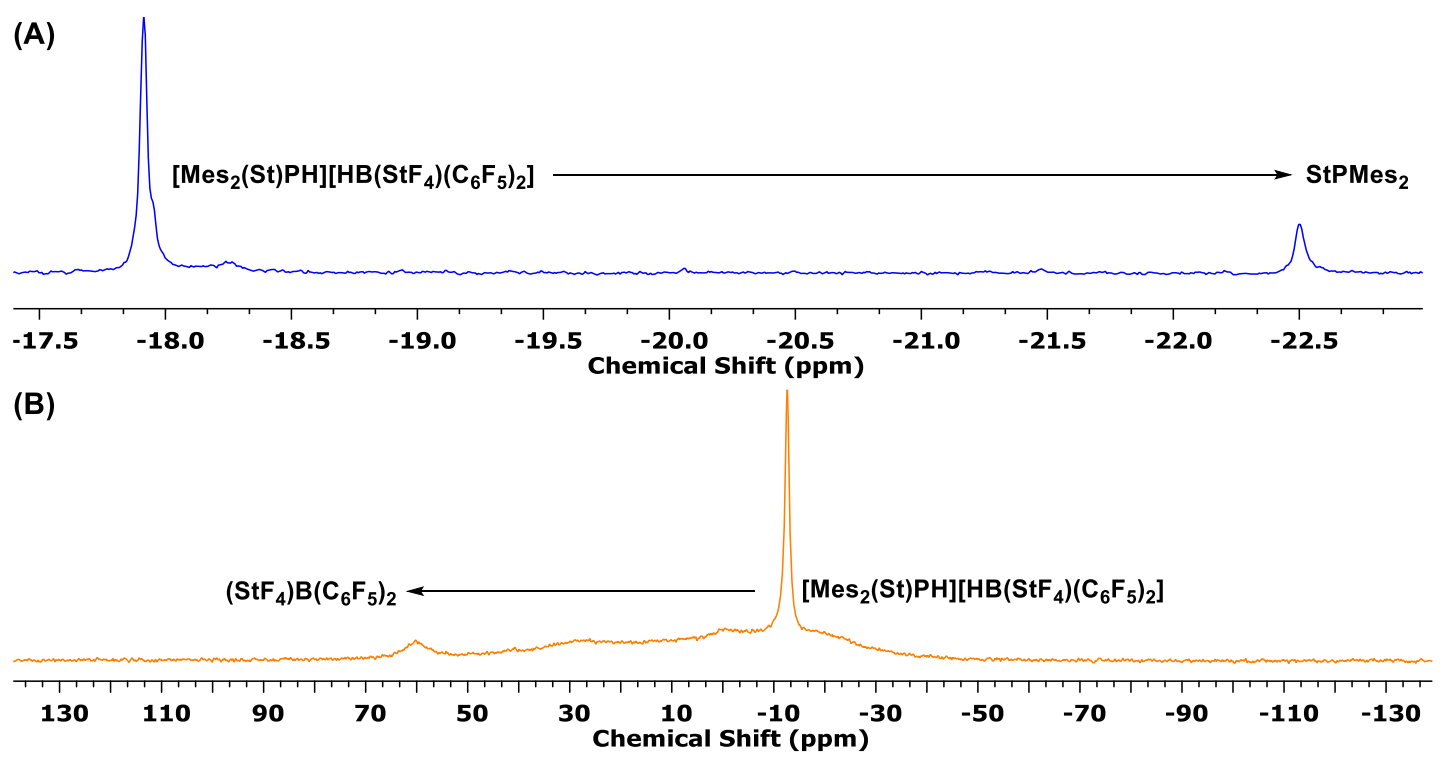

Figure 4.8 (A) ${ }^{31} \mathrm{P}$ and (B) ${ }^{11} \mathrm{~B}$ NMR spectra of $\left[\mathbf{M e s}_{2}(\mathbf{S t}) \mathbf{P H}\right]\left[\mathbf{H B}\left(\mathbf{S t F}_{4}\right)\left(\mathbf{C}_{6} \mathbf{F}_{5}\right)_{2}\right]$ at $100{ }^{\circ} \mathrm{C}$

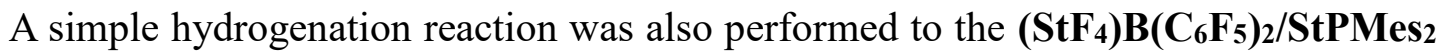
in order to test its catalysis reactivity. $5 \%$ in molarity of this FLP was added as a catalyst to N-benzylidene-tert-butylamine. The reaction was performed under one atmosphere of hydrogen gas at $80{ }^{\circ} \mathrm{C}$, as shown below in Scheme 4.5.

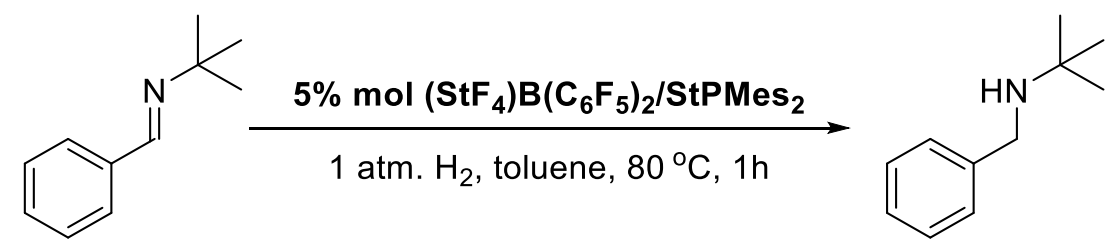

Scheme 4.5 Hydrogenation of N-benzylidene-tert-butylamine. 
After only one hour, the conversion reached $89.8 \%$, confirmed by the ${ }^{1} \mathrm{H}$ NMR spectrum. This FLP showed the excellent reactivity of the hydrogenation of this imine compound, which was very similar to other FLP systems using BCF. ${ }^{10}\left(\mathbf{S t F}_{4}\right) \mathbf{B}\left(\mathbf{C}_{6} \mathbf{F}_{5}\right)_{2}$ can also catalyse the reaction alone without the phosphorus monomer, as the imine itself can act as the base partner. As a result, a very similar conversion $(89.1 \%)$ was obtained, which was comparable to the examples of catalysis by BCF in the literature. ${ }^{11}$

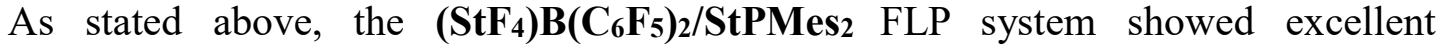
dihydrogen cleavage and hydrogenation reactivity. The system also showed its ability to capture carbon dioxide. An early stage test was performed by stirring $\left(\mathrm{StF}_{4}\right) \mathbf{B}\left(\mathrm{C}_{6} \mathbf{F}_{5}\right)_{2} / \mathrm{StPMes} 2$ under one atmosphere of $\mathrm{CO}_{2}$. After the reaction, the product was isolated by precipitation into hexane, and a yellow solid was resulted and characterised by NMR spectroscopy. The ${ }^{11} \mathrm{~B}$ NMR spectrum clearly showed a shielded sharp peak at $-1.9 \mathrm{ppm}$, corresponding to the tetra-coordinated carbon. The chemical shift was very similar to other FLP systems using BCF reported in the literature. ${ }^{12}$ The ${ }^{13} \mathrm{C}$ NMR showed a very weak quaternary carbon peak at $163.5 \mathrm{ppm}$, indicating the existence of the carbonyl group in the product. The ${ }^{31} \mathrm{P}$ NMR spectrum showed the existence of several impurities. The peaks at 46.6 and $46.4 \mathrm{ppm}$ were assigned to the desired product. Again, the two peaks arose from the two isomers of the resultant product, similar to the other binding tests mentioned before. Due to the time limitation of the project, only these early-stage results were obtained for the $\mathrm{CO}_{2}$ capture by this FLP system. The related research is still ongoing in our lab. Apart from dihydrogen and $\mathrm{CO}_{2},\left(\mathbf{S t F}_{4}\right) \mathbf{B}\left(\mathbf{C}_{6} \mathbf{F}_{5}\right)_{2} / \mathbf{S t P M e s} 2$ also proved reactive towards tert-butyl isocyanate, as confirmed by the ${ }^{11} \mathrm{~B}$ and ${ }^{31} \mathrm{P}$ NMR spectra. This system cannot activate alkenes, which were usually reactive towards FLPs with more basic phosphines $\left({ }^{t} \mathrm{Bu}_{3} \mathrm{P}\right.$ and $\mathrm{Mes}_{3} \mathrm{P}$ ). 
As a summary, the fully fluorinated aryl-substituted borane monomer $\left(\mathbf{S t F}_{4}\right) \mathbf{B}\left(\mathbf{C}_{6} \mathbf{F}_{5}\right)_{2}$ showed its excellent potential in making the next generation poly(FLP) gel. This boron monomer was able to activate various small molecules, including dihydrogen and $\mathrm{CO}_{2}$. Although the problems in polymerisation need to be worked out, the poly(FLP) gel made from the new boron-monomer showed great potential applications in heatresponsiveness and catalysis.

\subsection{Other Boron-containing Monomers}

Apart from the highly Lewis acidic monomer $\left(\mathbf{S t F}_{\mathbf{4}}\right) \mathbf{B}\left(\mathbf{C}_{\mathbf{6}} \mathbf{F}_{\mathbf{5}}\right)_{2}$ stated above, synthesis of some other boron-containing monomers via other synthetic routes were also explored. Some early-stage work on this is briefly introduced in the following sections.

\subsubsection{Boron-functionalised Monomers by Hydroboration.}

Hydroboration has been applied not only to generating organoboron compounds, ${ }^{13}$ but also germinal frustrated Lewis pairs. ${ }^{14}$ A hydroboration reaction to make another boron-containing monomer was attempted using di-vinyl functionalised styryl compound 4-(3-butenyl)styrene (3-BSt) and bis(pentafluorophenyl)borane $\left(\mathbf{H B}\left(\mathbf{C}_{6} \mathbf{F}_{5}\right) 2\right.$, known as Piers' borane $\left.{ }^{15-16}\right)$. The hydroboration reaction between the two compounds was probed by NMR spectroscopy, as shown below in Scheme 4.6.<smiles>C=CCCc1ccc(C=C)cc1</smiles>

3-BSt<smiles>Fc1c(F)c(F)c(BCc2c(F)c(F)c(F)c(F)c2F)c(F)c1F</smiles>
$\operatorname{HB}\left(C_{6} F_{5}\right)_{2}$<smiles>C=Cc1ccc(CCCCB(c2c(F)c(F)c(F)c(F)c2F)c2c(F)c(F)c(F)c(F)c2F)cc1</smiles>
$\operatorname{St}\left(\mathrm{CH}_{2}\right)_{4} \mathrm{~B}\left(\mathrm{C}_{6} \mathrm{~F}_{5}\right)_{2}$

Scheme 4.6 Hydroboration reaction to synthesis boron-containing monomer. 
The hydroboration occurred selectively at the aliphatic end of 3-BSt in an antiMarkovnikov manner. The reaction proceeded rapidly and precisely, and the NMR spectroscopy revealed no side-reactions. The ${ }^{1} \mathrm{H}$ NMR spectrum indicated the full conversion of Piers' borane since its broad proton peak disappeared. Similar information can also be obtained from the ${ }^{11} \mathrm{~B}$ NMR spectrum (Figure 4.9), which has no observable starting material. The chemical shift of the resultant product peak was $74.1 \mathrm{ppm}$, which was a typical value of diarylalkylborane and comparable to its analogues in the literature. ${ }^{16}$ Although the product was not isolated at this stage, it was expected to be easily purified due to its very distinct solubility to the starting material and the "click" nature of the reaction. This clean synthetic route provided another possible way to produce boron-functionalised monomers.

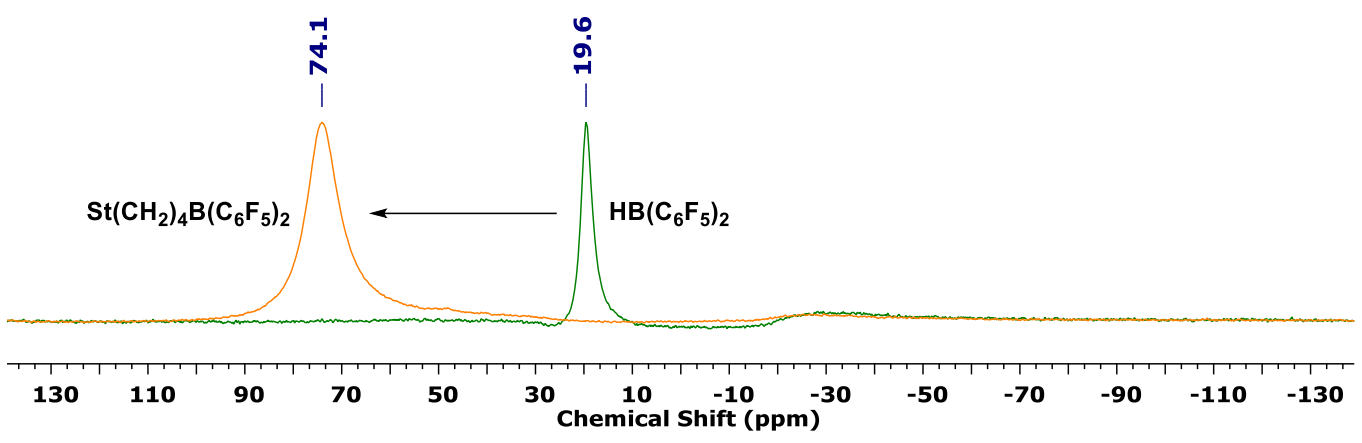

Figure 4.9 Overlapped ${ }^{11} \mathrm{~B}$ NMR spectrum to show the hydroboration reaction.

\subsubsection{Expansion of Coordination-Recovery Strategy}

Except for the boron compounds mentioned in Chapter Two, some other boroncontaining monomers were also attempted to be synthesised using the 2-APB analogues. Electron withdrawing trifluoromethyl groups were introduced at different positions of the aryl groups in these boron monomers. The structures of these targeted boron-monomers and their synthesis precursors (2-APB analogues) are shown below in Scheme 4.7. The para- and ortho-trifluoromethylphenyl substituted monomers $\mathbf{S t B}\left(4-\mathbf{C F}_{3}-\mathbf{P h}\right)_{2}$ and $\mathbf{S t B}\left(\mathbf{2}-\mathbf{C F}_{3}-\mathbf{P h}\right)_{2}$ had similar Lewis acidity and both were more 
acidic than the synthesised monomer StBPh2. The latter, however, had higher steric hindrance around the boron centre, leading to a potentially stronger resistance to hydrolysis. ${ }^{17}$ The last target boron-monomer $\mathbf{S t B}\left(\mathbf{3}, \mathbf{5}-\mathbf{C F}_{\mathbf{3}}-\mathbf{P h}\right)_{2}$ had the highest Lewis acidity. The bis(trifluoromethyl)phenyl group showed a similar ability to enhance the Lewis acidity of boron, as the pentafluorophenyl group. ${ }^{5}$ The Lewis acidity of $\mathbf{S t B}(\mathbf{3}, \mathbf{5}-$ $\left.\mathbf{C F}_{3}-\mathbf{P h}\right)_{2}$ was therefore expected to be similar to Yan et al.'s monomer.<smiles>C=Cc1ccc(B(c2ccc(C(F)(F)F)cc2)c2ccc(C(F)(F)F)cc2)cc1</smiles><smiles>FC(F)(F)c1ccc([B-]2(c3ccc(C(F)(F)F)cc3)NCCO2)cc1</smiles><smiles>C=Cc1ccc(B(c2ccccc2C(F)(F)F)c2ccccc2C(F)(F)F)cc1</smiles>
$\operatorname{StB}\left(2-\mathrm{CF}_{3}-\mathrm{Ph}\right)_{2}$<smiles>FC(F)(F)c1ccccc1B1OCCN1c1ccccc1C(F)(F)F</smiles>
2-APB $\left(2-\mathrm{CF}_{3}\right)$<smiles>C=Cc1ccc(B(c2cc(C(F)(F)F)cc(C(F)(F)F)c2)c2cc(C(F)(F)F)cc(C(F)(F)F)c2)cc1</smiles>

$\mathrm{StB}\left(3,5-\mathrm{CF}_{3}-\mathrm{Ph}\right)_{2}$<smiles></smiles>

2-APB(3,5- $\left.\mathrm{CF}_{3}\right)$

Scheme 4.7 Structures of trifluoromethyl-substituted triarylborane monomer and their precursors.

The 2-APB analogues were synthesised using a similar method to that used for the 2APB discussed previously. The synthesis was achieved by the nucleophilic substitution reaction between trialkyl borate and the Grignard reagents made from aryl halides, followed by the condensation reaction with ethanolamine. In the case of 2 $\mathbf{A P B}\left(4-\mathbf{C F}_{3}\right)$, since the Grignard reagent cannot be synthesised directly from its starting material $p$-bromobenzotrifluoride, organolithium was selected instead. The resultant products were easily purified and obtained as crystals. The NMR spectroscopy confirmed the structures of these products. 
The synthesis of $\mathbf{S t B}\left(\mathbf{4}-\mathbf{C F}_{3}-\mathbf{P h}\right)_{2}$ was first attempted by the Grignard reaction between StMgCl and 2-APB(4-CF $)$. The product $\mathbf{S t B}\left(4-\mathbf{C F}_{3}-\mathbf{P h}\right)_{2} \cdot \mathbf{N H}_{3}$ showed very clear peaks in the ${ }^{1} \mathrm{H},{ }^{11} \mathrm{~B}$ and ${ }^{19} \mathrm{~F}$ NMR spectra, confirming the presence of the ammonia protons, tetra-coordinated boron, and single fluorine environment. It was then treated with $\mathrm{HCl}$ etherate solution. A similar reaction phenomenon to that of $\mathbf{S t B P h} 2$ was observed in this case. The ${ }^{11} \mathrm{~B}$ NMR spectra were taken to monitor this process, as shown below in Figure 4.10.

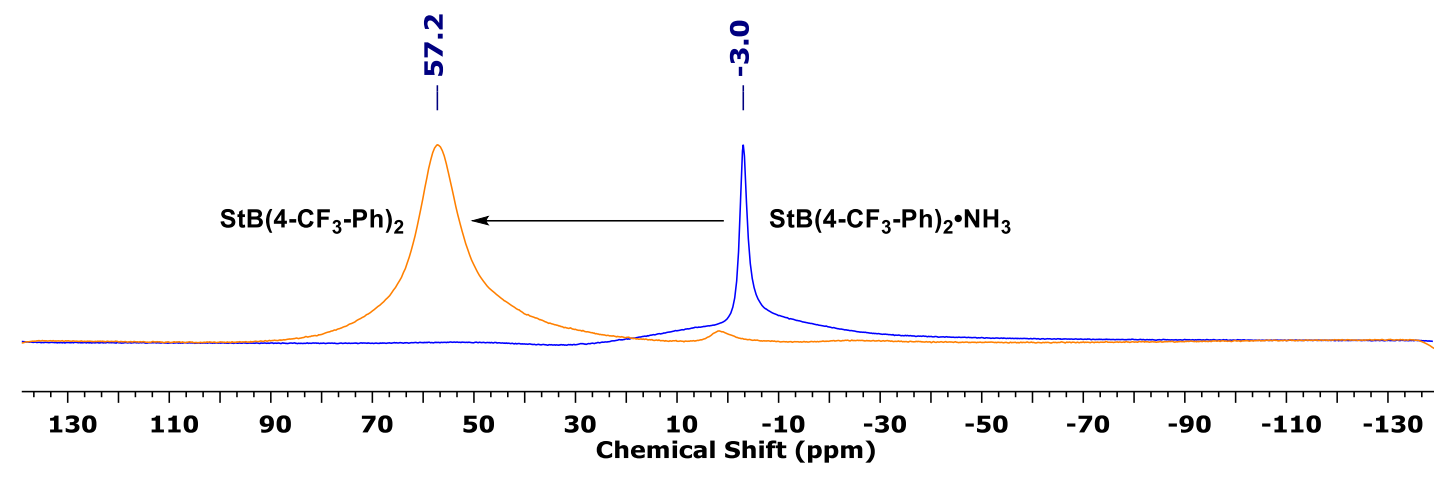

Figure 4.10 Overlapped ${ }^{11} \mathrm{~B}$ NMR spectra to monitor the reaction between $\mathrm{HCl}$ and $\mathrm{StB}\left(4-\mathrm{CF}_{3}-\mathrm{Ph}_{2}\right) \cdot \mathrm{NH}_{3}$.

An obvious deshielding of the boron peak was observed from -3.0 to $57.2 \mathrm{ppm}$ after the $\mathrm{HCl}$ treatment, indicating the successful removal of $\mathrm{NH}_{3}$ group. The relatively low chemical shift of the boron, however, suggested that it was coordinated by something else after the reaction. Several deshielded $\mathrm{Et}_{2} \mathrm{O}$ peaks were observed in the ${ }^{1} \mathrm{H}$ NMR spectrum, indicating that this was possibly the molecule that bound to the boron. Several side-reactions also seemed to occur due to the very small impurity peaks found in the ${ }^{1} \mathrm{H}$ NMR spectrum. To probe the role of $\mathrm{Et}_{2} \mathrm{O}$ in the ammonia-removal process, hexane could be used as an alternative reaction solvent, and $\mathrm{HCl}$ gas instead of its etherate solution. The attempted recrystallisation was also failed. The product cannot be recrystallised from $\mathrm{Et}_{2} \mathrm{O}$. When hexane was used, few white crystals can be seen from beginning, but soon settled to the bottom of the container as a yellow oil afterwards. The related research is still ongoing in our lab, however. 
The synthesis of $\mathbf{S t B}\left(\mathbf{2}-\mathbf{C F}_{3}-\mathbf{P h}\right)_{2} \cdot \mathbf{N H}_{3}$ was not successful. Its synthetic precursor 2APB(2-CF $)$ seemed too sterically hindered and did not allow the Grignard reagent to get close to the boron centre. No collectable product was obtained after the reaction. There were also problems with the synthesis of $\mathbf{S t B}\left(\mathbf{3}, \mathbf{5}-\mathbf{C F}_{3}-\mathbf{P h}\right)_{2} \cdot \mathbf{N H}_{3}$. It seemed the product obtained after the reaction was not stable enough in air since it decomposed during the work-up procedure. A potential solution was to add a donating agent before the Grignard quenching. Due to the time limitations of this project, however, the related synthesis is not yet complete and remains a subject of research in our lab.

\subsection{Conclusion}

A fully-fluorinated boron-containing monomer $\left(\mathbf{S t F}_{4}\right) \mathbf{B}\left(\mathbf{C}_{6} \mathbf{F}_{5}\right)_{2}$ was synthesised. It has the highest Lewis acidity ever published in the literature, which was very comparable to its analogue BCF. Confirmed by the NMR spectroscopy, this boron monomer cannot bind to the phosphorus-containing monomer StPMes2, hence forms an FLP with it. Upon mixing of these two monomers, a colour change was observed, indicating the weak interactions between the boron and phosphorus centres. This FLP proved reactive towards dihydrogen molecule. Apparent changes in chemical shifts of these two monomers in the ${ }^{11} \mathrm{~B}$ and ${ }^{31} \mathrm{P}$ NMR spectra were observed under hydrogen gas environment. The proton-coupled ${ }^{31} \mathrm{P}$ NMR spectrum confirmed the proton attachment on the phosphorus atom. The dihydrogen cleavage was fast, taking less than $30 \mathrm{~min}$ in an appropriate solvent. The dihydrogen cleavage was also reversible, with hydrogen gas was released upon heating. Efficient hydrogenation can also be achieved by this FLP. Apart from dihydrogen cleavage, this FLP also proved able to activate $\mathrm{CO}_{2}$ and some other small molecules.

The polymerisation of this highly Lewis acidic monomer is still work-in-progress. 
Early results, however, show that the polymerisation of this monomer suffered from its high Lewis acidity. The polymerisation reagents can bind to the boron atom, leading to a reduction in reactivity. Even though some copolymer samples were still synthesised by free radical polymerisations with significantly reduced yield. The new boron-incorporated copolymer was mixed with the aforementioned phosphoruscontaining copolymer poly(Styrene-co-StPMes2). The resultant mixture also gave a change in colour, indicating the formation of weak interactions between the boron and phosphorus. Instead of a polymer solution with high fluidity, the resultant mixture slowly turned to a non-covalently crosslinked gel. This new generation poly(FLP) gel showed its responsiveness to temperature. It reverted to a polymer solution when it was heated, and reformed as a gel once it was cooled down. The promising results from the small molecule activation and gelation experiments indicated that the new boron monomer $\left(\mathbf{S t F}_{4}\right) \mathbf{B}\left(\mathbf{C}_{6} \mathbf{F}_{5}\right)_{2}$ has a great potential in producing a new generation of stimuli-responsive polymeric frustrated Lewis pair systems.

Apart from $\left(\mathbf{S t F}_{4}\right) \mathbf{B}\left(\mathbf{C}_{6} \mathbf{F}_{5}\right)$ 2, attempts were made to synthesise some other boroncontaining monomers. Hydroboration using Piers' borane showed a convenient method of generating monomers with boron-moieties. The coordination-recovery synthetic strategy also showed potential to be applied in synthesising other boron compounds. Although only some early stage results were introduced, both methods showed they are capable of making boron-containing monomers with well-tuned functionality and Lewis acidity. 


\subsection{References}

[1] Chen, L.; Liu, R.; Yan, Q., Angew. Chem., Int. Ed. 2018, 57 (30), 9336-9340.

[2] Chen, L.; Liu, R.; Hao, X.; Yan, Q., Angew. Chem., Int. Ed. 2019, 58 (1), $264-$ 268.

[3] Liu, R.; Liu, X.; Ouyang, K.; Yan, Q., ACS Macro Letters 2019, 200-204.

[4] Bläsing, K.; Bresien, J.; Labbow, R.; Schulz, A.; Villinger, A., Angew. Chem., Int. Ed. 2018, 57 (29), 9170-9175.

[5] Sivaev, I. B.; Bregadze, V. I., Coord. Chem. Rev., 2014, 270, 75-88.

[6] Jiang, Y.; Hess, J.; Fox, T.; Berke, H., J. Am. Chem. Soc. 2010, 132 (51), 18233 18247.

[7] O'Reilly, R. K.; Shaver, M. P.; Gibson, V. C.; White, A. J. P., Macromolecules 2007, 40 (21), 7441-7452.

[8] Shaver, M. P.; Allan, L. E. N.; Rzepa, H. S.; Gibson, V. C., Angew. Chem., Int. Ed. 2006, 45 (8), 1241-1244.

[9] Frohn, H. J.; Franke, H.; Fritzen, P.; Bardin, V. V., J. Organomet. Chem. 2000, $598(1), 127-135$.

[10] Stephan, D. W.; Erker, G., Frustrated Lewis Pair Mediated Hydrogenations. In Frustrated Lewis Pairs I: Uncovering and Understanding, Erker, G.; Stephan, D. W., Eds. Springer Berlin Heidelberg: Berlin, Heidelberg, 2013; pp 85-110.

[11] Chase, P. A.; Jurca, T.; Stephan, D. W., Chem. Commun. 2008, (14), 17011703.

[12] Mömming, C. M.; Otten, E.; Kehr, G.; Fröhlich, R.; Grimme, S.; Stephan, D. W.; Erker, G., Angew. Chem., Int. Ed. 2009, 48 (36), 6643-6646.

[13] Brown, H. C., Tetrahedron 1961, 12 (3), 117-138.

[14] Spies, P.; Erker, G.; Kehr, G.; Bergander, K.; Fröhlich, R.; Grimme, S.; Stephan, D. W., Chem. Commun. 2007, (47), 5072-5074.

[15] Parks, D. J.; von H. Spence, R. E.; Piers, W. E., Angew. Chem. Int. Ed. Engl. 
1995, $34(7), 809-811$.

[16] Parks, D. J.; Piers, W. E.; Yap, G. P. A., Organometallics 1998, 17 (25), 54925503.

[17] Parab, K. K. Boron Containing Vinyl Aromatic Polymers: Synthesis, Characterization and Applications. Rutgers, The State University of New Jersey, May 2009. 


\section{Chapter Five. Conclusions and Future}

\section{Work}

The aim of this project was to develop a novel class of materials with frustrated Lewis pairs as the dynamic crosslinking. In Chapter Two, the detailed investigations on the synthesis of boron and phosphorus-containing monomers were introduced. Two different phosphorus-containing monomers StPPh2 and StPMes2 were synthesised. The former was synthesised for control experiments since it was less sterically hindered. It was expected to form CLP rather than FLP with the target boron-monomer. StPMes2, meanwhile, was more sterically hindered, so it was expected to be suitable for the aim of the project. Particular effort was put into the boron-monomer synthesis. The synthesis via exchange reactions did not prove successful due to the accumulated introduction of impurities and uncontrolled side-products. Instead, a novel synthetic strategy named coordination-recovery was used. The boron-containing intermediates 2-APB and $\mathbf{S t B P h}_{2} \cdot \mathbf{N H}_{3}$ in this strategy were amine-coordinated, which had unique solubility. This dramatically reduced the difficulty of purification and enabled successful synthesis. The coordinated amines were removed by treatment with $\mathrm{HCl}$ in order to recover the desired boron monomer StBPh2. All three synthesised monomers were copolymerised with styrene via RAFT polymerisation. The resultant copolymers were well-controlled in molecular weight and dispersity, showed the "living" character of the polymerisation.

In Chapter Three, gelation experiments using the synthesised copolymers were investigated. As expected, StPMes2 formed FLP with StBPh2, and their copolymers successfully formed the first generation polymeric FLP. Due to the steric encumbrance between the phosphorus and boron, gelation could not occur between the two 
copolymers until a bridging molecule DEAD was added. DEAD formed bridges between the boron and phosphorus, which was proven to be dynamic and exchangeable. This enabled the resultant polymer gel to be both heat-responsive and self-healing. These properties of the poly(FLP) gel were also investigated and confirmed by rheology, assisted by Utku Yolsal. Apart from this poly(FLP) system, the polymer gels made from $\mathbf{S t P P h} 2$ and $\mathbf{S t B P h} 2$ were also briefly introduced. The copolymers made from them could readily form poly(CLP) due to the weak binding between the boron and phosphorus. Nonetheless, the gelation could still be controlled by the protection of the boron centre using THF. The addition of DEAD allowed the generation of another Lewis pair containing-polymer gel, thus demonstrating a further insight into the process of synthesising stimuli-responsive polymer materials.

After the success of the first generation poly(FLP), the system was improved by modifying the boron-containing moieties. The research into synthesising this highly Lewis acidic boron-monomer was introduced in Chapter Four. $\left(\mathbf{S t F}_{4}\right) \mathbf{B}\left(\mathbf{C}_{6} \mathbf{F}_{5}\right)_{2}$ was successfully synthesised. The FLP formed by using it with StPMes2 showed great potential in dihydrogen cleavage and $\mathrm{CO}_{2}$ capture. The copolymers made from these two monomers also readily generated a supramolecular crosslinked polymer gel, which was thermal responsive. The polymerisation of this boron-monomer suffered from the high Lewis acidity, however, and the related research is still ongoing in our lab.

The work of this thesis has demonstrated the successful synthesis of heat-responsive self-healing polymeric networks from boron and phosphorus-containing macromolecules. This polymer gel is of significance as it complements the supramolecular polymer network with a new type of crosslinking. The novel coordination-recovery synthetic method for boron-containing monomers is also important since it provides a simple and efficient way to produce organoboron 
compounds. As the first generation poly(FLP) gel, the investigation on the solvent effect on the resultant gel behaviour is still lacking. As mentioned in Chapter Three, using a poor solvent like chloroform led to strong crosslinking and discouraged dynamic exchange. Therefore future research can focus on expanding the solvent system. This could be achieved either by preparing the gels using various solvents, or replacement of the toluene solvent after gelation. The mechanical and smart behaviours of the gels prepared using different solvent systems could be investigated. As a presumption, the gel should have reduced self-healing ability if a poor solvent like chloroform was used due to the inhibited crosslinking exchange, and higher temperature might be a significant factor to trigger the self-healing ability.

The continued research and development include the incomplete continuing work mentioned in previous chapters. In addition, the poly(FLP) can also be improved by incorporating more Lewis basic phosphine compounds. As stated above, incorporation of alkyl groups can enhance the $\sigma$-donating ability of phosphorus. Due to the less donating styryl group, however, the Lewis basicity will not be improved by simply replacing the mesitylene with alkyl groups such as cyclohexyl or tert-butyl groups. It is therefore necessary to introduce the methyl group at the ortho-positions (to phosphorus) of the styryl group. The resultant molecule is expected to be more basic and has some air-stability (Scheme 5.1). Another way to improve the poly(FLP) system would be to develop more air-stable boron monomers. Although the poly(FLP) gel gained some air-stability due to the coordination to boron, the gel can still be very slowly hydrolysed by air moisture during the crosslinking exchange and mechanical cracking. A method to overcome this limitation is to synthesise asymmetric boroncontaining monomers which contain a single steric group (Scheme 5.1). It was reported that incorporation of one mesitylene group onto the triarylborane could enhance the hydrolysis resistance by up to two weeks. The mesitylene group can also be modified to provide both the suitable electron-withdrawing effect and steric 
hindrance for the resultant boron centre.
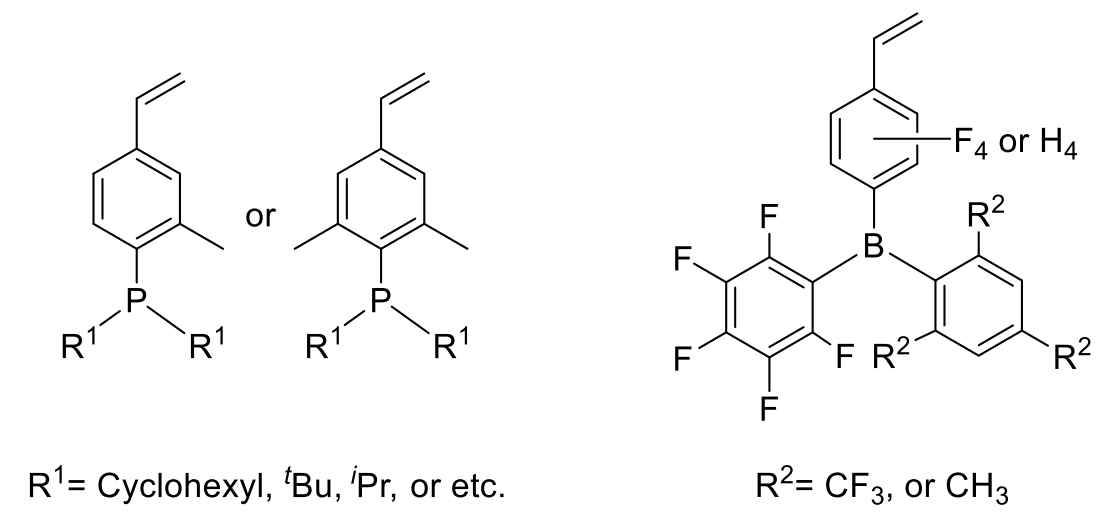

$\mathrm{R}^{2}=\mathrm{CF}_{3}$, or $\mathrm{CH}_{3}$

Scheme 5.1 Structure of the possible developed boron and phosphorus-containing monomers.

With the monomers mentioned above together with the monomers synthesised in this thesis in hand, it should be possible to give different combinations of FLPs which can activate either $\mathrm{CO}_{2}$ or $\mathrm{H}_{2}$, or even both. A poly(FLP) system containing more than one FLP should be possible to be synthesised, which allows sequentially capture and hydrogenation of trapped gas molecules (Scheme 5.2). The reduction reaction can be tested by feeding the $\mathrm{CO}_{2}$ and $\mathrm{H}_{2}$ gas sequentially or as a mixture. By altering the relative amount of $\mathrm{CO}_{2}$ and $\mathrm{H}_{2}$, varies $\mathrm{C}_{1}$ reduction product such as formic acid or methanol could be catalytically synthesised. The effect of additional reagents as proton sources to the hydrogenation can also be investigated. The polymerised FLPs have very different solubility parameters compared to other small molecules, meaning they are very easy to be separated from the products and recycled. This will reduce the overall cost of FLP catalysed hydrogenation reactions, leading to a huge commercial benefit. 


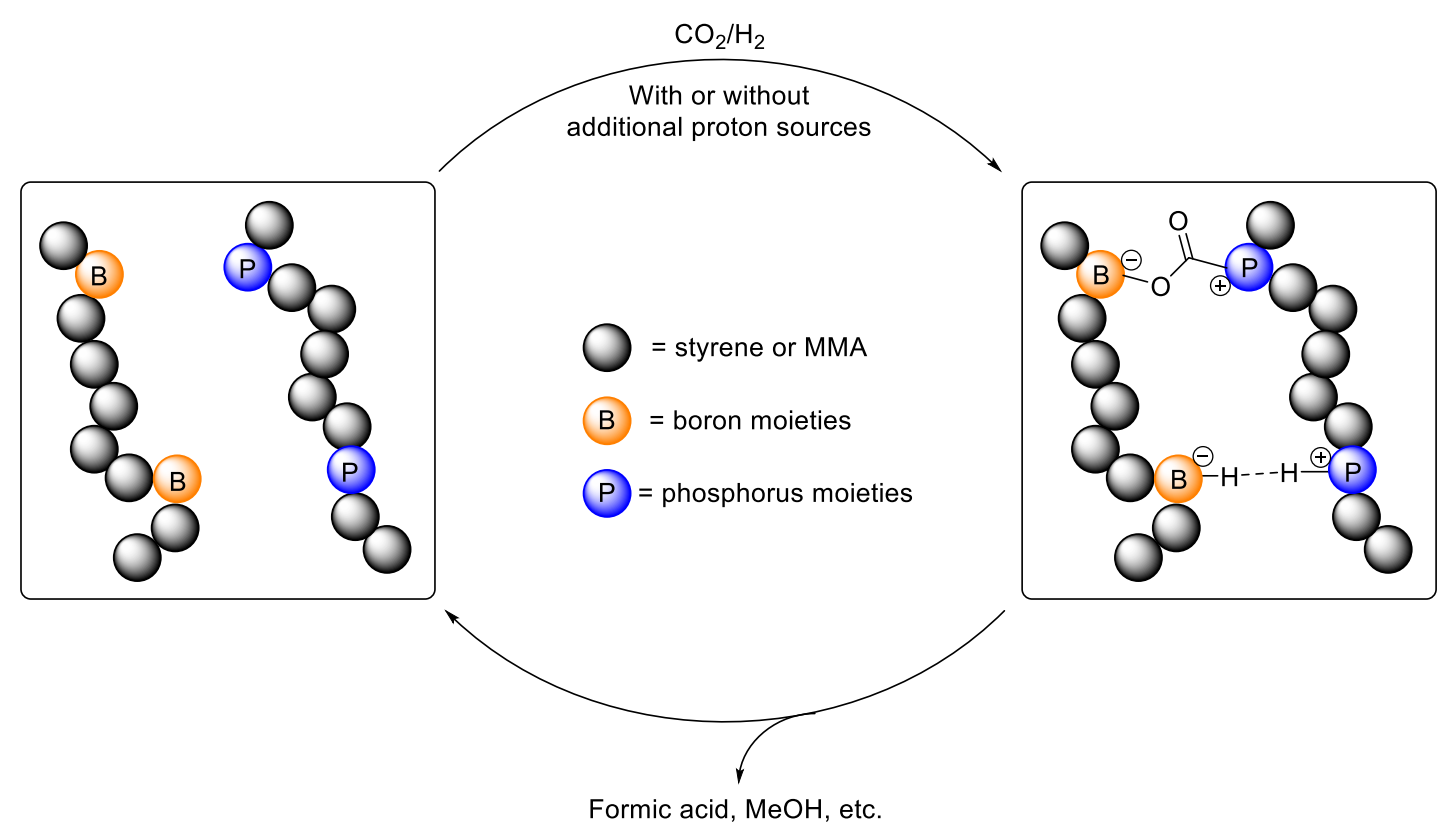

Scheme 5.2 Catalytic hydrogenation of $\mathrm{CO}_{2}$ using poly(FLP) system.

Apart from boron and phosphorus, other elements as Lewis centres for poly(FLP) are also important directions of exploration. Aluminium could be a good alternative choice of Lewis acid. It has a larger size compared to boron, leading to a more difficult steric protection. This often results in the formation of Masked FLP, meaning a Lewis base can bind to the aluminium moieties. The resultant FLP can still activate small molecules such as $\mathrm{CO}_{2}$ and $\mathrm{H}_{2}$, however. The high Lewis acidity of aluminium does not need the activation of strong electron withdrawing groups, meaning more versatile molecular designs. Therefore it could be predicted that a poly(FLP) using aluminium as the Lewis acid should have many interesting properties and responsive behaviours. For the Lewis base, nitrogen can be used instead of phosphorus. Nitrogen is smaller in size compared to phosphorus, hence it is more basic due to the higher electron density. It is more stable at lower coordination, meaning good air stability. N-heterocyclic carbenes (NHC) are also good choices for Lewis bases in poly(FLP). Polymeric based NHCs have been reported as a good polymeric Lewis base to bind with unique Lewis acid such as fullerene. The ultra-strong Lewis basicity of NHC should dramatically increase the overall reactivity of the poly(FLP) system. 


\section{Chapter Six. Experimental}

\subsection{General Considerations}

All reactions were performed, processed, purified and handled under inert atmosphere unless otherwise stated. All reactants, reagents and solvent were dried before use. The synthesis of monomers was performed using glovebox and Schlenk line techniques. Network gelation experiments and characterizations were done inside a glovebox as well, although were subsequently manipulated and processed outside of an inert atmosphere.

\subsection{Materials}

Commercial reagents were purchased from Acros Organics, Alfa Aesar, Fisher Scientific or Sigma Aldrich and used as received unless otherwise stated. 4chlorostyrene, 4-bromostyrene, methyl methacrylate, styrene, chlorodiphenylphosphine, 2-bromomesitylene, trimethylchlorosilane, and trimethylmethoxysilane were stirred over calcium hydride overnight, then distilled and degassed. Phosphorus trichloride was distilled under nitrogen and degassed before use. Magnesium was dried in an oven at $200{ }^{\circ} \mathrm{C}$ for at least 2 days before use. THF, dioxane and 1,2-dimethoxyethane were refluxed with sodium and benzophenone overnight and then distilled under nitrogen. Anhydrous DCM, toluene, and hexane were obtained from a Solvent Purification System, which containing alumina and copper catalysts. All the solvents were degassed prior to use by freeze-pump-thaw for at least three cycles. Anhydrous deuterated chloroform, deuterated toluene and deuterated dichloromethane were obtained by refluxing with calcium hydride, distilled under nitrogen, degassed and then storage under $3 \AA$ molecular sieves. Cumyl dithiobenzoate and 1,3,5-triisopropyl-2,4,6-dioxane were synthesized according to published 
procedures. ${ }^{1-2}$ Triphenylborane was synthesized using a similar synthetic route of 4styryl-diphenylborane.

\subsection{Instrumentations}

${ }^{1} \mathrm{H},{ }^{13} \mathrm{C},{ }^{31} \mathrm{P}$ and ${ }^{11} \mathrm{~B}$ NMR spectra were obtained from a $500 \mathrm{MHz}$ Bruker Avance NMR Instrument at ambient temperature. ${ }^{11} \mathrm{~B}$ NMR spectra were taken by either using a quartz or borosilicate glass Young's tap NMR tube, including background suppression when necessary. All $\mathrm{O}_{2}$ /moisture sensitive experiments were performed by using a glovebox, or Schlenk lines equipped with an in-line gas drying column containing a copper catalyst. Molecular weights of polymers were determined by a Malvern triple detection gel permission chromatography (GPC) instrument. The GPC was run with THF at a flow rate of $1.00 \mathrm{~mL} / \mathrm{min}$ at ambient temperature. Molecular weights obtained were relative to narrow dispersity polystyrene standards. Microstructural images of the polymer network were obtained by a Zeiss Ligma HDVP scanning electron microscopy (SEM) instrument. The SEM samples were prepared by freezedrying from benzene, then coated by gold. All glassware for air-sensitive reactions were pre-dried by an oven overnight. The physical properties of polymeric frustrated Lewis pair networks were analysed by a stress-controlled Anton Parr MCR320 rheometer with parallel plate geometry - $40 \mathrm{~mm}$ rotating top plate and $50 \mathrm{~mm}$ stationary bottom plate with roughened (crosshatched) geometry. 


\subsection{Synthesis of Lewis Base Compounds}

\subsubsection{Synthesis of StPPh2}<smiles>C=Cc1ccc(P(c2ccccc2)c2ccccc2)cc1</smiles>

Magnesium ( $0.131 \mathrm{~g}, 5.37 \mathrm{mmol})$ and a crystal of $\mathrm{I}_{2}$ was added into an ampoule. The system was purged and refilled with $\mathrm{N}_{2}$ gas. Anhydrous THF (10.0 mL) was added and the mixture was stirred at $0{ }^{\circ} \mathrm{C}$. 4-bromostyrene $(0.50 \mathrm{~mL}, 3.824 \mathrm{mmol})$ was added dropwise into the mixture at $0{ }^{\circ} \mathrm{C}$ over $30 \mathrm{~min}$. After completion of addition, the ice bath was removed, and the reaction mixture was stirred at r.t. for $4 \mathrm{~h}$. After the reaction was complete, the Grignard reagent solution was purified by cannula filtration to remove any unreacted magnesium. Chlorodiphenylphosphine $(0.70 \mathrm{~mL}, 3.824 \mathrm{mmol})$ was added dropwise to the Grignard solution at $-78^{\circ} \mathrm{C}$ over $15 \mathrm{~min}$. and the reaction mixture was then stirred at r.t. for $4 \mathrm{~h}$. Any remaining Grignard reagent was quenched by saturated $\mathrm{NH}_{4} \mathrm{Cl}$ aqueous solution. Then the mixture was extracted by $\mathrm{Et}_{2} \mathrm{O}(50 \mathrm{~mL} \times 3)$, and then washed by $\mathrm{H}_{2} \mathrm{O}(30$ $\mathrm{mL} \times 3$ ). The combined organic phase was dried by $\mathrm{MgSO}_{4}$ and the solvent was removed under vacuum. The crude product left was further purified by silica gel chromatography (4:1 hexane/DCM) to give 4-styryl-diphenylphosphine as a white solid. Yield: 0.775 g, $70.3 \%$, m.p. $79-84{ }^{\circ} \mathrm{C} ;{ }^{1} \mathrm{H}$ NMR (400 MHz, $\left.\mathrm{CDCl}_{3}\right) \delta$ 7.42-7.28 $(\mathrm{m}, 14 \mathrm{H}), 6.74(\mathrm{dd}, J=17.6,10.9 \mathrm{~Hz}, 1 \mathrm{H}), 5.81(\mathrm{dd}, J=17.6,0.9 \mathrm{~Hz}, 1 \mathrm{H}), 5.31(\mathrm{dd}$, $J=10.9,0.9 \mathrm{~Hz}, 1 \mathrm{H}) ;{ }^{13} \mathrm{C} \mathrm{NMR}\left(101 \mathrm{MHz}, \mathrm{CDCl}_{3}\right) \delta 138.0,137.1(\mathrm{~d}, J=10.3 \mathrm{~Hz})$, $136.6(\mathrm{~d}, J=10.3 \mathrm{~Hz}), 136.4,134.0$ (d, $J=19.5 \mathrm{~Hz}), 133.7$ (d, $J=19.5 \mathrm{~Hz}), 128.8$, $128.5(\mathrm{~d}, J=7.0 \mathrm{~Hz}), 126.3(\mathrm{~d}, J=7.1 \mathrm{~Hz}), 114.7 ;{ }^{31} \mathrm{P} \mathrm{NMR}\left(162 \mathrm{MHz}, \mathrm{CDCl}_{3}\right), \delta=$ -5.82; EI-MS, $m / z,\left(\mathrm{M}^{+}\right)$calcd. for $\mathrm{C}_{20} \mathrm{H}_{17} \mathrm{P}: 288.1$. Found: $288.1\left(\mathrm{M}^{+}\right), 289.1\left(\mathrm{MH}^{+}\right)$. 


\subsubsection{Synthesis of StPMes2}

\subsubsection{Synthesis of Mes $2 P X$}

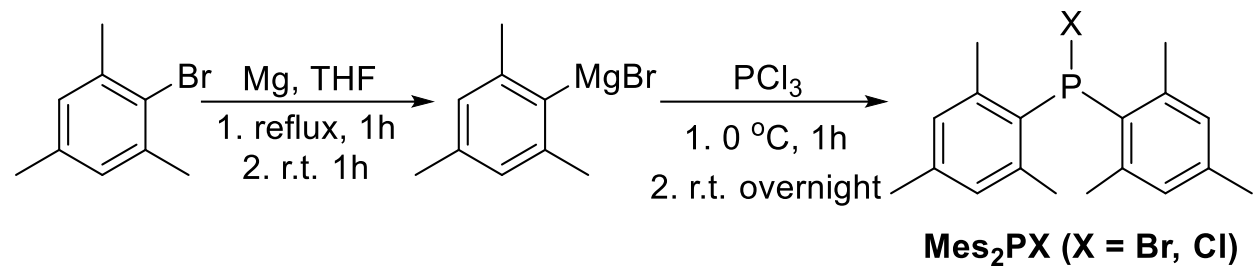

Dimesitylphosphorous halide was prepared by using reported literature with slight modifications. ${ }^{3-4}$ Under $\mathrm{N}_{2}$ atmosphere with stirring, 1,2-dibromoethane (1.0 mL, 11.6 mmol) was added dropwise into a mixture of magnesium turnings (5.77 g, $237 \mathrm{mmol})$ and anhydrous THF $(200 \mathrm{~mL})$. The mixture was stirred for $30 \mathrm{~min}$, then 2bromomesitylene $(30 \mathrm{~mL}, 196 \mathrm{mmol})$ was added into the mixture slowly, maintaining gentle reflux. After complete addition, the reaction mixture was refluxed for a further $1 \mathrm{~h}$. Then it was allowed to return to r.t. and stirred for another $3 \mathrm{~h}$. The Grignard reagent solution prepared, MesMgBr, was isolated by cannula filtration.

Phosphorous trichloride $(8.56 \mathrm{~mL}, 98.1 \mathrm{mmol})$ was dissolved in anhydrous THF (77 $\mathrm{mL}$ ), cooled to $-78^{\circ} \mathrm{C}$. The previously prepared Grignard solution, MesMgBr, was added dropwise at this temperature over $30 \mathrm{~min}$. Then the mixture was allowed to warm to r.t. and stirred overnight. The solvent was removed under reduced pressure, and the product was extracted by anhydrous hexane $(100 \mathrm{~mL} \times 4)$. The hexane solvent was removed under reduced pressure and the impurities were removed under vacuum. The product, dimesitylphosphorous bromide and dimesitylphosphorous chloride, were obtained as a pale yellow/white powder; Yield: 21.8 g, $70.5 \%$, m.p.62$67{ }^{\circ} \mathrm{C} ;{ }^{1} \mathrm{H}$ NMR $\left(500 \mathrm{MHz}, \mathrm{CDCl}_{3}\right), \delta 6.82(\mathrm{~d}, J=3.3 \mathrm{~Hz}, 4 \mathrm{H}), 2.35(\mathrm{t}, J=2.6 \mathrm{~Hz}$, 12H), $2.27(\mathrm{~d}, J=3.3 \mathrm{~Hz}, 6 \mathrm{H}) ;{ }^{31} \mathrm{P} \mathrm{NMR}\left(203 \mathrm{MHz}, \mathrm{CDCl}_{3}\right), \delta 85.2\left(\mathrm{~s}, \mathrm{Mes}_{2} \mathrm{PCl}\right), 74.8$ $\left(\mathrm{s}, \mathrm{Mes}_{2} \mathrm{PBr}\right)$. 


\subsubsection{Synthesis of StPMes2}<smiles>C=Cc1ccc(P(c2c(C)cc(C)cc2C)c2c(C)cc(C)cc2C)cc1</smiles>

for $1 \mathrm{~h}$ to give a Grignard solution, StMgCl.

The calculated amount by NMR of dimesitylphosphorous halide mixture (13.2 g, 38.4 mmol), was dissolved into anhydrous THF $(100 \mathrm{~mL})$, and the previously prepared Grignard solution, StMgCl, was added dropwise at $0{ }^{\circ} \mathrm{C}$. The reaction mixture was stirred at this temperature for $1 \mathrm{~h}$, then at r.t. overnight. Any unreacted Grignard reagent was quenched by addition of a saturated $\mathrm{NH}_{4} \mathrm{Cl}$ solution. The product was extracted by $\mathrm{Et}_{2} \mathrm{O}(50 \mathrm{~mL} \times 3)$ and then washed by $\mathrm{H}_{2} \mathrm{O}(20 \mathrm{~mL} \times 3)$. The organic phase was combined and dried over $\mathrm{MgSO}_{4}$. The solution was concentrated under reduced pressure. The resultant sticky oil was added dropwise into methanol to give a crude solid. To remove oxidised by-product, the crude was flushed through $\mathrm{Al}_{2} \mathrm{O}_{3}$ plug by hexane. The product, 4-styryl-dimeistylphosphine, was obtained as a white solid. Yield: 7.85 g, $53.2 \%$, m.p. $144-147{ }^{\circ} \mathrm{C} ;{ }^{1} \mathrm{H}$ NMR $\left(500 \mathrm{MHz}, \mathrm{CDCl}_{3}\right), \delta$ 7.41-7.28 (m, 4H), $6.83(\mathrm{~d}, J=3.1 \mathrm{~Hz}, 4 \mathrm{H}), 6.69(\mathrm{dd}, J=17.6,10.9 \mathrm{~Hz}, 1 \mathrm{H}), 5.76(\mathrm{dd}, J=17.5,0.8 \mathrm{~Hz}$, 1H), $5.25(\mathrm{~d}, J=10.9 \mathrm{~Hz}, 1 \mathrm{H}), 2.27(\mathrm{~s}, 6 \mathrm{H}), 2.11(\mathrm{~s}, 12 \mathrm{H}) ;{ }^{13} \mathrm{C} \mathrm{NMR}\left(126 \mathrm{MHz}, \mathrm{CDCl}_{3}\right)$ $\delta 143.0(\mathrm{~d}, J=15.9 \mathrm{~Hz}), 138.3,137.5(\mathrm{~d}, J=12.0 \mathrm{~Hz}), 137.1,136.7,133.9(\mathrm{~d}, J=22.4$ Hz), $130.2(\mathrm{~d}, J=18.8 \mathrm{~Hz}), 130.1(\mathrm{~d}, J=3.5 \mathrm{~Hz}), 126.1(\mathrm{~d}, J=6.8 \mathrm{~Hz}), 114.1,23.2$ (d, $J=16.0 \mathrm{~Hz}), 21.1 ;{ }^{31} \mathrm{P}$ NMR $\left(203 \mathrm{MHz}, \mathrm{CDCl}_{3}\right), \delta$-22.5; EI-MS, $m / z,\left(\mathrm{M}^{+}\right)$calcd. for $\mathrm{C}_{26} \mathrm{H}_{29} \mathrm{P}: 372.5$. Found: 372.2 . 


\subsubsection{Synthesis of PhPMes2}

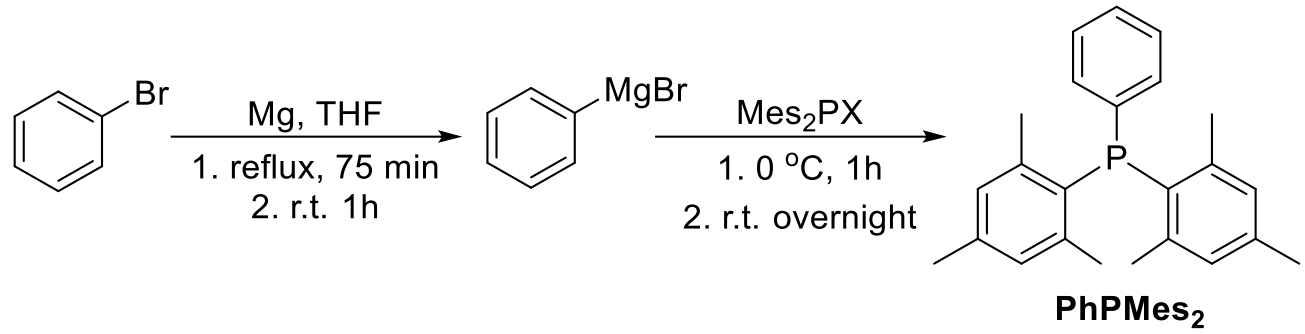

This compound was prepared via a different synthetic route compared to those published in the literature. Under an $\mathrm{N}_{2}$ atmosphere, 1,2-dibromoethane $(0.12 \mathrm{~mL}, 1.39$ mmol) was added into a mixture of magnesium turnings $(0.40 \mathrm{~g}, 16.5 \mathrm{mmol})$ and anhydrous THF (28 mL). The mixture was stirred for $30 \mathrm{~min}$, and then bromobenzene (2.14 g, $13.6 \mathrm{mmol})$ was added dropwise in $45 \mathrm{~min}$. After addition, the mixture was further refluxed for $30 \mathrm{~min}$ and then stirred at r.t. for $1 \mathrm{~h}$ to give a grey-black Grignard solution. This Grignard solution was dropwise cannula transferred into a THF solution $(40 \mathrm{~mL})$ of dimesitylphosphorous halide $(3.60 \mathrm{~g}, 11.4 \mathrm{mmol})$ prepared before at $0{ }^{\circ} \mathrm{C}$. After addition, the mixture was allowed to warm back to r.t. and stirred overnight. The reaction was then quenched by addition of saturated $\mathrm{NH}_{4} \mathrm{Cl}$ aqueous solution. The aqueous phase was extracted by $\mathrm{Et}_{2} \mathrm{O}(50 \mathrm{~mL} \times 3)$ and the combined organic phase was dried over $\mathrm{MgSO}_{4}$. After removal of solvent under reduced pressure, the crude product was purified by column chromatography (hexane) to give a white solid. Yield: 1.60 g, 40.6 \% m.p. 104-107 ${ }^{\circ} \mathrm{C} ;{ }^{1} \mathrm{H}$ NMR (400 MHz, $\left.\mathrm{CDCl}_{3}\right), \delta$ 7.44-7.34 (m, 2H), 7.33-7.23 (m, 3H) $6.86(\mathrm{~d}, J=2.9 \mathrm{~Hz}, 4 \mathrm{H}), 2.30(\mathrm{~s}, 6 \mathrm{H}), 2.14(\mathrm{~s}, 12 \mathrm{H}) ;{ }^{13} \mathrm{C}$ NMR $(126$ $\left.\mathrm{MHz}, \mathrm{CDCl}_{3}\right) \delta 143.0(\mathrm{~d}, \mathrm{~J}=15.6 \mathrm{~Hz}), 138.2,137.8(\mathrm{~d}, J=12.0 \mathrm{~Hz}), 133.8(\mathrm{~d}, J=$ $22.1 \mathrm{~Hz}), 130.3(\mathrm{~d}, J=18.9 \mathrm{~Hz}), 130.1(\mathrm{~d}, J=3.5 \mathrm{~Hz}), 128.2(\mathrm{~d}, J=6.8 \mathrm{~Hz}), 127.90$, $23.16(\mathrm{~d}, J=16.0 \mathrm{~Hz}), 21.06 ;{ }^{31} \mathrm{P} \mathrm{NMR}\left(162 \mathrm{MHz}, \mathrm{CDCl}_{3}\right), \delta-22.1$. 


\subsection{Synthesis of Lewis Acid Compounds}

\subsubsection{Synthesis of 2-APB analogues}

\subsubsection{Synthesis of 2-APB}

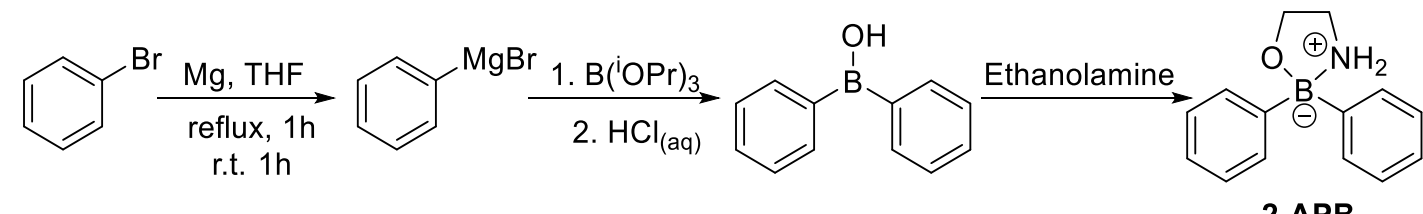

2-APB

The synthesis was modified from previous literature reports with slight modifications. ${ }^{5}$ Under $\mathrm{N}_{2}$ atmosphere with stirring, 1,2-dibromoethane $(0.7 \mathrm{~mL}, 8.12 \mathrm{mmol})$ was added dropwise into a mixture of magnesium turnings (5.46 g, $225 \mathrm{mmol}$ ) and anhydrous THF $(200 \mathrm{~mL})$. The mixture was stirred for $30 \mathrm{~min}$, then bromobenzene $(18.8 \mathrm{~mL}, 179 \mathrm{mmol})$ was added into the mixture slowly over $1 \mathrm{~h}$ under refluxing. The mixture then was allowed to return to r.t. and stirred for another one hour to give a dark grey/black Grignard solution. At $-78{ }^{\circ} \mathrm{C}$, the Grignard solution prepared was filtered cannula transferred dropwise into a THF solution of triisopropyl borate (20.6 $\mathrm{mL}, 89.3 \mathrm{mmol}$ ). After addition, the mixture was allowed to slowly return to r.t. and stirred overnight. $1 \mathrm{M}$ hydrochloric acid $(200 \mathrm{~mL})$ was added to quench the reaction. The organic phase was collected, and the aqueous phase was extracted using ethyl acetate $(100 \mathrm{~mL} \times 3)$. All organic phase fractions were combined and dried over $\mathrm{MgSO}_{4}$. The solution was concentrated under reduced pressure, then ethanolamine (9 $\mathrm{mL}, 149 \mathrm{mmol}$ ) was added. The mixture was stirred at r.t. overnight. After that the reaction mixture was diluted by ethyl acetate to re-dissolve all precipitated product, then the solution was washed with water and brine. The combined organic phase was dried over $\mathrm{MgSO}_{4}$, then the solution was concentrated under reduced pressure and precipitated into a mixture of chloroform and hexane (1:1) to give a colourless crystal. Yield: 15.6 g, 77.4\%; m.p. 185-190 ${ }^{\circ} \mathrm{C}$; ${ }^{1} \mathrm{H}$ NMR (500.2 MHz, DMSO-d6) $\delta 7.42-$ $7.37(\mathrm{~m}, 4 \mathrm{H}), 7.15-7.10(\mathrm{~m}, 4 \mathrm{H}), 7.05-7.00(\mathrm{~m}, 2 \mathrm{H}), 6.04(\mathrm{~s}, 2 \mathrm{H}), 3.77$ (t, $J=6.5$ 
$\mathrm{Hz}, 2 \mathrm{H}), 2.83(\mathrm{tt}, J=6.4 \mathrm{~Hz}, 2 \mathrm{H}) ;{ }^{13} \mathrm{C} \mathrm{NMR}\left(125.8 \mathrm{MHz}, \mathrm{DMSO}-d_{6}\right) \delta 131.97,127.05$, 125.33, 62.88, 41.83; ${ }^{11} \mathrm{~B}$ NMR (160.5 MHz, DMSO- $\left.d_{6}\right) \delta$ 4.5; EI-MS, m/z, (M+) calcd. for $\mathrm{C}_{14} \mathrm{H}_{16} \mathrm{BNO}$ : 225.1. Found: 225.1.

\subsubsection{Synthesis of 2-APB(4-CF $)$}

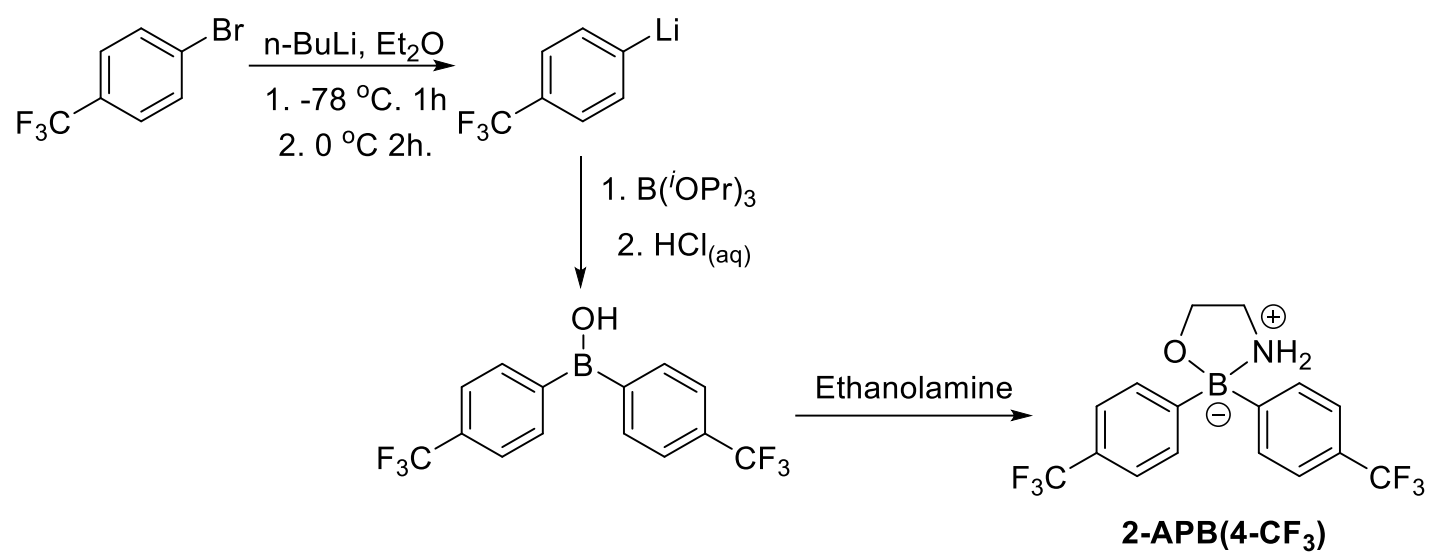

This unknown compound was prepared using the modified published procedures of synthesising 2-ABP class molecules. ${ }^{5}$ Under $\mathrm{N}_{2}$ atmosphere, 4-bromobenzotrifluoride (7.03 $\mathrm{mL}, 50.2 \mathrm{mmol})$ was dissolved in $\mathrm{Et}_{2} \mathrm{O}(115 \mathrm{~mL})$ and the solution was cooled to $-78{ }^{\circ} \mathrm{C} . \mathrm{n}-\mathrm{BuLi}(1.6 \mathrm{M}, 62.5 \mathrm{mmol})$ was added at this temperature dropwise over $1 \mathrm{~h}$, and the resultant organolithium solution was further stirred at $0{ }^{\circ} \mathrm{C}$ for another $2 \mathrm{~h}$. Then it was cooled down again to $-78^{\circ} \mathrm{C}$ and dropwise cannula transferred to ether (100 $\mathrm{mL})$ solution of triisopropyl borate $(5.17 \mathrm{~mL}, 22.4 \mathrm{mmol})$. The solution was allowed to turn to r.t. slowly and stirred overnight. $1 \mathrm{M}$ hydrochloric acid $(100 \mathrm{~mL})$ was added to quench the reaction. The product was extracted by diethyl ether $(100 \mathrm{~mL} \times 3)$ and the combined organic phase was dried over $\mathrm{MgSO}_{4}$. After removal of solvent, the solid left was re-dissolved in 3:1 EtOH: $\mathrm{H}_{2} \mathrm{O}(30 \mathrm{~mL})$, and ethanolamine $(2.25 \mathrm{~mL}, 37.3$ mmol) was added dropwise at r.t. The mixture was stirred at r.t. for $1 \mathrm{~h}$ and then heated up to $50{ }^{\circ} \mathrm{C}$ for another $1 \mathrm{~h}$. Any precipitated solid was re-dissolved by adding solvent and the organic phase was washed with brine water and dried over $\mathrm{MgSO}_{4}$. After removal of solvent, the product was purified by dissolving in diethyl ether followed 
by precipitation into hexane. The product was obtained as a colourless needle. Yield: 5.20 g, 64.3\%; ${ }^{1} \mathrm{H}$ NMR (500 MHz, DMSO- $\left.d_{6}\right) \delta 7.61$ (m, 4H), 7.48 (m, 4H), 6.35 (s, br, 2H), 3.79 (t, $J=6.5 \mathrm{~Hz}, 2 \mathrm{H}), 2.87$ (p, $J=6.4 \mathrm{~Hz}, 2 \mathrm{H}) ;{ }^{19} \mathrm{~F}$ NMR (471 MHz, DMSO$\left.d_{6}\right) \delta-60.6 ;{ }^{11} \mathrm{~B}$ NMR $\left(160 \mathrm{MHz}, \mathrm{DMSO}-d_{6}\right) \delta 3.7$.

\subsubsection{Synthesis of 2-APB $\left(2-\mathrm{CF}_{3}\right)$}
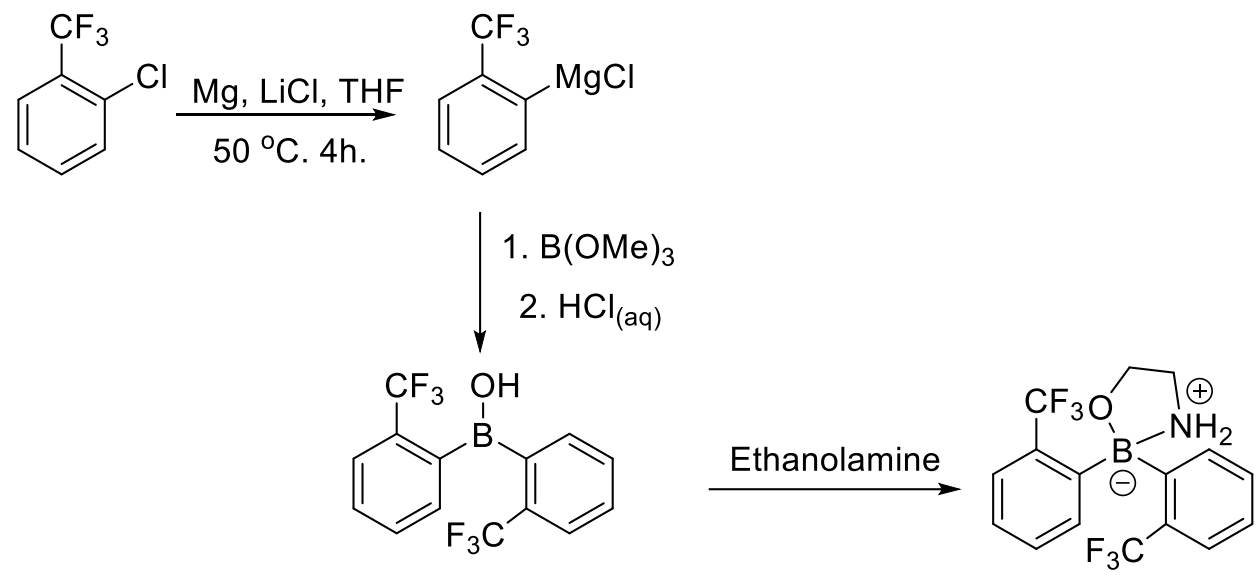

2-APB $\left(2-\mathrm{CF}_{3}\right)$

This unknown compound was synthesised according to the modified published procedures of synthesising 2-ABP class molecules. ${ }^{5}$ Under inert atmosphere, bromoethane $(0.45 \mathrm{~mL}, 6.03 \mathrm{mmol})$ was added into a mixture of THF $(100 \mathrm{~mL})$, magnesium (2.28 g, $93.8 \mathrm{mmol})$ and lithium chloride (1.15 g, $27.1 \mathrm{mmol})$. The mixture was refluxed for at least $30 \mathrm{~min}$. Then 2-chlorobenzotrifluoride (11.8 mL, $90.1 \mathrm{mmol})$ was added dropwise at $50{ }^{\circ} \mathrm{C}$ over $1 \mathrm{~h}$. After addition, the mixture was stirred at this temperature for $3 \mathrm{~h}$. The resultant Grignard solution was dropwise cannula transferred into the THF $(100 \mathrm{~mL})$ solution of trimethyl borate $(5 \mathrm{~mL}, 44.8 \mathrm{mmol})$ at $-78^{\circ} \mathrm{C}$. Then the mixture was allowed to warm back to r.t. and stirred overnight. $1 \mathrm{M}$ hydrochloric acid $(100 \mathrm{~mL})$ was added to quench any unreacted Grignard. The product was extracted using diethyl ether $(50 \mathrm{~mL} \times 3)$ and the combined organic phase was dried over $\mathrm{MgSO}_{4}$. The solvent was concentrated under reduced pressure and ethanolamine (4.5 mL, $74.6 \mathrm{mmol}$ ) was added dropwise at r.t. The mixture was stirred overnight. 
Any precipitated solid was re-dissolved and the organic phase was washed by brine water. After removal of solvent, the product was purified by recrystallisation from diethyl ether to give colourless crystals. Yield: $6.82 \mathrm{~g}, 42.2 \%$. ${ }^{1} \mathrm{H}$ NMR (500 MHz, DMSO- $\left.d_{6}\right) \delta 7.85(\mathrm{~d}, J=7.5 \mathrm{~Hz}, 2 \mathrm{H}), 7.43(\mathrm{~m}, 4 \mathrm{H}), 7.27(\mathrm{td}, J=7.5,1.3 \mathrm{~Hz}, 2 \mathrm{H}), 6.23$ $(\mathrm{t}, J=6.1 \mathrm{~Hz}, 2 \mathrm{H}), 3.69$ (t, $J=6.3 \mathrm{~Hz}, 2 \mathrm{H}), 2.87$ (p, $J=6.2 \mathrm{~Hz}, 2 \mathrm{H}) ;{ }^{19} \mathrm{~F}$ NMR (471 $\left.\mathrm{MHz}, \mathrm{DMSO}-d_{6}\right) \delta-55.6 ;{ }^{11} \mathrm{~B}$ NMR $\left(160 \mathrm{MHz}, \mathrm{DMSO}-d_{6}\right) \delta 4.4$.

\subsubsection{Synthesis of 2-APB(3,5-CF 3$)$}

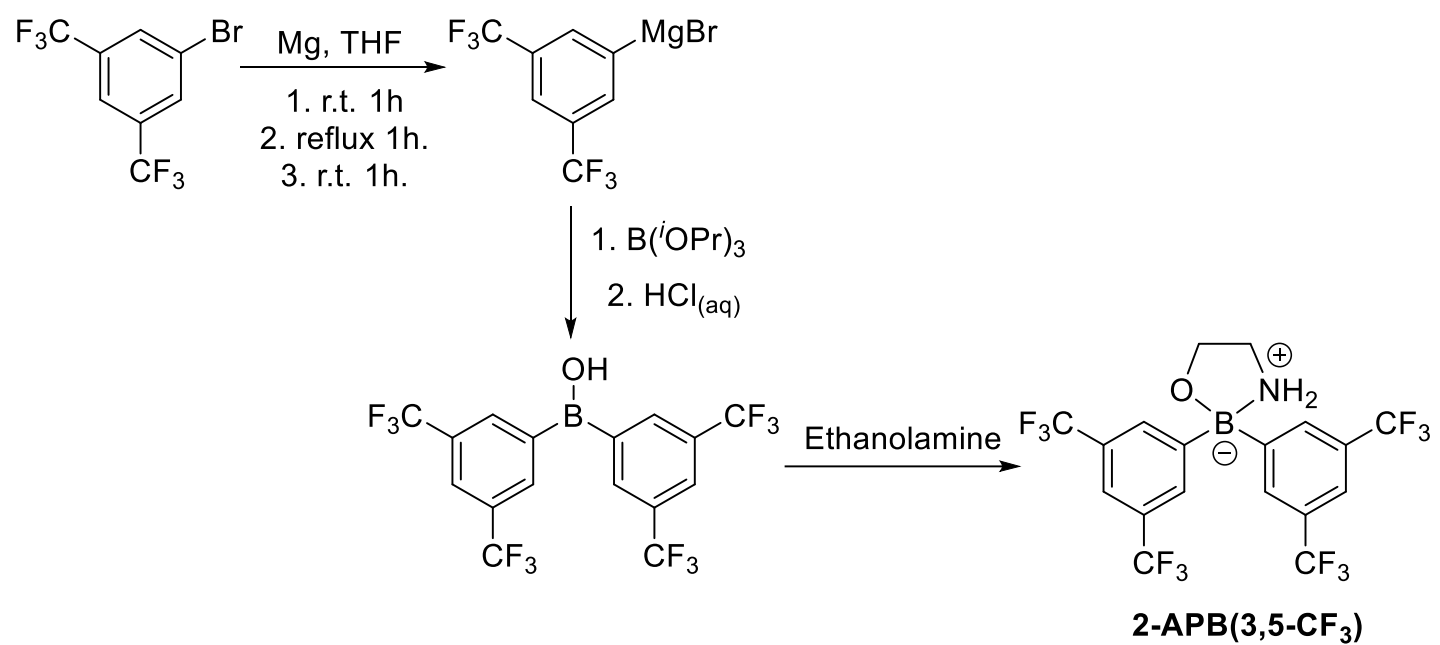

This unknown compound was synthesised according to the modified published procedures of synthesising 2-ABP class molecules. ${ }^{5}$ Under $\mathrm{N}_{2}$ atmosphere, THF (180 $\mathrm{mL})$ and magnesium turnings $(2.62 \mathrm{~g}, 0.108 \mathrm{~mol})$ were put into a well-dried roundbottom flask equipped with a condenser. 1,2-dibromoethane $(0.8 \mathrm{~g}, 4.26 \mathrm{mmol})$ was added to activate the magnesium. The mixture was stirred under reflux for at least 30 min. Then 3,5-bis(trifluoromethyl)bromobenzene $(15.5 \mathrm{~mL}, 89.9 \mathrm{mmol})$ was added into the flask dropwise at r.t. over $1 \mathrm{~h}$. After addition, the mixture was refluxed for $1 \mathrm{~h}$, then stirred at r.t. for another $1 \mathrm{~h}$. The resultant Grignard solution was dropwise cannula transferred into the THF $(100 \mathrm{~mL})$ solution of triisopropyl borate $(10 \mathrm{~mL}, 43.3 \mathrm{mmol})$ at $0{ }^{\circ} \mathrm{C}$. The mixture was then allowed to return to r.t. and stirred overnight. $1 \mathrm{M}$ hydrochloric acid was added to quench any unreacted Grignard reagent. The product 
was extracted using EtOAc and the combined organic phase was dried over $\mathrm{MgSO}_{4}$. The solution was then concentrated under reduced pressure and re-dissolved into 3:1 $\mathrm{EtOH} / \mathrm{H}_{2} \mathrm{O}(30 \mathrm{~mL})$. Ethanolamine $(4.5 \mathrm{~mL}, 74.6 \mathrm{mmol})$ was slowly added at $0{ }^{\circ} \mathrm{C}$. The mixture was then stirred at r.t. overnight. All precipitated solid was re-dissolved using EtOAc, and the crude product was washed by distilled water. The organic phase was combined and dried over $\mathrm{MgSO}_{4}$. All solvent was removed, and the product was purified by dissolving in $\mathrm{Et}_{2} \mathrm{O}$ followed by precipitation into chloroform. The product was obtained as pale orange crystals. Yield: $10.5 \mathrm{~g}, 48.8 \%$. ${ }^{1} \mathrm{H}$ NMR $(500 \mathrm{MHz}$, DMSO-d $)_{6} \delta 8.03(\mathrm{~s}, 4 \mathrm{H}), 7.77(\mathrm{~s}, 2 \mathrm{H}), 6.59(\mathrm{~m}, \mathrm{br}, 2 \mathrm{H}), 3.84(\mathrm{t}, J=6.5 \mathrm{~Hz}, 2 \mathrm{H}), 2.93$ $(\mathrm{p}, J=6.3 \mathrm{~Hz}, 2 \mathrm{H}) ;{ }^{19} \mathrm{~F}$ NMR (471 MHz, DMSO- $\left.d_{6}\right) \delta-61.2 ;{ }^{11} \mathrm{~B}$ NMR (160 MHz, DMSO- $\left.d_{6}\right) \delta 3.4$.

\subsubsection{Synthesis of StBPh2}

\subsubsection{Synthesis of $\mathrm{StBPh} \cdot \mathrm{NH}_{3}$}

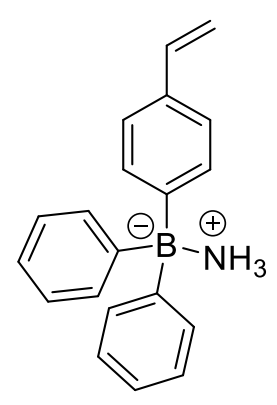

$\mathrm{StBPh}_{2} \cdot \mathrm{NH}_{3}$

Under $\mathrm{N}_{2}$ atmosphere with stirring, 1,2-dibromoethane was added dropwise into a mixture of anhydrous THF (54 mL) and magnesium turnings $(0.857 \mathrm{~g}, 0.0353 \mathrm{~mol})$. The mixture was refluxed for $30 \mathrm{~min}$. 4-Chlorostyrene (3.26 g, $23.5 \mathrm{mmol}$ ) was then added dropwise over $45 \mathrm{~min}$. The mixture was refluxed for an additional $30 \mathrm{~min}$, then Grignard reagent StMgCl was cannula transferred into THF solution of 2-APB (3.00 $\mathrm{g}, 11.8 \mathrm{mmol}$ ) dropwise at $-78^{\circ} \mathrm{C}$. The mixture was stirred at this temperature for $1 \mathrm{~h}$, then at $0{ }^{\circ} \mathrm{C}$ for $1 \mathrm{~h}$, and finally at r.t. for another $2 \mathrm{~h}$. Saturated $\mathrm{NH}_{4} \mathrm{Cl}$ aqueous solution $(50 \mathrm{~mL})$ was added and the mixture was stirred for $30 \mathrm{~min}$. The organic phase was collected, and the aqueous phase was extracted by diethyl ether $(20 \mathrm{~mL} \times 3)$. All organic phase was combined and dried over $\mathrm{MgSO}_{4}$. The solution was concentrated 
under reduced pressure. The resultant sticky oil was poured into hexane and the product precipitated as a white powder; Yield: $2.39 \mathrm{~g}, 74.2 \%$; ${ }^{1} \mathrm{H}$ NMR (500 MHz, DMSO- $\left.d_{6}\right) \delta 7.22-6.96(\mathrm{~m}, 14 \mathrm{H}), 6.64(\mathrm{dd}, J=17.6,10.9 \mathrm{~Hz}, 1 \mathrm{H}), 5.68(\mathrm{dd}, J=17.7$, $1.4 \mathrm{~Hz}, 1 \mathrm{H}), 5.59$ (s, br, 3H), 5.09 (dd, $J=10.9,1.4 \mathrm{~Hz}, 1 \mathrm{H}) ;{ }^{13} \mathrm{C}$ NMR $(126 \mathrm{MHz}$ DMSO-d6) $\delta 156.2$ (br), 155.4 (br), 137.6, 133.4, 133.2, 132.9, 126.2, 124.2, 124.0, 111.5; ${ }^{11} \mathrm{~B}$ NMR (161 MHz, DMSO- $\left.d_{6}\right) \delta$-4.9; EI-MS, $m / z,\left(\mathrm{M}^{+}\right)$calcd. for $\mathrm{C}_{20} \mathrm{H}_{20} \mathrm{BN}$ : 285.2, $\left(\left[\mathrm{M}-\mathrm{NH}_{3}\right]^{+}\right)$calcd. for $\mathrm{C}_{20} \mathrm{H}_{17} \mathrm{~B}: 268.2$. Found: 268.1 .

\subsubsection{Synthesis of StBPh2}<smiles>C=Cc1ccc(B(c2ccccc2)c2ccccc2)cc1</smiles>

4-Styryl-diphenylborane ammoniate (4.03 g, $14.1 \mathrm{mmol})$ was mixed with dry diethyl ether $(20 \mathrm{~mL})$ in a Schlenk flask. $\mathrm{HCl}$ etherate $(2 \mathrm{~N}$, $20 \mathrm{~mL}$ ) was transferred into the flask and the mixture was stirred at r.t. for $30 \mathrm{~min}$. The resultant white solid precipitate was removed by cannula filtration. Solvent and excess $\mathrm{HCl}$ was removed under vacuum. The crude product was purified by repeated recrystallisation from anhydrous $\mathrm{Et}_{2} \mathrm{O}$ until no hydrolysed product could be observed by NMR spectroscopy. The product, 4-styryl-diphenylborane, was obtained as a white powder; Yield: 2.25 g, 59.4 \%; m.p. 90-95 ${ }^{\circ} \mathrm{C}$; ${ }^{1} \mathrm{H}$ NMR (500 MHz, $\left.\mathrm{CDCl}_{3}\right), \delta 7.77-$ $7.67(\mathrm{~m}, 6 \mathrm{H}), 7.67-7.60(\mathrm{~m}, 2 \mathrm{H}), 7.60-7.56(\mathrm{~m}, 2 \mathrm{H}), 7.53(\mathrm{t}, J=7.5 \mathrm{~Hz}, 4 \mathrm{H}), 6.88(\mathrm{dd}$, $J=17.6,10.9 \mathrm{~Hz}, 1 \mathrm{H}), 5.97(\mathrm{dd}, J=17.7,0.9 \mathrm{~Hz}, 1 \mathrm{H}), 5.43(\mathrm{~d}, J=10.9,0.9 \mathrm{~Hz}, 1 \mathrm{H})$; ${ }^{13} \mathrm{C}$ NMR (126 MHz, $\mathrm{CDCl}_{3}$ ), $\delta 143.3$ (br), 142.9 (br), 140.4, 139.2, 138.7, 137.0, 131.4, 127.5, 125.4, 115.6; ${ }^{11} \mathrm{~B} \mathrm{NMR}\left(161 \mathrm{MHz}, \mathrm{CDCl}_{3}\right) \delta 67.2$. 


\subsubsection{Attempted synthesis of $\mathrm{StB}\left(4-\mathrm{CF}_{3}-\mathrm{Ph}\right)_{2}$}
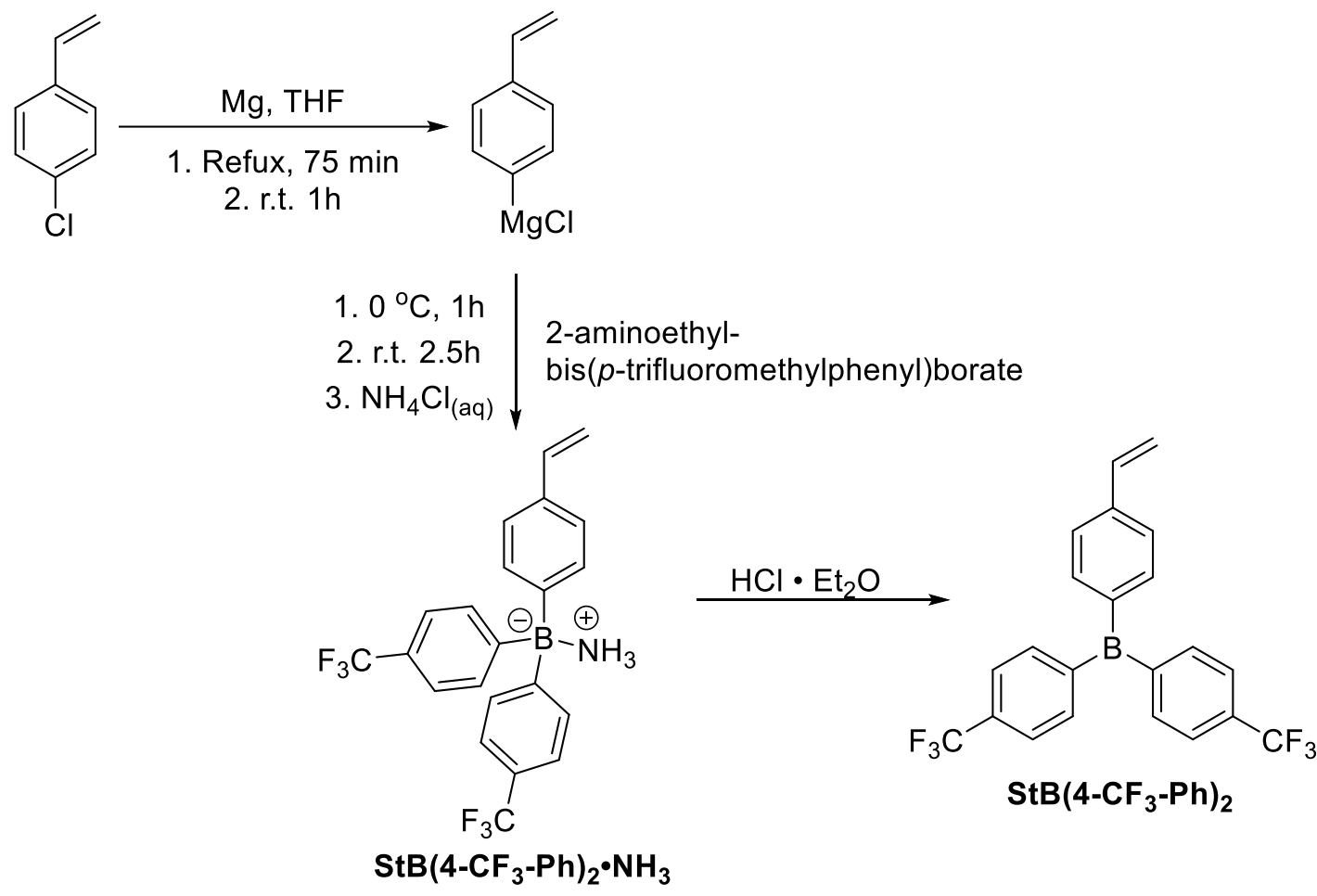

The Grignard reagent $\mathbf{S t M g C l}$ was prepared using similar approach stated before. The reagents used include 1,2-dibromoethane $(0.4 \mathrm{~mL}, 4.64 \mathrm{mmol})$, magnesium (1.91 g, $78.6 \mathrm{mmol})$, 4-chlorostyrene $(7.87 \mathrm{~mL}, 65.6 \mathrm{mmol})$ and THF $(100 \mathrm{~mL})$. Then the Grignard solution was dropwise cannula transferred into the THF $(62 \mathrm{~mL})$ solution of 2-APB(4-CF $)(9.47 \mathrm{~g}, 26.2 \mathrm{mmol})$ at $0{ }^{\circ} \mathrm{C}$ over $1 \mathrm{~h}$, then the mixture was allowed to return to r.t. and stirred for another $2.5 \mathrm{~h}$. Saturated $\mathrm{NH}_{4} \mathrm{Cl}$ aqueous solution was added to quench the reaction. The product was extracted using diethyl ether $(50 \mathrm{~mL} \times 3)$ and the combined organic phase was dried over $\mathrm{MgSO}_{4}$. The solution was concentrated under reduced pressure and then dropwise poured into hexane. The ammoniate product was precipitated as a white powder. Yield: $6.37 \mathrm{~g}, 57.7 \%$. ${ }^{1} \mathrm{H}$ NMR (500 MHz, DMSOd6) $\delta 12.22(\mathrm{~d}, J=7.6 \mathrm{~Hz}, 4 \mathrm{H}), 12.09(\mathrm{~d}, J=7.5 \mathrm{~Hz}, 4 \mathrm{H}), 12.00(\mathrm{~d}, J=7.9 \mathrm{~Hz}, 2 \mathrm{H})$, $11.90(\mathrm{~d}, J=8.0 \mathrm{~Hz}, 2 \mathrm{H}), 11.41(\mathrm{dd}, J=17.6,10.9 \mathrm{~Hz}, 1 \mathrm{H}), 10.62$ (s, 3H), 10.46 (dd, $J=17.7,1.3 \mathrm{~Hz}, 1 \mathrm{H}), 9.88(\mathrm{dd}, J=10.9,1.3 \mathrm{~Hz}, 1 \mathrm{H}) ;{ }^{19} \mathrm{~F}$ NMR $(471 \mathrm{MHz}$, DMSO$\left.d_{6}\right) \delta-60.4 ;{ }^{11} \mathrm{~B}$ NMR $\left(160 \mathrm{MHz}, \mathrm{DMSO}-d_{6}\right) \delta-5.1$. 
The ammoniate product ( $3 \mathrm{~g}, 7.12 \mathrm{mmol})$ was put into anhydrous diethyl ether (50 $\mathrm{mL})$, and then $\mathrm{HCl}$ etherate solution $(2 \mathrm{~N}, 50 \mathrm{~mL})$ was introduced into the mixture. After stirring at r.t. for $30 \mathrm{~min}$, any solid was filtered off, and the product was attempted to be recrystallised from diethyl ether and hexane. However, only a pale-yellow sticky oil can be obtained at this stage.

\subsubsection{Attempted synthesis of $\mathrm{StB}\left(2-\mathrm{CF}_{3}-\mathrm{Ph}\right)_{2} \cdot \mathrm{NH}_{3}$}

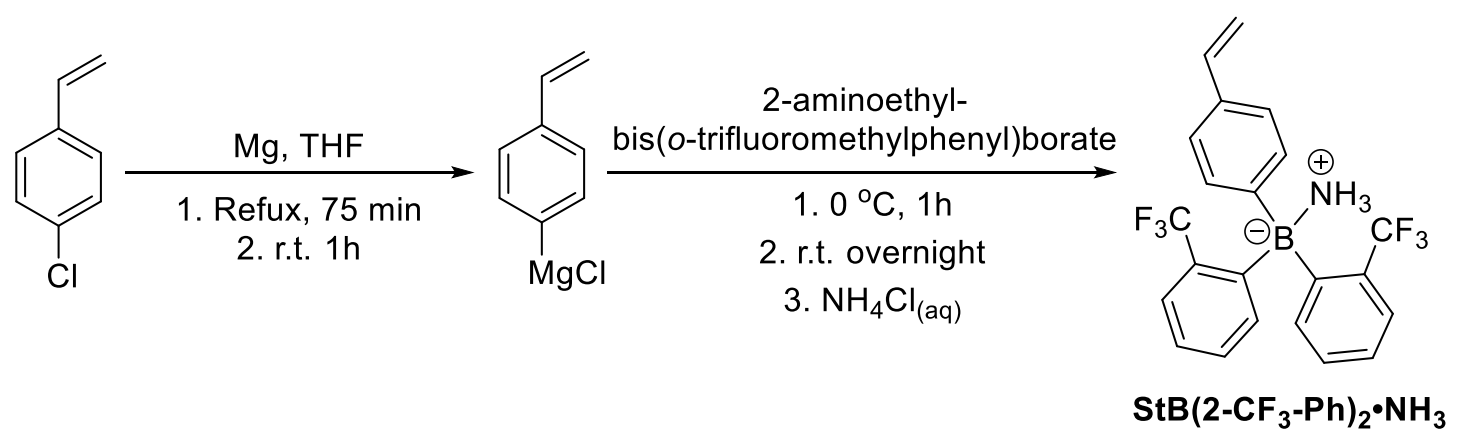

The Grignard reagent $\mathbf{S t M g C l}$ was prepared using similar approach stated in previous sections. The reagents used include 1,2-dibromoethane $(0.08 \mathrm{~mL}, 0.928 \mathrm{mmol})$, magnesium (0.299 g, $12.3 \mathrm{mmol})$, 4-chlorostyrene (1.23 mL, $10.3 \mathrm{mmol})$ and THF (20 $\mathrm{mL}$ ). Then the Grignard solution was dropwise cannula transferred into the THF (14 $\mathrm{mL})$ solution of $\mathbf{2 - A P B}\left(\mathbf{2}-\mathbf{C F}_{3}\right)(1.49 \mathrm{~g}, 4.12 \mathrm{mmol})$ at $0{ }^{\circ} \mathrm{C}$ over $1 \mathrm{~h}$, then the mixture was allowed to return to r.t. and stirred overnight. Saturated $\mathrm{NH}_{4} \mathrm{Cl}$ aqueous solution was added to quench the reaction. The product was extracted using diethyl ether (50 $\mathrm{mL} \times 3)$ and the combined organic phase was dried over $\mathrm{MgSO}_{4}$. The solution was concentrated under reduced pressure and then dropwise poured into hexane. No pure ammoniate product can be obtained. 


\subsubsection{Attempted synthesis of $\mathrm{StB}\left(3,5-\mathrm{CF}_{3}-\mathrm{Ph}\right)_{2} \cdot \mathrm{NH}_{3}$}

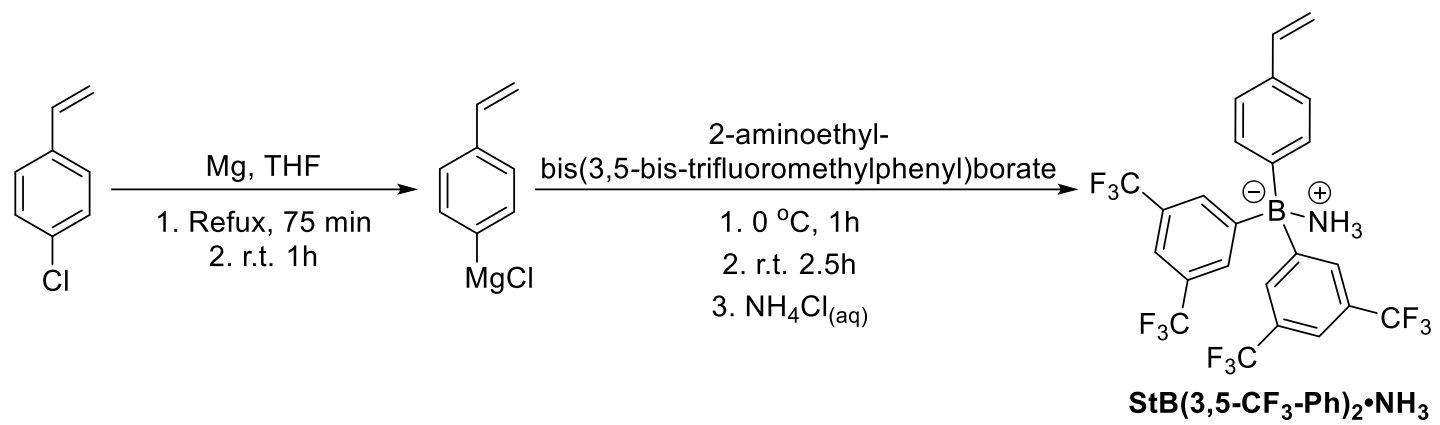

The Grignard reagent StMgCl was prepared using similar approach stated in previous sections. The reagents used include 1,2-dibromoethane $(0.12 \mathrm{~mL}, 1.39 \mathrm{mmol})$, magnesium (0.610 g, $25.1 \mathrm{mmol})$, 4-chlorostyrene (2.51 mL, $20.9 \mathrm{mmol})$ and THF (33 $\mathrm{mL}$ ). Then the Grignard solution was dropwise cannula transferred into the THF (20 $\mathrm{mL})$ solution of $\mathbf{2}-\mathbf{A P B}\left(\mathbf{3}, \mathbf{5}-\mathbf{C F}_{3}\right)(4.16 \mathrm{~g}, 8.37 \mathrm{mmol})$ at $0{ }^{\circ} \mathrm{C}$ over $1 \mathrm{~h}$, then the mixture was allowed to return to r.t. and stirred for another $2.5 \mathrm{~h}$. Saturated $\mathrm{NH}_{4} \mathrm{Cl}$ aqueous solution was added to quench the reaction. The product was extracted using diethyl ether $(50 \mathrm{~mL} \times 3)$ and the combined organic phase was dried over $\mathrm{MgSO}_{4}$. The solution was concentrated under reduced pressure and then dropwise poured into hexane. No collectable product could be obtained.

\subsubsection{Synthesis of $\left(\mathrm{StF}_{4}\right) \mathrm{B}\left(\mathrm{C}_{6} \mathrm{~F}_{5}\right)_{2}$}

\subsubsection{Synthesis of 2,3,5,6-tetrafluoro-4-bromobenzaldehyde}

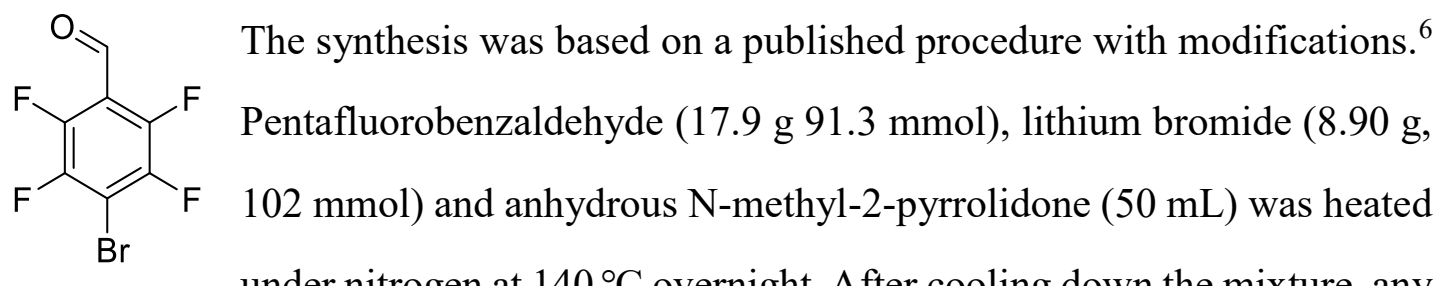
under nitrogen at $140{ }^{\circ} \mathrm{C}$ overnight. After cooling down the mixture, any solid precipitated was filtered off through a celite plug, and the solution obtained was slowly poured into distilled water to precipitate out the product. The product was then 
collected by filtration. The filtrate was collected and extracted by diethyl ether. The combined organic phase was dried over $\mathrm{MgSO}_{4}$. The mixture was concentrated, then poured into distilled water again to collect the second portion of the product. All product obtained was dried over phosphorous pentoxide under vacuum for 3 days. The product was obtained as a pale yellow solid. Yield: 19.6 g, 83.5\%. ${ }^{1} \mathrm{H}$ NMR (400 MHz, $\left.\mathrm{CDCl}_{3}\right) \delta 10.30(\mathrm{p}, J=1.1 \mathrm{~Hz}, 1 \mathrm{H}) ;{ }^{13} \mathrm{C} \mathrm{NMR}\left(101 \mathrm{MHz}, \mathrm{CDCl}_{3}\right) \delta 182.0,147.2,144.5$, 114.6, 107.5; ${ }^{19} \mathrm{~F}$ NMR (376 MHz, $\left.\mathrm{CDCl}_{3}\right) \delta-131.2(\mathrm{~m}),-144.0(\mathrm{~m})$.

\subsubsection{Synthesis of $\mathrm{StF}_{4} \mathrm{Br}$}<smiles>C=Cc1c(F)c(F)c(Br)c(F)c1F</smiles>

$\mathrm{StF}_{4} \mathrm{Br}$

2,3,5,6-tetrafluoro-4-bromobenzaldehyde (5.00 $\mathrm{g}, 19.5 \mathrm{mmol})$ was dissolved in $\mathrm{Et}_{2} \mathrm{O}(50 \mathrm{~mL})$ and the solution was cooled to $0{ }^{\circ} \mathrm{C}$. Methylmagnesium bromide etherate solution (3M, 6.15 mL, $18.5 \mathrm{mmol})$ was added dropwise at this temperature over $1 \mathrm{~h}$, then the mixture was stirred at r.t. overnight. Saturated $\mathrm{NH}_{4} \mathrm{Cl}$ aqueous solution was added to quench the reaction. The product was extracted by diethyl ether $(50 \mathrm{~mL} \times 3)$, and the combined organic phase was dried over $\mathrm{MgSO}_{4}$. After removal of solvent, the product 1-(2,3,5,6-tetrafluoro-4-bromophenyl)ethanol was purified by column chromatography (100/0-90/10 hex/EtOAc) to give a pale yellow oil. Yield: $3.67 \mathrm{~g}, 13.4$ mmol. ${ }^{1} \mathrm{H}$ NMR (400 MHz, $\left.\mathrm{CDCl}_{3}\right) \delta 5.25(\mathrm{q}, J=6.7 \mathrm{~Hz}, 1 \mathrm{H}), 2.71(\mathrm{~s}, 1 \mathrm{H}), 1.62(\mathrm{~d}, J$ $=6.8 \mathrm{~Hz}, 3 \mathrm{H}) ;{ }^{13} \mathrm{C} \mathrm{NMR}\left(101 \mathrm{MHz}, \mathrm{CDCl}_{3}\right) \delta 145.1(\mathrm{ddm}, J=248.116 .2 \mathrm{~Hz}), 144.8$ $(\mathrm{dm}, J=250.2 \mathrm{~Hz}), 122.72(\mathrm{t}, J=15.1 \mathrm{~Hz}), 99.0(\mathrm{tt}, J=22.7,2.2 \mathrm{~Hz}), 62.66$ (p, $J=$ $2.3 \mathrm{~Hz}), 22.99(\mathrm{t}, J=1.6 \mathrm{~Hz}) ;{ }^{19} \mathrm{~F}$ NMR $\left(376 \mathrm{MHz}, \mathrm{CDCl}_{3}\right) \delta-133.5(\mathrm{~m}),-143.5(\mathrm{~m})$.

The product obtained above ( $40 \mathrm{~g}, 0.147 \mathrm{~mol})$ was mixed with hydroquinone $(2 \mathrm{~g}, 18.2$ mmol) and $\mathrm{H}_{3} \mathrm{PO}_{4}(85 \%, 84.5 \mathrm{~g})$. The mixture was heated at $140{ }^{\circ} \mathrm{C}$ for $4 \mathrm{~h}$ with a condenser. After cooling down, the product was extracted using diethyl ether and the combined organic phase was dried over $\mathrm{MgSO}_{4}$. After removal of solvent, the product 
was purified by flushing though a silica plug using pentane. The product was obtained as a colorless oil. Yield: $22.0 \mathrm{~g}, 58.9 \%$. ${ }^{1} \mathrm{H}$ NMR $\left(400 \mathrm{MHz}, \mathrm{CDCl}_{3}\right) \delta 6.66(\mathrm{dd}, J=$ 18.0, $11.9 \mathrm{~Hz}, 1 \mathrm{H}), 6.13(\mathrm{dd}, J=18.0,0.8 \mathrm{~Hz}, 1 \mathrm{H}), 5.76(\mathrm{dd}, J=12.0,0.7 \mathrm{~Hz}, 1 \mathrm{H})$; ${ }^{13} \mathrm{C}$ NMR $\left(101 \mathrm{MHz}, \mathrm{CDCl}_{3}\right) \delta 145.2(\mathrm{dm}, J=247.0 \mathrm{~Hz}), 145.0(\mathrm{t}, J=248.0 \mathrm{~Hz})$, $124.4(\mathrm{t}, J=7.9 \mathrm{~Hz}), 122.20(\mathrm{p}, J=2.4 \mathrm{~Hz}), 116.50(\mathrm{t}, J=13.6 \mathrm{~Hz}), 98.4(\mathrm{tt}, J=22.8$, $2.0 \mathrm{~Hz}) ;{ }^{19} \mathrm{~F}$ NMR $\left(376 \mathrm{MHz}, \mathrm{CDCl}_{3}\right) \delta-134.7(\mathrm{~m}),-142.4(\mathrm{~m})$.

\subsubsection{Synthesis of $\left(\mathrm{StF}_{4}\right) \mathrm{B}\left(\mathrm{C}_{6} \mathrm{~F}_{5}\right)_{2}$}<smiles>C=Cc1c(F)c(F)c(B(c2c(F)c(F)c(F)c(F)c2F)c2c(F)c(F)c(F)c(F)c2F)c(F)c1F</smiles>

$\left(\mathrm{StF}_{4}\right) \mathrm{B}\left(\mathrm{C}_{6} \mathrm{~F}_{5}\right)_{2}$

Under nitrogen, magnesium turnings (2.29 g, $94.2 \mathrm{mmol})$ was put into anhydrous diethyl ether $(80.0 \mathrm{~mL})$ in a sealed round bottom flask. A crystal of iodine was added to activate the magnesium. Then the mixture was cooled to $0{ }^{\circ} \mathrm{C}$ and pentafluorobromobenzene (19.4 g, $78.5 \mathrm{mmol})$ was added dropwise over $1 \mathrm{~h}$. Then the mixture was stirred at this temperature for another $1 \mathrm{~h}$. The Grignard formed was dropwise cannula transferred into $\mathrm{Et}_{2} \mathrm{O}(66.0 \mathrm{~mL})$ solution of $\mathrm{BF}_{3}$ etherate $(4.95 \mathrm{~mL}, 39.2 \mathrm{mmol})$ at $0{ }^{\circ} \mathrm{C}$. The mixture was stirred at this temperature for another $3 \mathrm{~h}$ to give a crude solution of bis(pentafluorophenyl)boron fluoride.

Under nitrogen, 2,3,5,6-tetrafluoro-4-bromostyrene (10.0 g, $39.2 \mathrm{mmol})$ in $\mathrm{Et}_{2} \mathrm{O}$ (120 $\mathrm{mL}$ ) was dropwise added into the mixture of diethyl ether $(41.0 \mathrm{~mL})$ and activated magnesium turnings $(2.86 \mathrm{~g}, 118 \mathrm{mmol})$ at $0{ }^{\circ} \mathrm{C}$ over $3 \mathrm{~h}$. After that, the mixture was further stirred at this temperature for $1 \mathrm{~h}$. Then the Grignard reagent formed was dropwise cannula transferred into the prepared bis(pentafluorophenyl)boron fluoride solution at $0{ }^{\circ} \mathrm{C}$. The mixture was then allowed to return to r.t. and stirred overnight. All solvent was removed under the reduced temperature at low temperature, then hexane $(150 \mathrm{~mL} \times 3)$ was added to extract the product at $45^{\circ} \mathrm{C}$. The solution obtained was combined and the solvent was completely removed under vacuum. The solid crude 
product was purified by repeated recrystallisation from hexane. The desired product 4-tetrafluorostyryl-bis(pentafluorophenyl)borane was obtained as white needles. Yield: $9.5 \mathrm{~g}, 46.6 \%$. ${ }^{1} \mathrm{H}$ NMR (400 MHz, toluene- $\left.d_{8}\right) \delta 6.33(\mathrm{dd}, J=18.0,11.9 \mathrm{~Hz}, 1 \mathrm{H}), 5.90$ $(\mathrm{d}, J=17.9 \mathrm{~Hz}, 1 \mathrm{H}), 5.32(\mathrm{~d}, J=11.9 \mathrm{~Hz}, 1 \mathrm{H}) ;{ }^{19} \mathrm{~F}$ NMR $\left(376 \mathrm{MHz}\right.$, toluene- $\left.d_{8}\right) \delta-$ $128.5(\mathrm{~m}),-130.1(\mathrm{~m}),-142.1(\mathrm{dm}, J=281.4 \mathrm{~Hz}),-142.7(\mathrm{~m}),-160.0(\mathrm{dm}, J=105.8$ $\mathrm{Hz}) ;{ }^{11} \mathrm{~B}$ NMR $\left(128 \mathrm{MHz}\right.$, toluene- $\left.d_{8}\right) \delta 59.9$.

\subsection{Determination of Lewis Acidity}

\subsubsection{General procedure for the determination of Lewis acidities by Gutmann-Beckett Method.}

NMR analytical amount of a Lewis acid-containing monomer/polymer was dissolved in a certain anhydrous deuterated solvent. One equivalent of triethylphosphine oxide was added and the mixture was stirred overnight. Then the ${ }^{31} \mathrm{P}$ NMR spectrum was tested and the acceptor number was calculated.

\subsubsection{Acceptor Number of StBPh 2 in $\mathrm{CD}_{2} \mathrm{Cl}_{2}$}
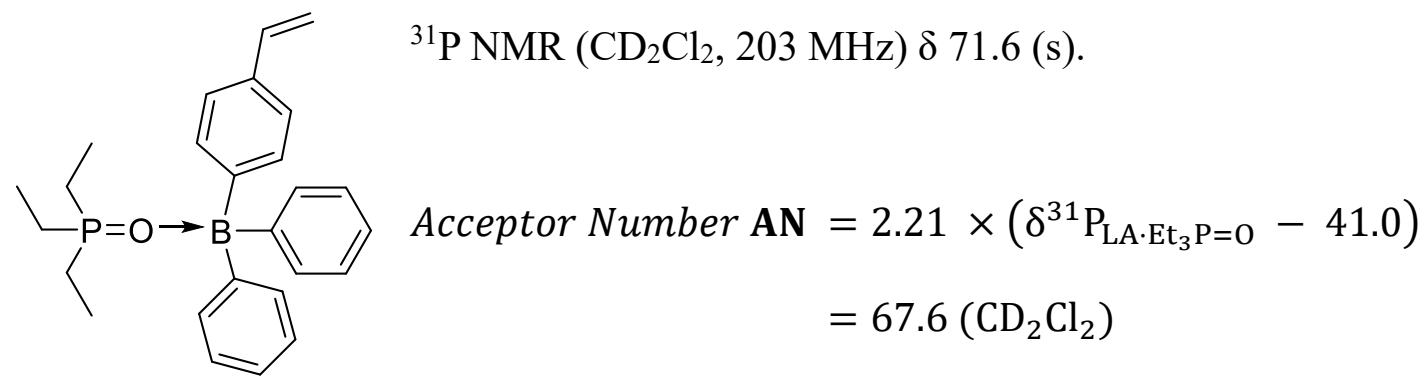


\subsubsection{Acceptor Number of Copolymer of Styrene and StBPh2}

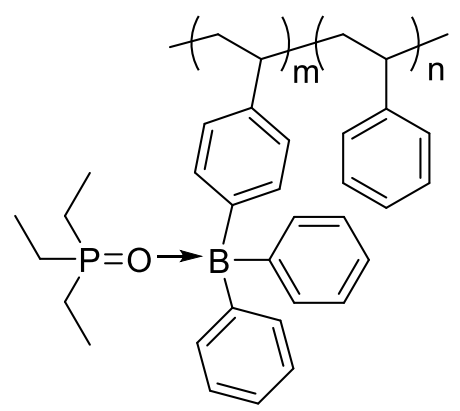

$$
\begin{aligned}
& { }^{31} \mathrm{P} \text { NMR }\left(\mathrm{CD}_{2} \mathrm{Cl}_{2}, 203 \mathrm{MHz}\right) \delta 64.5(\mathrm{~s}, \mathrm{br}) . \\
& \text { Acceptor Number } \mathbf{A N}=2.21 \times\left(\delta^{31} \mathrm{P}_{\mathrm{LA} \cdot \mathrm{Et}_{3} \mathrm{P}=\mathrm{O}}-41.0\right) \\
& =51.9\left(\mathrm{CD}_{2} \mathrm{Cl}_{2}\right) \\
& \left.{ }^{31} \mathrm{P} \text { NMR (toluene- } d_{8}, 202.5 \mathrm{MHz}\right) \delta 67.5(\mathrm{~s}, \mathrm{br}) \\
& \text { Acceptor Number } \mathbf{A N}=2.21 \times\left(\delta^{31} \mathrm{P}_{\mathrm{LA} \cdot \mathrm{Et}_{3} \mathrm{P}=\mathrm{O}}-41.0\right) \\
& =58.5\left(\text { Toluene }-d_{8}\right)
\end{aligned}
$$

\subsubsection{Acceptor Number of $\left(\mathrm{StF}_{4}\right) \mathrm{B}_{(}\left(\mathrm{C}_{6} \mathrm{~F}_{5}\right)_{2}$}

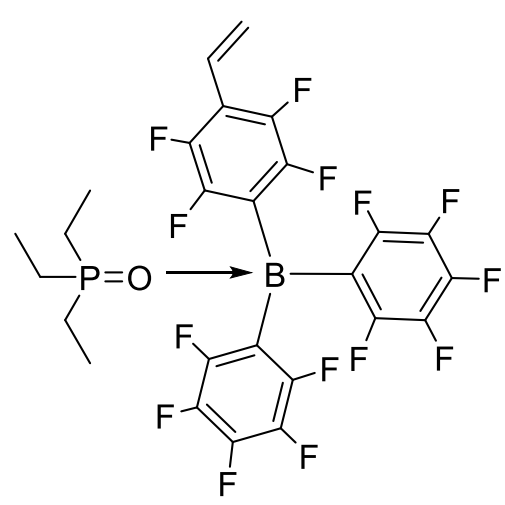

${ }^{31} \mathrm{P}$ NMR (Toluene- $\left.d_{8}, 203 \mathrm{MHz}\right) \delta 75.0(\mathrm{~d}, J=50.4$ $\mathrm{Hz})$.

Acceptor Number AN

$$
\begin{aligned}
& =2.21 \times\left(\delta^{31} \mathrm{P}_{\mathrm{LA} \cdot \mathrm{Et}} \mathrm{P}=\mathrm{O}-41.0\right) \\
& =75.1\left(\text { Toluene }-d_{8}\right)
\end{aligned}
$$

\subsection{Determination of Lewis Basicity}

\subsubsection{General procedure for the determination of Lewis basicity by preparation of phosphine selenide compounds.}

The phosphine selenide compounds were prepared by modified published procedures. ${ }^{7}$ Either pure or crude phosphine-containing monomer was mixed with five equivalent of selenium pellets or powder in degassed chloroform or toluene. The mixture was stirred at $70{ }^{\circ} \mathrm{C}$ overnight. Then the solution was filtered, concentrated and purified by column chromatography/recrystallisation to give the desired phosphine selenide compound. The resultant product was characterised by ${ }^{31} \mathrm{P} \mathrm{NMR}$ in $\mathrm{CDCl}_{3}$ to work- 
out the ${ }^{1} J_{\text {PSe value. }}$

\subsubsection{Synthesis of $\mathrm{StP}(=\mathrm{Se}) \mathrm{Ph}_{2}$}<smiles>C=Cc1ccc(P(=O)(c2ccccc2)c2ccccc2)cc1</smiles>

StPPh2 (1.00 g, $3.47 \mathrm{mmol}$ ) was dissolved in degassed chloroform (87.0 $\mathrm{mL})$, and selenium pellets $(1.37 \mathrm{~g}, 17.4 \mathrm{mmol})$ was added. The mixture was stirred under $\mathrm{N}_{2}$ at $70{ }^{\circ} \mathrm{C}$ overnight. After that, the mixture was cooled down and unreacted selenium was filtered off. The solution was concentrated and purified by column chromatography $\left(\mathrm{CHCl}_{3}\right)$ $\mathbf{S t P}(=\mathbf{S e}) \mathrm{Ph}_{2}$ using $\mathrm{Al}_{2} \mathrm{O}_{3}$. The product can also be recrystallised by dissolving into THF and followed by precipitation into methanol. The product was obtained as a white/pale red solid. Yield: $1.04 \mathrm{~g}, 81.6 \%$; ${ }^{1} \mathrm{H}$ NMR (400 MHz, $\mathrm{CDCl}_{3}$ ), $\delta 7.85$ - 7.37 $(\mathrm{m}, 14 \mathrm{H}), 6.76(\mathrm{dd}, J=17.6,10.9 \mathrm{~Hz}, 1 \mathrm{H}), 5.87(\mathrm{~d}, J=17.6 \mathrm{~Hz}, 1 \mathrm{H}), 5.40(\mathrm{~d}, J=10.9$ $\mathrm{Hz}, 1 \mathrm{H}) ;{ }^{31} \mathrm{P}$ NMR $\left(162 \mathrm{MHz}, \mathrm{CDCl}_{3}\right), \delta 34.7\left({ }^{1} J_{\mathrm{PSe}}=729.3 \mathrm{~Hz}\right)$.

\subsubsection{Synthesis of StP(=Se)Mes2}

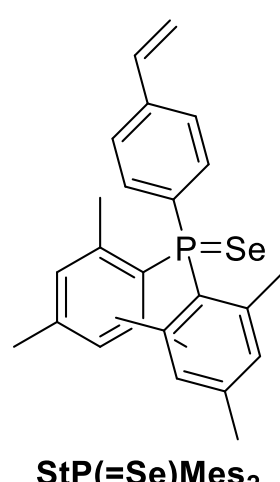

StPMes2 (1.00 g, $2.68 \mathrm{mmol})$ was dissolved in degassed chloroform, and selenium pellets (1.06 g, $13.4 \mathrm{mmol})$ was added. The mixture was stirred under $\mathrm{N}_{2}$ at $70{ }^{\circ} \mathrm{C}$ overnight. After that, the mixture was cooled down and unreacted selenium was filtered off. The solution was concentrated and purified by column chromatography $\left(\mathrm{CHCl}_{3} / \mathrm{Hexane} 20 / 80-40 / 60\right)$ using $\mathrm{Al}_{2} \mathrm{O}_{3}$. The product can also be recrystallised by dissolving into THF and followed by precipitation into methanol. The product was obtained as a pale yellow solid. Yield: $0.937 \mathrm{~g}, 77.4 \%$; ${ }^{1} \mathrm{H}$ NMR $\left(400 \mathrm{MHz}, \mathrm{CDCl}_{3}\right), \delta 8.15$ (s, br, 2H), $7.52-$ $7.34(\mathrm{~m}, 2 \mathrm{H}), 6.81(\mathrm{~d}, J=4.2 \mathrm{~Hz}, 4 \mathrm{H}), 6.74(\mathrm{dd}, J=17.6,10.9 \mathrm{~Hz}, 1 \mathrm{H}), 5.86(\mathrm{~d}, J=$ $17.6 \mathrm{~Hz}, 1 \mathrm{H}), 5.38$ (d, $J=10.9 \mathrm{~Hz}, 1 \mathrm{H}), 2.27$ (s, 6H), 2.18 (s, 12H); ${ }^{31} \mathrm{P}$ NMR (162 
$\left.\mathrm{MHz}, \mathrm{CDCl}_{3}\right), \delta 19.4\left({ }^{1} J_{\mathrm{PSe}}=695.4 \mathrm{~Hz}\right)$.

\subsection{RAFT Polymerisations of Monomers}

\subsubsection{RAFT copolymerization of StPMes2 and styrene}

In a glovebox, weighted amount of StPMes2, styrene and cumyl dithiobenzoate was mixed together. Toluene was either added or not, depends on the relative amount of less soluble phosphine monomer added. The mixture was transferred into a well-sealed ampoule, and then heated outside the glovebox at $110{ }^{\circ} \mathrm{C}$ for desired time. After polymerisation, the mixture was cooled down, and a small amount of aliquot was collected for NMR to calculate the conversion. The mixture was vacuumed down to remove any volatile compounds, then re-dissolved in small amount of THF (1-2 mL). The resultant polymer solution was slowly poured into hexane. The product poly(styrene-co-4-styryldimeistylphosphine) (P1/P2/P3) was obtained as a pale pink solid. ${ }^{1} \mathrm{H}$ NMR (500 MHz, Toluene- $d_{8}$ ), $\delta$ 7.40-6.10 (m, br), 2.57-1.83 (m, br), 1.831.21 (br); ${ }^{31} \mathrm{P}$ NMR (203 MHz, Toluene- $\left.d_{8}\right), \delta-22.7$ (br)

\subsubsection{RAFT copolymerization of StBPh 2 and styrene}

In a glovebox, weighted amount of StBPh2, styrene, cumyl dithiobenzoate and 1,3,5trisisopropyl-2,4,6-dioxane was mixed together. Toluene was either added or not, depends on the relative amount of less soluble borane monomer added. One aliquot was collected before polymerisation for NMR. The mixture was transferred to a sealed ampoule and heated outside the glovebox at $110{ }^{\circ} \mathrm{C}$ for desired time. After cooling down, the ampoule was transferred back to glovebox and another aliquot was collected for NMR to calculate the conversion. The mixture was dissolved in small amount of toluene, then poured into hexane. The product poly(styrene-co-4-styryldiphenylborane) 
(B1/B2/B3) was obtained as a pale pink solid. ${ }^{1} \mathrm{H}$ NMR (500 MHz, Toluene- $\left.d 8\right), \delta$ 7.90-6.30 (m, br), 2.70-1.40 (m, br); ${ }^{11} \mathrm{~B}$ NMR (161 MHz, Toluene- $\left.d_{8}\right) \delta 67.8$.

\subsection{Network Formation from Polymeric FLPs}

\subsubsection{Binding Test between PhPMes2, $\mathrm{BPh}_{3}$ and DEAD}

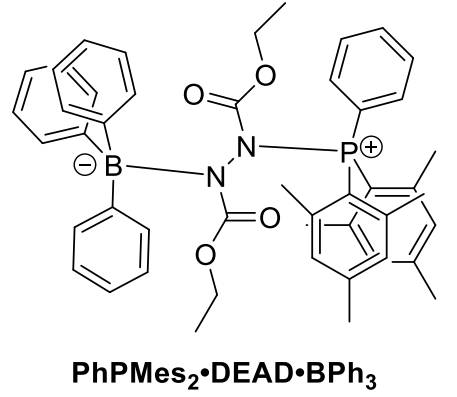

interval).

The binding test was performed using model compounds. BPh3 (0.028 g, 0.115 mmol), PhPMes2 (0.04 g, 0.115 mmol), and DEAD (0.0200 g, $0.115 \mathrm{mmol})$ was dissolved in deuterated toluene $(0.75 \mathrm{~mL})$. The solution was sealed in a Young's tap NMR tube and ${ }^{11} \mathrm{~B},{ }^{31} \mathrm{P}$ NMR spectra were recorded at varying temperatures $(300-370 \mathrm{~K}, 10 \mathrm{~K}$

\subsubsection{Gelation Experiments}

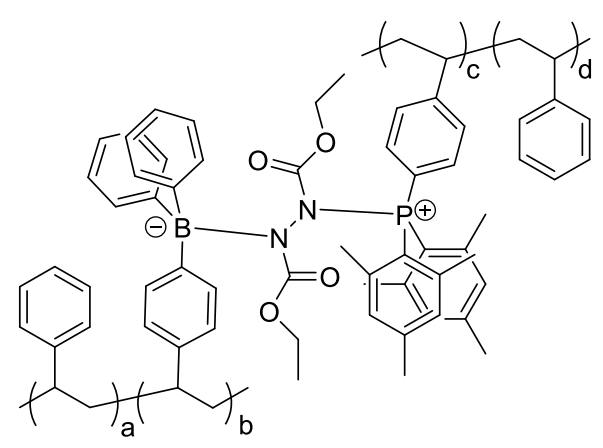

P1/P2/P3 and B1/B2/B3 were dissolved into anhydrous toluene with 1:1 equivalent of boron and phosphorous moieties. A stock toluene solution of DEAD (6 eqv.) was quickly injected into the solution. The total concentration of polymers in solution was kept at $4.50 \times 10^{-3}$ $\mathrm{mol} / \mathrm{dm}^{3}$ after the addition of DEAD solution. The mixture was allowed to stand at ambient temperature overnight. Then the upper clear solution was decanted off. The gel was washed with toluene three times before characterizations. 


\subsection{References}

[1] Seifert, A.; Mahrwald, R., Tetrahedron Lett. 2009, 50 (47), 6466-6468.

[2] Saindane, P.; Jagtap, R. N., Prog. Org. Coat. 2015, 79, 106-114.

[3] Campos, J.; Espada, M. F.; López-Serrano, J.; Carmona, E., Inorg. Chem. 2013, $52(11), 6694-6704$.

[4] Takeda, Y.; Nishida, T.; Minakata, S., Chem. Eur. J. 2014, 20 (33), 1026610270.

[5] Chen, X.; Ke, H.; Chen, Y.; Guan, C.; Zou, G., J. Org. Chem. 2012, 77 (17), $7572-7578$

[6] Martin, E.; Hughes, D. L.; Hursthouse, M. B.; Male, L.; Lancaster, S. J., Dalton Trans. 2009, (9), 1593-1601.

[7] Cinderella, A. P.; Vulovic, B.; Watson, D. A., J. Am. Chem. Soc. 2017, 139 (23), 7741-7744. 